Supporting Information for

\title{
A Three-Coordinate Copper(II) Alkynyl Complex in C-C Bond Formation: The Sesquicentennial of the Glaser Coupling
}

Abolghasem Bakhoda, ${ }^{1,2 \dagger}$ Otome E. Okoromoba, ${ }^{1,3 \dagger}$ Christine Greene, ${ }^{1}$ Mahdi Raghibi Boroujeni, ${ }^{1}$ Jeffery A. Bertke, ${ }^{1}$ and Timothy H. Warren ${ }^{1, *}$

${ }^{1}$ Department of Chemistry, Georgetown University, Box 571227, Washington, DC 20057-1227, United States

${ }^{2}$ Current Address: National Institute on Alcohol Abuse and Alcoholism, NIH, Bethesda, MD 20892, United States.

${ }^{3}$ Current Address: Department of Chemistry and Biochemistry, George Mason University, Fairfax, VA 22030, United States.

Corresponding author email: thw@georgetown.edu (T.H.W.)

\section{Table of Contents}

S1

1. General Instrumentation and Physical Methods $\quad$ S3

2. Materials $\quad$ S4

3. Synthesis and Characterization of $\left[{ }^{i} \mathrm{Pr}_{2} \mathrm{NN}\right] \mathrm{Cu}-\mathrm{O}^{t} \mathrm{Bu}(\mathbf{2}) \quad$ S5

4. Synthesis and Characterization of $\left[{ }^{i} \mathrm{Pr}_{2} \mathrm{NN}\right] \mathrm{Cu}-\mathrm{C} \equiv \mathrm{CAr}^{\mathrm{Cl} 2}(\mathbf{3}) \quad \mathbf{S 7}$

5. Observation of $\left[\mathrm{CoCp}_{2}\right]^{+}\left\{\left[{ }^{i} \mathrm{Pr}_{2} \mathrm{NN}\right] \mathrm{Cu}-\mathrm{C} \equiv \mathrm{CAr}^{\mathrm{Cl} 2}\right\}^{-}\left(\mathbf{C p}_{2} \mathbf{C o}^{+}-\mathbf{4}\right) \quad \mathbf{S 1 1}$

6. Thermal Decay of $\left[{ }^{i} \mathrm{Pr}_{2} \mathrm{NN}\right] \mathrm{Cu}-\mathrm{C} \equiv \mathrm{CAr}^{\mathrm{Cl} 2}$ (3) $\mathbf{S 1 2}$

7. Decay of $\left[{ }^{i} \mathrm{Pr}_{2} \mathrm{NN}\right] \mathrm{Cu}-\mathrm{C} \equiv \mathrm{CAr}^{\mathrm{Cl} 2}$ (3) Promoted by Acetonitrile and $\begin{array}{ll}2,4-\text { Lutidine } & \mathbf{S 1 4}\end{array}$

8. Reactivity of $\left[{ }^{i} \mathrm{Pr}_{2} \mathrm{NN}\right] \mathrm{Cu}-\mathrm{C} \equiv \mathrm{CAr}^{\mathrm{Cl} 2}$ (3) with Various Alkynide Anions $\quad$ S16

9. Reactivity of $\left[{ }^{i} \mathrm{Pr}_{2} \mathrm{NN}\right] \mathrm{Cu}-\mathrm{C} \equiv \mathrm{CAr}{ }^{\mathrm{Cl} 2}$ with Different Equivalents $\begin{array}{ll}\text { of (2,6-Dichlorophenyl)ethyn-1-ide } & \text { S24 }\end{array}$

10. Reactivity of $\left[{ }^{i} \mathrm{Pr}_{2} \mathrm{NN}\right] \mathrm{Cu}-\mathrm{C} \equiv \mathrm{CAr}^{\mathrm{Cl} 2}$ (3) with Phenyllithium $\quad$ S26

11. Trityl Radical Capture by $\left[{ }^{i} \mathrm{Pr}_{2} \mathrm{NN}\right] \mathrm{Cu}-\mathrm{C} \equiv \mathrm{CAr}^{\mathrm{Cl} 2}(\mathbf{3}) \quad \mathbf{S 2 8}$

12. Procedures for Catalytic Alkylation of Terminal Alkynes Catalyzed by $\left[\mathrm{Cl}_{2} \mathrm{NN}\right] \mathrm{Cu}$

13. Crystallographic Details and Additional Structures $\quad$ S44

14. References for Synthetic and Crystallographic Details $\quad$ S49

15. Computational Details $\quad$ S51

16. Spectral Data $\quad$ S108 


\section{General Instrumentation and Physical Methods.}

The preparation and handling of air-sensitive chemicals were done under dry nitrogen atmosphere by utilizing MBraun gloveboxes and/or standard Schlenk techniques unless otherwise mentioned. ${ }^{1} \mathrm{H},{ }^{13} \mathrm{C}\left\{{ }^{1} \mathrm{H}\right\}$, and ${ }^{19} \mathrm{~F}$ NMR spectra were recorded on a Varian $400 \mathrm{MHz}$ spectrometer at room temperature unless otherwise noted. The chemical shift $(\delta)$ values are expressed in ppm relative to tetramethylsilane, whereas the residual ${ }^{1} \mathrm{H}$ signal of deuterated solvent served as an internal standard. ${ }^{19} \mathrm{~F}$ NMR spectra were recorded in presence of an internal reference of fluorobenzene ( $\delta=-113.15 \mathrm{ppm}$ vs. $\left.\mathrm{CFCl}_{3} \delta=0.00\right)$. Elemental analyses were performed on a Perkin-Elmer PE2400 microanalyzer at Georgetown University. UV-vis spectra were recorded on Agilent 8454 Diode Array spectrometer equipped with stirrer and Unisoku USP-203 cryostat for variable temperature $\left(-105{ }^{\circ} \mathrm{C}\right.$ to $\left.90{ }^{\circ} \mathrm{C}\right)$ experiments. The molar extinction coefficients of different isolated complexes were determined from Beer's law plots (absorbance vs concentration) with at least four different concentrations. IR spectra (with spectral resolution of $2 \mathrm{~cm}^{-1}$ ) were collected on a Varian $3100 \mathrm{FT}-\mathrm{IR}$ spectrometer by using $\mathrm{KBr}$ pellet method unless otherwise mentioned. Cyclic voltammetry measurements were done at room temperature under dry nitrogen atmosphere of a glovebox using a BASi Epsilon Electrochemistry setup with three electrodes (Working electrode: glassy carbon, Auxiliary electrode: platinum wire, Pseudo-reference electrode: silver wire). GC-MS analyses of the reaction mixtures were performed on a Varian Saturn 2100T equipped with a Varian $\mathrm{CP}-8400$ auto sampler. Identification of products was carried out by comparing their retention times, electron ionization (EI) mass spectra, and NMR spectra with those of the authentic compounds. EPR spectra were collected on a JEOL continuous wave spectrometer JES-FA200 equipped with an X-band Gunn oscillator bridge, a cylindrical mode cavity, and a liquid nitrogen cryostat. EPR measurements were performed in sealed quartz tubes. All spectra were obtained on freshly prepared solutions in toluene (at least two independently synthesized batches) and were checked carefully for reproducibility. Background spectra were obtained on clean solvents at the same measurement conditions. 
Spectral simulation was performed using the program QCMP 136 by Prof. Dr. Frank Neese from the Quantum Chemistry Program Exchange as used by Neese et al. ${ }^{1}$ The fittings were performed by the "chi by eye" approach and always taking all available spectra at different temperatures into account. Collinear $g$ and $A$ tensors were used. Spectral simulations were performed using the simple $1 \mathrm{Cu}, 2 \mathrm{~N}$ model (nuclear spin for ${ }^{63 / 65} \mathrm{Cu}: I=3 / 2$ and for $\left.{ }^{14} \mathrm{~N}: I=1\right)$. The $A(\mathrm{~N})$ values used are only estimates and contribute to the line broadening since resolved hyperfine to ${ }^{14} \mathrm{~N}$ atoms are not explicitly observed in the EPR spectra of $\mathbf{2}$ and $\mathbf{3}$.

\section{Materials.}

All chemicals were purchased from common vendors (e.g. Sigma-Aldrich, Acros Organics, Strem Chemicals, TCI) and used without further purification unless otherwise mentioned. TMS-acetylene was purchased from combi-blocks chemicals. Molecular sieves (4A, 4-8 mesh beads) were obtained from Fisher Scientific and activated in vacuo at $200{ }^{\circ} \mathrm{C}$ for $24 \mathrm{~h}$. Extra dry solvents ( $\geq 99.5 \%$ ) with Acroseal ${ }^{\circledR}$ and deuterated solvents were purchased from Acros Organics and Cambridge Isotope Laboratories, respectively. Both anhydrous and deuterated solvents were sparged with nitrogen and stored over activated $4 \AA$ molecular sieves under a nitrogen atmosphere. 4-Ethynyltoluene, 4-tertbutylphenylacetylene, 1-ethynyl-4-fluorobenzene, 2-ethynylnaphthalene, 2ethynylthiophene, and 1-ethynyl-4-trifluoromethylbenzene were purchased from Sigma-Aldrich and purified prior to use. Terminal alkynes that were not commercially available were synthesized according to literature. ${ }^{2}$

$\left.\mathrm{CuO}^{t} \mathrm{Bu}^{3}{ }^{3} \quad \mathrm{H}^{i} \mathrm{Pr}_{2} \mathrm{NN}\right]^{4}, \quad\left[{ }^{i} \mathrm{Pr}_{2} \mathrm{NN}\right] \mathrm{Cu}(\mathrm{NCMe})^{5} \quad(\mathbf{1 - M e C N}), \quad\left[\mathrm{Me}_{3} \mathrm{NN}\right] \mathrm{Cu}^{6}$, $\left[\mathrm{Cl}_{2} \mathrm{NN}_{\mathrm{F} 6}\right] \mathrm{Cu}^{7}$, and $\left[\mathrm{IPr}_{2}{ }_{2} \mathrm{NN}\right] \mathrm{Cu}^{8}$ were synthesized and characterized according to literature procedures. 


\section{Synthesis and Characterization of $\left[{ }^{i} \mathrm{Pr}_{2} \mathrm{NN}\right] \mathrm{Cu}-\mathrm{O}^{t} \mathrm{Bu}(2)$.}
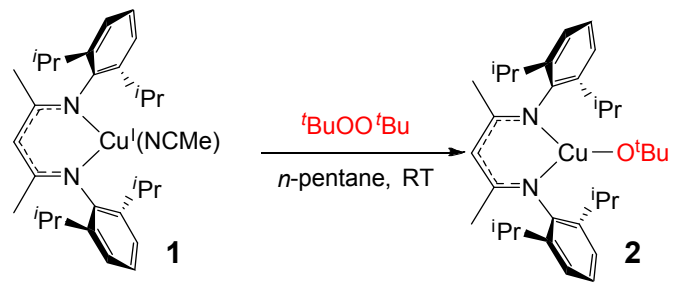

$73 \%$ as brown crystals

Scheme S1. Synthesis of $\left[{ }^{i} \mathrm{Pr}_{2} \mathrm{NN}\right] \mathrm{Cu}-\mathrm{O}^{t} \mathrm{Bu}(2)$.

$\left[{ }^{i} \mathrm{Pr}_{2} \mathrm{NN}\right] \mathrm{Cu}(\mathrm{NCMe})(\mathbf{1 - M e C N})$ was synthesized by a modification of a literature procedure. ${ }^{4}$ To a chilled solution of $\mathrm{H}\left[{ }^{i} \mathrm{Pr}_{2} \mathrm{NN}\right](1.00 \mathrm{~g}, 2.39 \mathrm{mmol})$ in anhydrous THF (ca. $10 \mathrm{~mL}$ ) was added a solution of $n-\operatorname{BuLi}(2.5 \mathrm{M}$ in hexanes, $1.00 \mathrm{~mL}, 2.51 \mathrm{mmol})$ dropwise and the reaction mixture was stirred at RT for $3 \mathrm{~h}$. Then, $\left[\mathrm{Cu}\left(\mathrm{NCCH}_{3}\right)_{4}\right] \mathrm{CF}_{3} \mathrm{SO}_{3}$ $(0.901 \mathrm{~g}, 2.39 \mathrm{mmol})$ was added to the resultant yellow solution and the reaction mixture was stirred at RT for another $10 \mathrm{~min}$. All volatiles were then removed under vacuum and the resultant greenish yellow residue was then extracted twice with $10 \mathrm{~mL}$ anhydrous $n$-pentane. After filtration through a plug of Celite, all volatiles were removed under dynamic vacuum to obtain a bright yellow solid. This solid was recrystallized from $n$-pentane at $-35{ }^{\circ} \mathrm{C}$ to afford colorless crystals of $\left[{ }^{i} \operatorname{Pr}_{2} \mathrm{NN}\right] \mathrm{Cu}(\mathrm{NCMe})(\mathbf{1}-\mathrm{MeCN})$ in $82 \%$ yield $(1.02 \mathrm{~g}, 1.96 \mathrm{mmol})$. Spectroscopic data matches those reported previously. ${ }^{5}$

$\left[{ }^{i} \mathrm{Pr}_{2} \mathrm{NN}\right] \mathrm{Cu}-\mathrm{O}^{t} \mathrm{Bu}$ (2) was synthesized from the corresponding copper(I) $\beta$-diketiminate 1-MeCN in a related manner to other $\beta$-diketiminato copper(II) tert-butoxide complexes. ${ }^{6,-10} \mathrm{Di}$-tert-butyl peroxide $(1.31 \mathrm{~mL}, 7.13 \mathrm{mmol})$ was added dropwise to a chilled suspension of $\left[{ }^{i} \operatorname{Pr}_{2} \mathrm{NN}\right] \mathrm{Cu}(\mathrm{NCMe})(0.750 \mathrm{~g}, 1.43 \mathrm{mmol})$ in $15 \mathrm{~mL}$ anhydrous $n$-pentane. The reaction mixture was stirred at RT for $3 \mathrm{~h}$. The resulting brown solution was filtered through a pad of Celite and the solvent was evaporated under reduced pressure to obtain 2 as a dark brown solid $(0.569 \mathrm{~g}, 1.03 \mathrm{mmol})$ in $73 \%$ yield. $\mathrm{X}$-ray quality crystals were grown from a concentrated $n$-pentane solution of 2 at $-35^{\circ} \mathrm{C}$. $\mathrm{UV}-\mathrm{V}$ is (toluene, $25{ }^{\circ} \mathrm{C}$ ): $\lambda_{\max } / \mathrm{nm}\left(\varepsilon / \mathrm{M}^{-1} \mathrm{~cm}^{-1}\right)=470$ (1450) (Figure S1). EPR (toluene, $293 \mathrm{~K}): g_{\text {iso }}=2.11$ with $A_{\text {iso }}(\mathrm{Cu})=115.0 \mathrm{MHz}$ and $A_{\text {iso }}(\mathrm{N})=30.0 \mathrm{MHz}$ (Figure S2). EPR (toluene, $80 \mathrm{~K}$ ): $g_{1}=2.21, g_{2}=2.07, g_{3}=2.04$ with $A_{1}(\mathrm{Cu})=330.0, A_{2}(\mathrm{Cu})=5.0, A_{3}(\mathrm{Cu})$ $=0 \mathrm{MHz}$ and $A_{1}(\mathrm{~N})=0.0, A_{2}(\mathrm{~N})=75.0, A_{3}(\mathrm{~N})=33.0 \mathrm{MHz}$ (Figure $\mathrm{S} 3$ ). Anal. Calcd for $\mathrm{C}_{33} \mathrm{H}_{50} \mathrm{~N}_{2} \mathrm{OCu}(1):$ C, 71.50; H, 9.09; N, 5.05. Found: C, 71.76; H, 9.16; N, 5.09. 

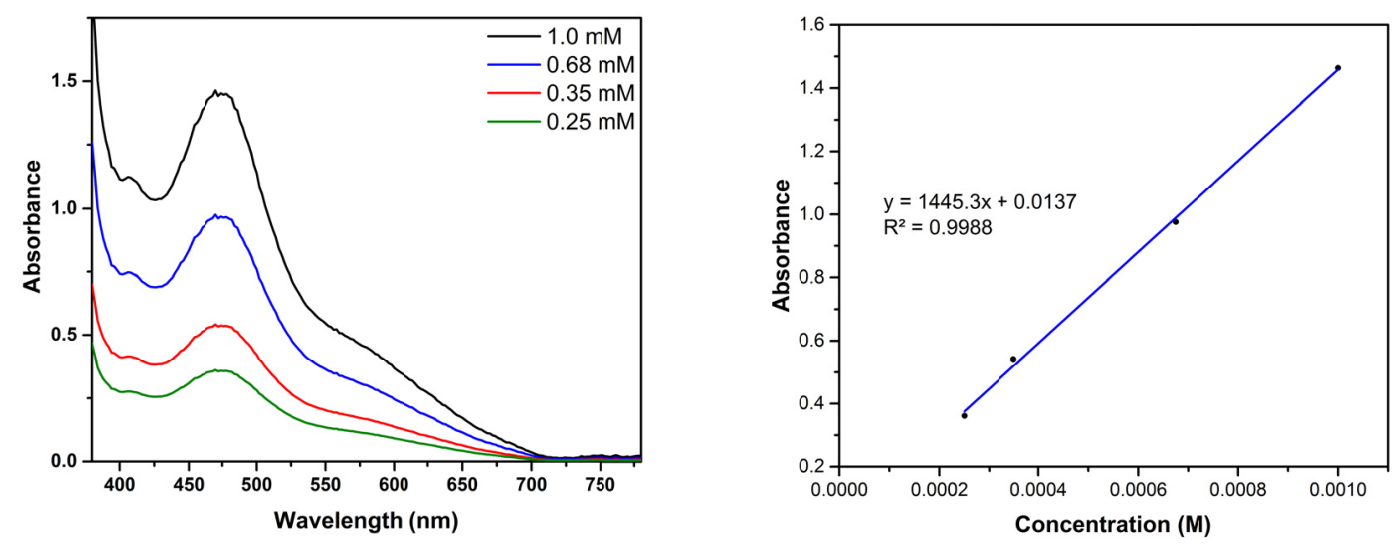

Figure S1. Left: UV-Vis spectra of 2 in toluene at $25{ }^{\circ} \mathrm{C}$ at different concentrations. Right: Beer's law plot for 2 depicts $\lambda_{\max } / \mathrm{nm}\left(\varepsilon / \mathrm{M}^{-1} \mathrm{~cm}^{-1}\right)=470(1450)$.

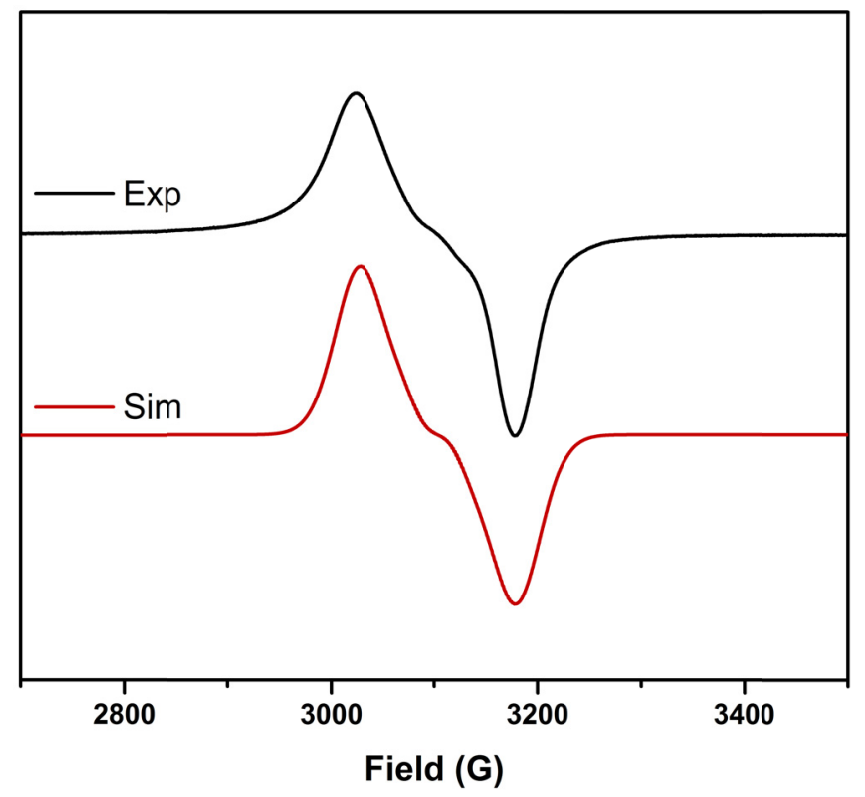

Figure S2. $\mathrm{X}$-band EPR spectrum and simulation for $\left[{ }^{i} \mathrm{Pr}_{2} \mathrm{NN}\right] \mathrm{Cu}-\mathrm{O}^{t} \mathrm{Bu}$ (2) (toluene, $293 \mathrm{~K}$, Frequency $=9.162439 \mathrm{GHz}$, power $=1.0 \mathrm{~mW}$, ModWidth $=1.6 \mathrm{mT}$, time - constant $=0.001 \mathrm{~s}$ ). Simulation was performed using a $1 \mathrm{Cu}, 2 \mathrm{~N}$ model: $g_{\text {iso }}=2.11$, with $A_{\text {iso }}(\mathrm{Cu})=112.0 \mathrm{MHz}$ and $A_{\text {iso }}(\mathrm{N})=5.0 \mathrm{MHz}$, Gaussian line shape with line broadening of $W_{\text {iso }}=28 \mathrm{mT}$. 


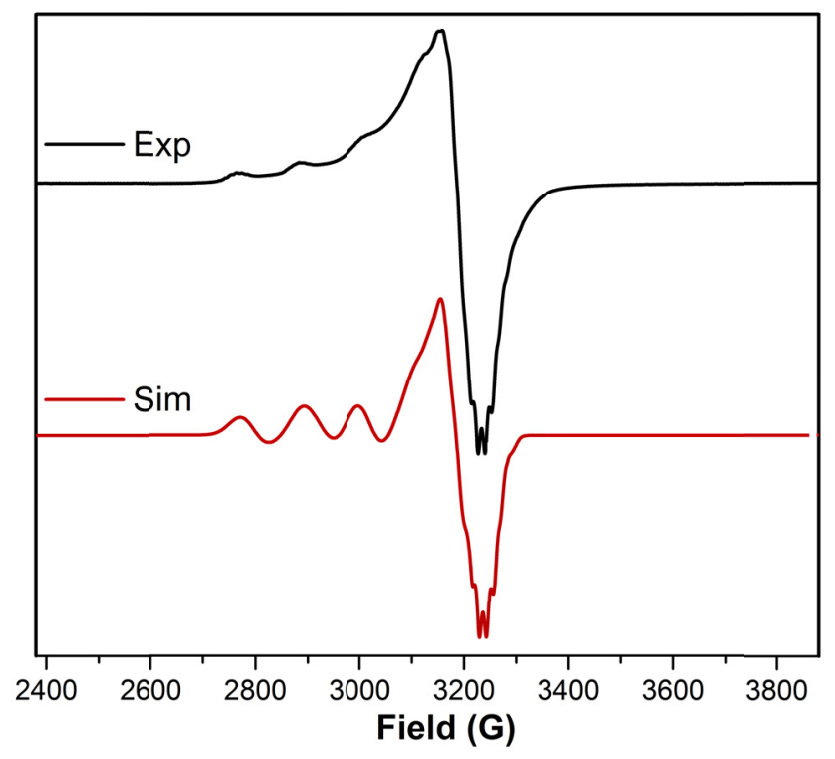

Figure S3. $\mathrm{X}$-band EPR spectrum and simulation for $\left[{ }^{i} \mathrm{Pr}_{2} \mathrm{NN}\right] \mathrm{Cu}-\mathrm{O}{ }^{t} \mathrm{Bu}$ (2) (toluene, 80 $\mathrm{K}$, Frequency $=9.181759 \mathrm{GHz}$, power $=0.998 \mathrm{~mW}$, ModWidth $=1.0 \mathrm{mT}$, time - constant $=0.01 \mathrm{~s}$ ). Simulation was performed using a $1 \mathrm{Cu}, 2 \mathrm{~N}$ model: $g_{1}=2.225, g_{2}=2.070, g_{3}=$ 2.035 with $A_{1}(\mathrm{Cu})=330.00, A_{2}(\mathrm{Cu})=0.00, A_{3}(\mathrm{Cu})=6.00 \mathrm{MHz}$ and $A_{1}(\mathrm{~N})=0.00, A_{2}(\mathrm{~N})=$ $70.00, A_{3}(\mathrm{~N})=70.00 \mathrm{MHz}$, Gaussian line shape with line broadening of $W_{1}=30, W_{2}=16$, $W_{3}=13 \mathrm{mT}$. 


\section{Synthesis and Characterization of $\left[{ }^{i} \operatorname{Pr}_{2} \mathrm{NN}\right] \mathrm{Cu}-\mathrm{C} \equiv \mathrm{CAr}{ }^{\mathrm{Cl} 2}(3)$.}

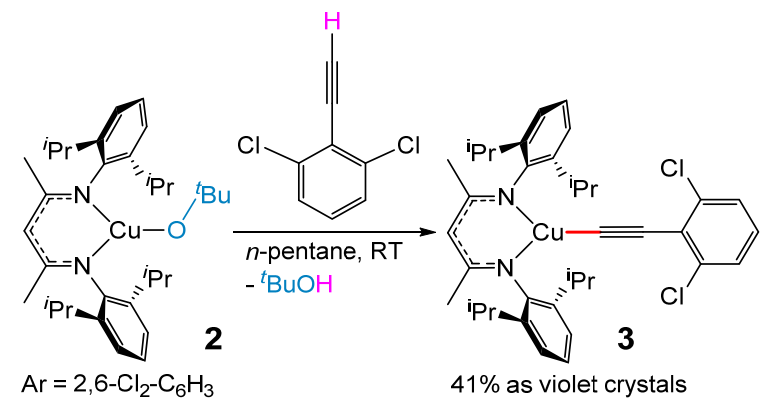

Scheme S2. Synthesis of $\left[{ }^{i} \operatorname{Pr}_{2} \mathrm{NN}\right] \mathrm{Cu}-\mathrm{C} \equiv \mathrm{CAr}^{\mathrm{Cl} 2}$ (3).

A solution of ${ }^{\mathrm{Cl} 2} \mathrm{ArC} \equiv \mathrm{CH}(0.233 \mathrm{~g}, 1.36 \mathrm{mmol})$ in $n$-pentane $(10 \mathrm{~mL})$ was added to a solution of $2(0.755 \mathrm{~g}, 1.36 \mathrm{mmol})$ in $n$-pentane $(5 \mathrm{~mL})$. The color of the solution slowly changed from dark brown to dark violet. After stirring for $45 \mathrm{~min}$, the resultant mixture was filtered through a plug of Celite and concentrated under reduced pressure. Allowing the resulting solution to stand at $-35^{\circ} \mathrm{C}$ for a week afforded dark violet crystals of the $\mathrm{Cu}$ (II) alkynyl complex 3 in $41 \%$ yield $(0.364 \mathrm{~g}, 0.558 \mathrm{mmol})$. Satisfactory elemental analysis data could not be obtained due to the thermal sensitivity of compound 3. UV-Vis (heptane, $\left.25^{\circ} \mathrm{C}\right): \lambda_{\max } / \mathrm{nm}\left(\varepsilon / \mathrm{M}^{-1} \mathrm{~cm}^{-1}\right)=500$ (4150), 915 (405) (Figure $\left.\mathrm{S} 4\right)$. EPR (toluene, $200 \mathrm{~K}$ ): $g_{\text {iso }}=2.1$ with $A_{\text {iso }}(\mathrm{Cu})=210.0 \mathrm{MHz}$ and $A_{\text {iso }}(\mathrm{N})=40.0 \mathrm{MHz}$ (Figure S5). EPR (toluene, $80 \mathrm{~K}$ ): $g_{1}=2.18, g_{2}=2.04, g_{3}=2.05$ with $A_{1}(\mathrm{Cu})=130.0$, $A_{2}(\mathrm{Cu})=290.0, A_{3}(\mathrm{Cu})=130.0 \mathrm{MHz}$ and $A_{1}(\mathrm{~N})=45.0 \mathrm{MHz}, A_{2}(\mathrm{~N})=A_{3}(\mathrm{~N})=35.0 \mathrm{MHz}$ (Figure S6). FT-IR (KBr pellet, $\mathrm{cm}^{-1}$ ): 2187 [ $\left.v_{\mathrm{C} \equiv \mathrm{C}}\right]$ (Figure S7). 

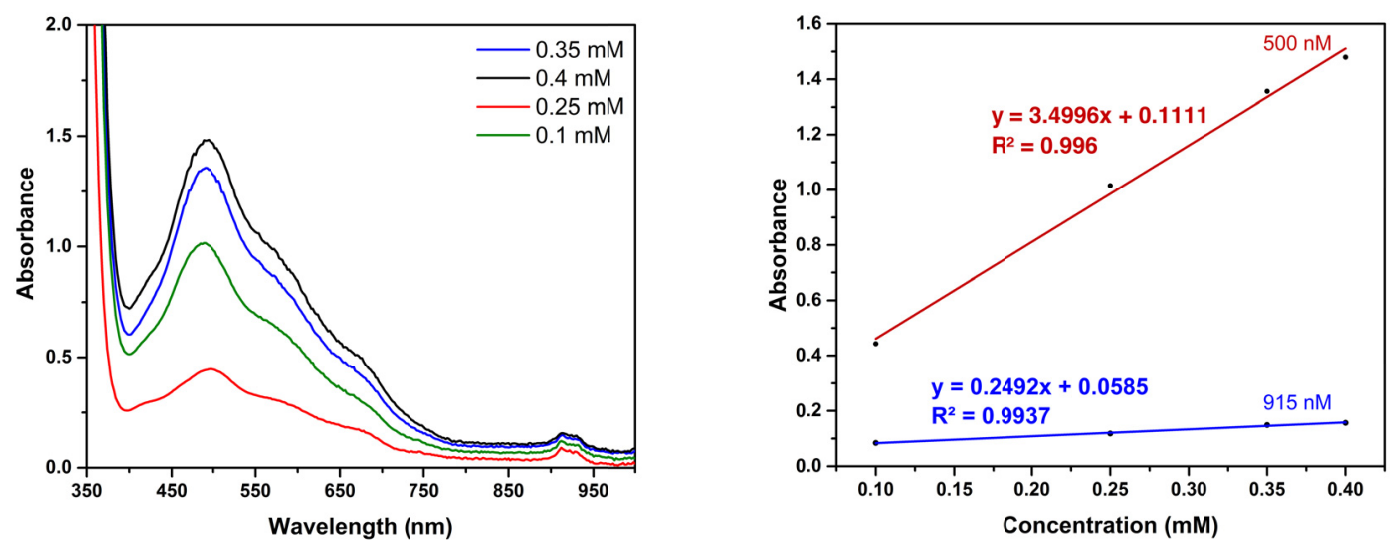

Figure S4. Left: UV-Vis spectra of 3 in toluene at $25{ }^{\circ} \mathrm{C}$ at different concentrations. Right: Beer's law plot for $2^{\mathrm{Me}}$ depicts $\lambda_{\max } / \mathrm{nm}\left(\varepsilon / \mathrm{M}^{-1} \mathrm{~cm}^{-1}\right)=500$ (3500), 915 (250).

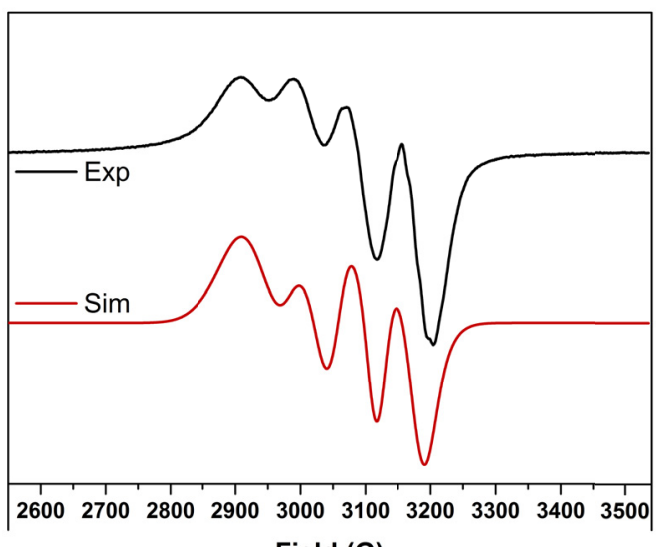

Field (G)

Figure S5. $\mathrm{X}$-band EPR spectrum and simulation for $\left[{ }^{i} \operatorname{Pr}_{2} \mathrm{NN}\right] \mathrm{Cu}-\mathrm{C} \equiv \mathrm{CAr}{ }^{\mathrm{Cl} 2}$ (3) (toluene, $200 \mathrm{~K}$, Frequency $=8.968117 \mathrm{GHz}$, power $=0.455 \mathrm{~mW}$, ModWidth $=2 \mathrm{mT}$, time - constant $=0.001 \mathrm{~s}$ ). Simulation was performed using a $1 \mathrm{Cu}, 2 \mathrm{~N}$ model: $g_{\text {iso }}=2.1$, with $A_{\text {iso }}(\mathrm{Cu})=210.0 \mathrm{MHz}$ and $A_{\text {iso }}(\mathrm{N})=40.0 \mathrm{MHz}$, Gaussian line shape with line broadening of $W_{\text {iso }}=31 \mathrm{mT}$. 


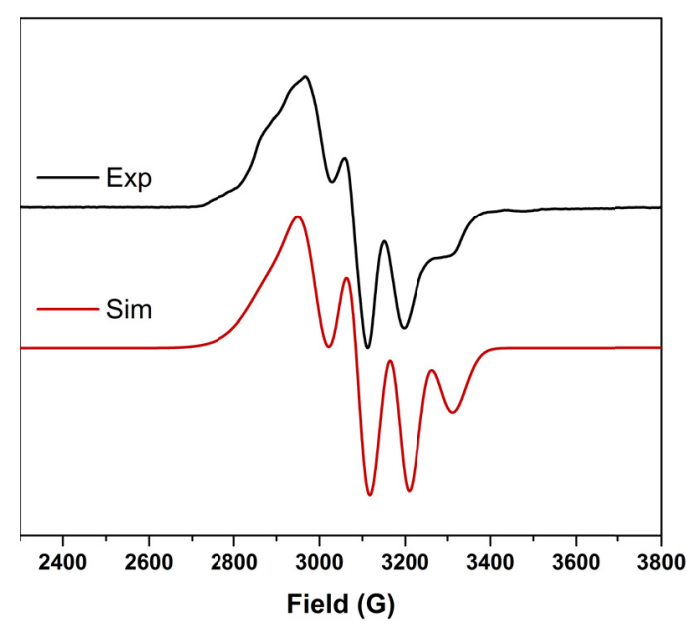

Figure S6. $\mathrm{X}$-band EPR spectrum and simulation for $\left[{ }^{i} \mathrm{Pr}_{2} \mathrm{NN}\right] \mathrm{Cu}-\mathrm{C} \equiv \mathrm{CAr}^{\mathrm{Cl} 2}$ (3) (toluene, $80 \mathrm{~K}$, Frequency $=8.968114 \mathrm{GHz}$, power $=1 \mathrm{~mW}$, ModWidth $=1.0 \mathrm{mT}$, time - constant $=0.01 \mathrm{~s}$ ). Simulation was performed using a $1 \mathrm{Cu}, 2 \mathrm{~N}$ model: $g_{1}=2.18, g_{2}=2.04, g_{3}=$ 2.05 with $A_{1}(\mathrm{Cu})=130, A_{2}(\mathrm{Cu})=290, A_{3}(\mathrm{Cu})=130 \mathrm{MHz}$ and $A_{1}(\mathrm{~N})=45 \mathrm{MHz}, A_{2}(\mathrm{~N})=$ $A_{3}(\mathrm{~N})=35.0 \mathrm{MHz}$, Gaussian line shape with line broadening of $W_{1}=60, W_{2}=20, W_{3}=$ $50 \mathrm{mT}$.

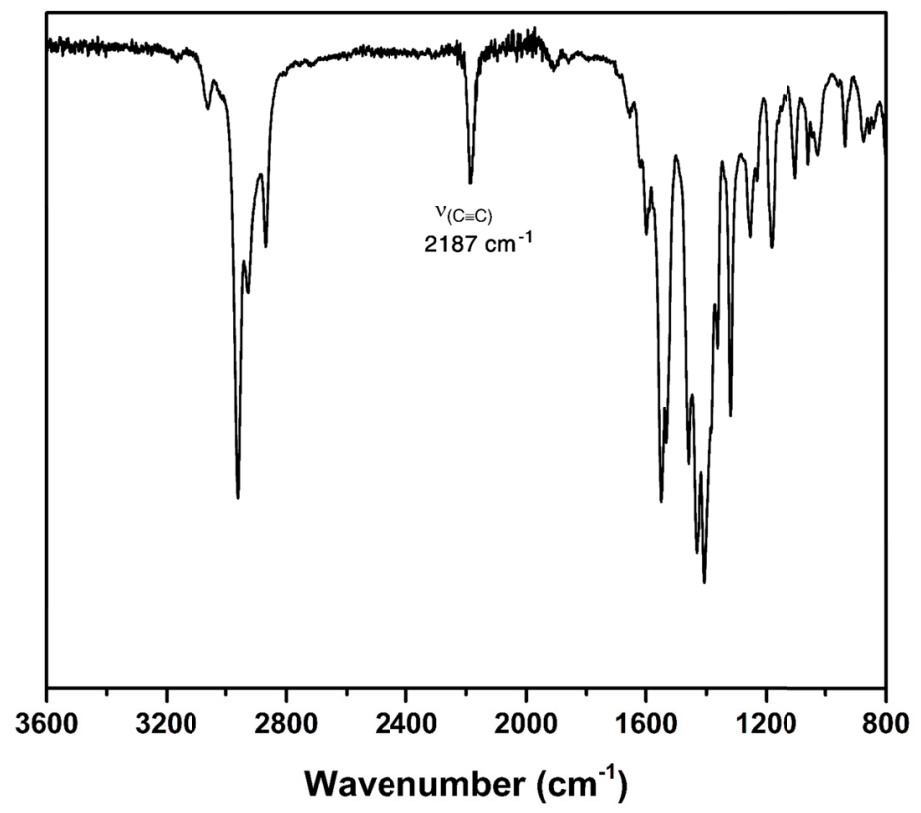

Figure S7. FT-IR (KBr pellet) spectrum of $\left[{ }^{i} \operatorname{Pr}_{2} \mathrm{NN}\right] \mathrm{Cu}-\mathrm{C} \equiv \mathrm{CAr}^{\mathrm{Cl} 2} \cdot v_{(\mathrm{C} \equiv \mathrm{C})}=2187 \mathrm{~cm}^{-1}$. The free alkyne $\mathrm{H}-\mathrm{C} \equiv \mathrm{CAr}{ }^{\mathrm{Cl} 2}$ shows $v_{(\mathrm{C} \equiv \mathrm{C})}$ at $2099 \mathrm{~cm}^{-1}$ (previously reported $v_{(\mathrm{C} \equiv \mathrm{C})}=$ $\left.2101 \mathrm{~cm}^{-1}\right)^{2}$. 


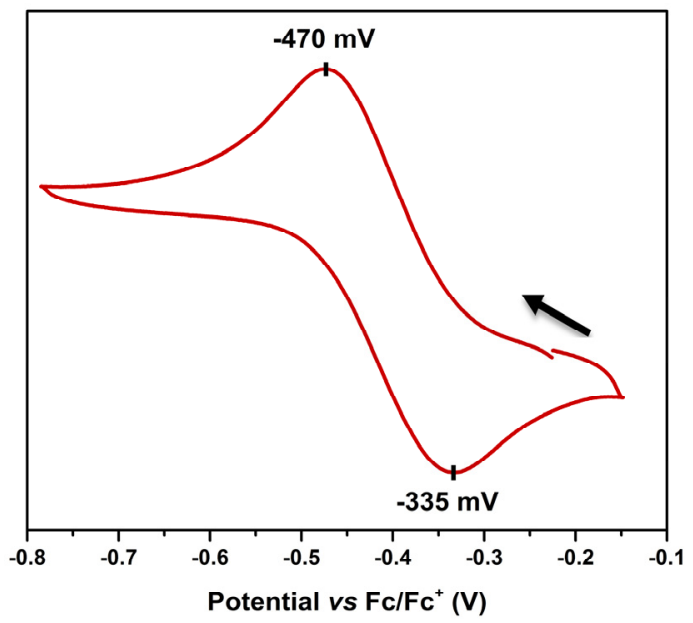

Figure S8. Cyclic voltammogram of $\left[{ }^{i} \mathrm{Pr}_{2} \mathrm{NN}\right] \mathrm{Cu}-\mathrm{C} \equiv \mathrm{CAr}^{\mathrm{Cl} 2} \quad$ (3) $\quad(2.5 \mathrm{mM}$ in fluorobenzene at $\left.25{ }^{\circ} \mathrm{C}\right)$ in the presence of sodium tetrakis[(3,5-trifluoromethyl)phenyl]borate $(0.1 \mathrm{M})$. Scan proceeds in the indicated direction with scan rate of $20 \mathrm{mV} / \mathrm{s}$. 


\section{Observation of $\left.\left[\mathrm{CoCp}_{2}\right]^{+}\left\{{ }^{i} \mathrm{Pr}_{2} \mathrm{NN}\right] \mathrm{Cu}-\mathrm{C} \equiv \mathrm{CAr}^{\mathrm{Cl} 2}\right\}^{-}\left(\mathrm{Cp}_{2} \mathrm{Co}^{+}-4\right)$.}

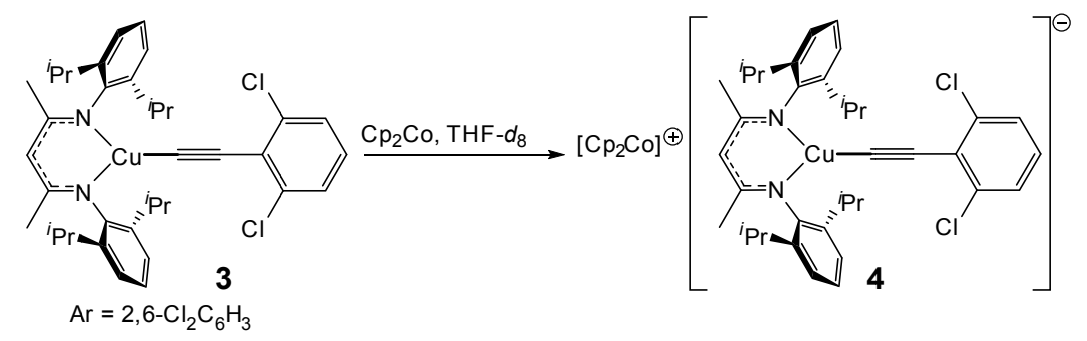

Scheme S3. Observation of $\left.\left[\mathrm{CoCp}_{2}\right]^{+}\left\{{ }^{i} \mathrm{Pr}_{2} \mathrm{NN}\right] \mathrm{Cu}-\mathrm{C} \equiv \mathrm{CAr}^{\mathrm{Cl} 2}\right\}^{-}\left(\mathbf{C p}_{2} \mathbf{C o}^{+}-4\right)$ in $\mathrm{THF}-d_{8}$.

A solution of $\left[{ }^{i} \mathrm{Pr}_{2} \mathrm{NN}\right] \mathrm{Cu}-\mathrm{C} \equiv \mathrm{CAr}^{\mathrm{Cl} 2}(\mathbf{3})(0.1 \mathrm{~g}, 0.154 \mathrm{mmol})$ in $\mathrm{THF}-d_{8}(1.0 \mathrm{~mL})$ was added to a solution of cobaltocene $\left(\mathrm{Cp}_{2} \mathrm{Co}\right)(0.029 \mathrm{~g}, 0.154 \mathrm{mmol})$ in $\mathrm{THF}-d_{8}(1.0$ $\mathrm{mL}$ ) and stirred for $5 \mathrm{~min}$. The color of the reaction mixture immediately changed from violet to orange with concomitant precipitation of some insoluble yellow solid. While anionic complex $\mathbf{C p}_{2} \mathbf{C o}^{+}-4$ maybe observed by ${ }^{1} \mathrm{H}$ NMR spectroscopy in THF- $d_{8}$, a yellow solid forms concurrently that is not soluble in THF- $d_{8}$ or other common deutrated NMR solvents. ${ }^{1} \mathrm{H}$ NMR (400 MHz, $\left.298 \mathrm{~K}, \mathrm{THF}-d_{8}\right): \delta 6.60$ (d, $\left.J=8.2 \mathrm{~Hz}, 2 \mathrm{H}\right), 6.17$ (t, $J$ $=8.1 \mathrm{~Hz}, 1 \mathrm{H}), 4.75(\mathrm{~s}, 1 \mathrm{H}), 3.95(\mathrm{~d}, J=1.1 \mathrm{~Hz}, 10 \mathrm{H}), 3.26($ hept, $J=7.1 \mathrm{~Hz}, 4 \mathrm{H}), 1.61(\mathrm{~s}$, $6 \mathrm{H}), 1.16(\mathrm{~d}, J=6.9 \mathrm{~Hz}, 12 \mathrm{H}), 1.11$ (d, $J=6.9 \mathrm{~Hz}, 12 \mathrm{H})$.
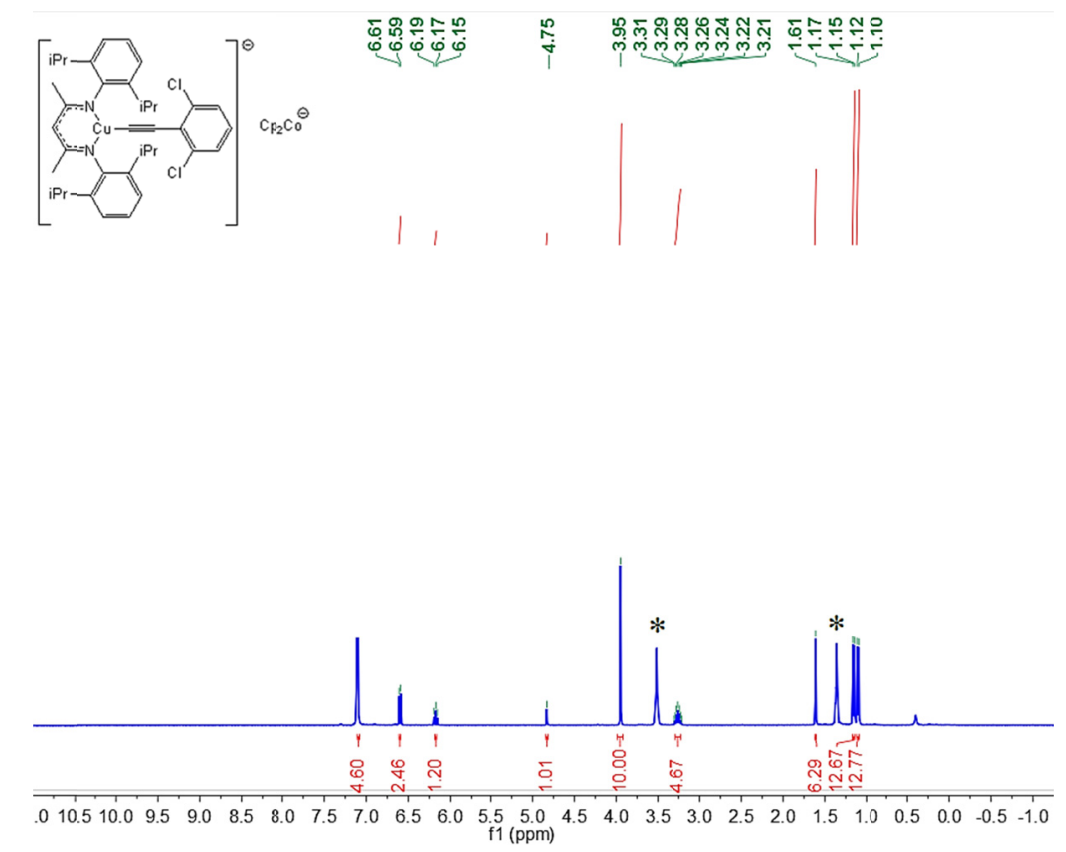

Figure S9. ${ }^{1} \mathrm{H}$ NMR spectrum $\left(400 \mathrm{MHz}, 298 \mathrm{~K}, \mathrm{THF}-d_{8}\right)$ of $\left(\mathbf{C p}_{2} \mathbf{C o}{ }^{+}-4\right)$. The resonances marked with $*$ are proteo impurities of $\mathrm{THF}-d_{8}$. 


\section{Thermal Decay of $\left[{ }^{i} \mathrm{Pr}_{2} \mathrm{NN}\right] \mathrm{Cu}-\mathrm{C} \equiv \mathrm{CAr}^{\mathrm{Cl} 2}$ (3).}

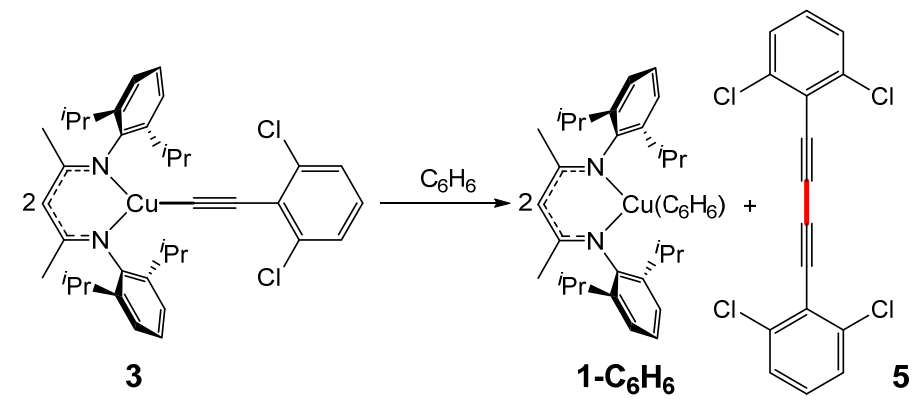

Scheme S4. Thermal decay of $\left[{ }^{i} \mathrm{Pr}_{2} \mathrm{NN}\right] \mathrm{Cu}-\mathrm{C} \equiv \mathrm{CAr}^{\mathrm{Cl} 2}(\mathbf{3})$ in $\mathrm{C}_{6} \mathrm{H}_{6}$ at $\mathrm{RT}$ and $90{ }^{\circ} \mathrm{C}$.

Solutions of $\left[{ }^{i} \operatorname{Pr}_{2} \mathrm{NN}\right] \mathrm{Cu}-\mathrm{C} \equiv \mathrm{CAr}^{\mathrm{Cl} 2}$ (3) in benzene $(1.0 \mathrm{~mL}, 10.0 \mathrm{mM}, 0.01$ mmol) and 1,4-dibromo-2,5-difluorobenzene in benzene (1.0 mL, $10.0 \mathrm{mM}, 0.01$ mmol) were taken in a scintillation vial inside a glovebox. The vial was then sealed and stirred at RT until the violet color bleached (ca. $180 \mathrm{~min}$ ). The resultant reaction mixture was analyzed without any further workup via GC-MS analysis on a dilute solution in dichloromethane that confirms the formation of ${ }^{\mathrm{Cl} 2} \mathrm{ArC} \equiv \mathrm{C}-\mathrm{C} \equiv \mathrm{CAr}{ }^{\mathrm{Cl} 2}$ in $68 \%$ yield.

In another experiment, solutions of $\left[{ }^{i} \mathrm{Pr}_{2} \mathrm{NN}\right] \mathrm{Cu}-\mathrm{C} \equiv \mathrm{CAr}{ }^{\mathrm{Cl} 2}(\mathbf{3})$ in benzene $(1.0 \mathrm{~mL}$, $10.0 \mathrm{mM}, 0.01 \mathrm{mmol})$ and 1,4-dibromo-2,5-difluorobenzene in benzene $(1.0 \mathrm{~mL}, 10.0$ $\mathrm{mM}, 0.01 \mathrm{mmol}$ ) were taken in a pressure vessel. The pressure vessel was then sealed and heated to $90{ }^{\circ} \mathrm{C}$ for $1 \mathrm{~h}$. The color of the reaction mixture changed from violet to bright yellow with formation of an insoluble yellow solid. The reaction mixture was then filtered through a plug of Celite and was analyzed without any further workup via GC-MS. GC-MS analysis on a dilute solution in dichloromethane confirms the formation of ${ }^{\mathrm{Cl} 2} \mathrm{ArC} \equiv \mathrm{C}-\mathrm{C} \equiv \mathrm{CAr}^{\mathrm{Cl} 2}$ in $89 \%$ yield. 

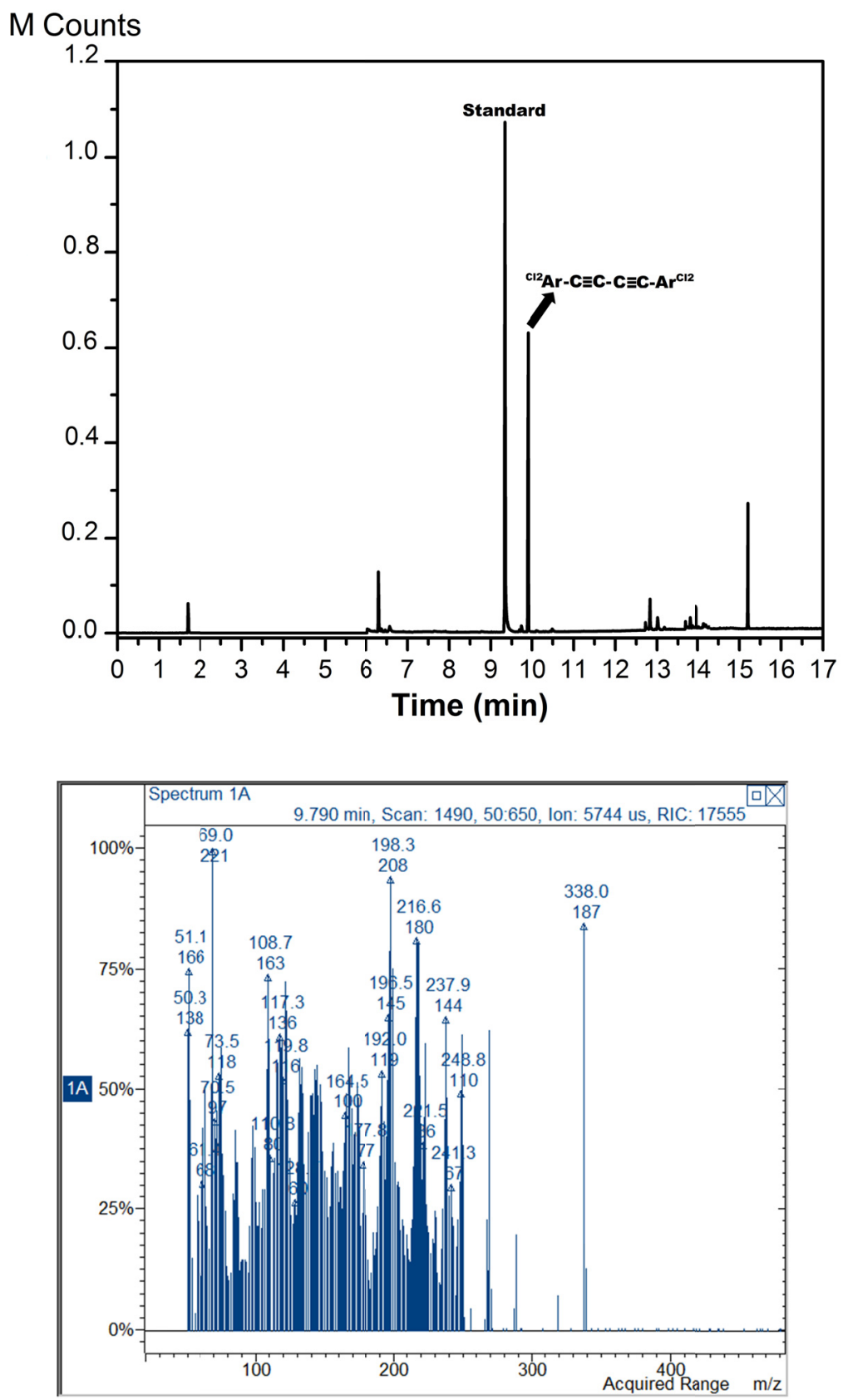

Figure S10. GC-MS (EI mode) profile of the crude reaction mixture obtained upon decay of $\left[{ }^{i} \mathrm{Pr}_{2} \mathrm{NN}\right] \mathrm{Cu}-\mathrm{C} \equiv \mathrm{CAr}^{\mathrm{Cl} 2} \quad$ (3) $\quad\left(\begin{array}{llllll}1.0 & \mathrm{~mL}, & 10.0 & \mathrm{mM}, & 0.01 & \mathrm{mmol}\end{array}\right)$ and 1,4-dibromo-2,5-difluorobenzene (as internal standard) in $\mathrm{C}_{6} \mathrm{H}_{6}$. Top: GC-FID plot showing 1,3-diyne 5 (retention time: $9.790 \mathrm{~min}$ ) and 1,4-dibromo-2,5-difluorobenzene (retention time: $9.202 \mathrm{~min}$ ). Bottom: MS-EI mode spectra of 1,4-bis(2,6-dichlorophenyl)buta-1,3-diyne (5) (calc. $m / z$ for $\mathrm{C}_{16} \mathrm{H}_{6} \mathrm{Cl}_{4}$ : 337.92). 


\section{Decay of $\left[{ }^{i} \mathrm{Pr}_{2} \mathrm{NN}\right] \mathrm{Cu}-\mathrm{C} \equiv \mathrm{CAr}^{\mathrm{Cl2}}$ (3) Promoted by Acetonitrile and 2,4-Lutidine.}

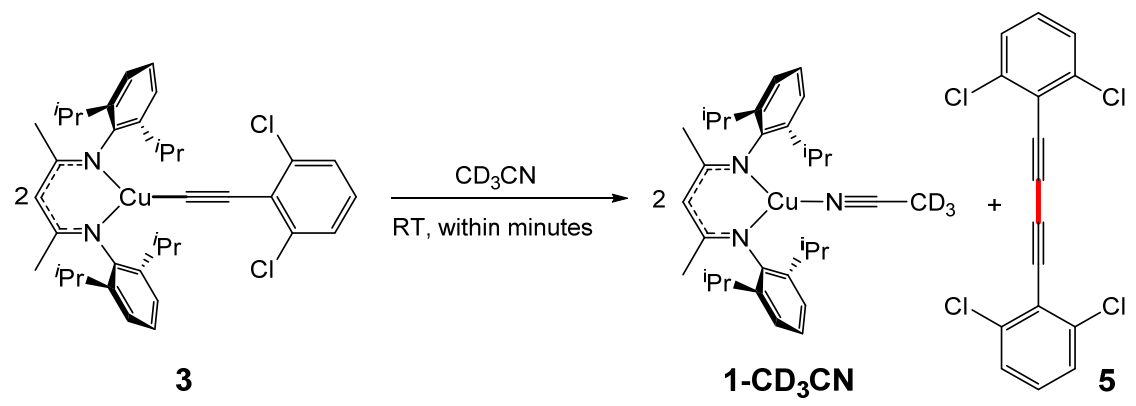

Scheme S5. Reaction of 3 with $\mathrm{CD}_{3} \mathrm{CN}$ at $25^{\circ} \mathrm{C}$.

Dark violet crystals of $3(0.017 \mathrm{~g}, 0.026 \mathrm{mmol})$ and freshly sublimed, anhydrous ferrocene as internal standard $(4.84 \mathrm{mg}, 0.026 \mathrm{mmol})$ were dissolved in rigorously anhydrous $\mathrm{CD}_{3} \mathrm{CN}(0.5 \mathrm{~mL})$. The color of the solution immediately turned orange with concomitant formation of a dark orange solid. The solution became bright yellow within $40 \mathrm{~min}$, at which point the resultant mixture was filtered through a plug of Celite to give a bright yellow solution. This solution was analyzed by ${ }^{1} \mathrm{H}$ NMR spectroscopy (Figure S11) as well as GC-MS. ${ }^{1} \mathrm{H}$ NMR analysis in presence of ferrocene as internal standard indicates formation of copper(I) complex 1-MeCN (98\% yield) and Glaser homocoupling product ${ }^{\mathrm{C} 12} \mathrm{Ar} \equiv \mathrm{C}-\mathrm{C} \equiv \mathrm{CAr}{ }^{\mathrm{Cl} 2}$ in $88 \%$. GC-MS analysis on a dilute solution confirms the formation of ${ }^{\mathrm{C} 12} \mathrm{Ar} \equiv \mathrm{C}-\mathrm{C} \equiv \mathrm{CAr}{ }^{\mathrm{C} 12}$ product.

On a similar scale, excess 2,4-lutidine (ca. 5 equiv.) was added to a dark violet solution of 3 in $n$-pentane $(1 \mathrm{~mL})$ containing ferrocene as an internal standard. The solution immediately turned orange with formation of a yellow solid $\left({ }^{\mathrm{Cl} 2} \mathrm{Ar} \equiv \mathrm{C}-\mathrm{C} \equiv \mathrm{CAr}{ }^{\mathrm{Cl} 2}\right)$, changing to bright yellow within $5 \mathrm{~min}$. After removal of volatiles, analysis by ${ }^{1} \mathrm{H}$ NMR in benzene- $d_{6}$ revealed near quantitative formation of the Glaser homocoupling product ${ }^{\mathrm{Cl} 2} \mathrm{Ar}=\mathrm{C}-\mathrm{C} \equiv \mathrm{CAr}{ }^{\mathrm{Cl} 2}$, also confirmed by GC-MS analysis. 


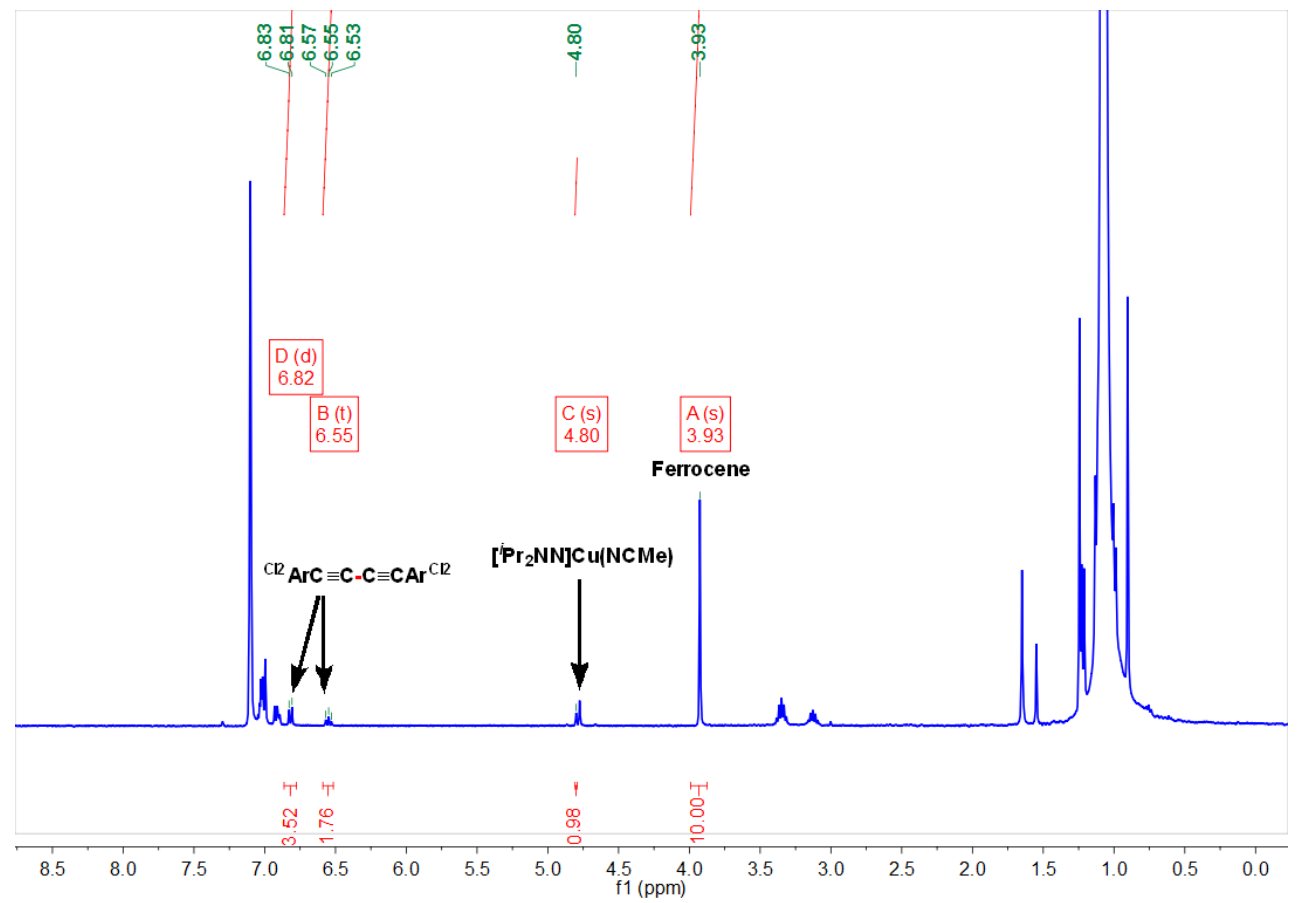

Figure S11. ${ }^{1} \mathrm{H}$ NMR spectrum $\left(400 \mathrm{MHz}, 298 \mathrm{~K}, \mathrm{CD}_{3} \mathrm{CN}\right)$ of 3 that has undergone immediate conversion to $\left[{ }^{\mathrm{i}} \mathrm{Pr}_{2} \mathrm{NN}\right] \mathrm{Cu}(\mathrm{NCMe})$ and ${ }^{\mathrm{Cl} 2} \mathrm{Ar} \equiv \mathrm{C}-\mathrm{C} \equiv \mathrm{CAr}{ }^{\mathrm{Cl} 2}$. The resonance at $\delta$ $1.94 \mathrm{ppm}$ is the solvent residual peak of acetonitrile. Internal standard (ferrocene) shows a singlet at $3.93 \mathrm{ppm}$. The $\beta$-diketiminate backbone $\mathrm{C}-\mathrm{H}$ signal was used to determine the yield of 1-MeCN (signal at $\delta 4.80 \mathrm{ppm} ; 98 \%$ yield). 


\section{Reactivity of $\left[{ }^{i} \mathrm{Pr}_{2} \mathrm{NN}\right] \mathrm{Cu}-\mathrm{C} \equiv \mathrm{CAr}^{\mathrm{Cl} 2}$ (3) with Various Alkynide Anions.}

We investigated three different lithium alkynyides as nucleophiles: ${ }^{\mathrm{C} 12} \mathrm{ArC} \equiv \mathrm{CLi}$ $\left({ }^{\mathrm{Cl} 2} \mathrm{Ar}=2,6-\mathrm{Cl}_{2} \mathrm{C}_{6} \mathrm{H}_{3}\right), \mathrm{Me}_{3} \mathrm{SiC} \equiv \mathrm{CLi}$, and ${ }^{\mathrm{CF} 3} \mathrm{ArC} \equiv \mathrm{CLi}\left({ }^{\mathrm{CF} 3} \mathrm{Ar}=4-\mathrm{CF}_{3} \mathrm{C}_{6} \mathrm{H}_{4}\right)$. Addition of these lithium alkynide nucleophiles to $\left[{ }^{i} \mathrm{Pr}_{2} \mathrm{NN}\right] \mathrm{Cu}-\mathrm{C} \equiv \mathrm{CAr}^{\mathrm{Cl}}(3)$ at $-35{ }^{\circ} \mathrm{C}$ results in immediate color change from violet to dull yellow and formation of the corresponding 1,3-diynes ${ }^{\mathrm{Cl} 2} \mathrm{ArC} \equiv \mathrm{C}-\mathrm{C} \equiv \mathrm{CAr}^{\mathrm{Cl} 2}$ (5) in $71 \%, \mathrm{Me}_{3} \mathrm{SiC} \equiv \mathrm{C}-\mathrm{C} \equiv \mathrm{CAr}^{\mathrm{Cl} 2}$ (6) in $33 \%$, and ${ }^{\mathrm{CF} 3} \mathrm{ArC} \equiv \mathrm{C}-\mathrm{C} \equiv \mathrm{CAr}^{\mathrm{Cl} 2}(7)$ in $58 \%$ yields. The experimental details are provided below.

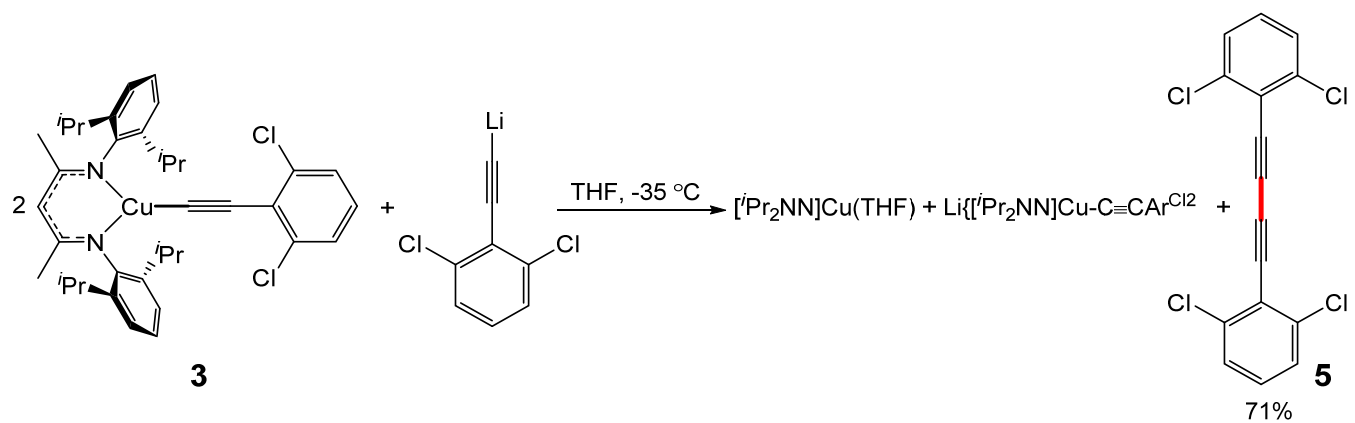

Scheme S6. Reaction of 3 with ${ }^{\mathrm{Cl} 2} \mathrm{ArC} \equiv \mathrm{C}^{-}$anion $\left({ }^{\mathrm{Cl} 2} \mathrm{Ar}=2,6-\mathrm{Cl}_{2} \mathrm{C}_{6} \mathrm{H}_{3}\right)$ in cold THF to form ${ }^{\mathrm{Cl} 2} \mathrm{ArC} \equiv \mathrm{C}-\mathrm{C} \equiv \mathrm{CAr}^{\mathrm{Cl} 2}(\mathbf{5})$.

$1000 \mu \mathrm{L} 1.5 \mathrm{mM}$ stock solution of $\mathbf{3}$ was added to a scintillation vial equipped with micro stir bar. $500 \mu \mathrm{L} \quad 3.0 \quad \mathrm{mM}$ solution of a standard (1,4-dibromo-2,5-difluorobenzene) and $500 \mu \mathrm{L}$ of $1.5 \mathrm{mM}$ lithium alkynide ${ }^{\mathrm{C} 2} \mathrm{ArC} \equiv \mathrm{CLi}$ stock solution in THF $(8.0 \mathrm{~mL})$ were added to the reaction vial. The reaction mixture was stirred until the violet color of $\mathbf{3}$ decolorized. The resultant mixture was then filtered to remove any insoluble material and subsequently was analyzed by GC-MS without any further workup. The ratios of integrated areas (product/standard) were obtained from GC analysis of the sample (Figure S12). A response factor obtained from calibration curve was applied to determine the GC yields based on 1.0 equivalent of standard (1,4-dibromo-2,5-difluorobenzene) added to each reaction. The yield was determined to be $71 \%$ for the formation of the homocoupled product $5\left({ }^{\mathrm{Cl} 2} \mathrm{ArC} \equiv \mathrm{C}-\mathrm{C} \equiv \mathrm{CAr}{ }^{\mathrm{Cl} 2}\right)$. Notably, these reaction occur with concomitant formation of $\left[{ }^{i} \mathrm{Pr}_{2} \mathrm{NN}\right] \mathrm{Cu}\left(\mathrm{C}_{6} \mathrm{D}_{6}\right)\left(\mathrm{C}_{6} \mathrm{D}_{6}\right.$ is the NMR co-solvent) as well as $\left.\left\{{ }^{i} \mathrm{Pr}_{2} \mathrm{NN}\right] \mathrm{Cu}(\mathrm{C} \equiv \mathrm{CR})\right\}^{-}$. The latter could decay to $\left[{ }^{i} \mathrm{Pr}_{2} \mathrm{NN}\right] \mathrm{Li}\left(\mathrm{THF}-d_{8}\right)+\mathrm{Cu}-\mathrm{C} \equiv \mathrm{CR}$ as confirmed by ${ }^{1} \mathrm{H}$ NMR independently. For instance, reaction of 3 with ${ }^{\mathrm{C} 12} \mathrm{ArC} \equiv \mathrm{CLi}$ alkynide results in the formation of $\left[{ }^{i} \mathrm{Pr}_{2} \mathrm{NN}\right] \mathrm{Cu}\left(\mathrm{C}_{6} \mathrm{D}_{6}\right)$, 
$\left[{ }^{i} \mathrm{Pr}_{2} \mathrm{NN}\right] \mathrm{Li}\left(\mathrm{THF}-d_{8}\right),\left\{\left[{ }^{i} \mathrm{Pr}_{2} \mathrm{NN}\right] \mathrm{Cu}\left(\mathrm{C} \equiv \mathrm{CAr}^{\mathrm{Cl} 2}\right)\right\}^{-}$and ${ }^{\mathrm{Cl} 2} \mathrm{ArC} \equiv \mathrm{C}-\mathrm{C} \equiv \mathrm{CAr}{ }^{\mathrm{Cl} 2}$ as shown in its ${ }^{1}$ H NMR (Figure S13).

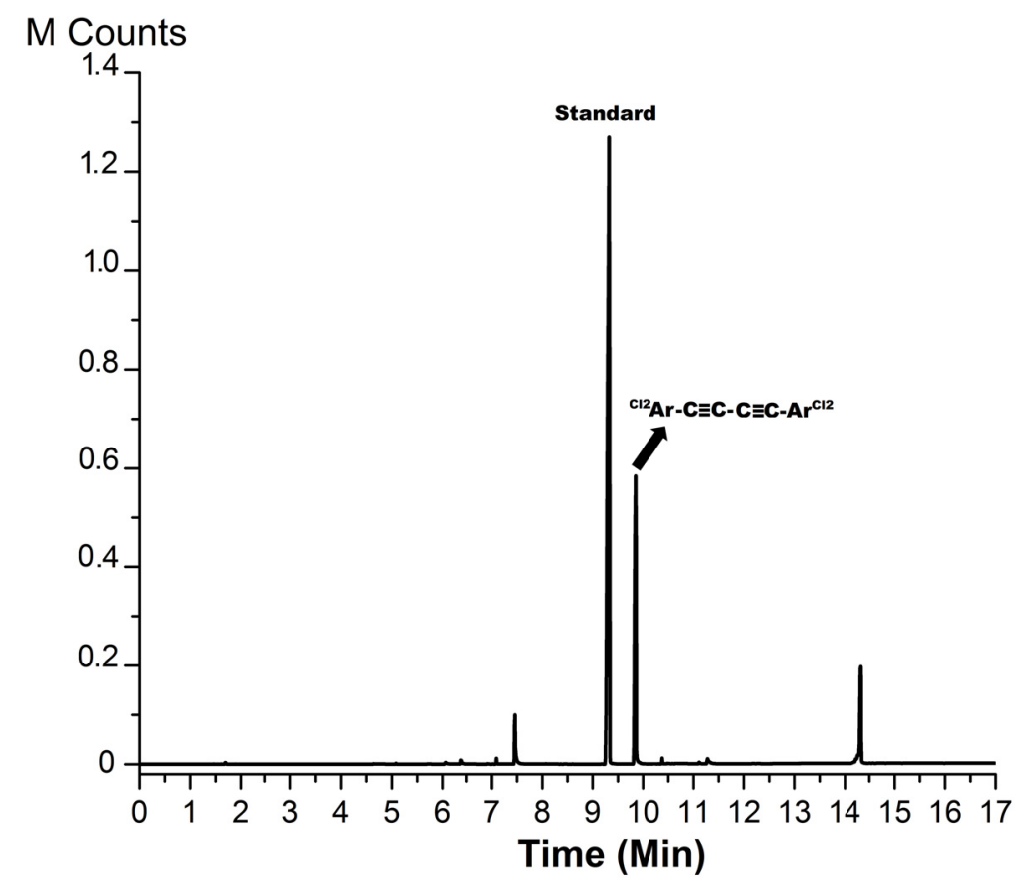

Figure S12. GC-MS (EI mode) profile of the crude reaction mixture obtained upon reaction of ${ }^{\mathrm{Cl} 2} \mathrm{ArC} \equiv \mathrm{CLi}$ with $\left[{ }^{i} \mathrm{Pr}_{2} \mathrm{NN}\right] \mathrm{Cu}-\mathrm{C} \equiv \mathrm{CAr}^{\mathrm{Cl} 2}$ (3) $\quad(1.0 \mathrm{~mL}, 1.5 \mathrm{mM})$ and 1,4-dibromo-2,5-difluorobenzene (as internal standard) in cold THF. Top: 1,3-diyne ${ }^{\mathrm{C} 2} \mathrm{ArC} \equiv \mathrm{C}-\mathrm{C} \equiv \mathrm{CAr}{ }^{\mathrm{C} 12}$ (5) (retention time: 9.777 min) and 1,4-dibromo-2,5-difluorobenzene (retention time: $9.211 \mathrm{~min}$ ). For MS-EI mode spectrum of $1,3-$ diyne ${ }^{\mathrm{Cl} 2} \mathrm{ArC} \equiv \mathrm{C}-\mathrm{C} \equiv \mathrm{CAr}^{\mathrm{Cl} 2}$ (5) see Figure $\mathrm{S} 10$. 


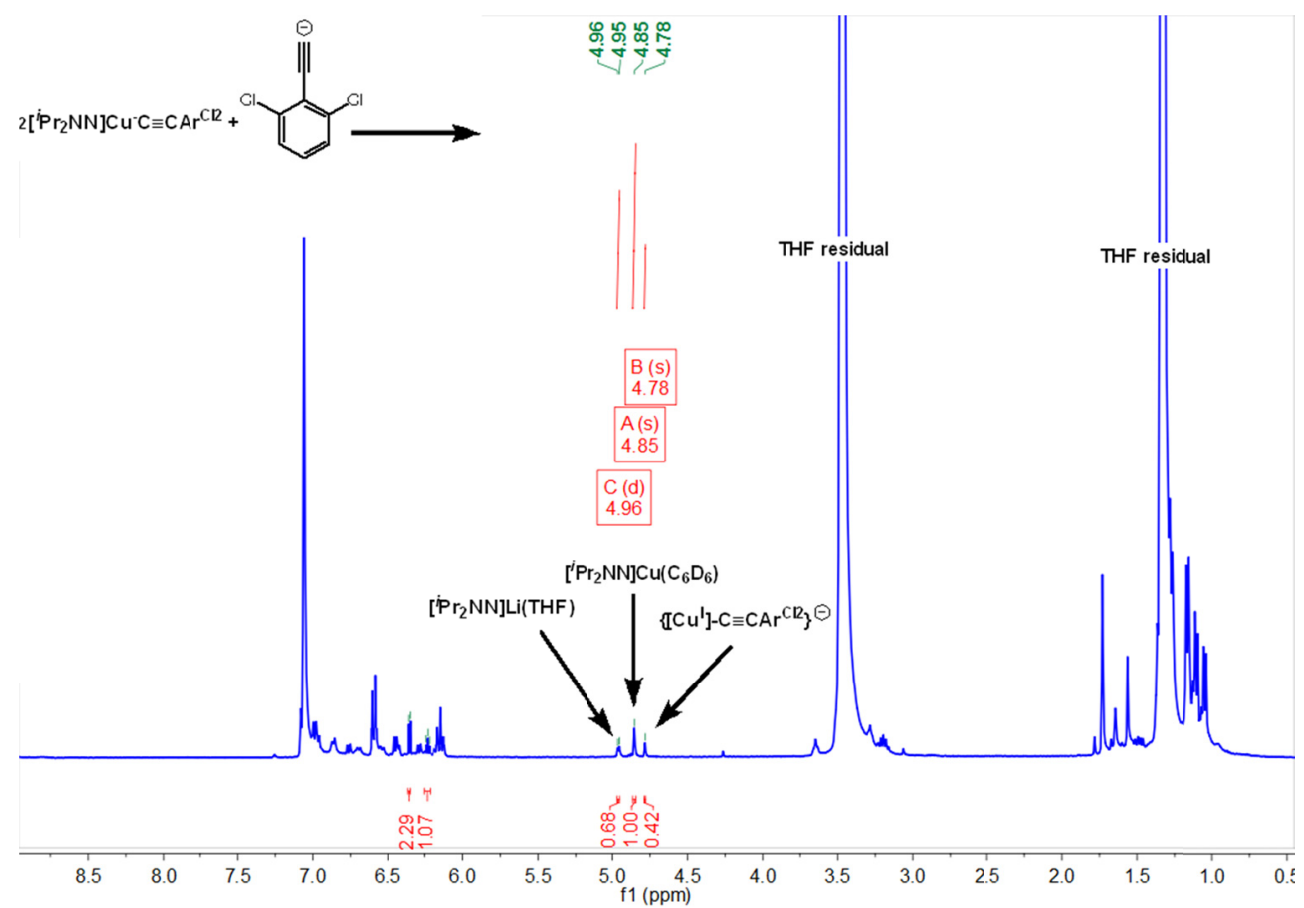

Figure S13. ${ }^{1} \mathrm{H}$ NMR spectrum $\left(400 \mathrm{MHz}, 298 \mathrm{~K}, \mathrm{C}_{6} \mathrm{D}_{6}\right)$ of the crude solution obtained upon addition of 0.5 equiv. lithium alkynide ${ }^{\mathrm{Cl} 2} \mathrm{ArC} \equiv \mathrm{CLi}$ in THF to 1.0 equivalent 3 in $\mathrm{C}_{6} \mathrm{D}_{6}$. Formation and yields of ${ }^{\mathrm{Cl} 2} \mathrm{ArC} \equiv \mathrm{C}-\mathrm{C} \equiv \mathrm{CAr}{ }^{\mathrm{Cl} 2}$ (5) was confirmed by $\mathrm{GC}-\mathrm{MS}$ as shown in Figures S12. 


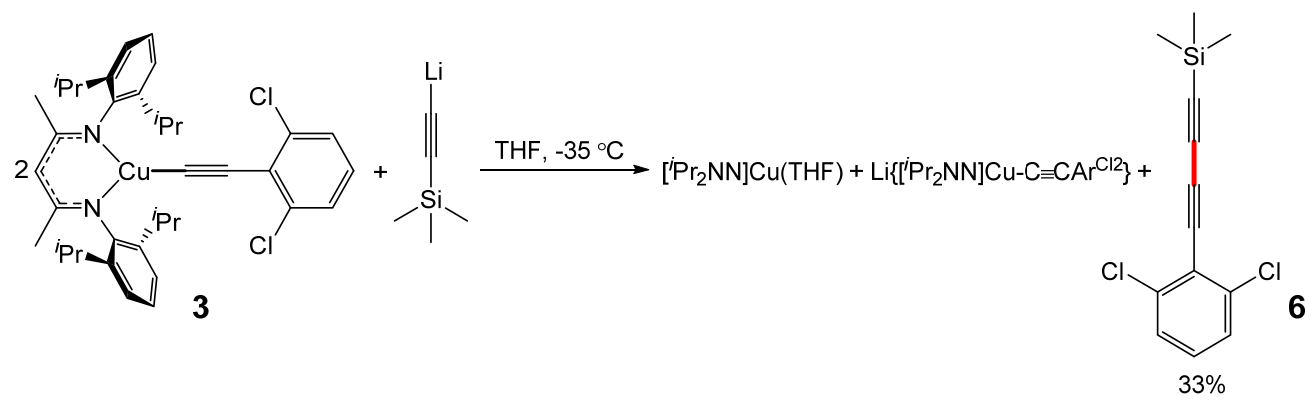

Scheme S7. Reaction of 3 with $\mathrm{Me}_{3} \mathrm{SiC} \equiv \mathrm{CLi}$ anion in cold THF to furnish heterocoupled product $\mathrm{Me}_{3} \mathrm{SiC} \equiv \mathrm{C}-\mathrm{C} \equiv \mathrm{CAr}^{\mathrm{Cl} 2}(\mathbf{6})$.

$1000 \mu \mathrm{L} 1.5 \mathrm{mM}$ of a stock solution of $\mathbf{3}$ was added to a scintillation vial equipped with micro stir bar. $500 \quad \mu \mathrm{L} \quad 3.0 \quad \mathrm{mM}$ solution of standard (1,4-dibromo-2,5-difluorobenzene) and $500 \mu \mathrm{L}$ of $1.5 \mathrm{mM}$ lithium alkynide $\mathrm{Me}_{3} \mathrm{SiC} \equiv \mathrm{CLi}$ stock solution in THF $(5.0 \mathrm{~mL})$ were added to the reaction vial. The reaction mixture was stirred until the violet color of $\mathbf{3}$ decolorized. The resultant mixture was then filtered to remove any insoluble material and subsequently was analyzed by GC-MS without any further workup. The ratios of integrated areas (product/standard) were obtained from GC analysis of the sample (Figure S14). A response factor obtained from calibration curve was applied to determine the GC yields based on 1.0 equivalent of standard (1,4-dibromo-2,5-difluorobenzene) added to each reaction. In case of $\mathrm{Me}_{3} \mathrm{SiC} \equiv \mathrm{CLi}, 36 \%$ of the desired heterocoupled product $\mathrm{Me}_{3} \mathrm{SiC} \equiv \mathrm{C}-\mathrm{C} \equiv \mathrm{CAr}^{\mathrm{Cl} 2}$ was determined along with $33 \%$ of homocoupled product $5\left({ }^{\mathrm{Cl} 2} \mathrm{ArC} \equiv \mathrm{C}-\mathrm{C} \equiv \mathrm{CAr}{ }^{\mathrm{Cl} 2}\right.$ ) and $23 \%$ of homocoupled product $\mathrm{Me}_{3} \mathrm{SiC} \equiv \mathrm{C}-\mathrm{C} \equiv \mathrm{CSiMe}_{3}$. 

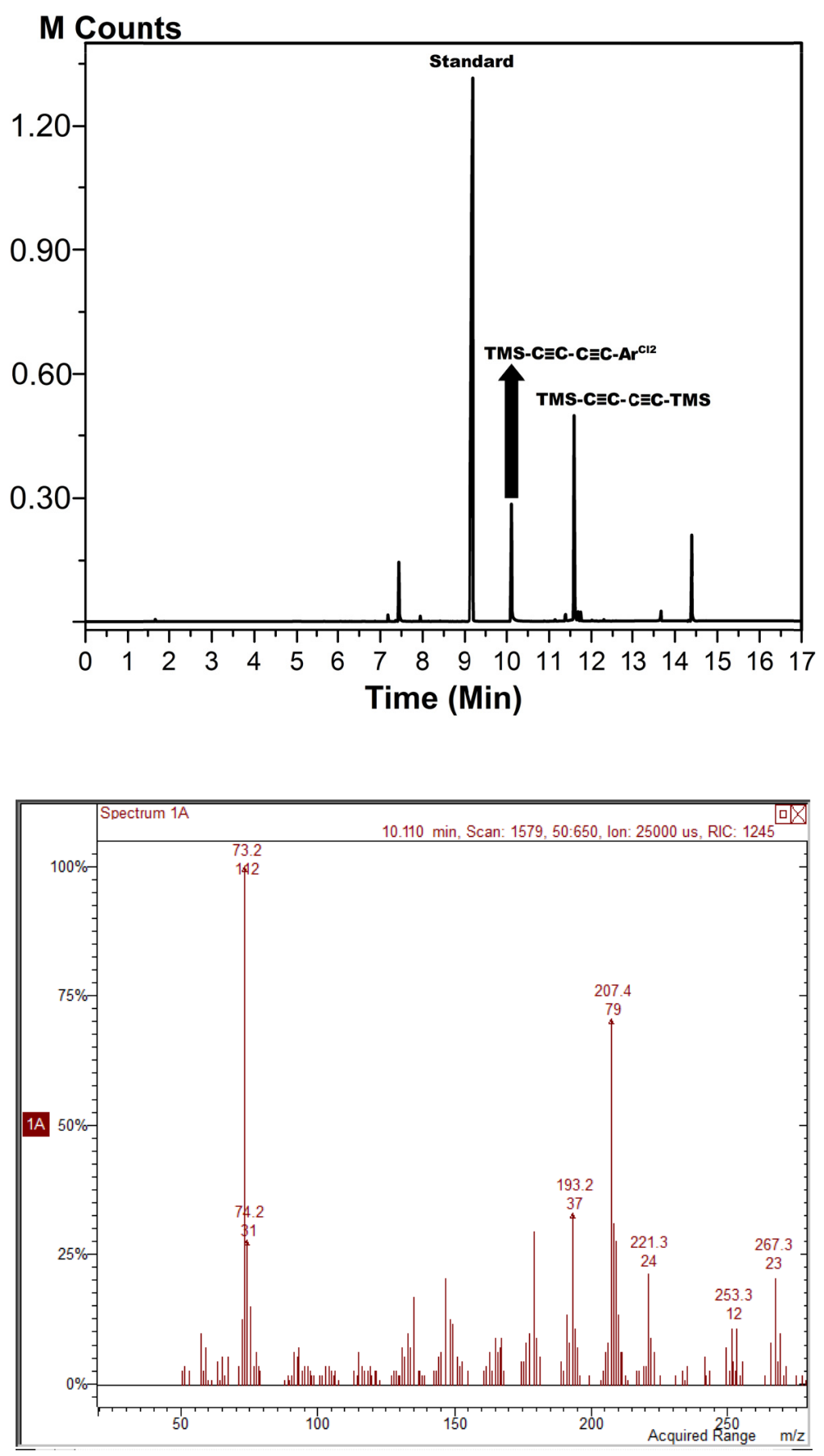

Figure S14. GC-MS (EI mode) profile of the crude reaction mixture obtained upon reaction of $\mathrm{Me}_{3} \mathrm{SiC} \equiv \mathrm{CLi}$ with $\left[{ }^{i} \mathrm{Pr}_{2} \mathrm{NN}\right] \mathrm{Cu}-\mathrm{C} \equiv \mathrm{CAr}^{\mathrm{Cl} 2}$ (3) (1.0 mL, $\left.1.5 \mathrm{mM}\right)$ and 1,4-dibromo-2,5-difluorobenzene (as internal standard) in cold THF. Top: GC-FID plot showing 1,3-diyne $\mathrm{Me}_{3} \mathrm{SiC} \equiv \mathrm{C}-\mathrm{C} \equiv \mathrm{CAr}^{\mathrm{Cl} 2}$ (6) (retention time: $10.110 \mathrm{~min}$ ) and 1,4-dibromo-2,5-difluorobenzene (retention time: $9.190 \mathrm{~min}$ ). Bottom: MS-EI mode spectra of ((2,6-dichlorophenyl)buta-1,3-diyn-1-yl)trimethylsilane (6) (calc. $m / z$ for $\left.\mathrm{C}_{13} \mathrm{H}_{12} \mathrm{Cl}_{2} \mathrm{Si}: 266.01\right)$. 


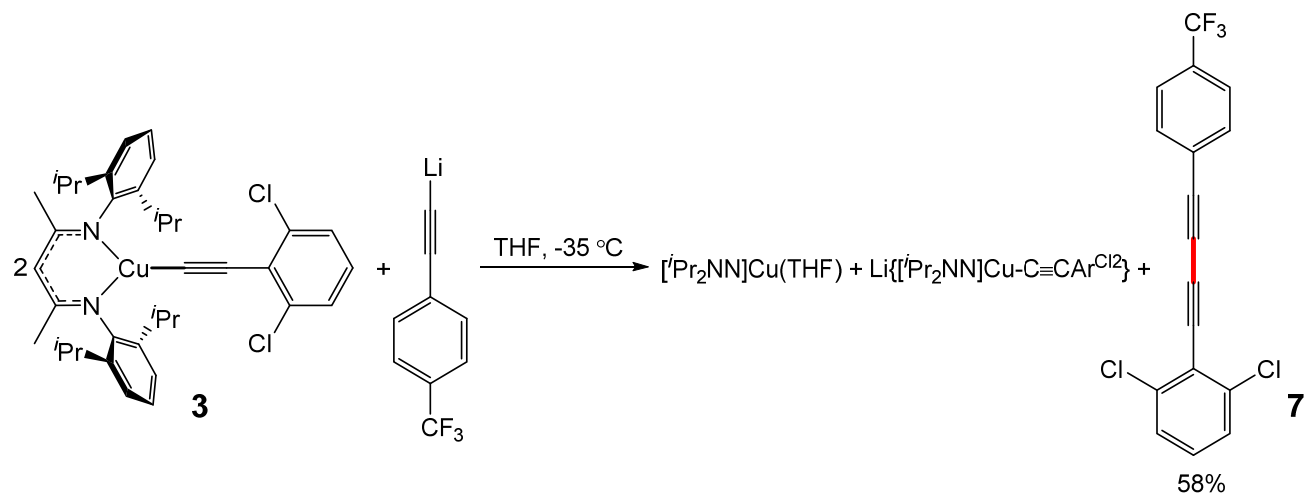

Scheme S8. Reaction of 3 with ${ }^{\mathrm{CF} 3} \mathrm{Ar}-\mathrm{C} \equiv \mathrm{C}^{-}$anion $\left({ }^{\mathrm{CF} 3} \mathrm{Ar}=4-\mathrm{CF}_{3}-\mathrm{C}_{6} \mathrm{H}_{4}\right)$ in cold $\mathrm{THF}$ to generate heterocoupled product ${ }^{\mathrm{CF} 3} \mathrm{ArC} \equiv \mathrm{C}-\mathrm{C}-\mathrm{C} \equiv \mathrm{CAr}^{\mathrm{Cl} 2}$ (7).

$1000 \mu \mathrm{L} 1.5 \mathrm{mM}$ of a stock solution of $\mathbf{3}$ was added to a scintillation vial equipped with micro stir bar. $500 \mu \mathrm{L} \quad 3.0 \quad \mathrm{mM}$ solution of standard (1,4-dibromo-2,5-difluorobenzene) and $500 \mu \mathrm{L}$ of $1.5 \mathrm{mM}$ lithium alkynide ${ }^{\mathrm{CF} 3} \mathrm{Ar}-\mathrm{C} \equiv \mathrm{CLi}$ stock solution in THF $(5.0 \mathrm{~mL})$ were added to the reaction vial. The reaction mixture was stirred until the violet color of $\mathbf{3}$ decolorized. The resultant mixture was then filtered to remove any insoluble material and subsequently was analyzed by ${ }^{19} \mathrm{~F}$ NMR and GC-MS without any further workup. The ratios of integrated areas (product/standard) were obtained from GC analysis of the sample. A response factor obtained from calibration curve was applied to determine the GC yields based on 1.0 equivalent of standard (1,4-dibromo-2,5-difluorobenzene) added to each reaction. For lithium alkynide ${ }^{\mathrm{CF} 3} \mathrm{ArC} \equiv \mathrm{CLi}, \quad 58 \%$ of the desired heterocoupled product ${ }^{\mathrm{CF} 3} \mathrm{ArC} \equiv \mathrm{C}-\mathrm{C}-\mathrm{C} \equiv \mathrm{CAr}{ }^{\mathrm{Cl} 2}$ (7) was determined along with $13 \%$ of homocoupled product 5 $\left({ }^{\mathrm{C} 12} \mathrm{ArC} \equiv \mathrm{C}-\mathrm{C} \equiv \mathrm{CAr}{ }^{\mathrm{C} 12}\right)$ and $17 \%$ of homocoupled product ${ }^{\mathrm{CF} 3} \mathrm{ArC} \equiv \mathrm{C}-\mathrm{C} \equiv \mathrm{CAr}{ }^{\mathrm{CF} 3}$. 

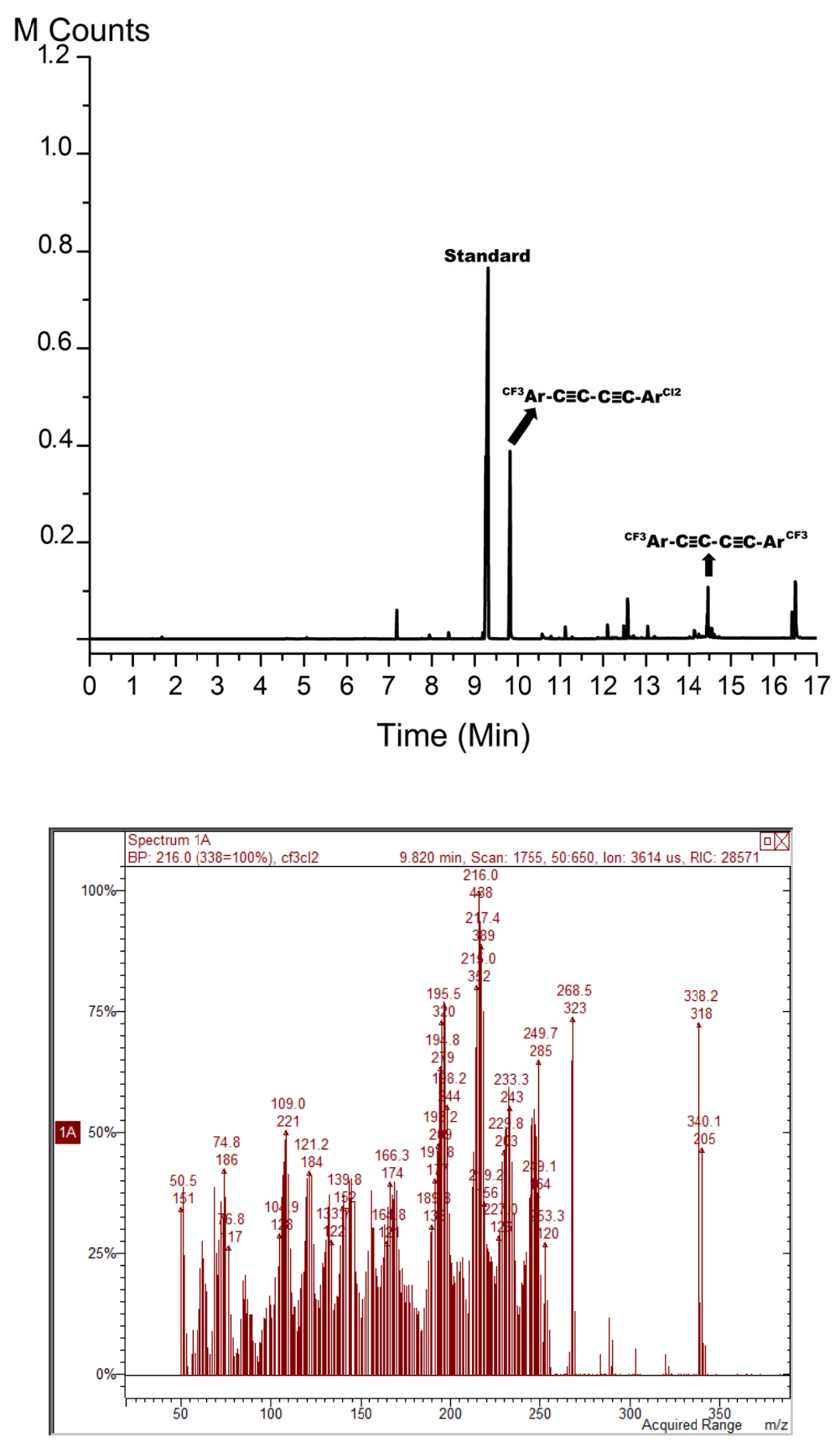

Figure S15. GC-MS (EI mode) profile of crude reaction mixture obtained upon reaction of $\mathrm{CF}_{3} \mathrm{ArC} \equiv \mathrm{CLi}$ with $\quad\left[{ }^{i} \mathrm{Pr}_{2} \mathrm{NN}\right] \mathrm{Cu}-\mathrm{C} \equiv \mathrm{CAr}^{\mathrm{Cl} 2} \quad$ (3) $\quad(1.0 \quad \mathrm{~mL}, \quad 1.5 \quad \mathrm{mM})$ and 1,4-dibromo-2,5-difluorobenzene (as internal standard) in cold THF. Top: GC-FID plot showing 1,3-diyne ${ }^{\mathrm{CF} 3} \mathrm{ArC} \equiv \mathrm{C}-\mathrm{C}-\mathrm{C} \equiv \mathrm{CAr}^{\mathrm{Cl} 2}$ (7) (retention time: $9.820 \mathrm{~min}$ ) and 1,4-dibromo-2,5-difluorobenzene (retention time: $9.208 \mathrm{~min}$ ). Bottom: MS-EI mode spectra of 1,3-dichloro-2-((4-(trifluoromethyl)phenyl)buta-1,3-diyn-1-yl)benzene (7) (calc. $m / z$ for $\mathrm{C}_{13} \mathrm{H}_{7} \mathrm{Cl}_{2} \mathrm{~F}_{3}$ : 337.99). 


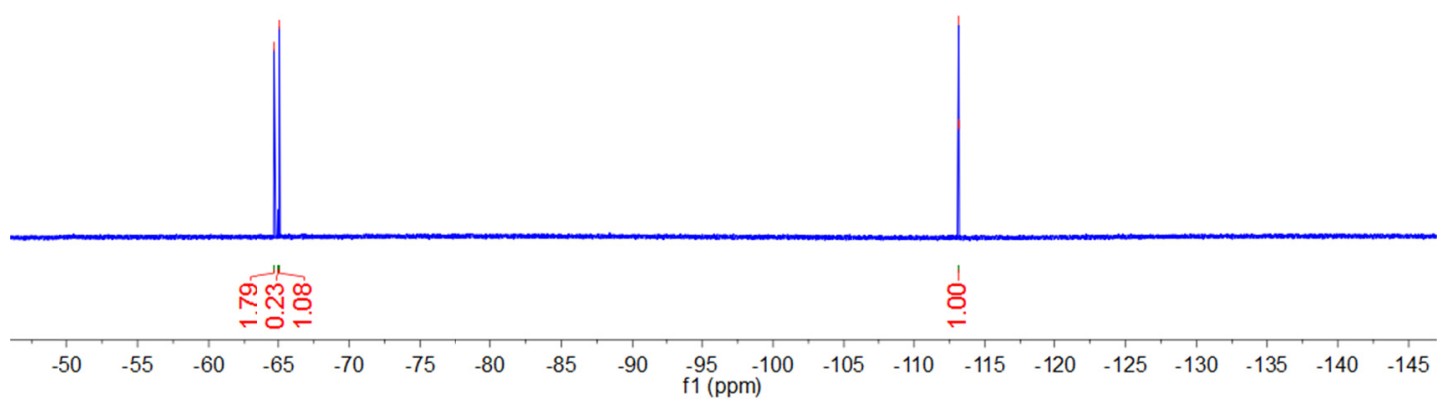

Figure S16. ${ }^{19} \mathrm{~F}$ NMR spectrum $\left(379 \mathrm{MHz}, 298 \mathrm{~K}, \mathrm{THF}-d_{8}\right)$ of the crude reaction mixture obtained upon reaction of ${ }^{\mathrm{CF} 3} \mathrm{ArC} \equiv \mathrm{CLi} \quad(0.5 \mathrm{~mL}, 1.5 \mathrm{mM})$ with $\left[{ }^{i} \mathrm{Pr}_{2} \mathrm{NN}\right] \mathrm{Cu}-\mathrm{C} \equiv \mathrm{CAr}^{\mathrm{Cl} 2}(3)(1.0 \mathrm{~mL}, 1.5 \mathrm{mM})$ and fluorobenzene $(\delta=-113.15 \mathrm{ppm}$ vs. $\mathrm{CFCl}_{3}$ as internal standard, 1.0 equiv. with respect to ${ }^{\mathrm{CF} 3} \mathrm{ArC} \equiv \mathrm{C}^{-}$). The ${ }^{19} \mathrm{~F}$ resonance at $\delta$ $=-64.69 \mathrm{ppm}$ arises from the fluorine atoms on the $\mathrm{CF}_{3}$ group of the product ${ }^{\mathrm{CF} 3} \mathrm{ArC} \equiv \mathrm{C}-\mathrm{C} \equiv \mathrm{CAr}{ }^{\mathrm{Cl} 2}(7)$ and signal at $\delta=-65.06 \mathrm{ppm}$ represents the $\mathrm{CF}_{3}$ groups of the homocoupled product $\left({ }^{\mathrm{CF} 3} \mathrm{ArC} \equiv \mathrm{C}-\mathrm{C} \equiv \mathrm{CAr}{ }^{\mathrm{CF} 3}\right)$. Relative integrations of the ${ }^{19} \mathrm{~F} \mathrm{NMR}$ resonances with respect to internal reference provide $58 \%$ yield for the formation of ${ }^{\mathrm{CF} 3} \mathrm{ArC} \equiv \mathrm{C}-\mathrm{C} \equiv \mathrm{CAr}^{\mathrm{Cl} 2}$ (7) and $16 \%$ for the formation of ${ }^{\mathrm{CF} 3} \mathrm{ArC} \equiv \mathrm{C}-\mathrm{C} \equiv \mathrm{CAr}{ }^{\mathrm{CF} 3}$. These yields were further confirmed by $\mathrm{GC}$ analysis. The signal at $\delta=-64.91 \mathrm{ppm}$ indicates residual of an impurity, presumably unreacted lithium alkynide ${ }^{\mathrm{CF} 3} \mathrm{ArC} \equiv \mathrm{CLi}$ (6.7\%). 
9. Reactivity of $\left[{ }^{i} \mathrm{Pr}_{2} \mathrm{NN}\right] \mathrm{Cu}-\mathrm{C} \equiv \mathrm{CAr}{ }^{\mathrm{Cl} 2}$ with Different Equivalents of (2,6-Dichlorophenyl)ethyn-1-ide.

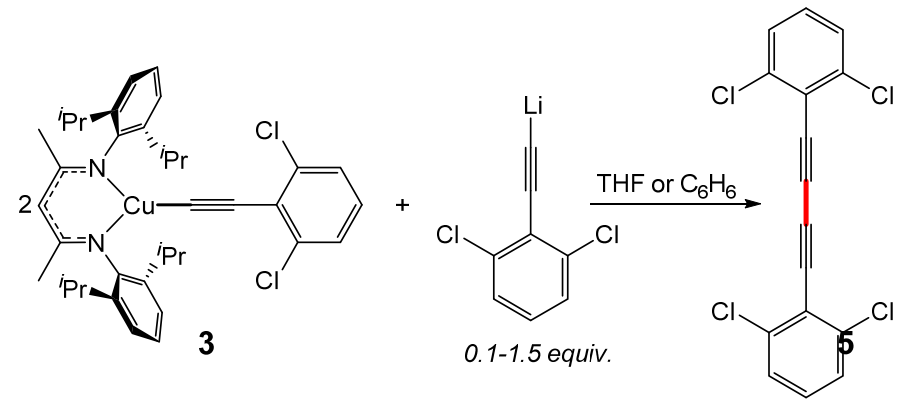

Scheme S9. Reaction of $\left[{ }^{i} \mathrm{Pr}_{2} \mathrm{NN}\right] \mathrm{Cu}-\mathrm{C} \equiv \mathrm{CAr}^{\mathrm{Cl} 2}$ (3) with different equivalents of lithium (2,6-dichlorophenyl)ethyn-1-ide.

Stock solutions of $\left[{ }^{i} \mathrm{Pr}_{2} \mathrm{NN}\right] \mathrm{Cu}-\mathrm{C} \equiv \mathrm{CAr}^{\mathrm{Cl} 2}$ (3), 1,4-dibromo-2,5-difluorobenzene (standard), and lithium (2,6-dichlorophenyl)ethyn-1-ide ( $\left.{ }^{\mathrm{C} 12} \mathrm{ArC} \equiv \mathrm{CLi}\right)$ were prepared by dissolving calculated amounts of individual compounds in specific amounts of $\mathrm{C}_{6} \mathrm{H}_{6}$ or THF as tabulated below:

Table S1. Preparation of stock solutions for reactivity analysis of $\mathbf{3}^{\mathrm{Me}}$.

\begin{tabular}{cccccc}
\hline Compound & Weight $(\mathrm{g})$ & mmol & Solvent & Volume $(\mathrm{mL})$ & $\begin{array}{c}\text { Concentration } \\
(\mathrm{mM})\end{array}$ \\
\hline $\mathbf{3}$ & 0.050 & 0.077 & $\mathrm{C}_{6} \mathrm{H}_{6}$ & 19.480 & 1.5 \\
Standard & 0.018 & 0.065 & $\mathrm{C}_{6} \mathrm{H}_{6}$ & 21.577 & 3.0 \\
${ }^{\mathrm{Cl} 2} \mathrm{Ar}=\mathrm{C}-\mathrm{C}^{-}$ & 0.012 & 0.068 & $\mathrm{THF}$ & 22.124 & 1.5 \\
\hline
\end{tabular}

Independent reactions between 3 and different equivalents $(0.1,0.2,0.3,0.4,0.5$, $0.6,0.7,0.8,0.9,1.0,1.5$, and 2.0) of lithium alkynide were carried out and analyzed by $\mathrm{GC}-\mathrm{MS}$ for determining the yield of desired product ${ }^{\mathrm{Cl} 2} \mathrm{ArC} \equiv \mathrm{C}-\mathrm{C} \equiv \mathrm{CAr}{ }^{\mathrm{Cl} 2}$.

$1000 \mu \mathrm{L}$ of a $1.5 \mathrm{mM}$ stock solution of $\mathbf{3}$ was added to each vial equipped with micro stir bar. $500 \mu \mathrm{L} 3.0 \mathrm{mM}$ solution of standard (1,4-dibromo-2,5-difluorobenzene) and calculated amounts of $1.5 \mathrm{mM}$ lithium alkynide stock solution were added to each vial. The reaction mixtures were stirred until the violet color of $\mathbf{3}$ bleached. The resultant mixture was then filtered to remove any insoluble material and subsequently was analyzed by GC-MS without any additional workup. The ratios of integrated areas 
(product/standard) were obtained from GC analysis of each sample. A response factor obtained from calibration curve was applied to determine the GC yields based on 1.0 equivalent of standard (1,4-dibromo-2,5-difluorobenzene) added to each reaction.

Table S2. Yields of ${ }^{\mathrm{Cl} 2} \mathrm{Ar} \equiv \mathrm{C}-\mathrm{C} \equiv \mathrm{CAr}{ }^{\mathrm{Cl} 2}$ (5) from the reaction of $\left[{ }^{i} \mathrm{Pr}_{2} \mathrm{NN}\right] \mathrm{Cu}-\mathrm{C} \equiv \mathrm{CAr}{ }^{\mathrm{Cl}}$ (3) with different equivalents of lithium alkynide ${ }^{\mathrm{Cl} 2} \mathrm{Ar}=\mathrm{C}-\mathrm{CLi}$.

\begin{tabular}{cc}
\hline $\begin{array}{c}\text { Equivalents of } \\
{ }_{\mathrm{Cl}} \mathrm{Ar}=\mathrm{C}-\mathrm{CLi}\end{array}$ & $\begin{array}{c}\text { \% Yield }{ }^{\mathrm{a}} \text { of } \\
\text { Glaser product } \\
\mathbf{5}\end{array}$ \\
\hline 0.1 & 13.1 \\
0.2 & 21.3 \\
0.3 & 44.0 \\
0.4 & 55.7 \\
0.5 & 71.2 \\
0.6 & 69.4 \\
0.7 & 68.1 \\
0.8 & 66.6 \\
0.9 & 69.5 \\
1.0 & 71.5 \\
1.5 & 74.3 \\
\hline
\end{tabular}

${ }^{a}$ Yields are based on $\mathbf{3}$ as limiting reagent and a 2:1 stoichiometry between $\mathbf{3}$ and lithium alkynide.

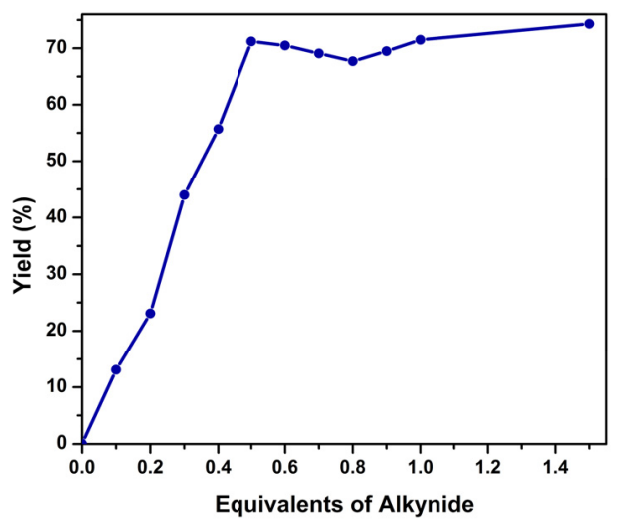

Figure S17. Plot showing the variation in the \%yields of ${ }^{\mathrm{Cl} 2} \mathrm{ArC} \equiv \mathrm{C}-\mathrm{C} \equiv \mathrm{CAr}{ }^{\mathrm{Cl} 2}(\mathbf{5})$ as a function of different equivalents of ${ }^{\mathrm{Cl} 2} \mathrm{ArC} \equiv \mathrm{C}-\mathrm{Li}$ nucleophile added. The graph indicates that the maximum yield of $71.1 \%$ has been obtained from the reaction between 1.0 equiv. 3 and 0.5 equiv. lithium alkynide. 


\section{Reactivity of $\left[{ }^{i} \operatorname{Pr}_{2} \mathrm{NN}\right] \mathrm{Cu}-\mathrm{C} \equiv \mathrm{CAr}^{\mathrm{Cl} 2}$ (3) with Phenyllithium}

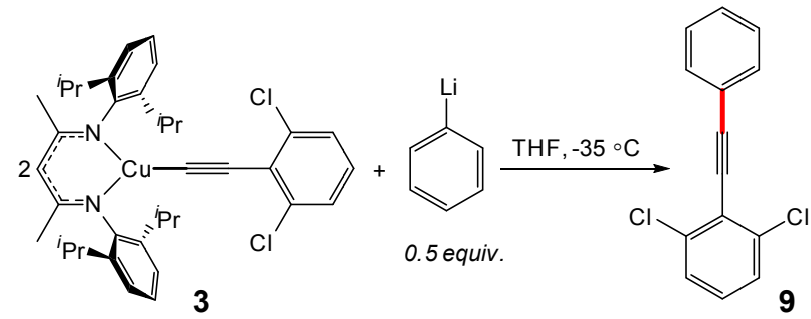

Scheme S10. Reaction of 1.0 equiv. $\left[{ }^{i} \mathrm{Pr}_{2} \mathrm{NN}\right] \mathrm{Cu}-\mathrm{C} \equiv \mathrm{CAr}{ }^{\mathrm{Cl} 2}(3)$ with 0.5 of phenyllithium in THF.

$1000 \mu \mathrm{L}$ of a $2.0 \mathrm{mM}$ stock solution of $\mathbf{3}$ was added to a vial equipped with a micro stir bar. $500 \mu \mathrm{L} 4.0 \mathrm{mM}$ solution of standard (4,4'-di-tert-butylbiphenyl) and $1000 \mu \mathrm{L}$ of a $1.0 \mathrm{mM}$ solution of phenyllithium in THF were added to precooled $\left(-35^{\circ} \mathrm{C}\right)$ vial containing 3 and the internal standard. The reaction mixture was then placed in a $-35^{\circ} \mathrm{C}$ freezer inside a glove box until the intense violet color of $\mathbf{3}$ bleached ( $<1 \mathrm{~min})$. The resultant solution was then analyzed by $\mathrm{GC}-\mathrm{MS}$ without any additional workup. The ratios of integrated areas (product/standard) were obtained from GC analysis of the sample. A response factor obtained from calibration curve was applied to determine the GC yields based on 1.0 equivalent of standard added to the reaction. The yield of 9 independent reactions were averaged and calculated to be $62 \%$ along with $12 \%$ of homocoupled product biphenyl confirmed via GC-MS analysis.

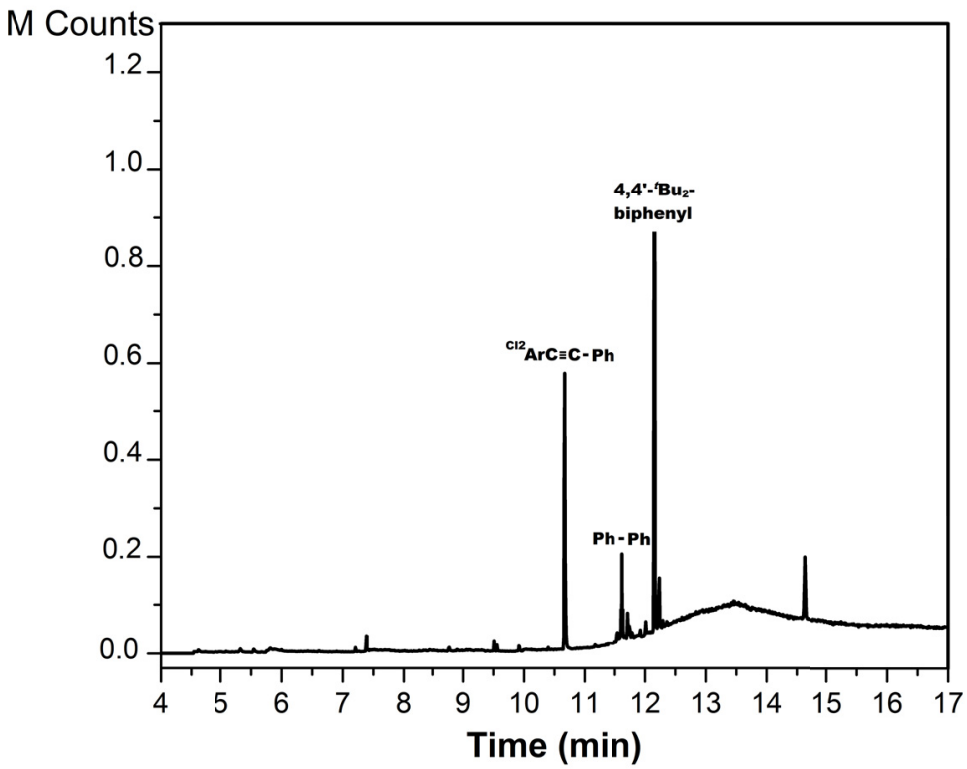




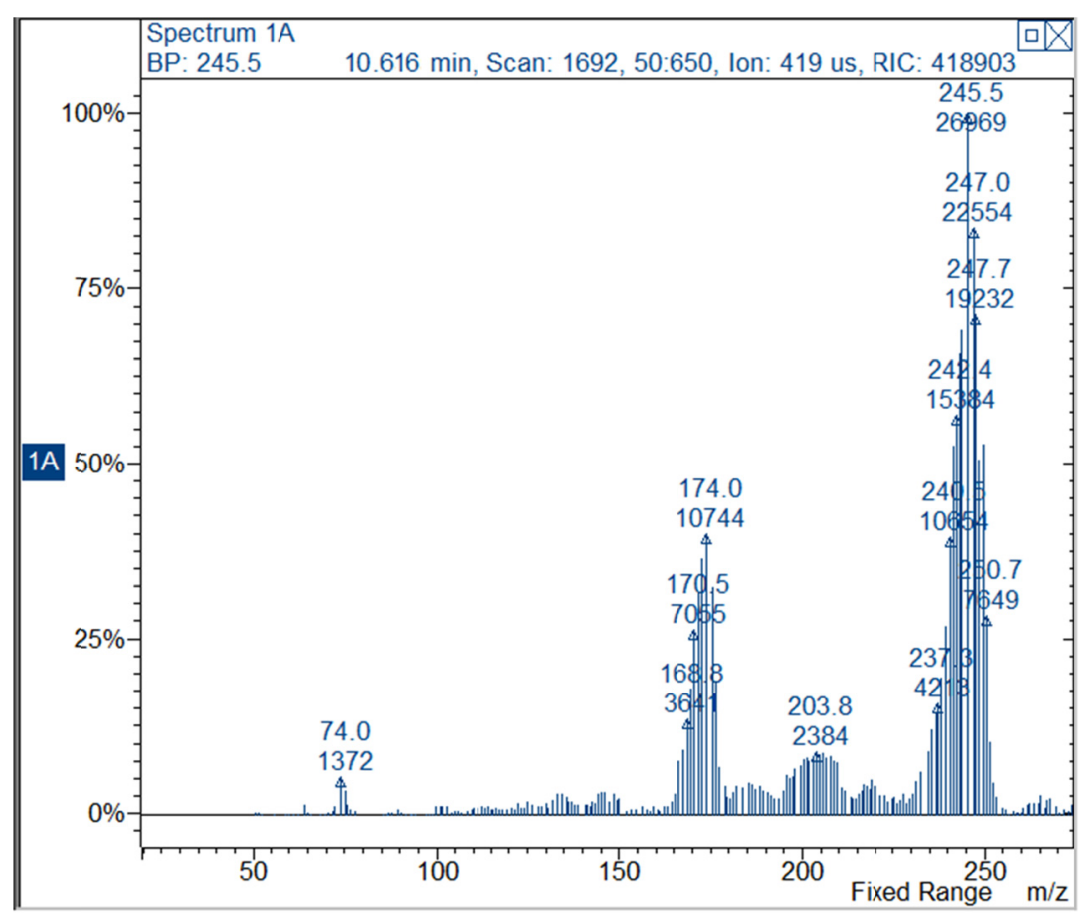

Figure S18. GC-MS (EI mode) profile of the crude reaction mixture obtained upon addition of 0.5 equivalent of phenyllithium ( $\mathrm{PhLi}$ ) to a solution consisting 1.0 equiv. 3 and 1.0 equiv. 4,4'-di-tert-butylbiphenyl (as internal standard) in cold THF. Top: GC-FID plot showing ${ }^{\mathrm{Cl} 2} \mathrm{ArC} \equiv \mathrm{C}-\mathrm{Ph}$ (9) (retention time: $10.616 \mathrm{~min}$ ), biphenyl (retention time: 11.661), and 4,4'-di-tert-butylbiphenyl (retention time: $12.136 \mathrm{~min}$ ). The unassigned peaks correspond ligand residue. Bottom: MS-EI mode spectra of ${ }^{\mathrm{Cl} 2} \mathrm{ArC} \equiv \mathrm{C}-\mathrm{Ph}(\mathbf{9})$ (calc. $\mathrm{m} / \mathrm{z}$ for $\mathrm{C}_{16} \mathrm{H}_{6} \mathrm{Cl}_{4}$ : 246.00). 


\section{Trityl Radical Capture by $\left[{ }^{i} \mathrm{Pr}_{2} \mathrm{NN}_{\mathrm{F}}\right] \mathrm{Cu}-\mathrm{C} \equiv \mathrm{CAr}^{\mathrm{Cl} 2}(3)$.}
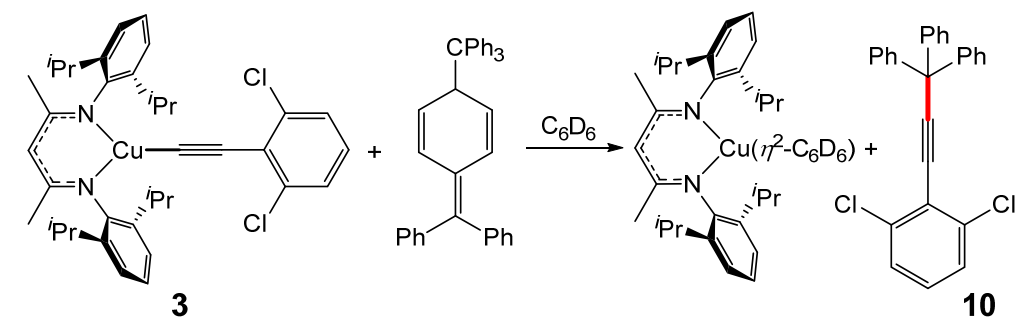

Scheme S11. Trityl radical capture by $\left[{ }^{i} \mathrm{Pr}_{2} \mathrm{NN}\right] \mathrm{Cu}-\mathrm{C} \equiv \mathrm{CAr}^{\mathrm{Cl} 2}(\mathbf{3})$.

The synthesis of the Gomberg's dimer was adapted from a literature procedure. ${ }^{11}$ To a slurry of activated $\mathrm{Zn}$ powder $(250.0 \mathrm{mg}, 3.825 \mathrm{mmol})$ in ca. $30 \mathrm{~mL}$ pentane, $\mathrm{Ph}_{3} \mathrm{CCl}(750.0 \mathrm{mg}, 1.076 \mathrm{mmol})$ was added. Then acetonitrile (ca. $\left.5 \mathrm{~mL}\right)$ was added to form a biphasic mixture and the reaction mixture was then shaken vigorously for $5 \mathrm{~min}$. The pentane layer was decanted and filtered through a pad of Celite. The filtrate was concentrated under reduced pressure and the concentrated solution was set inside a freezer to crystallize at $-35{ }^{\circ} \mathrm{C}$. Yellow solution turned into a very faint yellow color as crystals formed. Pure Gomberg's dimer was formed as $\left(\mathrm{Ph}_{3} \mathrm{C}\right)_{2} \bullet$ pentane $(277 \mathrm{mg}, 0.495$ mmol, $18 \%$ yield). Spectroscopic data were consistent with those reported in the literature. ${ }^{11}$

A stock solution of $\left[{ }^{i} \mathrm{Pr}_{2} \mathrm{NN}\right] \mathrm{Cu}-\mathrm{C} \equiv \mathrm{CAr}{ }^{\mathrm{Cl} 2}\left(0.100 \mathrm{mg}\right.$ of (3) in $3.0 \mathrm{mLC}_{6} \mathrm{D}_{6}$; $0.0512 \mathrm{M}$ ) was used in this experiment. Three vials were charged with $1.0 \mathrm{~mL}$ of the stock solution of 3 and to these vials were added $1.0 \mathrm{~mL}$ of $0.0512 \mathrm{M}$ solution of Gomberg's dimer in $\mathrm{C}_{6} \mathrm{D}_{6}$. The reaction mixture in each vial was stirred at RT until the violet color of $\mathbf{3}$ was discharged. At this point an aliquot was taken from each vial and the yield of the $\mathrm{Ph}_{3} \mathrm{C}-\mathrm{C} \equiv \mathrm{CAr}^{\mathrm{Cl} 2}$ (10) product was determined via $\mathrm{GC}-\mathrm{MS}$. An average yield of $64 \% \pm 2 \%$ was determined. The same experiment was also monitored via ${ }^{1} \mathrm{H}$ NMR and the formation of $\left[{ }^{i} \mathrm{Pr}_{2} \mathrm{NN}\right] \mathrm{Cu}\left(\eta^{2}-\mathrm{C}_{6} \mathrm{D}_{6}\right)$ was confirmed in $78 \% \pm 3 \%$ yield. 


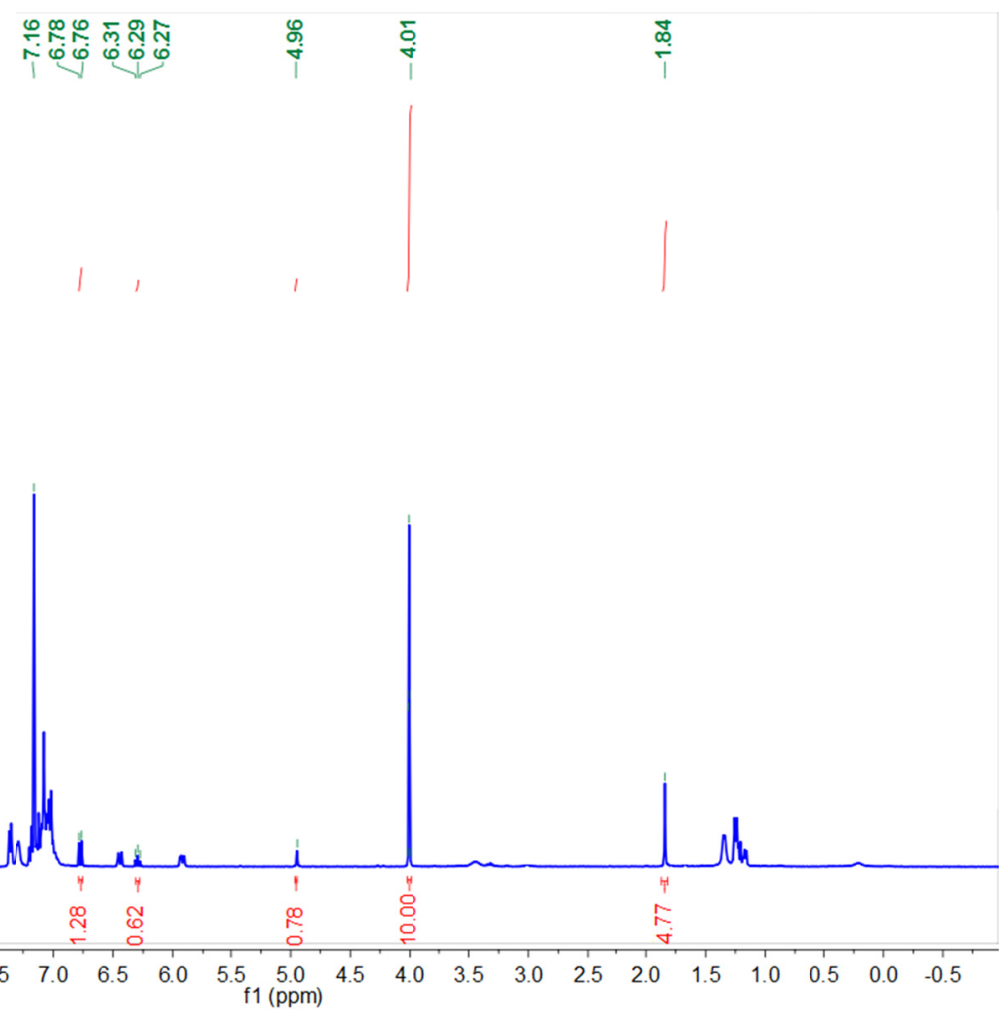

Figure S19. ${ }^{1} \mathrm{H}$ NMR spectrum $\left(400 \mathrm{MHz}, 298 \mathrm{~K}, \mathrm{C}_{6} \mathrm{D}_{6}\right)$ of the crude solution obtained upon addition of 1.0 equiv. of Gomberg's dimer to a solution consisting equimolar amounts of 3 and ferrocene as internal standard in $\mathrm{C}_{6} \mathrm{D}_{6} \cdot{ }^{1} \mathrm{H}$ NMR resonances at $\delta 4.96$ ppm (C-H backbone of $\beta$-diketiminate), $\delta 6.29$ ppm (triplet; $p-\mathrm{Ar}^{\mathrm{Cl} 2}-\mathrm{H}$ ) and $\delta 6.77 \mathrm{ppm}$ (doublet; $m-\mathrm{Ar}-\mathrm{H}$ ) were used to calculate the yield against the signal at $\delta 4.01 \mathrm{ppm}$ (singlet; ferrocene). 


\section{Procedures for Catalytic Alkylation of Terminal Alkynes Catalyzed by $\left[\mathrm{Cl}_{2} \mathrm{NN}\right] \mathrm{Cu}$.}

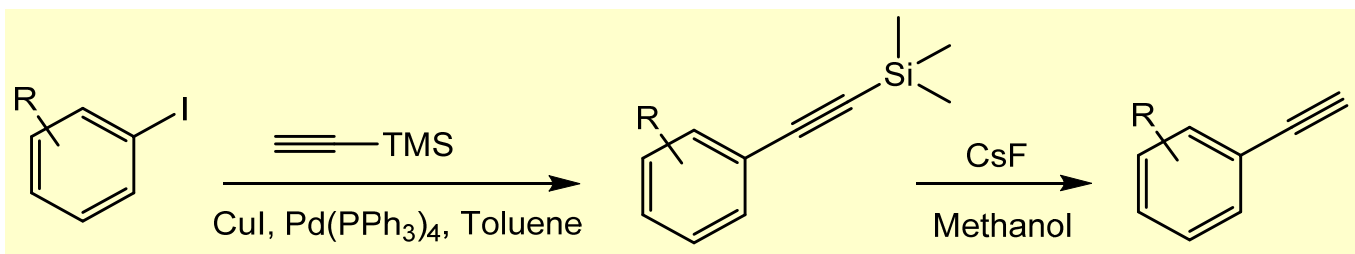

Scheme S12. Synthesis of terminal alkynes used in this study.

\section{Synthesis of terminal alkynes}

Based upon a literature procedure ${ }^{2}$, to a degassed mixture of copper (I) iodide ( $96 \mathrm{mg}$, $0.5 \mathrm{mmol})$, and $\mathrm{Pd}\left(\mathrm{PPh}_{3}\right)_{4}(0.58 \mathrm{~g}, 0.5 \mathrm{mmol})$, dry toluene $(40 \mathrm{~mL})$, diisopropylamine $(4.0 \mathrm{~mL})$ and iodoarene $(10.0 \mathrm{mmol})$ were added. The reaction mixture was stirred at room temperature for $15 \mathrm{~min}$ before trimethylsilylacetylene $(1.50 \mathrm{~mL}, 10.5 \mathrm{mmol})$ was added. The resulting solution was allowed to stir at $80{ }^{\circ} \mathrm{C}$ overnight. After cooling to room temperature, the reaction mixture was diluted with saturate brine followed by extraction with ethyl acetate for three times. The organic layers were combined, washed with DI water, dried over $\mathrm{MgSO}_{4}$, and concentrated under reduced pressure. The product was purified by flash column chromatography $(100 \%$ petroleum ether) to afford the corresponding trimethylsilyl-protected alkyne product. The trimethylsilyl-protected alkynes were then deprotected upon reaction with excess cesium fluoride in methanol. The crude reaction mixture was then subjected to column chromatography to obtain pure terminal alkyne.

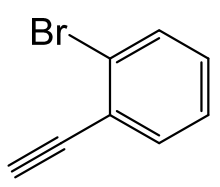

1-bromo-2-ethynylbenzene: 2-bromoiodobenzene (10 mmol) was treated according to the general procedure (Scheme S12) to afford the corresponding TMS-protected alkyne, which was immediately and without further purification treated with CsF (1.5 equiv.) in methanol and left to stir overnight. Upon reaction completion, the mixture was poured into a separating funnel and washed with water and brine. The organic layer was separated, dried over $\mathrm{MgSO}_{4}$ and purified by column chromatography to obtain a yellow oil in $73 \%$ yield. 
${ }^{1} \mathrm{H}$ NMR $\left(399 \mathrm{MHz}, \mathrm{CDCl}_{3}\right) \delta 7.55(\mathrm{~m}, 2 \mathrm{H}), 7.22(\mathrm{~m}, 3 \mathrm{H}), 3.36(\mathrm{~s}, 1 \mathrm{H}),{ }^{13} \mathrm{C}\left\{{ }^{1} \mathrm{H}\right\} \mathrm{NMR}$ $\left(100 \mathrm{MHz}, \mathrm{CDCl}_{3}\right) \delta 134.06,132.43,129.94,126.96,125.54,124.27,81.74$. (1 carbon atom missing or coincident). This compound is known and spectral data matches that in literature. ${ }^{9}$

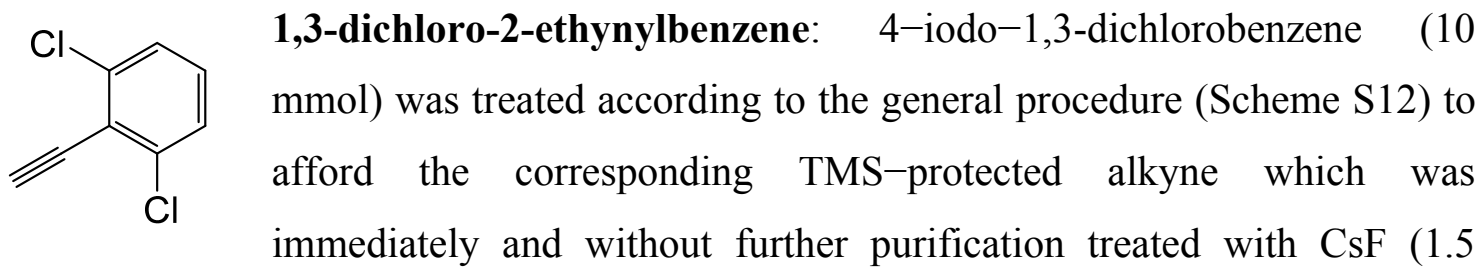
equiv.) in methanol and left to stir overnight. Upon reaction completion, the mixture was poured into a separating funnel and washed with water and brine. The organic layer was separated, dried over $\mathrm{MgSO}_{4}$ and purified by column chromatography to obtain white solid in $66 \%$ yield. ${ }^{1} \mathrm{H}$ NMR $\left(399 \mathrm{MHz}, \mathrm{CDCl}_{3}\right) \delta 7.29(\mathrm{~m}, 2 \mathrm{H}), 7.15$ (dd, $J=8.7,7.5 \mathrm{~Hz}$, $1 \mathrm{H}), 3.63(\mathrm{~s}, 1 \mathrm{H}) .{ }^{13} \mathrm{C}\{1 \mathrm{H}\}$ NMR $(100 \mathrm{MHz}, \mathrm{CDCl} 3) \delta 137.81,129.54,127.52,87.83$. (4 carbon atoms missing or coincident). Spectral data matched literature data. ${ }^{12}$ 


\section{Optimization details for C-H alkynylation}

Yield and selectivity are based on ${ }^{19} \mathrm{~F}$ NMR of the crude reaction mixture using flurobenzene as internal standard ( $\delta=-113.1 \mathrm{ppm})$.

Table S3. Exploring different $\beta$-diketiminato $\mathrm{Cu}$ catalysts for $\mathrm{C}-\mathrm{H}$ alkynylation.

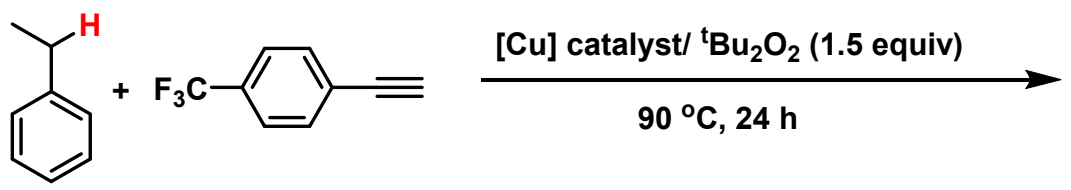

EB

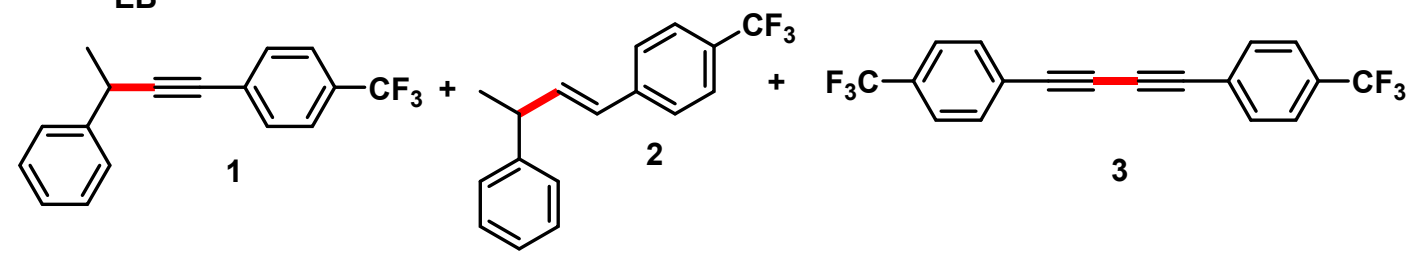

Cu catalyst

\begin{tabular}{|c|c|c|}
\hline $\begin{array}{c}\text { C-H } \\
\text { substrate }\end{array}$ & $\begin{array}{c}\text { Cu catalyst } \\
\text { (1 } \mathrm{mol} \%)\end{array}$ & $\begin{array}{l}\text { yield }(1 / 2 / 3 \\
{ }^{19} \mathrm{~F} \mathrm{NMR}\end{array}$ \\
\hline EB & {$\left[\mathrm{Pr}_{2}{ }_{2} \mathrm{NN}\right] \mathrm{Cu}$} & NR \\
\hline EB & {$\left[{ }^{i} \mathrm{Pr}_{2} \mathrm{NN}\right] \mathrm{Cu}$} & $21 / 9 / 29$ \\
\hline EB & {$\left[\mathrm{Me}_{3} \mathrm{NN}\right] \mathrm{Cu}$} & $26 / 21 / 17$ \\
\hline EB & {$\left[\mathrm{Cl}_{2} \mathrm{NN}\right] \mathrm{Cu}$} & $34 / 17 / 13$ \\
\hline EB & {$\left[\mathrm{Cl}_{2} \mathrm{NN}_{\mathrm{F} 6}\right] \mathrm{Cu}$} & NR \\
\hline
\end{tabular}

$$
\begin{aligned}
& X=\mathrm{CH}_{3}, \mathrm{R}^{1}=\mathrm{CHPh}_{2}, \mathrm{R}^{2}={ }^{t} \mathrm{Bu} ;\left[\mathrm{IPr}_{2}{ }_{2} \mathrm{NN}\right] \mathrm{Cu} \\
& \mathrm{X}=\mathrm{CH}_{3}, \mathrm{R}^{1}={ }^{i} \mathrm{Pr}, \mathrm{R}^{2}=\mathrm{H} ;\left[\mathrm{Pr}_{2} \mathrm{NN}\right] \mathrm{Cu} \\
& \mathrm{X}=\mathrm{CH}_{3}, \mathrm{R}^{1}=\mathrm{Me}, \mathrm{R}^{2}=\mathrm{Me} ;\left[\mathrm{Me}_{3} \mathrm{NN}\right] \mathrm{Cu} \\
& \mathrm{X}=\mathrm{CH}_{3}, \mathrm{R}^{1}=\mathrm{Cl}, \mathrm{R}^{2}=\mathrm{H} ;\left[\mathrm{Cl}_{2} \mathrm{NN}\right] \mathrm{Cu} \\
& X=\mathrm{CF}_{3}, \mathrm{R}^{1}=\mathrm{Cl}, \mathrm{R}^{2}=\mathrm{H} ;\left[\mathrm{Cl}_{2} \mathrm{NN}_{\mathrm{F} 6}\right] \mathrm{Cu}
\end{aligned}
$$


Table S4. Exploring different $\mathrm{Cu}$ catalyst loadings in $\mathrm{C}-\mathrm{H}$ alkynylation.
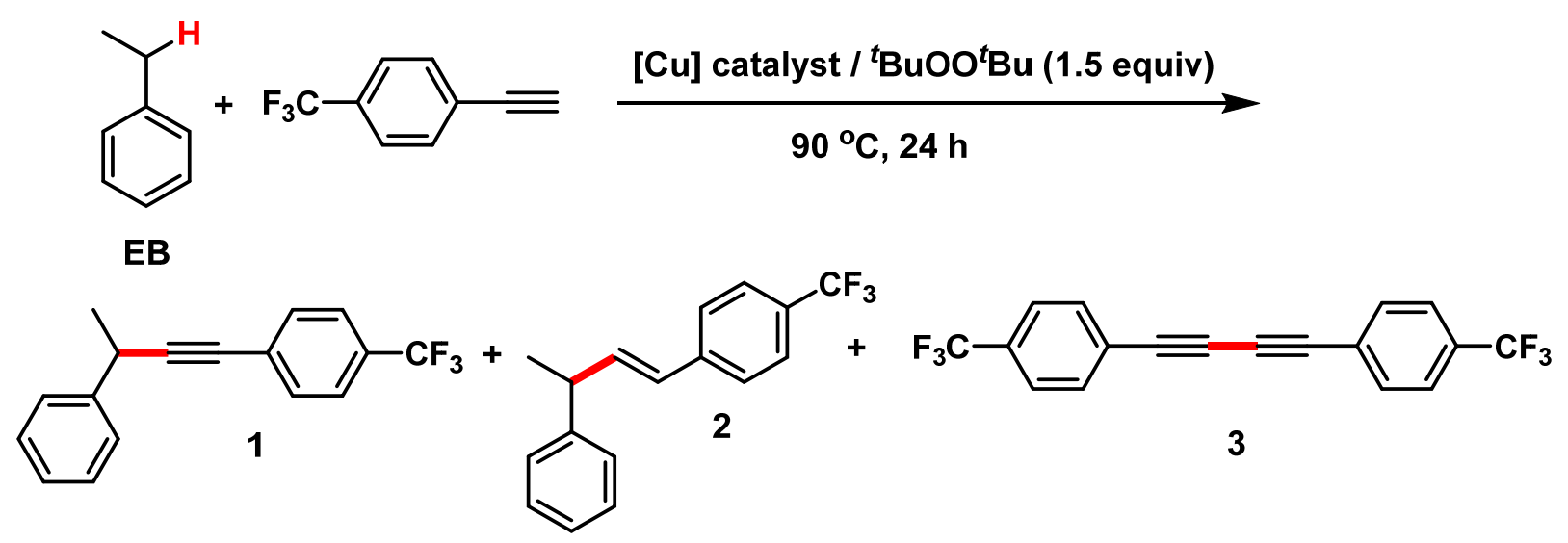

\begin{tabular}{ccc} 
Catalyst loading & & \\
$\begin{array}{c}\text { C-H } \\
\text { substrate }\end{array}$ & {$\left[\mathrm{Cl}_{2} \mathrm{NN}\right] \mathrm{Cu}$} & $\begin{array}{c}\text { yield }(1 / 2 / 3) \% \\
19 \mathrm{~F} \mathrm{NMR}\end{array}$ \\
\hline EB & $5 \mathrm{~mol} \%$ & $1 / 2 / 3: 18 / 11 / 49$ \\
EB & $2 \mathrm{~mol} \%$ & $1 / 2 / 3: 22 / 10 / 24$ \\
EB & $1 \mathrm{~mol} \%$ & $1 / 2 / 3: 34 / 17 / 13$
\end{tabular}


Table S5. Exploring different oxidants for $\mathrm{C}-\mathrm{H}$ alkynylation.
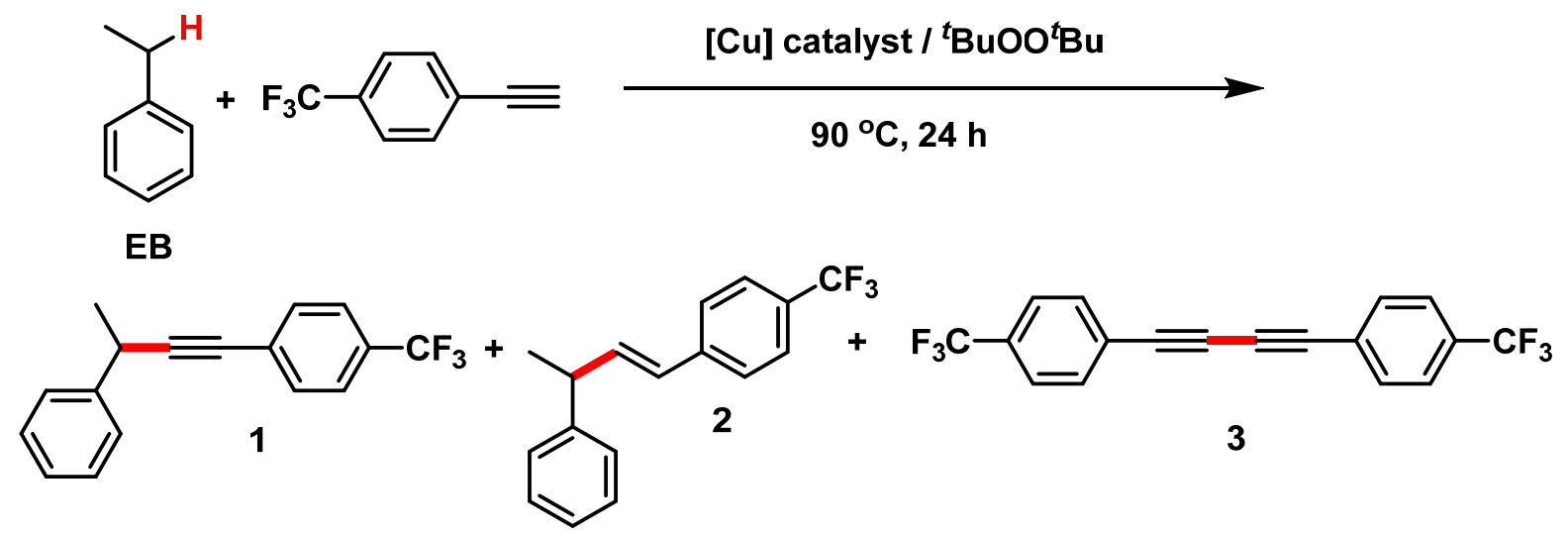

\section{Oxidant}

\begin{tabular}{lcc} 
oxidant & equiv. & $\begin{array}{c}\text { yield }(1 / 2 / 3) \% \\
19 \mathrm{~F} \mathrm{NMR}\end{array}$ \\
\hline PhIO & 1.5 equiv. & $\mathrm{NR}$ \\
$\mathrm{K}_{2} \mathrm{~S}_{2} \mathrm{O}_{7}$ & 1.5 equiv. & $\mathrm{NR}$ \\
Oxone & 1.5 equiv. & $\mathrm{NR}$ \\
${ }^{t} \mathrm{BuOO}{ }^{t} \mathrm{Bu}$ & 1.5 equiv. & $1 / 2 / 3: 34 / 17 / 13$ \\
${ }^{t} \mathrm{BuOO}{ }^{t} \mathrm{Bu}$ & 2.0 equiv. & $1 / 2 / 3: 42 / 18 / 13$
\end{tabular}


Table S6. Exploring different solvents in $\mathrm{C}-\mathrm{H}$ alkynylation.

\begin{tabular}{ccc} 
solvents & & \\
solvent & volume & $\begin{array}{c}\text { yield (1/2/3) \% } \\
19 \mathrm{~F} \mathrm{NMR}\end{array}$ \\
\hline $\mathrm{C}_{6} \mathrm{H}_{6}$ & $0.5 \mathrm{~mL}$ & $58 / 14 / 13$ \\
$\mathrm{C}_{6} \mathrm{H}_{5} \mathrm{~F}$ & $0.5 \mathrm{~mL}$ & $\mathrm{NR}$ \\
$\mathrm{C}_{6} \mathrm{H}_{5} \mathrm{Cl}$ & $0.5 \mathrm{~mL}$ & $64 / 11 / 8$ \\
$\mathrm{C}_{6} \mathrm{H}_{5} \mathrm{Cl}$ & $1.0 \mathrm{~mL}$ & $61 / 14 / 9$
\end{tabular}

(10 equiv. C-H substrate ethylbenzene)

\section{Catalytic C-H alkynylation}
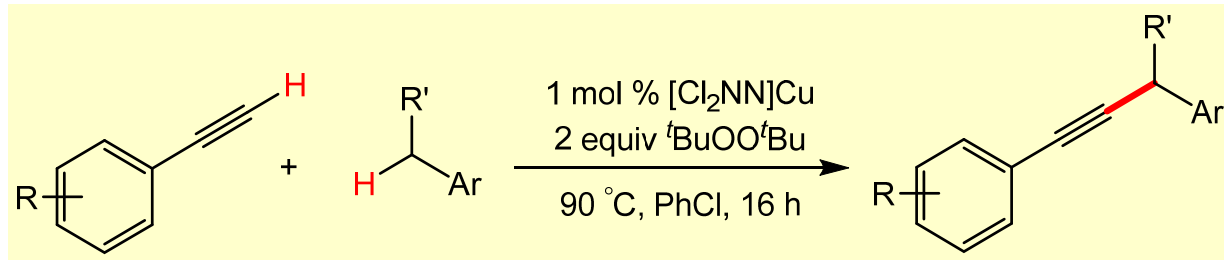

Scheme S13. Catalytic $\mathrm{sp}^{3} \mathrm{C}-\mathrm{H}$ alkynylation of hydrocarbons with alkynes.

In a nitrogen filled glovebox, a pressure vessel charged with a magnetic stir bar, 500 $\mu \mathrm{L}$ chlorobenzene, and $25 \mu \mathrm{L}$ of a stock solution of $\left[\mathrm{Cl}_{2} \mathrm{NN}\right] \mathrm{Cu}\left(\eta^{2}\right.$-benzene $)(2 \mathrm{mmol}$, $0.2 \mathrm{M}$ solution in cholorobenzene). With stirring, 2-chlorophenylacetylene (11) (0.5 mmol, $61 \mu \mathrm{L}$ ) and the $\mathrm{R}-\mathrm{H}$ substrate (10 equiv.) were added successively. After stirring the mixture for $5 \mathrm{~min}, 2$ equiv. ${ }^{t} \mathrm{BuOO}{ }^{t} \mathrm{Bu}(183 \mu \mathrm{L}, 1 \mathrm{mmol})$ was added to give a yellowish colored solution. The pressure vessel was immediately sealed and stirred at $90{ }^{\circ} \mathrm{C}$ for $16 \mathrm{~h}$. After $16 \mathrm{~h}$, the reaction was quenched by exposure to air. The solution was then dried under reduced pressure to yield a dark brown residue. The solution was then dried under reduced pressure and re-suspended in the minimum amount of dichloromethane (DCM) to dissolve the crude product. The corresponding alkylated alkyne product was isolated and purified by flash chromatography or $\mathrm{SiO}_{2}$ preparative thin layer chromatography (9:1 hexanes/ethylacetate). 


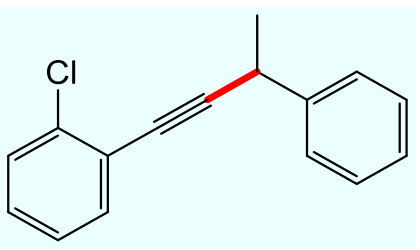

Table 1 entry 12a: 1-chloro-2-(3-phenylbut-1-yn-1yl)benzene. See representative reaction procedure. To a solution of 2-chlorophenylacetylene (11) $(61 \mu \mathrm{L}, 0.5$ $\mathrm{mmol})$ and $\left[\mathrm{Cl}_{2} \mathrm{NN}\right] \mathrm{Cu}\left(\eta^{2}\right.$-benzene $)(25 \mu \mathrm{L}, 1 \mathrm{~mol} \%)$ in chlorobenzene $(0.5 \mathrm{~mL})$ was added ethylbenzene $(1 \mathrm{~mL})$ and ${ }^{t} \mathrm{BuOO}{ }^{t} \mathrm{Bu}(183 \mu \mathrm{L}, 1$ mmol). $\mathrm{SiO}_{2}$ preparative thin layer chromatography afforded the title compound in $81 \%$ $(97 \mathrm{mg})$ yield as a light yellow solid ${ }^{1} \mathrm{H}$ NMR $\left(400 \mathrm{MHz}, \mathrm{CDCl}_{3}\right) \delta 7.52(\mathrm{~m}, 3 \mathrm{H}), 7.42-$ $7.31(\mathrm{~m}, 3 \mathrm{H}), 7.14(\mathrm{~m}, 4 \mathrm{H}), 4.04(\mathrm{q}, J=7.1 \mathrm{~Hz}, 1 \mathrm{H}), 1.62(\mathrm{~d}, J=7.2 \mathrm{~Hz}, 3 \mathrm{H}) .{ }^{13} \mathrm{C}\left\{{ }^{1} \mathrm{H}\right\}$ NMR $\left(100 \mathrm{MHz}, \mathrm{CDCl}_{3}\right) \delta 142.92,134.35,133.24,130.25,129.43,129.13,128.71$, $128.54,126.97,126.68,126.28,123.56,98.11,79.45,32.71,24.46$. HRMS (CI) $\mathrm{m} / \mathrm{z}$ calcd for $\mathrm{C}_{16} \mathrm{H}_{13} \mathrm{Cl}\left(\mathrm{M}^{+}\right)$240.0706, found 240.0709 .

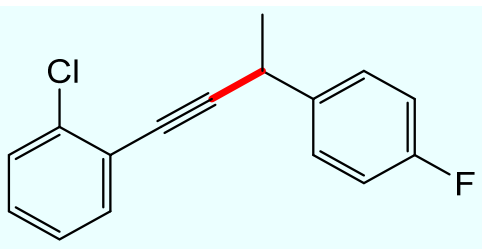

Table 1 entry 12c: 1-chloro-2-(3-(4fluorophenyl)but-1-yn-1-yl)benzene. See representative reaction procedure. To a solution of 2chlorophenylacetylene (11) $(61 \mu \mathrm{L}, 0.5 \mathrm{mmol})$ and $\left[\mathrm{Cl}_{2} \mathrm{NN}\right] \mathrm{Cu}\left(\eta^{2}\right.$-benzene $) \quad(25 \mu \mathrm{L}, \quad 1$ mol \%) in

chlorobenzene $(0.5 \mathrm{~mL})$ was added 4 -fluoro-ethylbenzene $(1 \mathrm{~mL})$ and ${ }^{t} \mathrm{BuOO}{ }^{t} \mathrm{Bu}(183$ $\mu \mathrm{L}, 1 \mathrm{mmol})$. $\mathrm{SiO}_{2}$ preparative thin layer chromatography afforded the title compound in $56 \%$ yield $(72 \mathrm{mg})$ as a yellow solid. ${ }^{1} \mathrm{H} \mathrm{NMR}\left(400 \mathrm{MHz}, \mathrm{CDCl}_{3}\right) \delta 7.41(\mathrm{~m}, 3 \mathrm{H}), 7.29$ (dt, $J=5.6,2.3 \mathrm{~Hz}, 3 \mathrm{H}), 7.02(\mathrm{~m}, 2 \mathrm{H}), 3.96(\mathrm{q}, J=7.2 \mathrm{~Hz}, 1 \mathrm{H}), 1.56(\mathrm{~d}, J=7.2 \mathrm{~Hz}, 3 \mathrm{H})$. ${ }^{13} \mathrm{C}\left\{{ }^{1} \mathrm{H}\right\}$ NMR $\left(100 \mathrm{MHz}, \mathrm{CDCl}_{3}\right) \delta 162.85,160.41,139.00,138.96,131.57,128.40$, $128.32,128.19,127.81,123.53,115.35,115.14,92.27,82.60,31.76,24.54 ;{ }^{19} \mathrm{~F}$ NMR $\left(376 \mathrm{MHz}, \mathrm{CDCl}_{3}\right) \delta{ }^{19} \mathrm{~F}$ NMR $-117.5(\mathrm{~m})$. HRMS (CI) $\mathrm{m} / z$ calcd for $\mathrm{C}_{16} \mathrm{H}_{12} \mathrm{ClF}(\mathrm{M}+)$ 258.0612 , found 258.0615 .

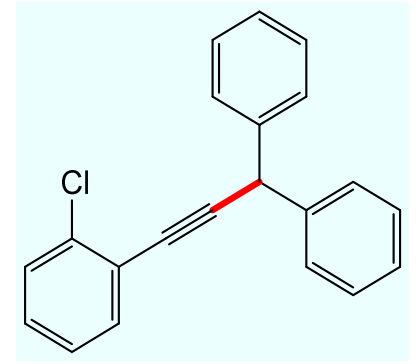

Table 1 entry 12d: (3-(2-chlorophenyl)prop-2-yne-1,1diyl)dibenzene See representative reaction procedure. To a solution of 2-chlorophenylacetylene (11) $(61 \mu \mathrm{L}, 0.5 \mathrm{mmol})$ and $\left[\mathrm{Cl}_{2} \mathrm{NN}\right] \mathrm{Cu}\left(\eta^{2}\right.$-benzene $) \quad(25 \mu \mathrm{L}, 1 \mathrm{~mol} \%)$ in chlorobenzene $(0.5 \mathrm{~mL})$ was added diphenylmethane $(1 \mathrm{~mL})$ 
and ${ }^{t} \mathrm{BuOO}{ }^{t} \mathrm{Bu}(183 \mu \mathrm{L}, 1 \mathrm{mmol})$. $\mathrm{SiO}_{2}$ preparative thin layer chromatography afforded the title compound in $44 \%$ (66 mg) yield as a faint yellow solid. ${ }^{1} \mathrm{H}$ NMR (400 MHz, $\left.\mathrm{CDCl}_{3}\right) \delta 7.80(\mathrm{dt}, J=7.1,1.4 \mathrm{~Hz}, 1 \mathrm{H}), 7.48(\mathrm{dd}, J=7.5,1.9 \mathrm{~Hz}, 6 \mathrm{H}), 7.39$ (dd, $J=7.8$, $1.6 \mathrm{~Hz}, 1 \mathrm{H}), 7.32(\mathrm{dd}, J=8.4,6.8 \mathrm{~Hz}, 5 \mathrm{H}), 7.19(\mathrm{~m}, 4 \mathrm{H}), 5.25(\mathrm{~s}, 1 \mathrm{H}) .{ }^{13} \mathrm{C}\left\{{ }^{1} \mathrm{H}\right\} \mathrm{NMR}$ $\left(100 \mathrm{MHz}, \mathrm{CDCl}_{3}\right) \delta 141.42,136.09,133.31,132.36,130.01,129.15,128.93,128.59$, $128.23,127.91,126.90,126.29,123.35,95.56,81.81,43.98$. (5 carbon atoms missing or coincident). HRMS (CI) $\mathrm{m} / z$ calcd for $\mathrm{C}_{21} \mathrm{H}_{15} \mathrm{Cl}\left(\mathrm{M}^{+}\right)$302.0862, found 302.0865 .

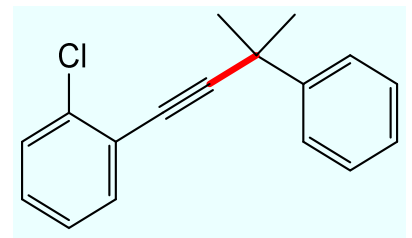

Table 1 entry 12e: 1-chloro-2-(3-methyl-3-phenylbut-1yn-1-yl)benzene. See representative reaction procedure. To a solution of 2-chlorophenylacetylene (11) (61 $\mu \mathrm{L}, 0.5$ mmol) and $\left[\mathrm{Cl}_{2} \mathrm{NN}\right] \mathrm{Cu}\left(\eta^{2}\right.$-benzene $)(25 \mu \mathrm{L}, 1 \mathrm{~mol} \%)$ in chlorobenzene $(0.5 \mathrm{~mL})$ was added cumene $(1 \mathrm{~mL})$ and ${ }^{t} \mathrm{BuOO}{ }^{t} \mathrm{Bu}(183 \mu \mathrm{L}, 1 \mathrm{mmol})$. $\mathrm{SiO}_{2}$ preparative thin layer chromatography afforded the title compound in $52 \%(66 \mathrm{mg})$ yield as a colorless solid. ${ }^{1} \mathrm{H}$ NMR (400 MHz, $\left.\mathrm{CDCl}_{3}\right) \delta 7.62(\mathrm{~m}, 2 \mathrm{H}), 7.45(\mathrm{~m}, 2 \mathrm{H}), 7.34$ $(\mathrm{m}, 2 \mathrm{H}), 7.29(\mathrm{~d}, J=2.2 \mathrm{~Hz}, 1 \mathrm{H}), 7.24(\mathrm{~m}, 1 \mathrm{H}), 1.68(\mathrm{~s}, 6 \mathrm{H}) .{ }^{13} \mathrm{C}\left\{{ }^{1} \mathrm{H}\right\} \mathrm{NMR}(100 \mathrm{MHz}$, $\left.\mathrm{CDCl}_{3}\right) \delta 146.57,136.00,133.14,129.11,128.63,128.25,126.41,126.24,125.62,123.63$, 102.00, 79.07, 36.76, 31.58. (3 carbon atoms missing or coincident). HRMS (CI) m/z calcd for $\mathrm{C}_{17} \mathrm{H}_{15} \mathrm{Cl}\left(\mathrm{M}^{+}\right)$254.0862, found 254.0864.

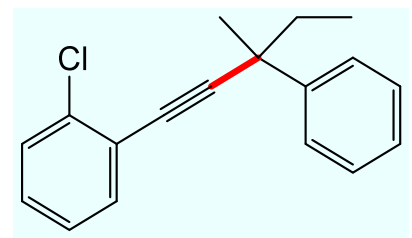

Table 1 entry 12f: 1-chloro-2-(3-methyl-3-phenylpent-1yn-1-yl)benzene. See representative reaction procedure. To a solution of 2-chlorophenylacetylene (11) (61 $\mu \mathrm{L}, 0.5$ $\mathrm{mmol})$ and $\left[\mathrm{Cl}_{2} \mathrm{NN}\right] \mathrm{Cu}\left(\eta^{2}\right.$-benzene $)(25 \mu \mathrm{L}, 1 \mathrm{~mol} \%)$ in chlorobenzene $(0.5 \mathrm{~mL})$ was added isobutylbenzene $(1 \mathrm{~mL})$

and ${ }^{t} \mathrm{BuOO}{ }^{t} \mathrm{Bu}(183 \mu \mathrm{L}, 1 \mathrm{mmol}) . \mathrm{SiO}_{2}$ preparative thin layer chromatography afforded the title compound in $61 \%\left(82 \mathrm{mg}\right.$ ) yield as a faint brown solid. ${ }^{1} \mathrm{H}$ NMR (400 $\mathrm{MHz}$, $\left.\mathrm{CDCl}_{3}\right) \delta 7.61(\mathrm{~m}, 2 \mathrm{H}), 7.50(\mathrm{~m}, 1 \mathrm{H}), 7.36(\mathrm{~m}, 3 \mathrm{H}), 7.20(\mathrm{dt}, J=5.8,3.5 \mathrm{~Hz}, 3 \mathrm{H}), 1.92(\mathrm{q}$, $J=7.4 \mathrm{~Hz}, 2 \mathrm{H}), 1.67(\mathrm{~s}, 3 \mathrm{H}), 0.95(\mathrm{t}, J=7.4 \mathrm{~Hz}, 3 \mathrm{H}) .{ }^{13} \mathrm{C}\left\{{ }^{1} \mathrm{H}\right\}$ NMR $\left(100 \mathrm{MHz}, \mathrm{CDCl}_{3}\right)$ $\delta 145.08,133.25,129.13,128.62,128.14,126.31,126.26,126.22,100.45,80.76,42.00$, 37.11, 29.69, 9.99. (4 carbon atoms missing or coincident). HRMS (CI) $\mathrm{m} / \mathrm{z}$ calcd for $\mathrm{C}_{18} \mathrm{H}_{17} \mathrm{Cl}(\mathrm{M}+)$ 268.1019, found 268.1022. 


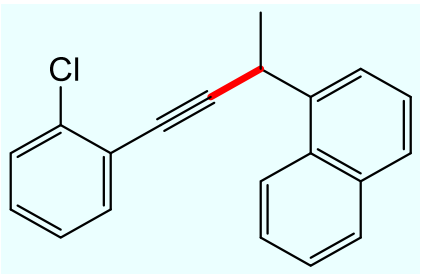

Table 1 entry 12g: 1-(4-(2-chlorophenyl)but-3-yn-2yl)naphthalene. See representative reaction procedure. To a solution of 2-chlorophenylacetylene (11) $(61 \mu \mathrm{L}, 0.5$ $\mathrm{mmol})$ and $\left[\mathrm{Cl}_{2} \mathrm{NN}\right] \mathrm{Cu}\left(\eta^{2}\right.$-benzene) $(25 \mu \mathrm{L}, 1 \mathrm{~mol} \%)$ in chlorobenzene $(0.5 \mathrm{~mL})$ was added 1-ethylnaphthalene $(1$ $\mathrm{mL})$ and ${ }^{t} \mathrm{BuOO}{ }^{t} \mathrm{Bu}(183 \mu \mathrm{L}, 1 \mathrm{mmol}) . \mathrm{SiO}_{2}$ preparative thin layer chromatography afforded the title compound in $69(110 \mathrm{mg}) \%$ yield as a white solid. ${ }^{1} \mathrm{H}$ NMR (400 MHz, $\left.\mathrm{CDCl}_{3}\right) \delta 8.13-7.79(\mathrm{~m}, 5 \mathrm{H}), 7.60(\mathrm{dd}, J=8.5,2.1 \mathrm{~Hz}, 1 \mathrm{H}), 7.45(\mathrm{~m}, 4 \mathrm{H}), 7.20(\mathrm{~m}, 3 \mathrm{H})$, $4.17(\mathrm{q}, J=7.4 \mathrm{~Hz}, 1 \mathrm{H}), 1.69(\mathrm{~d}, J=7.7 \mathrm{~Hz}, 3 \mathrm{H}) .{ }^{13} \mathrm{C}\left\{{ }^{1} \mathrm{H}\right\}$ NMR $\left(100 \mathrm{MHz}, \mathrm{CDCl}_{3}\right) \delta$ $140.27,135.98,133.51,133.24,132.41,129.15,128.75,128.23,127.77,127.57,126.30$, $126.03,125.56,125.56,125.26,123.53,98.02,79.69,32.83,24.27$. HRMS (CI) $m / z$ calcd for $\mathrm{C}_{20} \mathrm{H}_{15} \mathrm{Cl}(\mathrm{M}+)$ 290.0862, found 290.0866 .

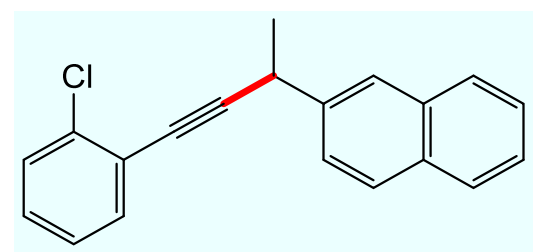

Table 1 entry 12h: 2-(4-(2-chlorophenyl)but-3-yn2-yl)naphthalene. See representative reaction procedure. To a solution of 2-chlorophenylacetylene (11) $(61 \mu \mathrm{L}, 0.5 \mathrm{mmol})$ and $\left[\mathrm{Cl}_{2} \mathrm{NN}\right] \mathrm{Cu}\left(\eta^{2}\right.$-benzene $)$ $(25 \mu \mathrm{L}, 1 \mathrm{~mol} \%)$ in chlorobenzene $(0.5 \mathrm{~mL})$ was added 2-ethylnaphthalene $(1 \mathrm{~mL})$ and ${ }^{t} \mathrm{BuOO}^{t} \mathrm{Bu}(183 \mu \mathrm{L}, 1 \mathrm{mmol}) . \mathrm{SiO}_{2}$ preparative thin layer chromatography afforded the title compound in $76(110 \mathrm{mg}) \%$ yield as an offwhite solid. ${ }^{1} \mathrm{H}$ NMR (400 MHz, $\left.\mathrm{CDCl}_{3}\right) \delta 7.95$ (d, $\left.J=4.0 \mathrm{~Hz}, 1 \mathrm{H}\right), 7.82$ (td, $J=6.1,5.4$, $2.6 \mathrm{~Hz}, 3 \mathrm{H}), 7.59(\mathrm{dd}, J=8.5,1.9 \mathrm{~Hz}, 1 \mathrm{H}), 7.45(\mathrm{~m}, 4 \mathrm{H}), 7.20$ (ddd, $J=7.0,5.0,1.9 \mathrm{~Hz}$, $2 \mathrm{H}), 4.19(\mathrm{q}, J=7.1 \mathrm{~Hz}, 1 \mathrm{H}), 1.68(\mathrm{~d}, J=7.1 \mathrm{~Hz}, 3 \mathrm{H}) .{ }^{13} \mathrm{C}\left\{{ }^{1} \mathrm{H}\right\}$ NMR $\left(100 \mathrm{MHz}, \mathrm{CDCl}_{3}\right)$ $\delta 140.68,135.51,132.42,131.64,130.16,128.26,128.20,127.76,127.59,126.05,125.56$, $125.11,123.70,92.54,82.66,32.61,24.30$. HRMS (CI) $\mathrm{m} / \mathrm{z}$ calcd for $\mathrm{C}_{20} \mathrm{H}_{15} \mathrm{Cl}(\mathrm{M}+)$ 290.0862 , found 290.0864 .

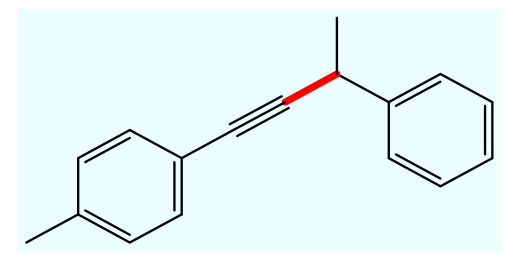

Table 1 entry 13a: 1-methyl-4-(3-phenylbut-1-yn-1yl)benzene. See representative reaction procedure. To a solution of 4-methylphenylacetylene $(63 \mu \mathrm{L}, 0.5$ mmol) and $\left[\mathrm{Cl}_{2} \mathrm{NN}\right] \mathrm{Cu}\left(\eta^{2}\right.$-benzene) $(25 \mu \mathrm{L}, 1 \mathrm{~mol} \%)$ in chlorobenzene $(0.5 \mathrm{~mL})$ was added ethylbenzene $(1 \mathrm{~mL})$ and ${ }^{t} \mathrm{BuOO}{ }^{t} \mathrm{Bu}(183 \mu \mathrm{L}, 1$ 
mmol). $\mathrm{SiO}_{2}$ preparative thin layer chromatography afforded the title compound in $51 \%$ (56 mg) yield as a colorless oil. ${ }^{1} \mathrm{H}$ NMR $\left(400 \mathrm{MHz}, \mathrm{CDCl}_{3}\right) \delta 8.14-7.44(\mathrm{~m}, 5 \mathrm{H}), 7.31-$ $7.26(\mathrm{~m}, 7 \mathrm{H}), 4.17$ (q, $J=7.2 \mathrm{~Hz}, 1 \mathrm{H}), 1.69$ (d, $J=7.4 \mathrm{~Hz}, 3 \mathrm{H}) .{ }^{13} \mathrm{C}\left\{{ }^{1} \mathrm{H}\right\}$ NMR (100 $\left.\mathrm{MHz}, \mathrm{CDCl}_{3}\right) \delta 143.43,137.66,131.44,128.88,128.46,126.88,126.54,120.64,91.75$, $82.44,32.44,24.49,21.35$. (4 carbon atoms missing or coincident). Spectral data matched literature data. ${ }^{13}$

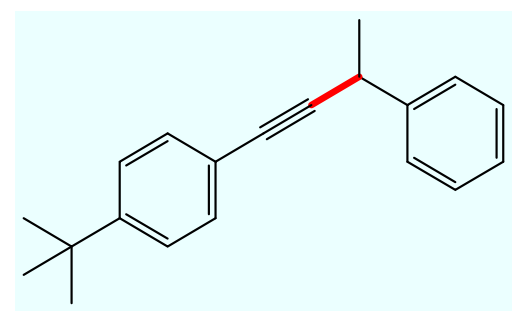

Figure 6 entry 13b: 1-tert-butyl-4-(3-phenylbut-1yn-1-yl)benzene. See representative reaction procedure. To a solution of 4-tert-butylphenylacetylene ( $90 \mu \mathrm{L}, 0.5 \mathrm{mmol})$ and $\left[\mathrm{Cl}_{2} \mathrm{NN}\right] \mathrm{Cu}\left(\eta^{2}\right.$-benzene $) \quad(25 \mu \mathrm{L}, \quad 1 \quad \mathrm{~mol} \%) \quad$ in chlorobenzene $(0.5 \mathrm{~mL})$ was added ethylbenzene $(1 \mathrm{~mL})$ and ${ }^{t} \mathrm{BuOO}{ }^{t} \mathrm{Bu}(183 \mu \mathrm{L}, 1$ $\mathrm{mmol})$. $\mathrm{SiO}_{2}$ preparative thin layer chromatography afforded the title compound in $58 \%$ (76 mg) yield as a colorless oil. ${ }^{1} \mathrm{H}$ NMR $\left(400 \mathrm{MHz}, \mathrm{CDCl}_{3}\right) \delta 8.00(\mathrm{~m}, 1 \mathrm{H}), 7.48(\mathrm{~m}$, $3 \mathrm{H}), 7.37$ (m, 2H), 7.31 (dd, $J=7.9,5.5 \mathrm{~Hz}, 3 \mathrm{H}), 7.24(\mathrm{~m}, 1 \mathrm{H}), 3.97$ (q, $J=7.1 \mathrm{~Hz}, 1 \mathrm{H})$, $1.57(\mathrm{~d}, J=7.1 \mathrm{~Hz}, 3 \mathrm{H}), 1.30(\mathrm{~s}, 9 \mathrm{H}) .{ }^{13} \mathrm{C}\left\{{ }^{1} \mathrm{H}\right\}$ NMR $\left(100 \mathrm{MHz}, \mathrm{CDCl}_{3}\right) \delta 150.89$, 143.46, 131.28, 128.47, 126.90, 126.55, 125.14, 120.69, 91.81, 82.45, 34.66, 32.46, 31.17, 24.57. ( 6 carbon atoms missing or coincident). Spectral data matched literature data. ${ }^{13}$

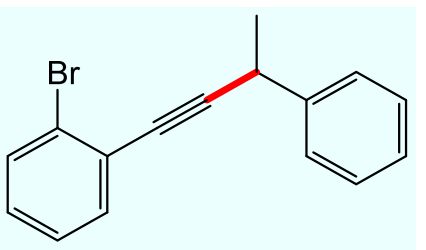

Figure 6 entry 13d: 1-bromo-2-(3-phenylbut-1-yn-1yl)benzene. See representative reaction procedure. To a solution of 2-bromophenylacetylene (90 $\mathrm{mg}, 0.5 \mathrm{mmol}$ ) and $\left[\mathrm{Cl}_{2} \mathrm{NN}\right] \mathrm{Cu}\left(\eta^{2}\right.$-benzene $)(25 \mu \mathrm{L}, 1 \mathrm{~mol} \%)$ in chlorobenzene $(0.5 \mathrm{~mL})$ was added ethylbenzene $(1 \mathrm{~mL})$ and ${ }^{t} \mathrm{BuOO}{ }^{t} \mathrm{Bu}(183 \mu \mathrm{L}, 1$ $\mathrm{mmol}$ ). $\mathrm{SiO}_{2}$ preparative thin layer chromatography afforded the title compound in $61 \%$ $\left(87 \mathrm{mg}\right.$ ) yield as a light yellow solid ${ }^{1} \mathrm{H}$ NMR $\left(400 \mathrm{MHz}, \mathrm{CDCl}_{3}\right) \delta 7.58$ (ddd, $J=11.8$, 7.6, $1.5 \mathrm{~Hz}, 3 \mathrm{H}$ ), 7.48 (ddd, $J=17.3,8.0,1.6 \mathrm{~Hz}, 3 \mathrm{H}), 7.34$ (dd, $J=8.5,6.9 \mathrm{~Hz}, 2 \mathrm{H}$ ), $7.28(\mathrm{~m}, 1 \mathrm{H}), 7.12(\mathrm{td}, J=7.8,1.7 \mathrm{~Hz}, 1 \mathrm{H}), 4.03(\mathrm{q}, J=7.1 \mathrm{~Hz}, 1 \mathrm{H}), 1.62(\mathrm{~d}, J=7.1 \mathrm{~Hz}$, $3 \mathrm{H}) .{ }^{13} \mathrm{C}\left\{{ }^{1} \mathrm{H}\right\}$ NMR $\left(100 \mathrm{MHz}, \mathrm{CDCl}_{3}\right) \delta 142.88,134.50,133.27,132.58,132.28$, $130.35,128.86,128.52,127.01,126.86,126.68,97.50,81.07,77.86,32.72,24.42$. HRMS (CI) $\mathrm{m} / z$ calcd for $\mathrm{C}_{16} \mathrm{H}_{13} \mathrm{Br}(\mathrm{M}+)$ 284.0201, found 284.0203 


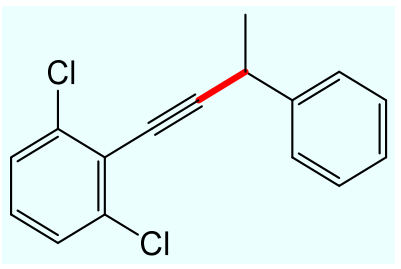

Figure 6 entry 13e: 1,3-dichloro-2-(3-phenylbut-1-yn-1yl)benzene. See representative reaction procedure. To a solution of 2,6-dichlorophenylacetylene ( $86 \mathrm{mg}, 0.5 \mathrm{mmol}$ ) and $\left[\mathrm{Cl}_{2} \mathrm{NN}\right] \mathrm{Cu}\left(\eta^{2}\right.$-benzene $)(25 \mu \mathrm{L}, 1 \mathrm{~mol} \%)$ in chlorobenzene $(0.5 \mathrm{~mL})$ was added ethylbenzene $(1 \mathrm{~mL})$ and ${ }^{t} \mathrm{BuOO}{ }^{t} \mathrm{Bu}(183 \mu \mathrm{L}, 1 \mathrm{mmol}) . \mathrm{SiO}_{2}$ preparative thin layer chromatography afforded the title compound in $51 \%\left(70 \mathrm{mg}\right.$ ) yield as a white solid ${ }^{1} \mathrm{H}$ NMR (400 MHz, $\left.\mathrm{CDCl}_{3}\right) \delta 7.50$ (d, $J=7.6 \mathrm{~Hz}, 2 \mathrm{H}), 7.31(\mathrm{~m}, 5 \mathrm{H}), 7.11(\mathrm{t}, J=8.1 \mathrm{~Hz}, 1 \mathrm{H}), 4.09$ (q, $J=7.1 \mathrm{~Hz}, 1 \mathrm{H}), 1.63$ (d, $J=7.1 \mathrm{~Hz}, 3 \mathrm{H}) .{ }^{13} \mathrm{C}\left\{{ }^{1} \mathrm{H}\right\}$ NMR $\left(100 \mathrm{MHz}, \mathrm{CDCl}_{3}\right) \delta 142.55,137.25,130.04,129.67$, 128.52, 128.46, 128.40, 127.35, 127.00, 126.70, 123.56, 103.84, 32.94, 24.46. (2 carbon atoms missing or coincident). HRMS (CI) $m / z$ calcd for $\mathrm{C}_{16} \mathrm{H}_{12} \mathrm{Cl}_{2}(\mathrm{M}+)$ 274.0316, found 274.0319 .

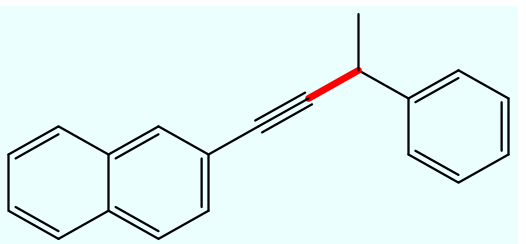

Figure 6 entry 13f: 2-(3-phenylbut-1-yn-1yl)naphthalene. See representative reaction procedure. To a solution of 2-naphtylacetylene (76 $\mathrm{mg}, 0.5 \mathrm{mmol})$ and $\left[\mathrm{Cl}_{2} \mathrm{NN}\right] \mathrm{Cu}\left(\eta^{2}\right.$-benzene $)(25 \mu \mathrm{L}$, $1 \mathrm{~mol} \%)$ in chlorobenzene $(0.5 \mathrm{~mL})$ was added ethylbenzene $(1 \mathrm{~mL})$ and ${ }^{t} \mathrm{BuOO}{ }^{t} \mathrm{Bu}$ (183 $\mu \mathrm{L}, 1 \mathrm{mmol}$ ). $\mathrm{SiO}_{2}$ preparative thin layer chromatography afforded the title compound in $66 \%(84.5 \mathrm{mg})$ yield as a colorless oil. ${ }^{1} \mathrm{H}$ NMR $\left(400 \mathrm{MHz}, \mathrm{CDCl}_{3}\right) \delta 8.02$ (d, $J=53.2 \mathrm{~Hz}, 1 \mathrm{H}), 7.85-7.71(\mathrm{~m}, 4 \mathrm{H}), 7.50(\mathrm{~m}, 5 \mathrm{H}), 7.36(\mathrm{t}, J=7.6 \mathrm{~Hz}, 2 \mathrm{H}), 7.27$ (d, $J=7.3 \mathrm{~Hz}, 1 \mathrm{H}), 4.04(\mathrm{q}, J=7.1 \mathrm{~Hz}, 1 \mathrm{H}), 1.62(\mathrm{~d}, J=7.2 \mathrm{~Hz}, 3 \mathrm{H}) ;{ }^{13} \mathrm{C}\left\{{ }^{1} \mathrm{H}\right\} \operatorname{NMR}(100$ $\left.\mathrm{MHz}, \mathrm{CDCl}_{3}\right) \delta 143.29,133.03,131.17,128.67,128.56,128.47,128.16,127.78,127.68$, $127.59,126.93,126.76,126.67,126.36,126.31,121.04,92.98,82.75,32.57,24.49$. HRMS (CI) $m / z$ calcd for $\mathrm{C}_{20} \mathrm{H}_{16}\left(\mathrm{M}^{+}\right) 256.1252$, found 256.1255 .

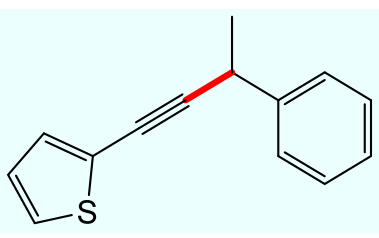

Figure 6 entry 13g: 2-(3-phenylbut-1-yn-1-yl)thiophene. See representative reaction procedure. To a solution of 2-ethynylthiophene $\quad(47 \quad \mu \mathrm{L}, \quad 0.5 \quad \mathrm{mmol}) \quad$ and $\left[\mathrm{Cl}_{2} \mathrm{NN}\right] \mathrm{Cu}\left(\eta^{2}\right.$-benzene) $(25 \mu \mathrm{L}, 1 \mathrm{~mol} \%)$ in chlorobenzene $(0.5 \mathrm{~mL})$ was added ethylbenzene $(1 \mathrm{~mL})$ and ${ }^{t} \mathrm{BuOO}^{t} \mathrm{Bu}(183 \mu \mathrm{L}, 1 \mathrm{mmol}) . \mathrm{SiO}_{2}$ preparative thin layer chromatography afforded the title compound in 53\% (56 mg) yield 
as a yellow oil. ${ }^{1} \mathrm{H}$ NMR $\left(400 \mathrm{MHz}, \mathrm{CDCl}_{3}\right) \delta{ }^{1} \mathrm{H} \mathrm{NMR}\left(399 \mathrm{MHz}, \mathrm{CDCl}_{3}\right) \delta 7.41(\mathrm{~d}, J=$ $7.6 \mathrm{~Hz}, 1 \mathrm{H}), 7.30(\mathrm{~m}, 3 \mathrm{H}), 7.18(\mathrm{~m}, 2 \mathrm{H}), 6.97(\mathrm{~m}, 1 \mathrm{H}), 3.98(\mathrm{q}, J=7.1 \mathrm{~Hz}, 1 \mathrm{H}), 1.56(\mathrm{~d}$, $J=7.1 \mathrm{~Hz}, 3 \mathrm{H}) .{ }^{13} \mathrm{C}\left\{{ }^{1} \mathrm{H}\right\} \mathrm{NMR}\left(100 \mathrm{MHz}, \mathrm{CDCl}_{3}\right) \delta 142.89,131.25,128.56,127.78$, $127.73,126.88,126.75,126.71,126.18,125.64,96.45,75.49,32.70,24.20$. Spectral data matched literature data. ${ }^{14}$

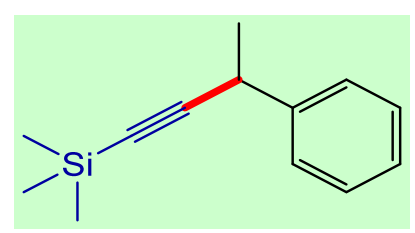

Figure 6 entry 13h: 1-tert-butyl-4-(3-phenylbut-1-yn-1yl)benzene. See representative reaction procedure. To a solution of trimethylsilylacetylene $(69 \mu \mathrm{L}, 0.5 \mathrm{mmol})$ and $\left[\mathrm{Cl}_{2} \mathrm{NN}\right] \mathrm{Cu}\left(\eta^{2}\right.$-benzene) $(25 \mu \mathrm{L}, 1 \mathrm{~mol} \%)$ in chlorobenzene $(0.5 \mathrm{~mL})$ was added ethylbenzene $(1 \mathrm{~mL})$ and ${ }^{t} \mathrm{BuOO}^{t} \mathrm{Bu}(183 \mu \mathrm{L}, 1 \mathrm{mmol}) . \mathrm{SiO}_{2}$ preparative thin layer chromatography afforded the title compound in $67 \%(68 \mathrm{mg})$ yield as a light yellow oil ${ }^{1} \mathrm{H}$ NMR (400 MHz, $\left.\mathrm{CDCl}_{3}\right) \delta 7.35$ (m, 4H), 7.24 (dd, $J=12.2,5.5$ $\mathrm{Hz}, 1 \mathrm{H}), 3.79$ (q, $J=7.2 \mathrm{~Hz}, 1 \mathrm{H}), 1.49$ (d, $J=7.1 \mathrm{~Hz}, 3 \mathrm{H}), 0.19$ (s, 9H). ${ }^{13} \mathrm{C}\left\{{ }^{1} \mathrm{H}\right\} \mathrm{NMR}$ $\left(100 \mathrm{MHz}, \mathrm{CDCl}_{3}\right) \delta 142.88,128.30,128.10,127.44,126.70,126.39,125.87,109.32$, $86.05,32.64,24.46,0.00$. (1 carbon atom missing or coincident). Spectral data matched literature data. ${ }^{14}$

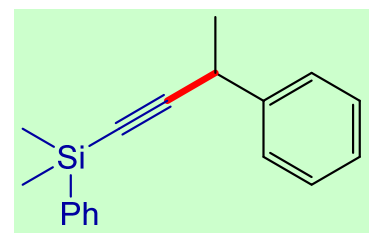

Figure 6 entry 13i: 1-tert-butyl-4-(3-phenylbut-1-yn-1yl)benzene. See representative reaction procedure. To a solution of ethynyldimethyl(phenyl)silane $(80 \mathrm{mg}, 0.5 \mathrm{mmol})$ and $\left[\mathrm{Cl}_{2} \mathrm{NN}\right] \mathrm{Cu}\left(\eta^{2}\right.$-benzene $) \quad(25 \mu \mathrm{L}, \quad 1 \quad \mathrm{~mol} \%)$ in chlorobenzene $(0.5 \mathrm{~mL})$ was added ethylbenzene $(1 \mathrm{~mL})$ and ${ }^{t} \mathrm{BuOO}{ }^{t} \mathrm{Bu}(183 \mu \mathrm{L}, 1$ mmol). $\mathrm{SiO}_{2}$ preparative thin layer chromatography afforded the title compound in $57 \%$ (75mg) yield as a colorless oil. ${ }^{1} \mathrm{H}$ NMR $\left(400 \mathrm{MHz}, \mathrm{CDCl}_{3}\right) \delta 7.32(\mathrm{~m}, 2 \mathrm{H}), 7.05(\mathrm{~m}, 7 \mathrm{H})$, $6.90(\mathrm{~m}, 1 \mathrm{H}), 3.52(\mathrm{q}, J=7.2 \mathrm{~Hz}, 1 \mathrm{H}), 1.20(\mathrm{~d}, J=0.9 \mathrm{~Hz}, 3 \mathrm{H}), 0.09(\mathrm{~s}, 6 \mathrm{H}) .{ }^{13} \mathrm{C}\left\{{ }^{1} \mathrm{H}\right\}$ NMR $\left(100 \mathrm{MHz}, \mathrm{CDCl}_{3}\right) \delta 143.44,138.17,134.28,129.83,129.09,128.38,127.49$, $127.21,112.01,84.82,33.52,25.14,0.00,-0.02$. (4 carbon atoms missing or coincident). Spectral data matched literature data. ${ }^{15}$

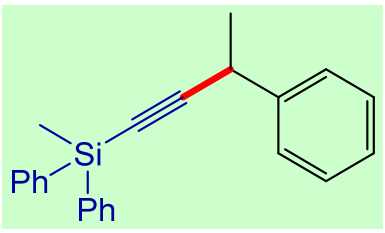

Figure 6 entry 13j: 1-tert-butyl-4-(3-phenylbut-1-yn-1yl)benzene. See representative reaction procedure. To a 
solution of ethynyl(methyl)diphenylsilane (111 $\mathrm{mg}, \quad 0.5 \mathrm{mmol})$ and $\left[\mathrm{Cl}_{2} \mathrm{NN}\right] \mathrm{Cu}\left(\eta^{2}\right.$-benzene $)(25 \mu \mathrm{L}, 1 \mathrm{~mol} \%)$ in chlorobenzene $(0.5 \mathrm{~mL})$ was added ethylbenzene $(1 \mathrm{~mL})$ and ${ }^{t} \mathrm{BuOO}{ }^{t} \mathrm{Bu}(183 \mu \mathrm{L}, 1 \mathrm{mmol}) . \mathrm{SiO}_{2}$ preparative thin layer chromatography afforded the title compound in $73 \%(97 \mathrm{mg})$ yield as a colorless oil ${ }^{1} \mathrm{H}$ NMR (400 MHz, $\left.\mathrm{CDCl}_{3}\right) \delta 7.65(\mathrm{~m}, 4 \mathrm{H}), 7.53(\mathrm{~d}, J=7.0 \mathrm{~Hz}, 1 \mathrm{H}), 7.43$ (d, $J=7.6 \mathrm{~Hz}$, 2H), $7.35(\mathrm{~m}, 4 \mathrm{H}), 7.25(\mathrm{~m}, 2 \mathrm{H}), 3.91$ (q, $J=7.1 \mathrm{~Hz}, 1 \mathrm{H}), 1.57$ (d, $J=7.2 \mathrm{~Hz}, 3 \mathrm{H}), 0.70$ (s, 3H) ${ }^{13} \mathrm{C}\left\{{ }^{1} \mathrm{H}\right\}$ NMR $\left(100 \mathrm{MHz}, \mathrm{CDCl}_{3}\right) \delta 144.49,139.38,137.60,136.37,136.28$, $135.79,131.83,131.37,131.33,130.35,129.87,129.70,129.68,129.53,128.74,128.50$, $114.91,84.41,34.87,26.37,0.00$. (2 carbon atoms missing or coincident). HRMS (CI) $\mathrm{m} / \mathrm{z}$ calcd for $\mathrm{C}_{23} \mathrm{H}_{22} \mathrm{Si}(\mathrm{M}+)$ 326.1491, found 326.1487.

\section{a) Synthesis of silyl acetylenes}

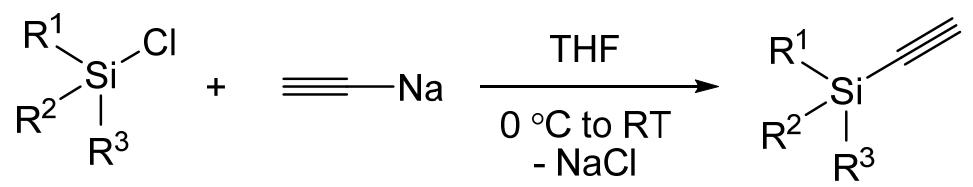

Scheme S14. General synthetic pathway for preparation of terminal silylacetylenes.

According to a modified literature procedure, ${ }^{16}$ the chlorosilane $(6.85 \mathrm{mmol})$ in THF $(10 \mathrm{~mL})$ was added dropwise to a slurry of sodium acetylide (18 $\mathrm{wt} \%$ in xylene/mineral oil, $17.7 \mathrm{~g}, 6.63 \mathrm{mmol})$ in THF $(20 \mathrm{~mL})$ at $0{ }^{\circ} \mathrm{C}$. After stirring overnight at RT, the orange-red suspension was quenched with water and the aqueous layer was extracted with diethyl ether $(5 \times 30 \mathrm{~mL})$. The combined organic phases were washed with a saturated aqueous solution of $\mathrm{NaCl}(100 \mathrm{~mL})$ and dried over $\mathrm{MgSO}_{4}$. The solvent was evaporated, and column chromatographed (5\% ethyl acetate in hexanes) to afford the pure product.

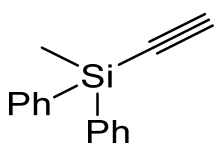

Ethynyl(methyl)diphenylsilane: This alkyne was synthesized according to the general procedure (Scheme S14) to afford the corresponding silylalkyne. ${ }^{1} \mathrm{H}$ NMR $\left(399 \mathrm{MHz}, \mathrm{CDCl}_{3}\right) \delta 7.65$ (dt, $J=$ 
6.3, $1.8 \mathrm{~Hz}, 4 \mathrm{H}), 7.40(\mathrm{~m}, 5 \mathrm{H}), 2.65(\mathrm{~s}, 1 \mathrm{H}), 0.72(\mathrm{~d}, J=1.0 \mathrm{~Hz}, 3 \mathrm{H}) .{ }^{13} \mathrm{C}\left\{{ }^{1} \mathrm{H}\right\}$ NMR $(100$ $\left.\mathrm{MHz}, \mathrm{CDCl}_{3}\right) \delta 136.72,136.69,136.67,132.08,130.26,130.24,130.21,98.65,88.76$, 0.00. (5 carbon atoms missing or coincident). Spectral data matched literature data. ${ }^{17}$

Ethynyldimethyl(phenyl)silane: This alkyne was synthesized according to the general procedure (Scheme S14) to afford the corresponding silylalkyne. ${ }^{1} \mathrm{H}$ NMR $\left(399 \mathrm{MHz}, \mathrm{CDCl}_{3}\right) \delta 7.20(\mathrm{~m}, 2 \mathrm{H}), 7.04(\mathrm{~m}, 3 \mathrm{H})$, $1.20(\mathrm{~d}, J=0.5 \mathrm{~Hz}, 1 \mathrm{H}), 0.00(\mathrm{~s}, 6 \mathrm{H}) .{ }^{13} \mathrm{C}\left\{{ }^{1} \mathrm{H}\right\}$ NMR $\left(100 \mathrm{MHz}, \mathrm{CDCl}_{3}\right) \delta 134.73$, $134.10,130.71,130.34,129.06,128.80,95.89,89.28,1.97,0.00$. (4 carbon atoms missing or coincident). Spectral data matched literature data. ${ }^{18}$ 


\section{Crystallographic details and additional structures.}

Single crystals of each compound $\left[{ }^{i} \mathrm{Pr}_{2} \mathrm{NN}\right] \mathrm{Cu}-\mathrm{O}^{t} \mathrm{Bu}$ (2) (CCDC 1938189), $\left[{ }^{i} \mathrm{Pr}_{2} \mathrm{NN}\right] \mathrm{Cu}-\mathrm{C} \equiv \mathrm{CAr}^{\mathrm{Cl} 2}$ (3) (CCDC 1938190), and $\mathrm{Ph}_{3} \mathrm{C}-\mathrm{C} \equiv \mathrm{CAr}^{\mathrm{Cl} 2}$ (13) (CCDC 1938191) were mounted under pre-cooled Parabar 10312 (previously known as Paratone) on glass fibers and immediately placed in a cold nitrogen stream at 100(2) K prior to data collection. Data for compounds $\mathbf{2}$ and $\mathbf{3}$ were collected on a Bruker D8 Quest equipped with a Photon100 CMOS detector and a MoImS source. Data for compound 13 was collected on a Bruker DUO equipped with an APEXII CCD detector and Mo fine focus sealed tube source. Either full spheres (triclinic) or hemispheres (monoclinic or higher) of data were collected $\left(0.3^{\circ}\right.$ or $0.5^{\circ} \omega^{- \text {scans; }} 2 \theta \max =56^{\circ}$; monochromatic Mo K $\alpha$ radiation, $\lambda=0.7107 \AA$ ) depending on the crystal system and integrated with the Bruker SAINT program. Structure solutions were performed using the SHELXTL/PC suite ${ }^{19}$ and XSEED. ${ }^{20}$ Intensities were corrected for Lorentz and polarization effects and an empirical absorption correction was applied using Blessing's method as incorporated into the program SADABS. ${ }^{21}$ Non-hydrogen atoms were refined with aniostropic thermal parameters and hydrogen atoms were included in idealized positions. Structures were rendered with POV-Ray in XSEED using 50\% probability ellipsoids. Further comments on disorder models:

$\left.{ }^{i} \mathbf{P r}_{2} \mathbf{N N}\right] \mathrm{Cu}-\mathbf{O}^{t} \mathrm{Bu}(\mathbf{2})$.

The datum crystal exhibited psuedo-merohedral twinning. The twin law by rows is $\left(\begin{array}{lll}-1 & 0 & 0\end{array}\right)\left(\begin{array}{lll}0 & -1 & 0\end{array}\right)\left(\begin{array}{lll}0 & 0 & 1\end{array}\right)$ and the BASF refines to approximately 0.50 .

The $\mathrm{OtBu}$ group is disordered over two orientations. The like $\mathrm{Cu}-\mathrm{O}, \mathrm{O}-\mathrm{C}$, and $\mathrm{C}-\mathrm{C}$ distances were restrained to be similar. The methyl carbon atoms were restrained to behave relatively isotropic. One isopropyl group is disordered over two orientations. The like $\mathrm{C}-\mathrm{C}$ distances were restrained to be similar. One 2,6-diisopropyl phenyl group is disordered over two positions. The like $\mathrm{N}-\mathrm{C}$ and $\mathrm{C}-\mathrm{C}$ distances were restrained to be similar. The methyl carbon atoms of one of the isopropyl groups were restrained to behave relatively isotropic. Similar displacement amplitudes were imposed on disordered sites overlapping by less than the sum of van der Waals radii. 


\section{$\left[{ }^{i} \mathrm{Pr}_{2} \mathrm{NN}\right] \mathrm{Cu}-\mathrm{C} \equiv \mathrm{CAr}^{\mathrm{Cl} 2}$ (3).}

Two crystallographically independent molecules of 3 and one pentane solvent molecule are present in the asymmetric unit. On molecule one, the 2,6-dichlorophenyl group is disordered over two positions. The like $\mathrm{C}-\mathrm{C}$ and $\mathrm{C}-\mathrm{Cl}$ distances were restrained to be similar. On molecule two, three of the isopropyl groups are disordered over two positions. The like C-C distances were restrained to be similar. Similar displacement amplitudes were imposed on disordered sites overlapping by less than the sum of van der Waals radii. 


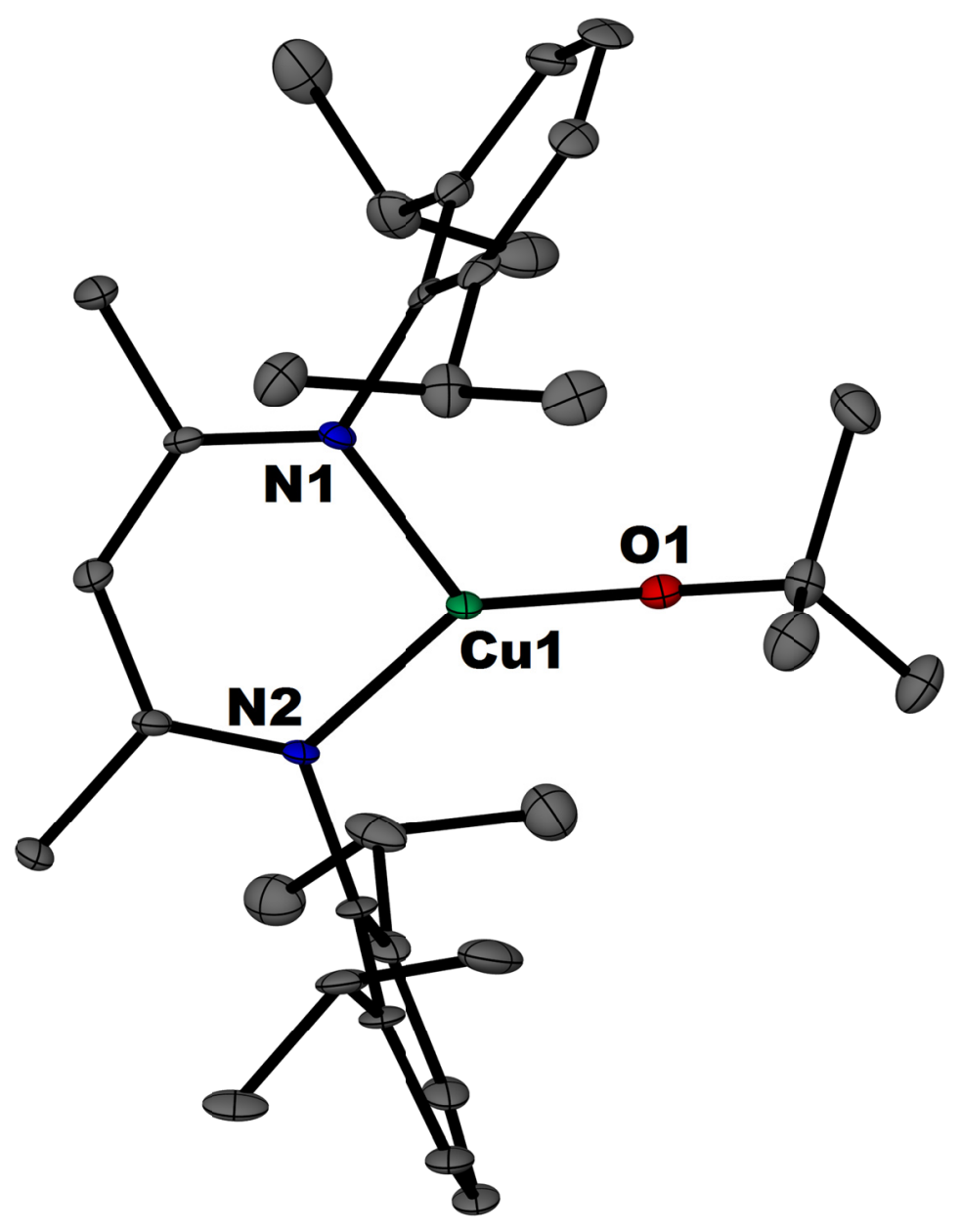

Figure S20. Molecular structure of $\left[{ }^{i} \mathrm{Pr}_{2} \mathrm{NN}\right] \mathrm{Cu}-\mathrm{O}^{t} \mathrm{Bu}$ (2) (CCDC 1938189). The thermal ellipsoid plots are drawn at $30 \%$ probability level. $\mathrm{N} 1$ ' is a symmetry generated atom. Hydrogen atoms are omitted for clarity. Only the major component of disordered sites is shown. Selected bond distances $(\AA)$ and angles $\left({ }^{\circ}\right)$ : Cu1-N1 1.877(6), Cu1-N2 1.894(6), Cu1-O1 1.809(5), Cu1-O1' 1.806(8), N1-Cu1-N2 95.98(16), N1-Cu1-O1 125.3(3), $\mathrm{N} 1-\mathrm{Cu} 1-\mathrm{O}^{\prime}$ 129.8(4), N2-Cu1-O1 134.8(3), N2-Cu1-O1' 124.9(5). 


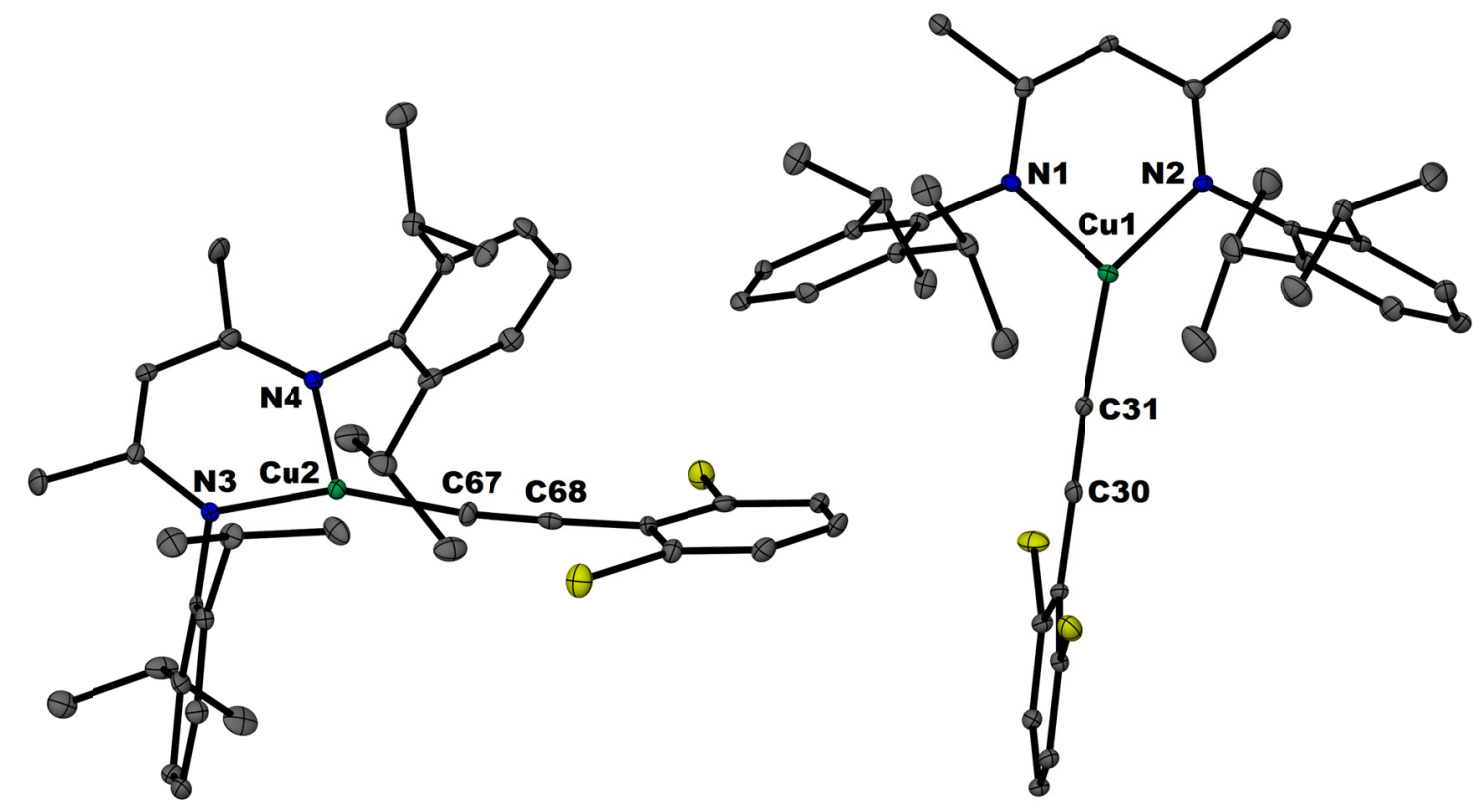

Figure S21. Molecular structure of $\left[{ }^{i} \mathrm{Pr}_{2} \mathrm{NN}\right] \mathrm{Cu}-\mathrm{C} \equiv \mathrm{CAr}{ }^{\mathrm{Cl} 2}\left(\mathrm{Ar}=2,6-\mathrm{Cl}_{2}-\mathrm{C}_{6} \mathrm{H}_{3}\right)$ (3) (CCDC 1938190). The thermal ellipsoid plots are drawn at $30 \%$ probability level. Hydrogen atoms are omitted for clarity. Selected bond distances $(\AA)$ and angles $\left(^{\circ}\right)$; Molecule 1: Cu1-N1 1.907(4), Cu1-N2 1.859(4), Cu1-C30 1.887(5), C31-C30-Cu1 174.3(5), N2-Cu1-C30 142.5(2), N1-Cu1-C30 122.0(2). Molecule 2: Cu2-N3 1.861(4), $\mathrm{Cu} 2-\mathrm{N} 3$ 1.861(4), Cu2-N4 1.903(4), Cu2-C67 1.872(6), C68-C67-Cu2 170.1(6), N3-Cu2-C67 150.6(2), N4-Cu2-C67 112.9(2). 


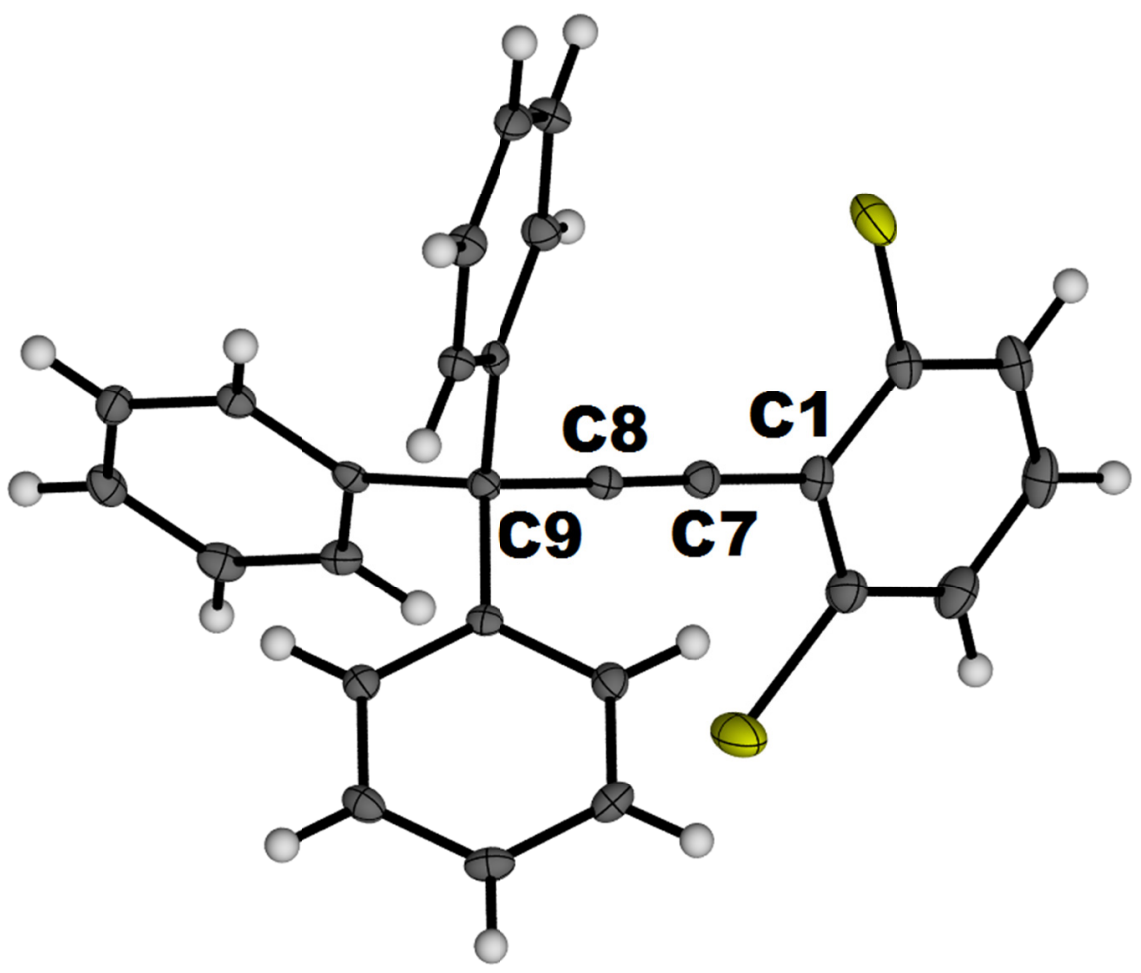

Figure S22. Solid state structure of $\mathrm{Ph}_{3} \mathrm{C}-\mathrm{C} \equiv \mathrm{CAr}{ }^{\mathrm{Cl} 2}$ (13) (CCDC 1938191). Thermal ellipsoids are shown at $30 \%$ probability. Selected bond distances $(\AA)$ and angles $\left(^{\circ}\right)$ : C1-C7 1.432(2), C7-C8 1.197(2), C8-C9 1.482(2), C8-C7-C1 178.79(16), C7-C8-C9 179.04(16). 


\section{References for Synthetic and Crystallographic Details}

(1) F. Neese, W. G. Zumft, W. E. Antholine, P. M. H. Kroneck, J. Am. Chem. Soc. 1996, 118, 8692-8699.

(2) D. L. Musso, M. J. Clarke, J. L. Kelley, G. E. Boswelld, G, Chen, Org. Biomol. Chem. 2003, 1, 498-506.

(3) Y. M. Badiei, T. H. Warren, K. P. Chiang, P. L. Holland, Inorg. Synth. 2010, 35, 50-53.

(4) D. Adhikari, B. L. Tran, F. J. Zuno-Cruz, G. Sanchez Cabrera, D. J. Mindiola, K. P. Chiang, R. E. Cowley, T. R. Dugan, P. L. Holland, Inorg. Synth. 2010, 35, 8-13.

(5) D. J. E. Spencer, N. W. Aboelella, A. M. Reynolds, P. L. Holland, W. B. Tolman, J. Am. Chem. Soc. 2002, 124, 2108-2109.

(6) T. K. Salvador, C. H. Arnett, S. Kundu, N. G. Sapiezynski, J. A. Bertke, M. R. Boroujeni, T. H. Warren, J. Am. Chem. Soc. 2016, 138, 16580-16583.

(7) S. Kundu, W. Y. Kim, J. A. Bertke, T. H. Warren, J. Am. Chem. Soc. 2017, $139,1045-1048$.

(8) A. Bakhoda, Q. Jiang, Y. M. Badiei, J. A. Bertke, T. R. Cundari, T. H. Warren, , Angew. Chem. Int. Ed. 2019, 58, 3421-3425.

(9) S. Wise, Y. M. Badieie, R. T. Gephart, S. Mossin, M. S. Varonka, M. M. Melzer, K. Meyer, T. R. Cundari, T. H. Warren, Angew. Chem. Int. Ed. 2010, 49, 8850-8855.

(10) M. M. Melzer, S. Mossin, A. J. P. Cardenas, K. D. Williams, S. Zhang, K. Meyer, T. H. Warren, Inorg. Chem. 2012, 51, 8658-8660.

(11) M. Gomberg, J. Am. Chem. Soc. 1900, 22, 757-771.

(12) A. Kumar Morri, Y. Thummala, V. Ramana Doddi, Org. Lett. 2015, 17, 4640-4643.

(13) H. Fang, Z. Yang, L. Zhang, W. Wang, Y. Li, X. Xu, S. Zhou, Org. Lett. 2016, 18, 6022-6025.

(14) M. Guisán-Ceinos, V. Martín-Heras, M. Tortosa, J. Am. Chem. Soc. 2017, $139,8448-8451$. 
(15) L. Antonella Aronica, C. Mazzoni, A. Maria Caporusso, Tetrahedron, 2010, 66, 265-273.

(16) S. E. Denmark, K. L. Habermas, G. A. Hite, T. K. Jones, Tetrahedron, 1986, 42, 2821-2829.

(17) C. S. Kraihanzel, M. L. Losee, J. Organomet. Chem. 1967, 10, 427-437.

(18) J. H. Lamm, P. Niermeier, A. Mix, J. Chmiel, B. Neumann, H. G. Stammler, N. W. Mitzel, Angew. Chem. Int. Ed. 2014, 53, 7938-7942.

(19) SHELXTL-PC, Vers. 5.10; 1998, Bruker-Analytical X-ray Services, Madison, WI; Sheldrick, G. M. SHELX-97, Universität Göttingen, Göttingen, Germany.

(20) Barbour, L. XSEED, 1999.

(21) SADABS; G. M. Sheldrick, 1996, based on the method described in R. H. Blessing, Acta Crystallogr. Sect. A 1995, 51, 33-38. 


\section{Computational Details}

Gaussian $16^{22}$ was used to optimize the structures, calculate single point geometries, and also find the vibrational frequencies and reaction free energies. Visualization, structural and molecular orbital analyses were done using Chemcraft 1.6, Chimera, and CCDCMercury. ${ }^{23-25}$ Twelve complexes were studied: $\left[{ }^{i} \operatorname{Pr}_{2} \mathrm{NN}\right] \mathrm{Cu}-\mathrm{C} \equiv \mathrm{CAr}^{\mathrm{Cl} 2} \quad$ (3), $\left\{\left[{ }^{i} \mathrm{Pr}_{2} \mathrm{NN}\right] \mathrm{Cu}-\mathrm{C} \equiv \mathrm{CAr}^{\mathrm{Cl} 2}\right\}^{-} \quad$ (4), $\left\{\left[{ }^{i} \mathrm{Pr}_{2} \mathrm{NN}\right] \mathrm{Cu}-\mathrm{C} \equiv \mathrm{CAr}^{\mathrm{Cl} 2}\right\}^{+}$(8), $\left[{ }^{i} \operatorname{Pr}_{2} \mathrm{NN}\right] \mathrm{Cu}\left(\mathrm{C} \equiv \mathrm{CAr}{ }^{\mathrm{Cl} 2}\right)_{2}$, $\left\{\left[{ }^{i} \mathrm{Pr}_{2} \mathrm{NN}\right] \mathrm{Cu}\left(\mathrm{C} \equiv \mathrm{CAr}{ }^{\mathrm{Cl} 2}\right)_{2}\right\}^{-}, \quad\left[{ }^{i} \mathrm{Pr}_{2} \mathrm{NN}\right] \mathrm{Cu}\left({ }^{\mathrm{C} 12} \mathrm{ArC} \equiv \mathrm{C}-\mathrm{C} \equiv \mathrm{CAr}{ }^{\mathrm{Cl} 2}\right)$, $\left[{ }^{i} \mathrm{Pr}_{2} \mathrm{NN}\right] \mathrm{Cu}\left(\mathrm{C} \equiv \mathrm{CAr}^{\mathrm{Cl} 2}\right)(\mathrm{NCMe}), \quad\left\{\left[{ }^{i} \mathrm{Pr}_{2} \mathrm{NN}\right] \mathrm{Cu}\left(\mathrm{C} \equiv \mathrm{CAr}^{\mathrm{Cl} 2}\right)(\mathrm{NCMe})\right\}^{+}$, $\left\{\left[{ }^{i} \mathrm{Pr}_{2} \mathrm{NN}\right] \mathrm{Cu}\left(\mathrm{C} \equiv \mathrm{CAr}{ }^{\mathrm{Cl} 2}\right)(\mathrm{Ph})\right\}^{-}, \quad\left[{ }^{i} \mathrm{Pr}_{2} \mathrm{NN}\right] \mathrm{Cu}\left(\mathrm{C} \equiv \mathrm{CAr}^{\mathrm{Cl} 2}\right)(\mathrm{Ph}), \quad\left[{ }^{i} \mathrm{Pr}_{2} \mathrm{NN}\right] \mathrm{Cu}\left({ }^{\mathrm{Cl} 2} \mathrm{ArC} \equiv \mathrm{C}-\mathrm{Ph}\right)$, $\left[{ }^{i} \mathrm{Pr}_{2} \mathrm{NN}\right] \mathrm{Cu}(\mathbf{1}),\left[{ }^{i} \mathrm{Pr}_{2} \mathrm{NN}\right] \mathrm{Cu}\left(\eta^{2}-\mathrm{C}_{6} \mathrm{H}_{6}\right)\left(\mathbf{1}-\mathbf{C}_{6} \mathbf{H}_{6}\right)$, and $\left[{ }^{i} \operatorname{Pr}_{2} \mathrm{NN}\right] \mathrm{Cu}(\mathrm{NCMe})(\mathbf{1 - N C M e})$. Seven small molecules $\left({ }^{\mathrm{Cl} 2} \mathrm{ArC} \equiv \mathrm{C} \cdot\right.$ radical, ${ }^{\mathrm{Cl} 2} \mathrm{ArC} \equiv \mathrm{C}^{-}$anion, ${ }^{\mathrm{Cl} 2} \mathrm{ArC} \equiv \mathrm{C}-\mathrm{C} \equiv \mathrm{CAr}{ }^{\mathrm{Cl} 2}$ (5), $\mathrm{Ph}^{-}$anion, ${ }^{\mathrm{Cl} 2} \mathrm{ArC} \equiv \mathrm{C}-\mathrm{Ph}(\mathbf{1 0}), \mathrm{C}_{6} \mathrm{H}_{6}$ and $\left.\mathrm{MeCN}\right)$ were also calculated to analyze the thermodynamics of the reactions between the complexes.

The $\mathrm{BP} 86^{26}$ functional in conjunction with the $6-311+\mathrm{G}(\mathrm{d})^{27-34}$ basis set $(5 \mathrm{~d} 7 \mathrm{f}$ spherical harmonics) was used for optimization of all geometries. At the BP86/6-311+G(d) stationary points, single point energies were calculated using BP86 with the 6$311++\mathrm{G}(\mathrm{d}, \mathrm{p})^{27-34}$ basis set, in benzene or acetonitrile using the implicit $\mathrm{SMD}^{35}$ solvent model (Table S7 and S8) and adding dispersion corrections with the keyword, empirical dispersion $=$ GD3BJ. ${ }^{36}$ 
Table S7. Gibbs free energies (BP86/6-311+G(d)/gas//BP86+GD3BJ/6$311++\mathrm{G}(\mathrm{d}, \mathrm{p}) / \mathrm{SMD}$-benzene) at $298.15 \mathrm{~K}$ of the computed molecules. $[\mathrm{Cu}]=\left[{ }^{i} \mathrm{Pr}_{2} \mathrm{NN}\right] \mathrm{Cu}$ fragment.

\begin{tabular}{|c|c|c|c|}
\hline Compound & Charge & Multiplicity & Free Energy \\
\hline$\left[\mathrm{Cu}^{\mathrm{II}}\right]-\mathrm{C} \equiv \mathrm{CAr}^{\mathrm{Cl} 2} \mathrm{Y}$-shaped & 0 & 2 & -4107.176337 \\
\hline$\left[\mathrm{Cu}^{\mathrm{II}}\right]-\mathrm{C} \equiv \mathrm{CAr}^{\mathrm{C} 12} \mathrm{~T}$-shaped & 0 & 2 & -4107.175698 \\
\hline$\left\{\left[\mathrm{Cu}^{\mathrm{I}}\right]-\mathrm{C} \equiv \mathrm{CAr}^{\mathrm{Cl} 2}\right\}^{-}$ & -1 & 1 & -4107.303284 \\
\hline$\left\{\left[\mathrm{Cu}^{\mathrm{III}}\right]-\mathrm{C} \equiv \mathrm{CAr}^{\mathrm{Cl} 2}\right\}^{+}$ & +1 & 1 & -4106.974526 \\
\hline$\left\{\left[\mathrm{Cu}^{\mathrm{III}}\right]-\mathrm{C} \equiv \mathrm{CAr}^{\mathrm{Cl} 2}\right\}^{+}$ & +1 & 3 & -4106.962277 \\
\hline$\left\{\left[\mathrm{Cu}^{\mathrm{II}}\right]\left(\mathrm{C} \equiv \mathrm{CAr}^{\mathrm{Cl} 2}\right)_{2}\right\}^{-}$ & -1 & 2 & -5334.435372 \\
\hline$\left[\mathrm{Cu}^{\mathrm{III}}\right]\left(\mathrm{C} \equiv \mathrm{CAr}^{\mathrm{Cl} 2}\right)_{2}$ constrained & 0 & 1 & -5334.302247 \\
\hline$\left[\mathrm{Cu}^{\mathrm{III}}\right]\left(\mathrm{C} \equiv \mathrm{CAr}^{\mathrm{Cl} 2}\right)_{2}$ & 0 & 3 & -5334.283934 \\
\hline$\left[\mathrm{Cu}^{\mathrm{I}}\right]\left({ }^{\mathrm{C} 12} \mathrm{ArC} \equiv \mathrm{C}-\mathrm{C} \equiv \mathrm{CAr}^{\mathrm{Cl} 2}\right)$ & 0 & 1 & -5334.387384 \\
\hline$\left[\mathrm{Cu}^{\mathrm{II}}\right]\left(\mathrm{C} \equiv \mathrm{CAr}^{\mathrm{Cl}}\right)(\mathrm{NCMe})$ & 0 & 2 & -4239.942250 \\
\hline$\left\{\left[\mathrm{Cu}^{\mathrm{III}}\right]\left(\mathrm{C} \equiv \mathrm{CAr}^{\mathrm{Cl} 2}\right)(\mathrm{NCMe})\right\}^{+}$ & +1 & 1 & -4239.744184 \\
\hline$\left\{\left[\mathrm{Cu}^{\mathrm{III}}\right]\left(\mathrm{C} \equiv \mathrm{CAr}^{\mathrm{Cl} 2}\right)(\mathrm{NCMe})\right\}^{+}$ & +1 & 3 & -4239.729845 \\
\hline$\left\{\left[\mathrm{Cu}^{\mathrm{II}}\right]\left(\mathrm{C} \equiv \mathrm{CAr}^{\mathrm{Cl} 2}\right)(\mathrm{Ph})\right\}^{-}$ & -1 & 2 & -4338.907401 \\
\hline$\left[\mathrm{Cu}^{\mathrm{III}}\right]\left(\mathrm{C} \equiv \mathrm{CAr}^{\mathrm{Cl} 2}\right)(\mathrm{Ph})$ constrained & 0 & 1 & -4338.781498 \\
\hline$\left[\mathrm{Cu}^{\mathrm{I}}\right]\left({ }^{\mathrm{Cl} 2} \mathrm{ArC} \equiv \mathrm{C}-\mathrm{Ph}\right)$ & 0 & 1 & -4338.867383 \\
\hline$\left[\mathrm{Cu}^{\mathrm{I}}\right]$ & 0 & 1 & -2879.997059 \\
\hline$\left[\mathrm{Cu}^{\mathrm{I}}\right]\left(\eta^{2}-\mathrm{C}_{6} \mathrm{H}_{6}\right)$ & 0 & 1 & -3112.269794 \\
\hline${ }^{\mathrm{C} 12} \mathrm{ArC} \equiv \mathrm{C} \cdot$ radical & 0 & 2 & -1227.062868 \\
\hline${ }^{\mathrm{C} 12} \mathrm{ArC} \equiv \mathrm{C}^{-}$ & -1 & 1 & -1227.23388 \\
\hline${ }^{\mathrm{Cl} 2} \mathrm{ArC} \equiv \mathrm{C}-\mathrm{C} \equiv \mathrm{CAr}^{\mathrm{Cl} 2}$ & 0 & 1 & -2454.355774 \\
\hline $\mathrm{Ph}^{-}$ & -1 & 1 & -231.6738068 \\
\hline${ }^{\mathrm{Cl} 2} \mathrm{ArC} \equiv \mathrm{C}-\mathrm{Ph}$ & 0 & 1 & -1458.837783 \\
\hline $\mathrm{C}_{6} \mathrm{H}_{6}$ & 0 & 1 & -232.2537212 \\
\hline
\end{tabular}

Note: Multiple attempts with different initial geometry guesses to optimize $\left[^{i} \mathrm{Pr}_{2} \mathrm{NN}\right] \mathrm{Cu}^{I I I}\left(\mathrm{C} \equiv \mathrm{CAr}^{\mathrm{Cl}}\right)_{2}$ (Figure S32) and $\left[^{i} \mathrm{Pr}_{2} \mathrm{NN}\right] \mathrm{Cu}{ }^{I I I}\left(\mathrm{C} \equiv \mathrm{CAr}^{\mathrm{Cl}}\right)(\mathrm{Ph})$ (Figure S35) at the BP86/6-311+G(d) level of theory were not successful due to very facile reductive elimination affording $\left.\left[{ }^{i} \mathrm{Pr}_{2} \mathrm{NN}\right] \mathrm{Cu} u^{I}{ }^{C l 2} \mathrm{C} \equiv \mathrm{C}-\mathrm{C} \equiv \mathrm{CAr}{ }^{C l 2}\right)$ or $\left[{ }^{i} \mathrm{Pr}_{2} \mathrm{NN}\right] \mathrm{Cu} u^{I}\left({ }^{2 l 2} \mathrm{ArC} \equiv \mathrm{C}-\mathrm{Ph}\right)$ respectively. The only converged isomer of $\left.{ }^{i} \mathrm{Pr}_{2} \mathrm{NN}\right] \mathrm{Cu} u^{I I I}\left(\mathrm{C} \equiv \mathrm{CAr}^{\mathrm{Cl}}\right)_{2}$ at this level of theory is in the triplet state and tetrahedral geometry. A constrained (C30-C38 distances fixed to $2.783 A$ based off the C30-C38 distance of $\left.\left\{\left[{ }^{i} \operatorname{Pr}_{2} \mathrm{NN}\right] \mathrm{Cu}^{\mathrm{II}}\left(\mathrm{C} \equiv \mathrm{CAr}^{\mathrm{Cl} 2}\right)_{2}\right\}^{-}\right)$ $\left[{ }^{i} \mathrm{Pr}_{2} \mathrm{NN}\right] \mathrm{Cu}{ }^{I I I}\left(\mathrm{C} \equiv \mathrm{CAr}^{\mathrm{Cl2}}\right)_{2}$ (charge $=0$, multiplicity = 1) has been employed as an estimate to calculate the thermodynamics of model reactions (Table S5). A constrained (C30-C38 distances fixed to $3.042 \AA$ a based off the C30-C38 distance of $\left.\left\{\left[^{i} \mathrm{Pr}_{2} \mathrm{NN}\right] \mathrm{Cu} u^{I I}\left(\mathrm{C} \equiv \mathrm{CAr}{ }^{\mathrm{Cl}}\right)(\mathrm{Ph})\right\}^{-}\right)\left[^{i} \mathrm{Pr}_{2} \mathrm{NN}\right] \mathrm{Cu}{ }^{I I I}\left(C \equiv C A r^{C l 2}\right)(\mathrm{Ph})$ (charge $=0$, multiplicity $=1)$ has been employed as an estimate to calculate the thermodynamics of model reactions (Table S5). 
Table S8. Gibbs free energies (BP86/6-311+G(d)/gas//BP86+GD3BJ/6$311++\mathrm{G}(\mathrm{d}, \mathrm{p}) / \mathrm{SMD}$-acetonitrile) at $298.15 \mathrm{~K}$ of the computed molecules. $[\mathrm{Cu}]=$ $\left[{ }^{i} \mathrm{Pr}_{2} \mathrm{NN}\right] \mathrm{Cu}$ fragment.

\begin{tabular}{|c|c|c|c|}
\hline Compound & Charge & Multiplicity & Free Energy \\
\hline$\left[\mathrm{Cu}^{\mathrm{II}}\right]-\mathrm{C} \equiv \mathrm{CAr}{ }^{\mathrm{Cl} / 2} \mathrm{Y}$-shaped & 0 & 2 & -4107.184768 \\
\hline$\left[\mathrm{Cu}^{\mathrm{Il}}\right]-\mathrm{C} \equiv \mathrm{CAr}^{\mathrm{C} 12}$ T-shaped & 0 & 2 & -4107.183957 \\
\hline$\left\{\left[\mathrm{Cu}^{1}\right]-\mathrm{C} \equiv \mathrm{CAr}^{\mathrm{Cl} 2}\right\}^{-}$ & -1 & 1 & -4107.334457 \\
\hline$\left\{\left[\mathrm{Cu}^{\mathrm{III}}\right]-\mathrm{C} \equiv \mathrm{CAr}^{\mathrm{C} / 1}\right\}^{+}$ & +1 & 1 & -4107.000484 \\
\hline$\left\{\left[\mathrm{Cu}^{\mathrm{III}}\right]-\mathrm{C} \equiv \mathrm{CAr}^{\mathrm{Cl} 2}\right\}^{+}$ & +1 & 3 & -4106.991636 \\
\hline$\left\{\left[\mathrm{Cu}^{\mathrm{II}}\right]\left(\mathrm{C} \equiv \mathrm{CAr}^{\mathrm{Cl} 1}\right)_{2}\right\}^{-}$ & -1 & 2 & -5334.469404 \\
\hline$\left[\mathrm{Cu}^{\mathrm{III}}\right]\left(\mathrm{C} \equiv \mathrm{CAr}^{\mathrm{Cl} 2}\right)_{2}$ constrained & 0 & 1 & -5334.313466 \\
\hline$\left[\mathrm{Cu}^{\mathrm{III}}\right]\left(\mathrm{C} \equiv \mathrm{CAr}^{\mathrm{Cl} 2}\right)_{2}$ & 0 & 3 & -5334.296998 \\
\hline$\left[\mathrm{Cu}^{\mathrm{l}]}\right]\left({ }^{\mathrm{Cl} 2} \mathrm{ArC} \equiv \mathrm{C}-\mathrm{C} \equiv \mathrm{CAr}^{\mathrm{Cl} 2}\right)$ & 0 & 1 & -5334.392118 \\
\hline$\left[\mathrm{Cu}^{\mathrm{Il}}\right]\left(\mathrm{C} \equiv \mathrm{CAr}^{\mathrm{Cl} 2}\right)(\mathrm{NCMe})$ & 0 & 2 & -4239.951139 \\
\hline$\left\{\left[\mathrm{Cu}^{\mathrm{III}}\right]\left(\mathrm{C} \equiv \mathrm{CAr}^{\mathrm{Cl} 2}\right)(\mathrm{NCMe})\right\}^{+}$ & +1 & 1 & -4239.76856 \\
\hline$\left\{\left[\mathrm{Cu}^{\mathrm{III}}\right]\left(\mathrm{C} \equiv \mathrm{CAr}^{\mathrm{Cl} 2}\right)(\mathrm{NCMe})\right\}^{+}$ & +1 & 3 & -4239.754284 \\
\hline$\left[\mathrm{Cu}^{1}\right]$ & 0 & 1 & -2880.003875 \\
\hline$\left[\mathrm{Cu}^{1}\right](\mathrm{NCMe})$ & 0 & 1 & -3012.809954 \\
\hline${ }^{\mathrm{Cl} 2} \mathrm{ArC} \equiv \mathrm{C}$. & 0 & 2 & -1227.069524 \\
\hline${ }^{\mathrm{C} 12} \mathrm{ArC} \equiv \mathrm{C}^{-}$ & -1 & 1 & -1227.276933 \\
\hline${ }^{\mathrm{Cl} 2} \mathrm{ArC} \equiv \mathrm{C}-\mathrm{C} \equiv \mathrm{CAr}{ }^{\mathrm{Cl} 2}$ & 0 & 1 & -2454.355807 \\
\hline $\mathrm{MeCN}$ & 0 & 1 & -132.7847598 \\
\hline
\end{tabular}

Note: Multiple attempts with different initial geometry guesses to optimize $\left.{ }^{i} \mathrm{Pr}_{2} \mathrm{NN}\right] \mathrm{Cu}{ }^{\mathrm{III}}\left(\mathrm{C} \equiv \mathrm{CAr}^{\mathrm{Cl}}\right)_{2}$ (Figure S32) at the BP86/6-311+G(d) level of theory were not successful due to very facile reductive elimination affording $\left[{ }^{i} \mathrm{Pr}_{2} \mathrm{NN}\right] \mathrm{Cu}^{I}\left({ }^{\mathrm{Cl}} \mathrm{C} \equiv \mathrm{C}-\mathrm{C} \equiv \mathrm{CAr}{ }^{\mathrm{Cl}}\right)$. The only converged isomer of $\left.{ }_{[}^{i} \mathrm{Pr}_{2} \mathrm{NN}\right] \mathrm{Cu}{ }^{I I I}\left(\mathrm{C} \equiv \mathrm{CAr}^{\mathrm{Cl}}\right)_{2}$ at this level of theory is in the triplet state and tetrahedral geometry. A constrained (C30-C38 distances fixed to 2.783 A based off the C30-C38 distance of $\left.\left\{\left[{ }^{i} \mathrm{Pr}_{2} \mathrm{NN}\right] \mathrm{Cu}{ }^{\mathrm{II}}\left(\mathrm{C} \equiv \mathrm{CAr}^{\mathrm{Cl}}\right)_{2}\right\}^{-}\right) \quad\left[{ }^{i} \mathrm{Pr}_{2} \mathrm{NN}\right] C \mathrm{Cu}^{\mathrm{III}}\left(\mathrm{C} \equiv \mathrm{CAr}^{\mathrm{Cl} 2}\right)_{2} \quad$ (charge $=0$, multiplicity $=1)$ has been employed as an estimate to calculate the thermodynamics of model reactions (Table $S 6)$. 


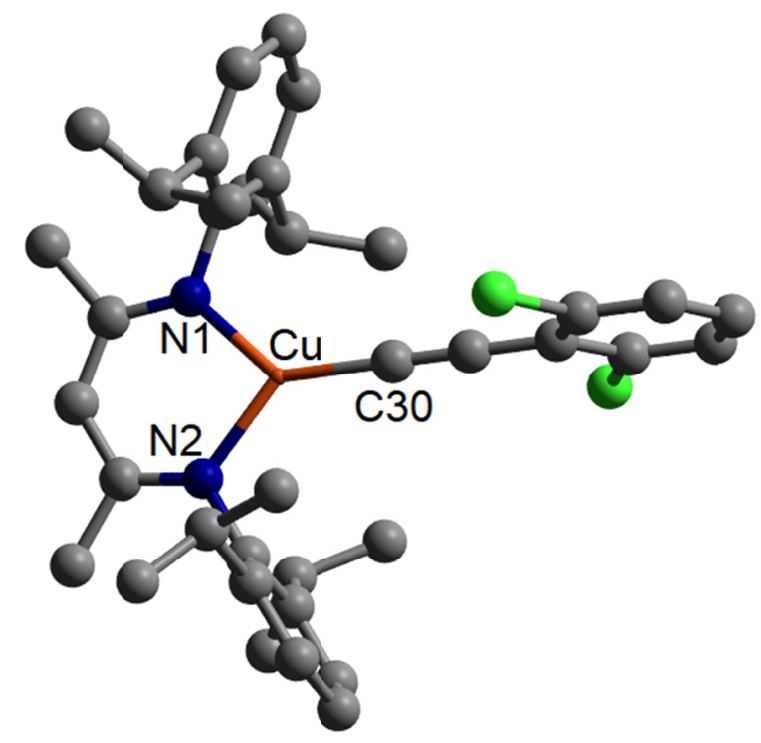

Figure S23. Geometry optimized structure of $\mathrm{Y}$-shaped $\left[{ }^{i} \mathrm{Pr}_{2} \mathrm{NN}\right] \mathrm{Cu}-\mathrm{C} \equiv \mathrm{CAr}{ }^{\mathrm{Cl} 2}$ (3) at $\mathrm{BP} 86 / 6-311+\mathrm{G}(\mathrm{d})$ level of theory with charge $=0$, multiplicity $=2$. Selected calculated bond distances $(\AA)$ and angles $\left(^{\circ}\right)$ for 3: Cu1-N1, 1.935; Cu1-N2, 1.935; Cu1-C30, 1.875; N1-Cu1-N2, 95.08; N1-Cu1-C30, 132.46; N2-Cu1-C30, 132.46.

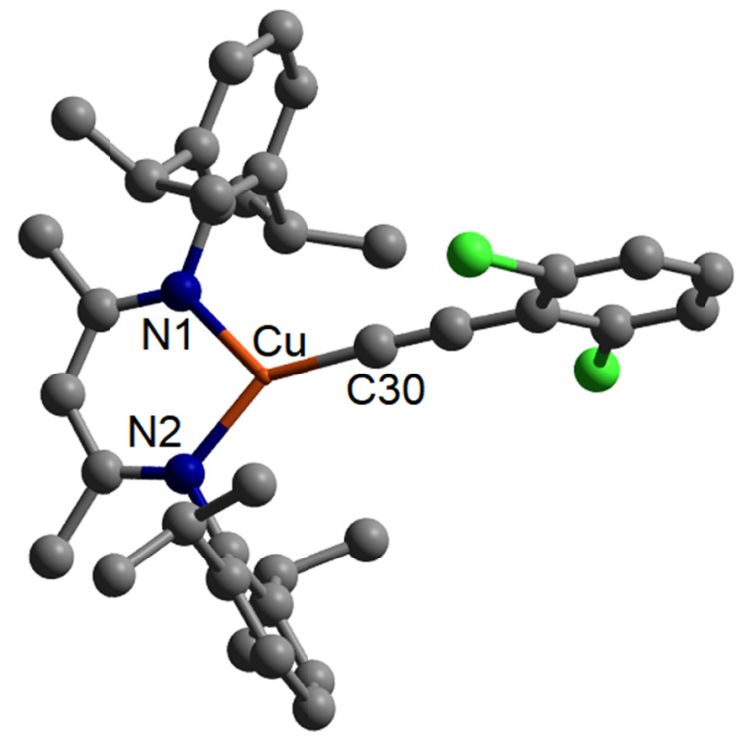

Figure S24. Geometry optimized structure of $\mathrm{T}$-shaped $\left[{ }^{i} \mathrm{Pr}_{2} \mathrm{NN}\right] \mathrm{Cu}-\mathrm{C} \equiv \mathrm{CAr}{ }^{\mathrm{Cl} 2}$ (3) at $\mathrm{BP} 86 / 6-311+\mathrm{G}(\mathrm{d})$ level of theory with charge $=0$, multiplicity $=2$. The bond angles at copper were frozen to T-Shape as obtained from the X-ray crystal structure of $\mathbf{3}$. Selected calculated bond distances $(\AA)$ and angles $\left(^{\circ}\right)$ for 3: Cu1-N1, 1.959; Cu1-N2, 1.917; Cu1C30, 1.873; N1-Cu1-N2, 94.92; N1-Cu1-C30, 22.58; N2-Cu1-C30, 142.51. 


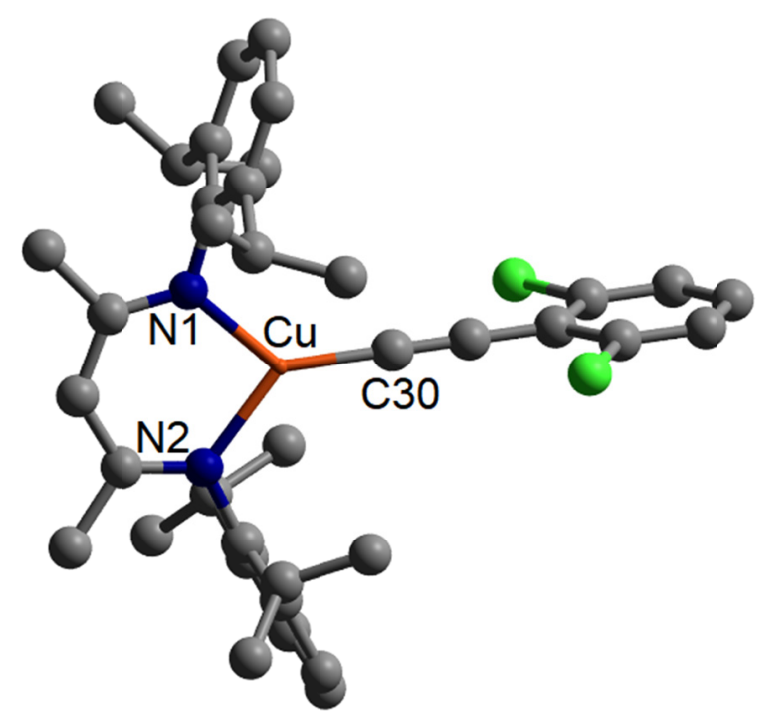

Figure S25. Geometry optimized structure of $\left\{\left[{ }^{i} \mathrm{Pr}_{2} \mathrm{NN}\right] \mathrm{Cu}^{\mathrm{I}}\left(\mathrm{C} \equiv \mathrm{CAr}^{\mathrm{Cl}}\right)\right\}^{-}$(4) at $\mathrm{BP} 86 / 6$ $311+\mathrm{G}(\mathrm{d})$ level of theory with charge $=-1$, multiplicity $=1$. Selected calculated bond distances $(\AA)$ and angles $\left({ }^{\circ}\right)$ for 4 : Cu1-N1, 1.998; Cu1-N2, 1.998; Cu1-C30, 1.854; N1Cu1-N2, 95.23; N1-Cu1-C30, 132.39; N2-Cu1-C30, 132.38.

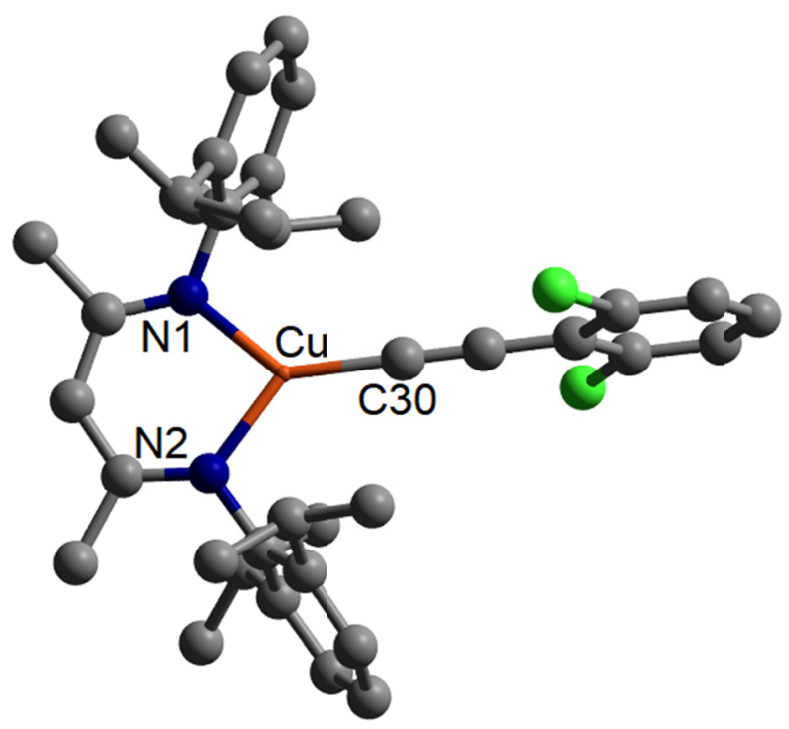

Figure S26. Geometry optimized structure of $\left\{\left[{ }^{i} \mathrm{Pr}_{2} \mathrm{NN}\right] \mathrm{Cu}^{\text {III }}\left(\mathrm{C} \equiv \mathrm{CAr}^{\mathrm{Cl}}\right)\right\}^{+}(\mathbf{8})$ at $\mathrm{BP} 86 / 6-$ $311+G(d)$ level of theory with charge $=0$, multiplicity $=1$. Selected calculated bond distances $(\AA)$ and angles $\left({ }^{\circ}\right)$ for 9: Cu1-N1, 1.920; Cu1-N2, 1.921; Cu1-C30, 1.862; N1Cu1-N2, 95.24; N1-Cu1-C30, 132.66; N2-Cu1-C30, 132.10. 


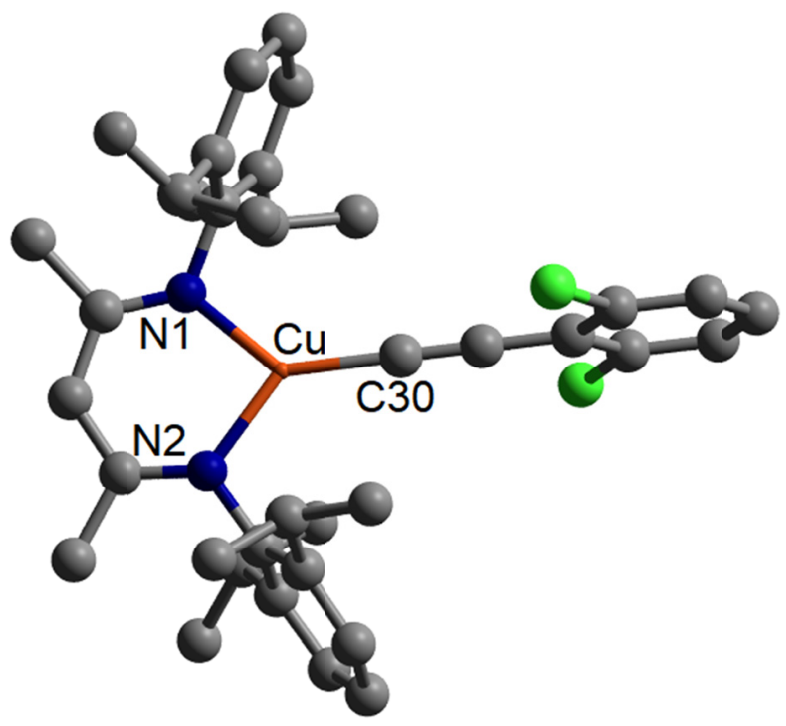

Figure S27. Geometry optimized structure of $\left\{\left[{ }^{i} \mathrm{Pr}_{2} \mathrm{NN}\right] \mathrm{Cu}{ }^{\mathrm{III}}\left(\mathrm{C} \equiv \mathrm{CAr}^{\mathrm{Cl}}\right)\right\}^{+}(8)$ at $\mathrm{BP} 86 / 6$ $311+G(d)$ level of theory with charge $=0$, multiplicity $=3$. Selected calculated bond distances $(\AA)$ and angles $\left({ }^{\circ}\right)$ for 9: Cu1-N1, 1.950; Cu1-N2, 1.950; Cu1-C30, 1.859; N1Cu1-N2, 94.93; N1-Cu1-C30, 132.53; N2-Cu1-C30, 132.54.

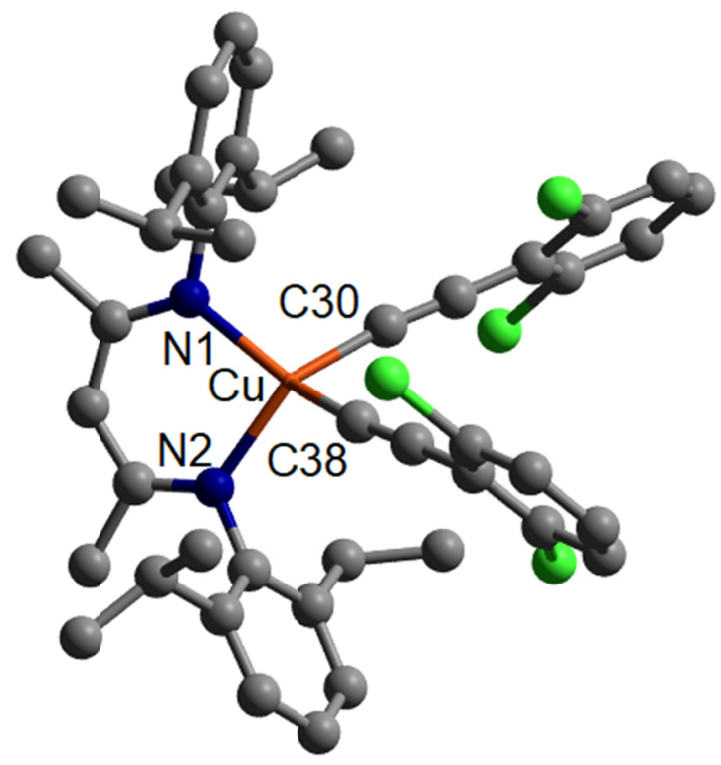

Figure S28. Geometry optimized structure of $\left\{\left[{ }^{i} \mathrm{Pr}_{2} \mathrm{NN}\right] \mathrm{Cu}{ }^{\mathrm{II}}\left(\mathrm{C} \equiv \mathrm{CAr}^{\mathrm{Cl} 2}\right)_{2}\right\}^{-}$at $\mathrm{BP} 86 / 6$ $311+\mathrm{G}(\mathrm{d})$ level of theory with charge $=-1$, multiplicity $=2$. Selected calculated bond distances $(\AA)$ and angles $\left(^{\circ}\right)$ for $\left\{\left[{ }^{i} \operatorname{Pr}_{2} \mathrm{NN}\right] \mathrm{Cu}^{\mathrm{II}}\left(\mathrm{C} \equiv \mathrm{CAr}^{\mathrm{Cl} 2}\right)_{2}\right\}^{-}$: $\mathrm{Cu} 1-\mathrm{N} 1,2.062$; $\mathrm{Cu} 1-\mathrm{N} 2$, 2.052; Cu1-C30, 1.935; Cu1-C38, 1.932; C30-C38, 2.783; N1-Cu1-N2, 92.67; N1-Cu1C30, 101.75; N2-Cu1-C38, 103.30; C30-Cu1-C38, 92.06. 


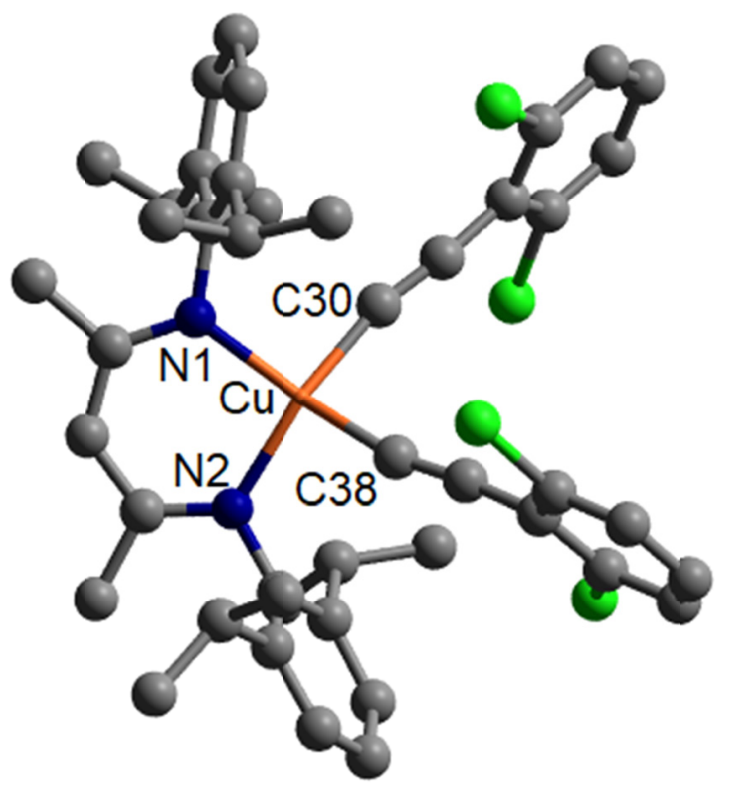

Figure S29. Geometry optimized structure of $\left[{ }^{i} \operatorname{Pr}_{2} N N\right] C \mathrm{Cu}^{\mathrm{III}}\left(\mathrm{C} \equiv \mathrm{CAr}^{\mathrm{Cl2}}\right)(\mathrm{Ph})$ at $\mathrm{BP} 86 / 6$ $311+G(d)$ level of theory with charge $=0$, multiplicity $=1$. Selected calculated bond distances $(\AA)$ and angles $\left({ }^{\circ}\right)$ for $\left[{ }^{i} \mathrm{Pr}_{2} \mathrm{NN}\right] \mathrm{Cu}^{\mathrm{III}}\left(\mathrm{C} \equiv \mathrm{CAr}^{\mathrm{Cl}}\right)(\mathrm{Ph})$ : $\mathrm{Cu} 1-\mathrm{N} 1,1.989$; $\mathrm{Cu} 1-\mathrm{N} 2$, 1.989; Cu1-C30, 1.921; Cu1-C38, 1.921; C30-C38, 2.783; N1-Cu1-N2, 95.28; N1-Cu1C30, 93.07; N2-Cu1-C38, 93.08; C30-Cu1-C38, 92.82.

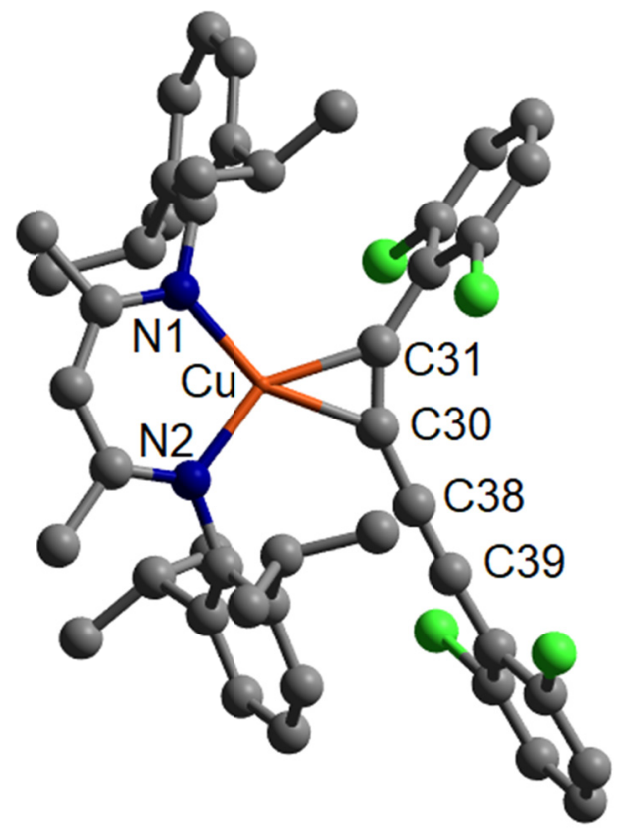

Figure S30. Geometry optimized structure of $\left[{ }^{i} \mathrm{Pr}_{2} \mathrm{NN}\right] \mathrm{Cu}\left(\mathrm{C}^{\mathrm{I}} \mathrm{Cl} \mathrm{ArC} \equiv \mathrm{C}-\mathrm{C} \equiv \mathrm{CAr}{ }^{\mathrm{Cl} 2}\right)$ at $\mathrm{BP} 86 / 6-311+\mathrm{G}(\mathrm{d})$ level of theory with charge $=0$, multiplicity $=1$. Selected calculated 
bond distances $(\AA)$ and angles $\left({ }^{\circ}\right)$ for $\left[{ }^{i} \mathrm{Pr}_{2} \mathrm{NN}\right] \mathrm{Cu}^{\mathrm{I}}\left({ }^{\mathrm{Cl}}{ }^{2} \mathrm{ArC} \equiv \mathrm{C}-\mathrm{C} \equiv \mathrm{CAr}{ }^{\mathrm{Cl} 2}\right)$ : $\mathrm{Cu} 1-\mathrm{N} 1,1.961$; Cu1-N2, 1.955; Cu1-C30, 2.006; Cu1-C31, 1.980; Cu1-C38, 3.148; C30-C38, 1.366; N1Cu1-N2, 98.71; N1-Cu1-C31, 114.56; N2-Cu1-C38, 109.75; C30-Cu1-C31, 37.44; C31C30-C38, 152.67; C30-C38-C39, 176.91.

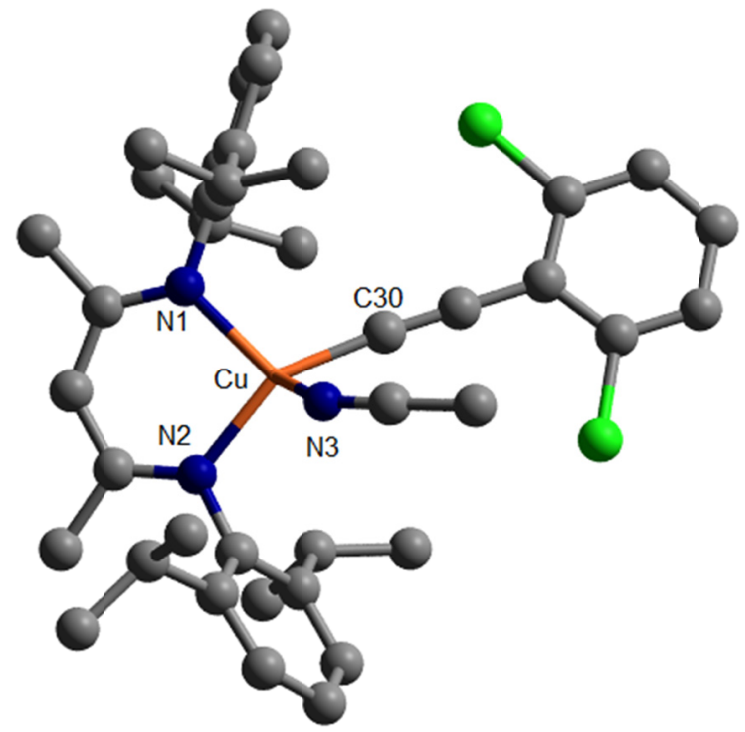

Figure S31. Geometry optimized structure of $\left[{ }^{i} \mathrm{Pr}_{2} \mathrm{NN}\right] C \mathrm{Cu}^{\mathrm{II}}\left(\mathrm{C} \equiv \mathrm{CAr}{ }^{\mathrm{Cl}}\right)(\mathrm{NCMe})$ at $\mathrm{BP} 86 / 6-311+\mathrm{G}(\mathrm{d})$ level of theory with charge $=0$, multiplicity $=2$. Selected calculated bond distances $(\AA)$ and angles $\left({ }^{\circ}\right)$ for $\left[{ }^{i} \operatorname{Pr}_{2} \mathrm{NN}\right] \mathrm{Cu}^{\mathrm{II}}\left(\mathrm{C} \equiv \mathrm{CAr}^{\mathrm{Cl} 2}\right)(\mathrm{NCMe})$ : $\mathrm{Cu} 1-\mathrm{N} 1,2.010$; Cu1-N2，1.964; Cu1-N3，2.304; Cu1-C30，1.910; N1-Cu1-N2，96.01; N1-Cu1-C30, 109.70; N2-Cu1-N3, 97.65; C30-Cu1-C38, 89.32. 


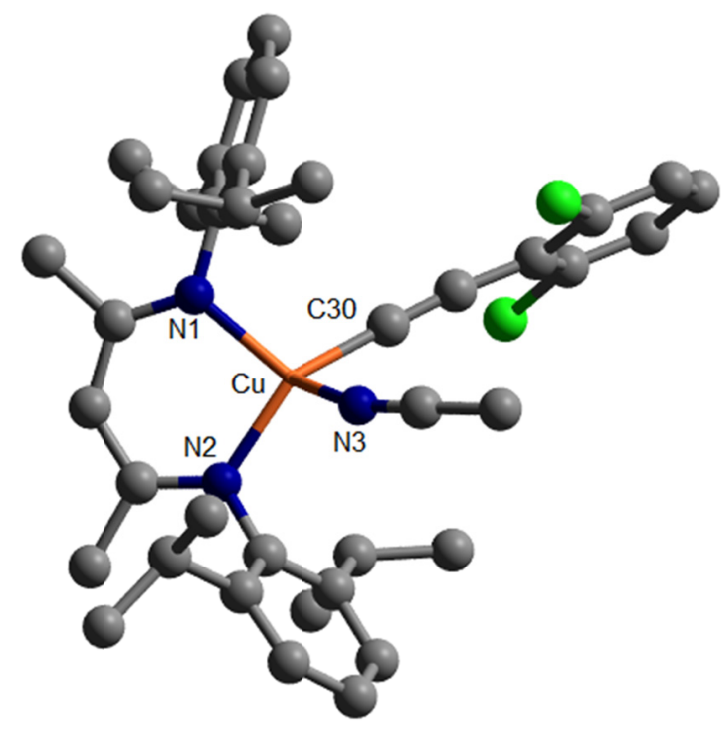

Figure S32. Geometry optimized structure of $\left\{\left[{ }^{i} \mathrm{Pr}_{2} \mathrm{NN}\right] \mathrm{Cu}^{\mathrm{III}}\left(\mathrm{C} \equiv \mathrm{CAr}{ }^{\mathrm{Cl}}\right)(\mathrm{NCMe})\right\}^{+}$at BP86/6-311+G(d) level of theory with charge $=+1$, multiplicity $=1$. Selected calculated bond distances $(\AA)$ and angles $\left(^{\circ}\right)$ for $\left.\left\{{ }^{i} \mathrm{Pr}_{2} \mathrm{NN}\right] \mathrm{Cu}^{\mathrm{III}}\left(\mathrm{C} \equiv \mathrm{CAr}^{\mathrm{Cl}}\right)(\mathrm{NCMe})\right\}^{+}: \mathrm{Cu} 1-\mathrm{N} 1$, 1.992; Cu1-N2, 1.950; Cu1-N3, 2.158; Cu1-C30, 1.894; N1-Cu1-N2, 97.06; N1-Cu1-C30, 107.55 ; N2-Cu1-N3, 98.21; C30-Cu1-C38, 91.07.

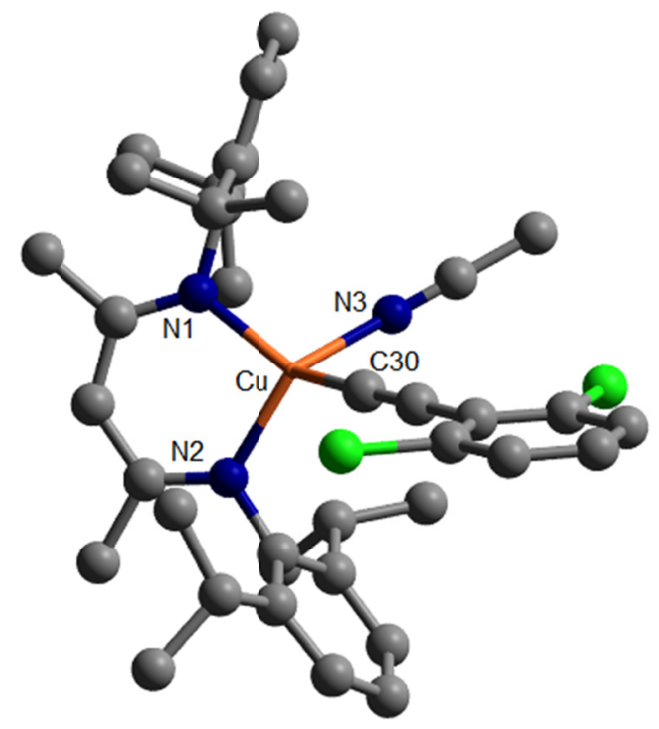

Figure S33. Geometry optimized structure of $\left\{\left[{ }^{i} \mathrm{Pr}_{2} \mathrm{NN}\right] \mathrm{Cu}^{\mathrm{III}}\left(\mathrm{C} \equiv \mathrm{CAr}^{\mathrm{Cl}}\right)(\mathrm{NCMe})\right\}^{+}$at BP86/6-311+G(d) level of theory with charge $=+1$, multiplicity $=3$. Selected calculated bond distances $(\AA)$ and angles $\left(^{\circ}\right)$ for $\left.\left\{{ }^{i} \mathrm{Pr}_{2} \mathrm{NN}\right] \mathrm{Cu}^{\mathrm{III}}\left(\mathrm{C} \equiv \mathrm{CAr}^{\mathrm{Cl}}\right)(\mathrm{NCMe})\right\}^{+}$: $\mathrm{Cu} 1-\mathrm{N} 1$, 1.979; Cu1-N2, 2.002; Cu1-N3, 2.128; Cu1-C30, 1.900; N1-Cu1-N2, 94.85; N2-Cu1-C30, 109.48; N1-Cu1-N3, 99.14; C30-Cu1-C38, 91.07. 


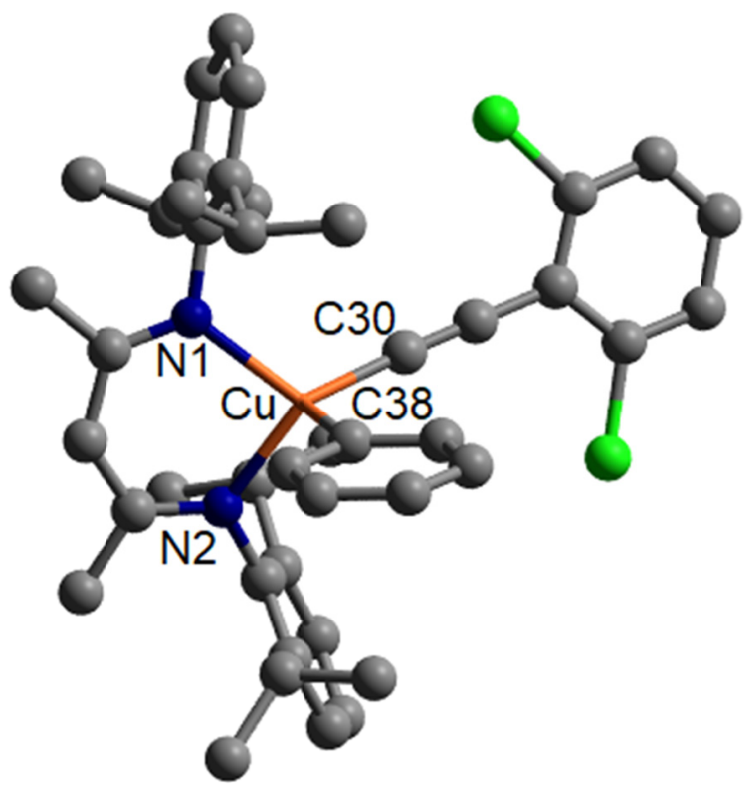

Figure S34. Geometry optimized structure of $\left\{\left[{ }^{i} \mathrm{Pr}_{2} \mathrm{NN}\right] \mathrm{Cu}^{\mathrm{II}}\left(\mathrm{C} \equiv \mathrm{CAr}{ }^{\mathrm{Cl}}\right)(\mathrm{Ph})\right\}^{-}$at $\mathrm{BP} 86 / 6$ $311+\mathrm{G}(\mathrm{d})$ level of theory with charge $=-1$, multiplicity $=2$. Selected calculated bond distances $(\AA)$ and angles $\left({ }^{\circ}\right)$ for $\left.\left\{{ }^{i} \operatorname{Pr}_{2} \mathrm{NN}\right] \mathrm{Cu}^{\mathrm{II}}\left(\mathrm{C} \equiv \mathrm{CAr}{ }^{\mathrm{Cl} 2}\right)(\mathrm{Ph})\right\}^{-}: \mathrm{Cu} 1-\mathrm{N} 1,2.112 ; \mathrm{Cu} 1-\mathrm{N} 2$, 2.059; Cu1-C30, 1.908; Cu1-C38, 2.010; C30-C38, 3.042; N1-Cu1-N2, 92.84; N1-Cu1C30, 117.55; N2-Cu1-C38, 104.11; C30-Cu1-C38, 101.86.

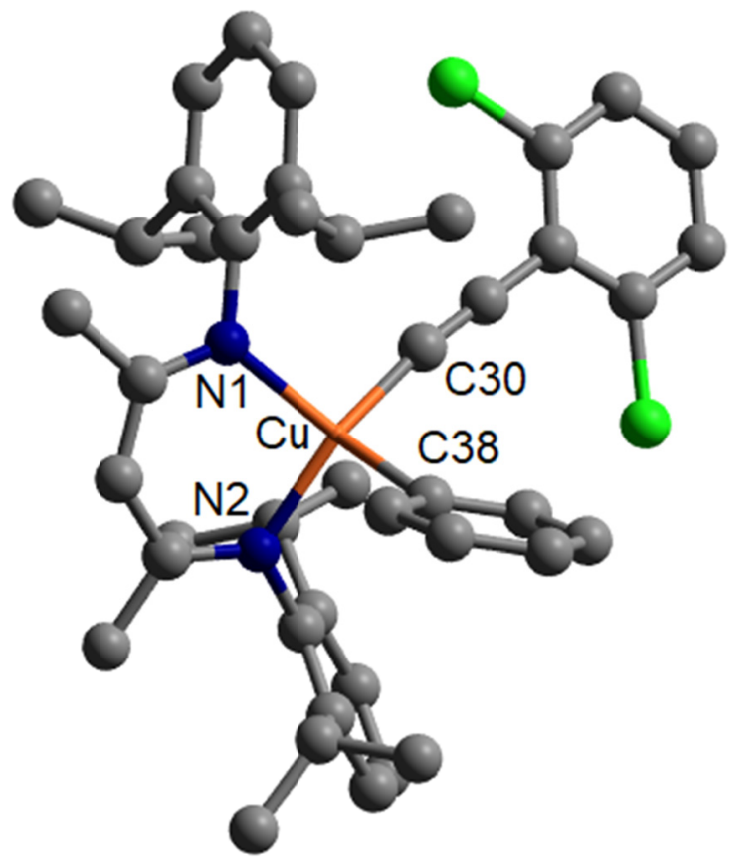

Figure S35. Geometry optimized structure of $\left[{ }^{i} \mathrm{Pr}_{2} \mathrm{NN}\right] \mathrm{Cu}^{\mathrm{III}}\left(\mathrm{C} \equiv \mathrm{CAr}{ }^{\mathrm{Cl} 2}\right)(\mathrm{Ph})$ at $\mathrm{BP} 86 / 6$ $311+\mathrm{G}(\mathrm{d})$ level of theory with charge $=0$, multiplicity $=1$. Selected calculated bond 
distances $(\AA)$ and angles $\left({ }^{\circ}\right)$ for $\left[{ }^{i} \mathrm{Pr}_{2} \mathrm{NN}\right] \mathrm{Cu}^{\mathrm{III}}\left(\mathrm{C} \equiv \mathrm{CAr}^{\mathrm{Cl}}\right)(\mathrm{Ph})$ : $\mathrm{Cu} 1-\mathrm{N} 1,2.042$; $\mathrm{Cu} 1-\mathrm{N} 2$, 1.947; Cu1-C30, 1.917; Cu1-C38, 2.014; C30-C38, 3.042; N1-Cu1-N2, 96.65; N1-Cu1C30, 101.31; N2-Cu1-C38, 92.75; C30-Cu1-C38, 101.36.

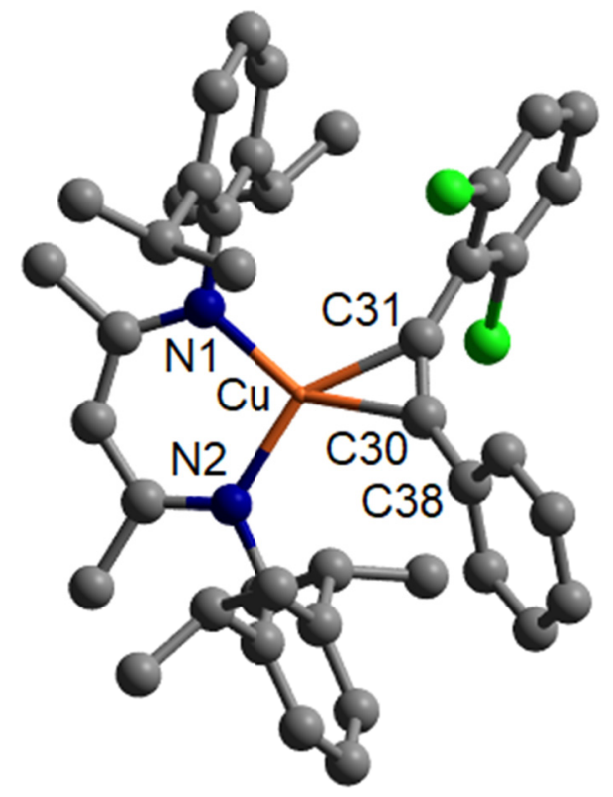

Figure S36. Geometry optimized structure of $\left[{ }^{i} \mathrm{Pr}_{2} \mathrm{NN}\right] \mathrm{Cu}^{\mathrm{I}}\left(\mathrm{C} \equiv \mathrm{CAr}^{\mathrm{Cl}}-\mathrm{Ph}\right)$ at $\mathrm{BP} 86 / 6$ $311+\mathrm{G}(\mathrm{d})$ level of theory with charge $=0$, multiplicity $=1$. Selected calculated bond distances $(\AA)$ and angles $\left({ }^{\circ}\right)$ for $\left[{ }^{i} \mathrm{Pr}_{2} \mathrm{NN}\right] \mathrm{Cu}^{\mathrm{I}}\left(\mathrm{C} \equiv \mathrm{CAr}^{\mathrm{Cl}}-\mathrm{Ph}\right)$ : $\mathrm{Cu} 1-\mathrm{N} 1,1.977$; $\mathrm{Cu} 1-\mathrm{N} 2$, 1.976; Cu1-C30, 2.015; Cu1-C31, 2.003; C30-C38, 1.447; N1-Cu1-N2, 98.19; N1-Cu1C38, 113.57; N2-Cu1-C30, 113.85; C30-Cu1-C31, 36.86; C31-C30-C38; 148.94. 


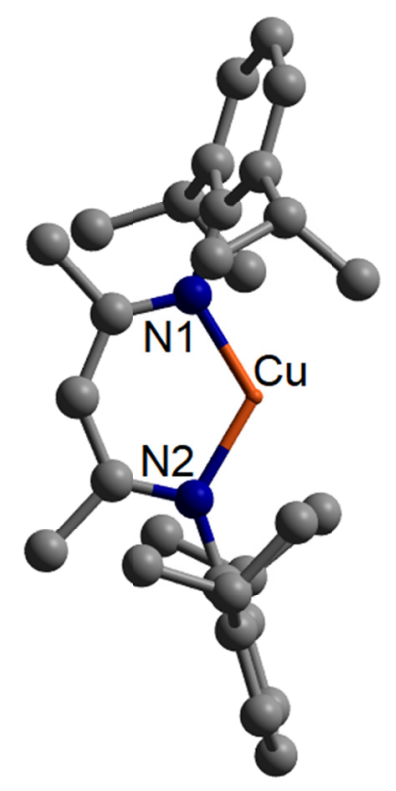

Figure S37. Geometry optimized structure of $\left[{ }^{i} \mathrm{Pr}_{2} \mathrm{NN}\right] \mathrm{Cu}^{\mathrm{I}}(\mathbf{1})$ at $\mathrm{BP} 86 / 6-311+\mathrm{G}(\mathrm{d})$ level of theory with charge $=0$, multiplicity $=1$. Selected calculated bond distances $(\AA)$ and angles $\left({ }^{\circ}\right)$ for $\left\{{ }^{i} \mathrm{Pr}_{2} \mathrm{NN}\right] \mathrm{Cu}^{\mathrm{I}}: \mathrm{Cu} 1-\mathrm{N} 1,1.872 ; \mathrm{Cu} 1-\mathrm{N} 2,1.872 ; \mathrm{N} 1-\mathrm{Cu} 1-\mathrm{N} 2,118.25$.

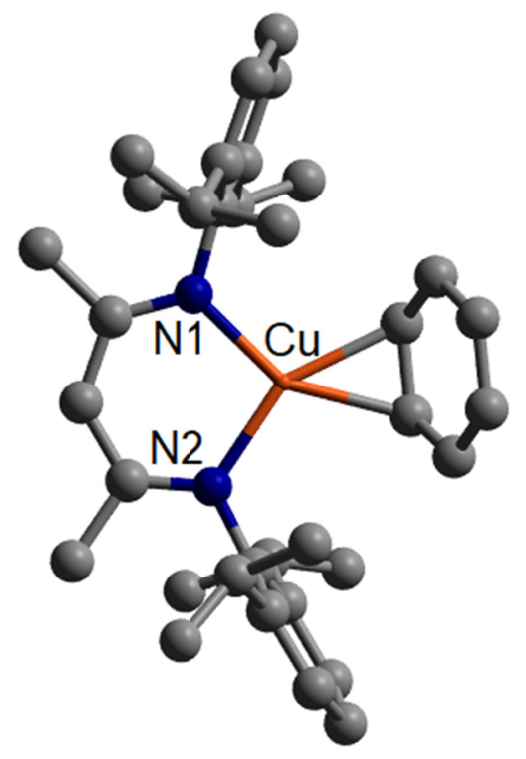

Figure S38. Geometry optimized structure of $\left[{ }^{i} \mathrm{Pr}_{2} \mathrm{NN}\right] \mathrm{Cu}^{\mathrm{I}}\left(\eta^{2}-\mathrm{C}_{6} \mathrm{H}_{6}\right)\left(\mathbf{1}-\mathrm{C}_{\mathbf{6}} \mathbf{H}_{6}\right)$ at BP86/6$311+\mathrm{G}(\mathrm{d})$ level of theory with charge $=0$, multiplicity $=1$. Selected calculated bond distances $(\AA)$ and angles $\left(^{\circ}\right)$ for $\left\{\left[{ }^{i} \mathrm{Pr}_{2} \mathrm{NN}\right] \mathrm{Cu}^{\mathrm{I}}\left(\eta^{2}-\mathrm{C}_{6} \mathrm{H}_{6}\right)\right.$ : Cu1-N1, 1.963; Cu1-N2, 1.963; N1-Cu1-N2, 100.76. 


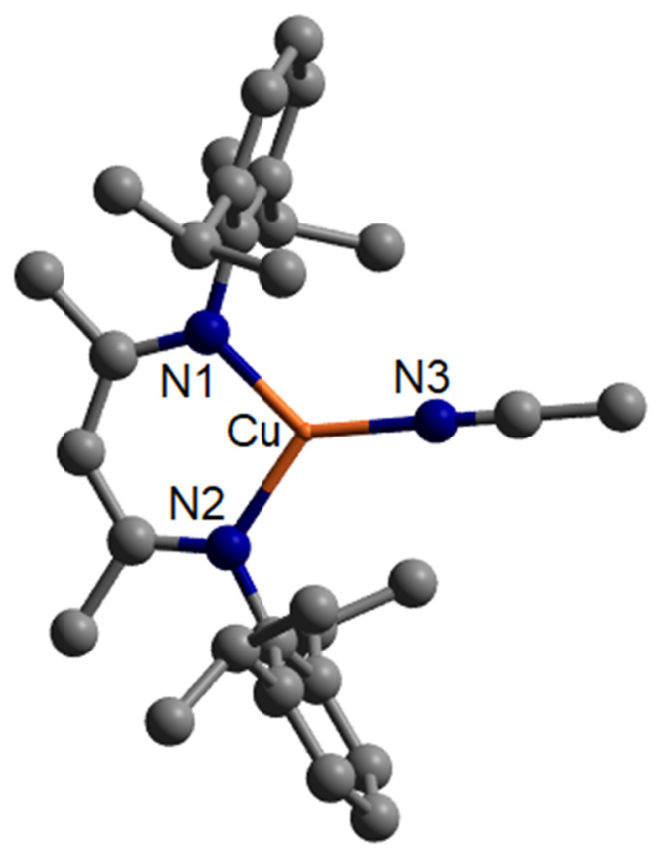

Figure S39. Geometry optimized structure of $\left[{ }^{i} \mathrm{Pr}_{2} \mathrm{NN}\right] \mathrm{Cu}^{\mathrm{I}}(\mathrm{NCMe})(\mathbf{1 - N C M e})$ at $\mathrm{BP} 86 / 6$ $311+\mathrm{G}(\mathrm{d})$ level of theory with charge $=0$, multiplicity $=1$. Selected calculated bond distances $(\AA)$ and angles $\left({ }^{\circ}\right)$ for $\left\{\left[{ }^{i} \mathrm{Pr}_{2} \mathrm{NN}\right] \mathrm{Cu}^{\mathrm{I}}(\mathrm{NCMe})\right.$ : Cu1-N1, 1.951; Cu1-N2, 1.952; Cu1-N3: 1.849; N1-Cu1-N2, 99.92; N1-Cu1-N3: 130.35; N2-Cu1-N3: 129.74. 


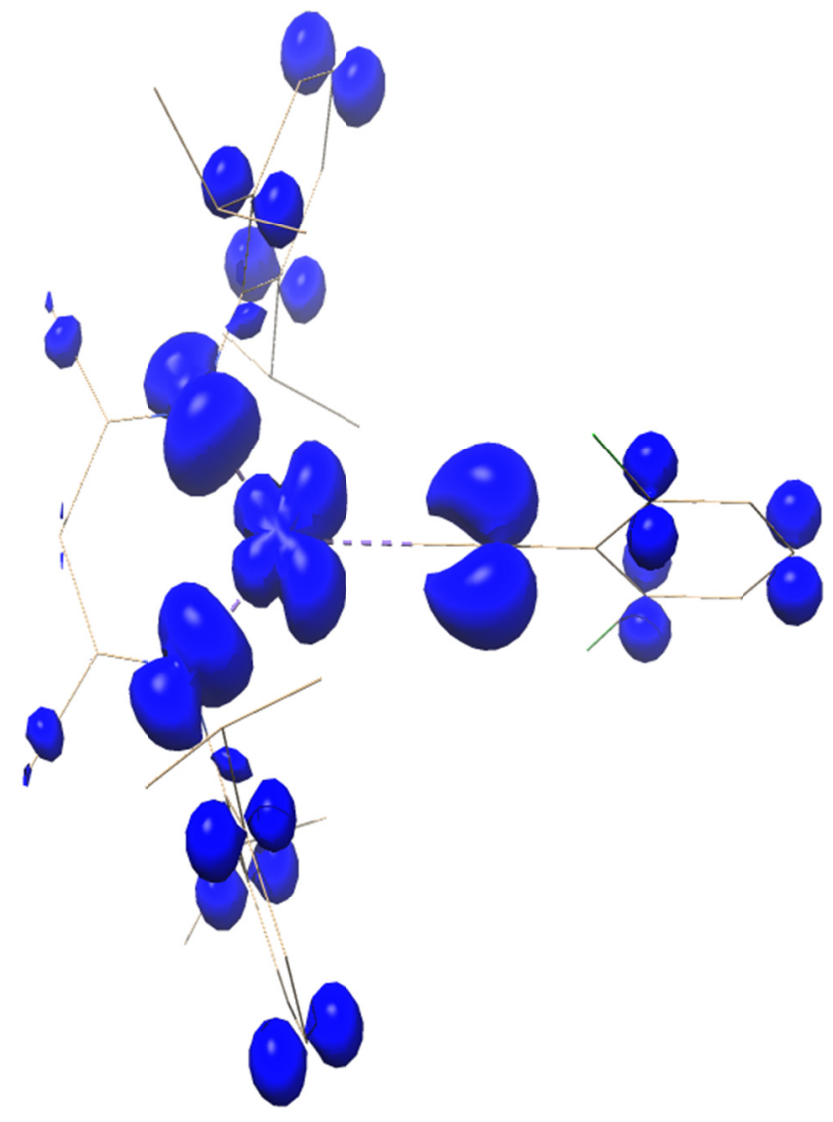

Figure S40. Total spin density plot of $\left[{ }^{i} \mathrm{Pr}_{2} \mathrm{NN}\right] \mathrm{Cu}-\mathrm{C} \equiv \mathrm{CAr}^{\mathrm{Cl} 2}$ (3) (Y-shaped) with an isosurface of 0.002. Predicted spin densities (e): Cu1, 0.378; N1, 0.153; N2, 0.153; C30, $-0.030 ; \mathrm{C} 31,0.122 ;\left(\mathrm{Ar}^{\mathrm{Cl} 2}\right)_{\text {total }}, 0.059$. 
Table S9. Thermodynamics of the model reactions using BP86/6-311+G(d) with benzene solvent and GD3BJ dispersion correction included. All values are in $\mathrm{kcal} / \mathrm{mol}$ at $298.15 \mathrm{~K}$.

\begin{tabular}{|c|c|c|c|}
\hline & & \begin{tabular}{|l} 
Enthalpy \\
\end{tabular} & \begin{tabular}{|l|} 
Free Energy \\
\end{tabular} \\
\hline 1 & {$\left[{ }^{i} \mathrm{Pr}_{2} \mathrm{NN}\right] \mathrm{Cu}-\mathrm{C} \equiv \mathrm{CAr}{ }^{\mathrm{Cl} 2} \rightarrow\left[{ }^{i} \mathrm{Pr}_{2} \mathrm{NN}\right] \mathrm{Cu}+{ }^{\mathrm{Cl} 2} \mathrm{ArC} \equiv \mathrm{C} \cdot$ radical } & +84.7 & +73.1 \\
\hline $2 *$ & $2\left[{ }^{i} \mathrm{Pr}_{2} \mathrm{NN}\right] \mathrm{Cu}-\mathrm{C} \equiv \mathrm{CAr}^{\mathrm{Cl} 2} \rightarrow\left\{\left[\mathrm{Cu}^{\mathrm{III}}\right]-\mathrm{C} \equiv \mathrm{CAr}^{\mathrm{Cl} 2}\right\}^{+}+\left\{\left[\mathrm{Cu}^{\mathrm{I}}\right]-\mathrm{C} \equiv \mathrm{CAr}^{\mathrm{Cl} 2}\right\}^{-}$ & +44.5 & +47.0 \\
\hline $3 *$ & $\left\{\left[\mathrm{Cu}^{\mathrm{III}}\right]-\mathrm{C} \equiv \mathrm{CAr}^{\mathrm{Cl} 12}\right\}^{+}+\left\{\left[\mathrm{Cu}^{\mathrm{l}}\right]-\mathrm{C} \equiv \mathrm{CAr}^{\mathrm{Cl} 2}\right\}^{-} \rightarrow\left[\mathrm{Cu}^{\mathrm{III}}\right]\left(\mathrm{C} \equiv \mathrm{CAr}^{\mathrm{Cl} 2}\right)_{2}+\left[\mathrm{Cu}^{\mathrm{I}}\right]$ & -16.4 & -13.5 \\
\hline 4 & $\left\{\left[\mathrm{Cu}^{\mathrm{III}}\right]-\mathrm{C} \equiv \mathrm{CAr}^{\mathrm{Cl} 2}\right\}^{+}+\left\{\left[\mathrm{Cu}^{\mathrm{I}}\right]-\mathrm{C} \equiv \mathrm{CAr}^{\mathrm{Cl} 2}\right\}^{-}+\mathrm{C}_{6} \mathrm{H}_{\mathrm{C}} \rightarrow\left[\mathrm{Cu}^{\mathrm{III}}\right]\left(\mathrm{C} \equiv \mathrm{CAr}^{\mathrm{Cl} 2}\right)_{2}+\left[\mathrm{Cu}^{\mathrm{I}}\right]\left(\eta^{2}-\mathrm{C}_{6} \mathrm{H}_{6}\right)$ & -42.7 & -25.4 \\
\hline 5* & {$\left[{ }^{i} \mathrm{Pr}_{2} \mathrm{NN}\right] \mathrm{Cu}-\mathrm{C} \equiv \mathrm{CAr}^{\mathrm{Cl} 2}+{ }^{\mathrm{Cl} 2} \mathrm{ArC} \equiv \mathrm{C}^{-} \rightarrow\left\{\left[\mathrm{Cu}^{\mathrm{II}}\right]\left(\mathrm{C} \equiv \mathrm{CAr}^{\mathrm{Cl} 2}\right)_{2}\right\}^{-}$} & -30.7 & -15.8 \\
\hline $6^{*}$ & $\left\{\left[\mathrm{Cu}^{\mathrm{II}}\right]\left(\mathrm{C} \equiv \mathrm{CAr}^{\mathrm{Cl} 2}\right)_{2}\right\}^{-}+\left[{ }^{i} \mathrm{Pr}_{2} \mathrm{NN}\right] \mathrm{Cu}-\mathrm{C} \equiv \mathrm{CAr}^{\mathrm{Cl} 2} \rightarrow\left[\mathrm{Cu}^{\mathrm{III}}\right]\left(\mathrm{C} \equiv \mathrm{CAr}^{\mathrm{Cl} 2}\right)_{2}+\left\{\left[\mathrm{Cu}^{\mathrm{I}}\right]-\mathrm{C} \equiv \mathrm{CAr}^{\mathrm{Cl} 2}\right\}^{-}$ & +1.2 & +3.9 \\
\hline 7 & {$\left[\mathrm{Cu}^{\mathrm{III}}\right]\left(\mathrm{C} \equiv \mathrm{CAr}^{\mathrm{Cl} 2}\right)_{2} \rightarrow\left[\mathrm{Cu}^{\mathrm{I}}\right]\left({ }^{\mathrm{Cl} 2} \mathrm{ArC} \equiv \mathrm{C}-\mathrm{C} \equiv \mathrm{CAr}^{\mathrm{Cl} 2}\right)$} & -55.4 & -53.4 \\
\hline 8 & {$\left[\mathrm{Cu}^{\mathrm{I}}\right]\left({ }^{\mathrm{Cl} 2} \mathrm{ArC} \equiv \mathrm{C}-\mathrm{C} \equiv \mathrm{CAr}{ }^{\mathrm{Cl} 2}\right) \rightarrow\left[\mathrm{Cu}^{\mathrm{I}}\right]+{ }^{\mathrm{Cl} 2} \mathrm{ArC} \equiv \mathrm{C}-\mathrm{C} \equiv \mathrm{CAr}{ }^{\mathrm{Cl} 2}$} & +40.1 & +21.7 \\
\hline 9 & {$\left[\mathrm{Cu}^{\mathrm{I}}\right]\left({ }^{\mathrm{Cl} 2} \mathrm{ArC} \equiv \mathrm{C}-\mathrm{C} \equiv \mathrm{CAr}{ }^{\mathrm{Cl} 2}\right)+\mathrm{C}_{6} \mathrm{H}_{6} \rightarrow\left[\mathrm{Cu}^{\mathrm{I}}\right]\left(\eta^{2}-\mathrm{C}_{6} \mathrm{H}_{6}\right)+{ }^{\mathrm{Cl} 2} \mathrm{ArC} \equiv \mathrm{C}-\mathrm{C} \equiv \mathrm{CAr}{ }^{\mathrm{Cl} 2}$} & +13.9 & +9.7 \\
\hline 10 & $2\left[{ }^{i} \mathrm{Pr}_{2} \mathrm{NN}\right] \mathrm{Cu}-\mathrm{C} \equiv \mathrm{CAr}^{\mathrm{Cl} 12}+2 \mathrm{C}_{6} \mathrm{H}_{6} \rightarrow 2\left[\mathrm{Cu}^{\mathrm{I}}\right]\left(\eta^{2}-\mathrm{C}_{6} \mathrm{H}_{6}\right)+{ }^{\mathrm{C} 12} \mathrm{ArC} \equiv \mathrm{C}-\mathrm{C} \equiv \mathrm{CAr}{ }^{\mathrm{Cl} 2}$ & -39.6 & -22.1 \\
\hline 11 & {$\left[{ }^{i} \mathrm{Pr}_{2} \mathrm{NN}\right] \mathrm{Cu}-\mathrm{C} \equiv \mathrm{CAr}{ }^{\mathrm{Cl} 2}+\mathrm{NCMe} \rightarrow\left\{\left[\mathrm{Cu}^{\mathrm{II}}\right]\left(\mathrm{C} \equiv \mathrm{CAr}^{\mathrm{Cl} 2}\right)(\mathrm{NCMe})\right.$} & -3.4 & +8.5 \\
\hline 12 & {$\left[\mathrm{Cu}^{\mathrm{II}}\right]\left(\mathrm{C} \equiv \mathrm{CAr}{ }^{\mathrm{Cl} 2}\right)(\mathrm{NCMe})+\left[{ }^{i} \mathrm{Pr}_{2} \mathrm{NN}\right] \mathrm{Cu}-\mathrm{C} \equiv \mathrm{CAr}^{\mathrm{Cl} 2} \rightarrow\left\{\left[\mathrm{Cu}^{\mathrm{III}}\right]\left(\mathrm{C} \equiv \mathrm{CAr}^{\mathrm{Cl} 2}\right)(\mathrm{NCMe})\right\}^{+}+\left\{\left[\mathrm{Cu}^{\mathrm{I}}\right]-\mathrm{C} \equiv \mathrm{CAr}{ }^{\mathrm{Cl} 2}\right\}$} & +42.1 & +44.6 \\
\hline 13 & $2\left[{ }^{i} \mathrm{Pr}_{2} \mathrm{NN}\right] \mathrm{Cu}-\mathrm{C} \equiv \mathrm{CAr}^{\mathrm{Cl} 2}+{ }^{\mathrm{Cl} 2} \mathrm{ArC} \equiv \mathrm{C}^{-} \rightarrow\left\{\left[\mathrm{Cu}^{\mathrm{I}}\right]-\mathrm{C} \equiv \mathrm{CAr}^{\mathrm{Cl} 2}\right\}^{-}+\left[\mathrm{Cu}^{\mathrm{I}}\right]\left(\eta^{2}-\mathrm{C}_{6} \mathrm{H}_{6}\right)+{ }^{\mathrm{Cl} 2} \mathrm{ArC} \equiv \mathrm{C}-\mathrm{C} \equiv \mathrm{CAr}{ }^{\mathrm{Cl} 2}$ & -71.0 & -55.6 \\
\hline 14 & {$\left[{ }^{i} \mathrm{Pr}_{2} \mathrm{NN}\right] \mathrm{Cu}-\mathrm{C} \equiv \mathrm{CAr}^{\mathrm{Cl} 2}+\mathrm{Ph}^{-} \rightarrow\left\{\left[\mathrm{Cu}^{\mathrm{II}}\right]\left(\mathrm{C} \equiv \mathrm{CAr}^{\mathrm{Cl} 2}\right)(\mathrm{Ph})\right\}^{-}$} & -48.7 & -35.9 \\
\hline $15^{* *}$ & $\left\{\left[\mathrm{Cu}^{\mathrm{II}}\right]\left(\mathrm{C} \equiv \mathrm{CAr}^{\mathrm{Cl}}\right)(\mathrm{Ph})\right\}^{-}+\left[{ }^{i} \mathrm{Pr}_{2} \mathrm{NN}\right] \mathrm{Cu}-\mathrm{C} \equiv \mathrm{CAr}^{\mathrm{Cl} 2} \rightarrow\left[\mathrm{Cu}^{\mathrm{III}}\right]\left(\mathrm{C} \equiv \mathrm{CAr}{ }^{\mathrm{Cl} 2}\right)(\mathrm{Ph})+\left\{\left[\mathrm{Cu}^{\mathrm{I}}\right]-\mathrm{C} \equiv \mathrm{CAr}^{\mathrm{Cl} 2}\right\}^{-}$ & -5.8 & -0.7 \\
\hline $16^{* *}$ & {$\left[\mathrm{Cu}^{\mathrm{III}}\right]\left(\mathrm{C} \equiv \mathrm{CAr}{ }^{\mathrm{Cl} 2}\right)(\mathrm{Ph}) \rightarrow\left[\mathrm{Cu}^{\mathrm{I}}\right]\left({ }^{\mathrm{Cl} 2} \mathrm{ArC} \equiv \mathrm{C}-\mathrm{Ph}\right)$} & -56.1 & -53.9 \\
\hline 17 & {$\left[\mathrm{Cu}^{\mathrm{I}}\right]\left(\mathrm{C}^{\mathrm{Cl}} \mathrm{ArC} \equiv \mathrm{C}-\mathrm{Ph}\right) \rightarrow\left[\mathrm{Cu}^{\mathrm{I}}\right]+{ }^{\mathrm{Cl} 2} \mathrm{ArC} \equiv \mathrm{C}-\mathrm{Ph}$} & +39.0 & +20.4 \\
\hline 18 & {$\left[\mathrm{Cu}^{\mathrm{I}}\right]\left({ }^{\mathrm{Cl} 2} \mathrm{ArC} \equiv \mathrm{C}-\mathrm{Ph}\right)+\mathrm{C}_{6} \mathrm{H}_{6} \rightarrow\left[\mathrm{Cu}^{\mathrm{I}}\right]\left(\eta^{2}-\mathrm{C}_{6} \mathrm{H}_{6}\right)+{ }^{\mathrm{Cl} 2} \mathrm{ArC} \equiv \mathrm{C}-\mathrm{Ph}$} & +12.8 & +8.5 \\
\hline 19 & $2\left[{ }^{i} \mathrm{Pr}_{2} \mathrm{NN}\right] \mathrm{Cu}-\mathrm{C} \equiv \mathrm{CAr}^{\mathrm{Cl} 2}+\mathrm{Ph}^{-} \rightarrow\left\{\left[\mathrm{Cu}^{1}\right]-\mathrm{C} \equiv \mathrm{CAr}^{\mathrm{Cl} 2}\right\}^{-}+\left[\mathrm{Cu}^{\mathrm{I}}\right]\left(\eta^{2}-\mathrm{C}_{6} \mathrm{H}_{6}\right)+{ }^{\mathrm{Cl} 2} \mathrm{ArC} \equiv \mathrm{C}-\mathrm{Ph}$ & -97.8 & -82.0 \\
\hline
\end{tabular}

A constrained (C30-C38 distances fixed to $2.783 \AA$ A) singlet $\left[{ }^{i} \mathrm{Pr}_{2} \mathrm{NN}\right] \mathrm{Cu} u^{I I I}\left(\mathrm{C} \equiv \mathrm{CAr}^{\mathrm{Cl} 2}\right)_{2}$, ( charge $=0$, multiplicity $\left.=1\right)$ has been employed as an estimate to calculate the thermodynamics of model reactions marked with (*). A constrained (C30-C38 distances fixed to $3.042 \mathrm{~A})$ singlet $\left.{ }^{\mathrm{i}} \mathrm{Pr}_{2} \mathrm{NN}\right] \mathrm{Cu}^{I I I}\left(\mathrm{C} \equiv \mathrm{CAr}{ }^{\mathrm{Cl}}\right)(\mathrm{Ph})$, (charge $=0$, multiplicity $\left.=1\right)$ has been employed as an estimate to calculate the thermodynamics of model reactions marked with (**). 
Table S10. Thermodynamics of the model reactions using BP86/6-311+G(d) with acetonitrile solvent and GD3BJ dispersion correction included. All values are in $\mathrm{kcal} / \mathrm{mol}$ at $298.15 \mathrm{~K}$.

\begin{tabular}{|c|c|c|c|}
\hline & & Enthalpy & Free Energy \\
\hline 1 & {$\left[{ }^{i} \mathrm{Pr}_{2} \mathrm{NN}\right] \mathrm{Cu}-\mathrm{C} \equiv \mathrm{CAr}{ }^{\mathrm{Cl} 2} \rightarrow\left[{ }^{i} \mathrm{Pr}_{2} \mathrm{NN}\right] \mathrm{Cu}+{ }^{\mathrm{Cl} 2} \mathrm{ArC} \equiv \mathrm{C} \cdot$ radical } & +81.5 & +69.9 \\
\hline 2 & $2\left[{ }^{i} \mathrm{Pr}_{2} \mathrm{NN}\right] \mathrm{Cu}-\mathrm{C} \equiv \mathrm{CAr}^{\mathrm{Cl} 2} \rightarrow\left\{\left[\mathrm{Cu}^{\mathrm{III}}\right]-\mathrm{C} \equiv \mathrm{CAr}^{\mathrm{Cl} 2}\right\}^{+}+\left\{\left[\mathrm{Cu}^{\mathrm{I}}\right]-\mathrm{C} \equiv \mathrm{CAr}^{\mathrm{Cl} 2}\right\}^{-}$ & +19.3 & +21.7 \\
\hline 3* & $\left\{\left[\mathrm{Cu}^{\mathrm{III}}\right]-\mathrm{C} \equiv \mathrm{CAr}^{\mathrm{Cl} 2}\right\}^{+}+\left\{\left[\mathrm{Cu}^{\mathrm{I}}\right]-\mathrm{C} \equiv \mathrm{CAr}^{\mathrm{Cl} 2}\right\}^{-} \rightarrow\left[\mathrm{Cu}^{\mathrm{III}}\right]\left(\mathrm{C} \equiv \mathrm{CAr}^{\mathrm{Cl} 2}\right)_{2}+\left[\mathrm{Cu}^{\mathrm{I}}\right]$ & +8.1 & +11.0 \\
\hline 4* & $\left\{\left[\mathrm{Cu}^{\mathrm{III}}\right]-\mathrm{C} \equiv \mathrm{CAr}^{\mathrm{Cl} 2}\right\}^{+}+\left\{\left[\mathrm{Cu}^{\mathrm{I}}\right]-\mathrm{C} \equiv \mathrm{CAr}^{\mathrm{Cl} 2}\right\}^{-}+\mathrm{MeCN} \rightarrow\left[\mathrm{Cu}^{\mathrm{III}}\right]\left(\mathrm{C} \equiv \mathrm{CAr}^{\mathrm{Cl} 2}\right)_{2}+\left[\mathrm{Cu}^{\mathrm{I}}\right](\mathrm{NCMe})$ & -14.3 & -2.3 \\
\hline 5 & {$\left[{ }^{i} \mathrm{Pr}_{2} \mathrm{NN}\right] \mathrm{Cu}-\mathrm{C} \equiv \mathrm{CAr}^{\mathrm{Cl} 2}+{ }^{\mathrm{Cl} 2} \mathrm{ArC} \equiv \mathrm{C}^{-} \rightarrow\left\{\left[\mathrm{Cu}^{\mathrm{II}}\right]\left(\mathrm{C} \equiv \mathrm{CAr}^{\mathrm{C} 2}\right)_{2}\right\}^{-}$} & -19.7 & -4.8 \\
\hline 6* & $\left\{\left[\mathrm{Cu}^{\mathrm{II}}\right]\left(\mathrm{C} \equiv \mathrm{CAr}^{\mathrm{Cl} 2}\right)_{2}\right\}^{-}+\left[{ }^{i} \mathrm{Pr}_{2} \mathrm{NN}\right] \mathrm{Cu}-\mathrm{C} \equiv \mathrm{CAr}^{\mathrm{Cl} 2} \rightarrow\left[\mathrm{Cu}^{\mathrm{III}}\right]\left(\mathrm{C} \equiv \mathrm{CAr}^{\mathrm{Cl} 2}\right)_{2}+\left\{\left[\mathrm{Cu}^{\mathrm{I}}\right]-\mathrm{C} \equiv \mathrm{CAr}^{\mathrm{Cl} 2}\right\}^{-}$ & +1.3 & +3.9 \\
\hline $7 *$ & {$\left[\mathrm{Cu}^{\mathrm{II}}\right]\left(\mathrm{C} \equiv \mathrm{CAr}^{\mathrm{Cl} 2}\right)_{2} \rightarrow\left[\mathrm{Cu}^{\mathrm{I}}\right]\left({ }^{\mathrm{Cl} 2} \mathrm{ArC} \equiv \mathrm{C}-\mathrm{C} \equiv \mathrm{CAr}^{\mathrm{Cl} 2}\right)$} & -51.3 & -49.4 \\
\hline 8 & {$\left[\mathrm{Cu}^{\mathrm{I}}\right]\left({ }^{\mathrm{Cl} 2} \mathrm{ArC} \equiv \mathrm{C}-\mathrm{C} \equiv \mathrm{CAr}{ }^{\mathrm{Cl} 2}\right) \rightarrow\left[\mathrm{Cu}^{\mathrm{I}}\right]+{ }^{\mathrm{Cl} 2} \mathrm{ArC} \equiv \mathrm{C}-\mathrm{C} \equiv \mathrm{CAr}{ }^{\mathrm{Cl} 2}$} & +38.8 & +20.4 \\
\hline 9 & $\left.\left[\mathrm{Cu}^{\mathrm{l}}\right]{ }^{\mathrm{Cl} 2} \mathrm{ArC} \equiv \mathrm{C}-\mathrm{C} \equiv \mathrm{CAr}{ }^{\mathrm{Cl} 2}\right)+\mathrm{MeCN} \rightarrow\left[\mathrm{Cu}^{\mathrm{l}}\right](\mathrm{NCMe})+{ }^{\mathrm{Cl} 2} \mathrm{ArC} \equiv \mathrm{C}-\mathrm{C} \equiv \mathrm{CAr}{ }^{\mathrm{Cl} 2}$ & +16.3 & +7.0 \\
\hline 10 & $2\left[{ }^{i} \mathrm{Pr}_{2} \mathrm{NN}\right] \mathrm{Cu}-\mathrm{C} \equiv \mathrm{CAr}{ }^{\mathrm{Cl} 2}+2 \mathrm{MeCN} \rightarrow 2\left[\mathrm{Cu}^{\mathrm{I}}\right](\mathrm{NCMe})+{ }^{\mathrm{Cl} 2} \mathrm{ArC} \equiv \mathrm{C}-\mathrm{C} \equiv \mathrm{CAr}{ }^{\mathrm{Cl} 2}$ & -30.0 & -23.0 \\
\hline 11 & {$\left[{ }^{i} \mathrm{Pr}_{2} \mathrm{NN}\right] \mathrm{Cu}-\mathrm{C} \equiv \mathrm{CAr}{ }^{\mathrm{Cl} 2}+\mathrm{NCMe} \rightarrow\left\{\left[\mathrm{Cu}^{\mathrm{Il}}\right]\left(\mathrm{C} \equiv \mathrm{CAr}{ }^{\mathrm{Cl} 2}\right)(\mathrm{NCMe})\right.$} & -0.3 & +11.5 \\
\hline 12 & {$\left[\mathrm{Cu}^{\mathrm{II}}\right]\left(\mathrm{C} \equiv \mathrm{CAr}{ }^{\mathrm{Cl} 2}\right)(\mathrm{NCMe})+\left[{ }^{i} \mathrm{Pr}_{2} \mathrm{NN}\right] \mathrm{Cu}-\mathrm{C} \equiv \mathrm{CAr}^{\mathrm{Cl} 2} \rightarrow\left\{\left[\mathrm{Cu}^{\mathrm{III}}\right]\left(\mathrm{C} \equiv \mathrm{CAr}^{\mathrm{Cl} 2}\right)(\mathrm{NCMe})\right\}^{+}+\left\{\left[\mathrm{Cu}^{\mathrm{I}}\right]-\mathrm{C} \equiv \mathrm{CAr}^{\mathrm{Cl} 2}\right\}^{-}$} & +18.1 & +20.6 \\
\hline
\end{tabular}

$A$ constrained (C30-C38 distances fixed to $2.783 \mathrm{~A})$ singlet $\left[{ }^{i} \mathrm{Pr}_{2} \mathrm{NN}\right] \mathrm{Cu} u^{I I I}\left(\mathrm{C} \equiv \mathrm{CAr}^{\mathrm{Cl2}}\right)_{2}$, (charge $=0$, multiplicity $\left.=1\right)$ has been employed as an estimate to calculate the thermodynamics of model reactions marked with (*). 
Table S11. Geometry optimized structure of $\mathrm{Y}$-shaped $\left[{ }^{i} \mathrm{Pr}_{2} \mathrm{NN}\right] \mathrm{Cu}-\mathrm{C} \equiv \mathrm{CAr}{ }^{\mathrm{Cl} 2}$ (3) at BP86/6-311+G(d) level of theory.

$\begin{array}{lrrr}\mathrm{Cu} & 0.671549000 & -0.000205000 & -0.000005000 \\ \mathrm{~N} & 1.977200000 & -1.423687000 & -0.114366000 \\ \mathrm{~N} & 1.978358000 & 1.422207000 & 0.114369000 \\ \mathrm{C} & 3.305809000 & -1.263355000 & -0.079224000 \\ \mathrm{C} & 3.930353000 & -0.001533000 & 0.000001000 \\ \mathrm{H} & 5.021543000 & -0.001977000 & 0.000002000 \\ \mathrm{C} & 3.306836000 & 1.260797000 & 0.079224000 \\ \mathrm{C} & 4.196914000 & -2.490320000 & -0.124600000 \\ \mathrm{H} & 5.255187000 & -2.208178000 & -0.210621000 \\ \mathrm{H} & 3.932538000 & -3.141428000 & -0.972657000 \\ \mathrm{H} & 4.073919000 & -3.098746000 & 0.786397000 \\ \mathrm{C} & 4.198947000 & 2.487033000 & 0.124554000 \\ \mathrm{H} & 5.256960000 & 2.204028000 & 0.210929000 \\ \mathrm{H} & 3.934881000 & 3.138584000 & 0.972365000 \\ \mathrm{H} & 4.076720000 & 3.095322000 & -0.786641000 \\ \mathrm{C} & 1.373502000 & -2.719386000 & -0.132184000 \\ \mathrm{C} & 0.967926000 & -3.279791000 & -1.378191000 \\ \mathrm{C} & 0.354529000 & -4.541946000 & -1.370836000 \\ \mathrm{H} & 0.040601000 & -4.991539000 & -2.318601000 \\ \mathrm{C} & 0.127459000 & -5.233157000 & -0.176901000 \\ \mathrm{H} & -0.358462000 & -6.214034000 & -0.194027000 \\ \mathrm{C} & 0.510812000 & -4.662064000 & 1.041060000 \\ \mathrm{H} & -0.313522000 & -5.204003000 & 1.971579000 \\ \mathrm{H} & 0.382799000 & -1.612499000 & 2.546663000 \\ \mathrm{C} & 1.132413000 & -3.405383000 & 1.094544000 \\ \mathrm{C} & 1.167453000 & -2.543329000 & -2.700624000 \\ \mathrm{H} & 1.691452000 & -1.599064000 & -2.478484000 \\ \mathrm{C} & 2.045921000 & -3.343107000 & -3.685733000 \\ \mathrm{H} & 3.029340000 & -3.589771000 & -3.252777000 \\ \mathrm{H} & 2.220234000 & -2.760979000 & -4.606401000 \\ \mathrm{H} & 1.567250000 & -4.291659000 & -3.982322000 \\ \mathrm{C} & -0.189985000 & -2.181336000 & -3.340539000 \\ \mathrm{H} & -0.809124000 & -1.580679000 & -2.656396000 \\ \mathrm{H} & -0.763401000 & -3.085643000 & -3.606397000 \\ \mathrm{H} & -0.038397000 & -1.600572000 & -4.266236000 \\ \mathrm{H} & 1.477553000 & -2.786391000 & 2.447279000 \\ \mathrm{H} & 2.094735000 & -1.891826000 & 2.260349000 \\ \mathrm{H} & 3.209238000 & -4.093372000 & 2.848915000 \\ \mathrm{H} & 1.712170000 & -4.621232000 & 3.649672000 \\ \mathrm{H} & 0.193389000 & -2.317178000 & 3.166804000 \\ \mathrm{H} & -14576000 & -1.817241000 & 4.118639000 \\ \mathrm{H} & -3.171440000 & 3.397869000 \\ \mathrm{H} & & & \\ \mathrm{H} & & & \end{array}$




\begin{tabular}{|c|c|c|c|}
\hline $\mathrm{C}$ & 1.375712000 & 2.718394000 & 0.132198000 \\
\hline $\mathrm{C}$ & 0.970574000 & 3.279106000 & 1.378209000 \\
\hline $\mathrm{C}$ & 0.358255000 & 4.541786000 & 1.370871000 \\
\hline $\mathrm{H}$ & 0.044696000 & 4.991631000 & 2.318639000 \\
\hline $\mathrm{C}$ & 0.131800000 & 5.233219000 & 0.176947000 \\
\hline $\mathrm{H}$ & -0.353280000 & 6.214511000 & 0.194086000 \\
\hline $\mathrm{C}$ & 0.514684000 & 4.661824000 & -1.041021000 \\
\hline $\mathrm{H}$ & 0.317869000 & 5.203951000 & -1.971531000 \\
\hline $\mathrm{C}$ & 1.135222000 & 3.404618000 & -1.094520000 \\
\hline $\mathrm{C}$ & 1.169459000 & 2.542448000 & 2.700630000 \\
\hline $\mathrm{H}$ & 1.692673000 & 1.597749000 & 2.478480000 \\
\hline $\mathrm{C}$ & 2.048582000 & 3.341468000 & 3.685768000 \\
\hline $\mathrm{H}$ & 3.032213000 & 3.587317000 & 3.252830000 \\
\hline $\mathrm{H}$ & 2.222395000 & 2.759175000 & 4.606427000 \\
\hline $\mathrm{H}$ & 1.570700000 & 4.290414000 & 3.982372000 \\
\hline $\mathrm{C}$ & -0.188289000 & 2.181574000 & 3.340519000 \\
\hline $\mathrm{H}$ & -0.760959000 & 3.086354000 & 3.606378000 \\
\hline $\mathrm{H}$ & -0.037196000 & 1.600677000 & 4.266214000 \\
\hline $\mathrm{H}$ & -0.807917000 & 1.581434000 & 2.656364000 \\
\hline $\mathrm{C}$ & 1.479866000 & 2.785361000 & -2.447257000 \\
\hline $\mathrm{H}$ & 2.096310000 & 1.890287000 & -2.260330000 \\
\hline $\mathrm{C}$ & 2.297627000 & 3.732637000 & -3.348774000 \\
\hline $\mathrm{H}$ & 3.212615000 & 4.090943000 & -2.848853000 \\
\hline $\mathrm{H}$ & 1.715991000 & 4.620013000 & -3.649642000 \\
\hline $\mathrm{H}$ & 2.599979000 & 3.213958000 & -4.273970000 \\
\hline $\mathrm{C}$ & 0.195320000 & 2.317215000 & -3.166796000 \\
\hline $\mathrm{H}$ & -0.462739000 & 3.172024000 & -3.397853000 \\
\hline $\mathrm{H}$ & -0.381450000 & 1.613004000 & -2.546666000 \\
\hline $\mathrm{H}$ & 0.443099000 & 1.817085000 & -4.118636000 \\
\hline $\mathrm{C}$ & -1.202997000 & 0.000491000 & -0.000014000 \\
\hline $\mathrm{C}$ & -2.446239000 & 0.000922000 & -0.000019000 \\
\hline $\mathrm{Cl}$ & -3.778702000 & -0.470788000 & 2.704872000 \\
\hline $\mathrm{Cl}$ & -3.778398000 & 0.473530000 & -2.704909000 \\
\hline $\mathrm{C}$ & -3.857615000 & 0.001399000 & -0.000018000 \\
\hline $\mathrm{C}$ & -4.618496000 & -0.205043000 & 1.189388000 \\
\hline $\mathrm{C}$ & -6.014100000 & -0.207080000 & 1.196960000 \\
\hline $\mathrm{H}$ & -6.547534000 & -0.371432000 & 2.135828000 \\
\hline $\mathrm{C}$ & -6.709930000 & 0.002361000 & -0.000010000 \\
\hline $\mathrm{H}$ & -7.803669000 & 0.002732000 & -0.000007000 \\
\hline $\mathrm{C}$ & -6.013965000 & 0.211330000 & -1.196985000 \\
\hline $\mathrm{H}$ & -6.547293000 & 0.376043000 & -2.135849000 \\
\hline $\mathrm{C}$ & -4.618363000 & 0.208350000 & -1.189421000 \\
\hline
\end{tabular}

Table S12. Geometry optimized structure of $\mathrm{T}$-shaped $\left[{ }^{i} \mathrm{Pr}_{2} \mathrm{NN}\right] \mathrm{Cu}-\mathrm{C} \equiv \mathrm{CAr}^{\mathrm{Cl} 2}$ (3) at BP86/6-311+G(d) level of theory.

$\begin{array}{llll}\mathrm{Cu} & 0.656423000 & 0.183982000 & -0.015337000\end{array}$ 


\begin{tabular}{|c|c|c|c|}
\hline $\mathrm{N}$ & 1.001707000 & 2.104313000 & 0.155846000 \\
\hline $\mathrm{N}$ & 2.505945000 & -0.305052000 & -0.140261000 \\
\hline $\mathrm{C}$ & 2.218322000 & 2.662327000 & 0.119944000 \\
\hline $\mathrm{C}$ & 3.416338000 & 1.923236000 & 0.017072000 \\
\hline $\mathrm{H}$ & 4.341638000 & 2.501604000 & 0.023645000 \\
\hline $\mathrm{C}$ & 3.554314000 & 0.525532000 & -0.091340000 \\
\hline $\mathrm{C}$ & 2.337121000 & 4.174269000 & 0.189969000 \\
\hline $\mathrm{H}$ & 3.384014000 & 4.482222000 & 0.317829000 \\
\hline $\mathrm{H}$ & 1.740595000 & 4.583011000 & 1.020051000 \\
\hline $\mathrm{H}$ & 1.951070000 & 4.640138000 & -0.731515000 \\
\hline $\mathrm{C}$ & 4.955638000 & -0.050152000 & -0.151178000 \\
\hline $\mathrm{H}$ & 5.707898000 & 0.746096000 & -0.235026000 \\
\hline $\mathrm{H}$ & 5.066867000 & -0.735638000 & -1.006224000 \\
\hline $\mathrm{H}$ & 5.177018000 & -0.640050000 & 0.753597000 \\
\hline $\mathrm{C}$ & -0.182593000 & 2.904428000 & 0.188028000 \\
\hline $\mathrm{C}$ & -0.825777000 & 3.139333000 & 1.436904000 \\
\hline $\mathrm{C}$ & -1.996136000 & 3.913785000 & 1.446078000 \\
\hline $\mathrm{H}$ & -2.500970000 & 4.111802000 & 2.397355000 \\
\hline $\mathrm{C}$ & -2.536009000 & 4.429771000 & 0.264175000 \\
\hline $\mathrm{H}$ & -3.454179000 & 5.025301000 & 0.293632000 \\
\hline $\mathrm{C}$ & -1.906643000 & 4.171789000 & -0.957953000 \\
\hline $\mathrm{H}$ & -2.346224000 & 4.566311000 & -1.879712000 \\
\hline $\mathrm{C}$ & -0.730332000 & 3.409975000 & -1.026969000 \\
\hline $\mathrm{C}$ & -0.283861000 & 2.573234000 & 2.747391000 \\
\hline $\mathrm{H}$ & 0.622219000 & 1.992642000 & 2.507474000 \\
\hline $\mathrm{C}$ & 0.124449000 & 3.688763000 & 3.733288000 \\
\hline $\mathrm{H}$ & 0.875431000 & 4.368436000 & 3.298070000 \\
\hline $\mathrm{H}$ & 0.556345000 & 3.254451000 & 4.650875000 \\
\hline $\mathrm{H}$ & -0.742074000 & 4.301605000 & 4.034355000 \\
\hline $\mathrm{C}$ & -1.295672000 & 1.606773000 & 3.398082000 \\
\hline $\mathrm{H}$ & -1.572735000 & 0.793575000 & 2.710055000 \\
\hline $\mathrm{H}$ & -2.221852000 & 2.128809000 & 3.692892000 \\
\hline $\mathrm{H}$ & -0.866910000 & 1.155734000 & 4.309140000 \\
\hline $\mathrm{C}$ & -0.108410000 & 3.098401000 & -2.386790000 \\
\hline $\mathrm{H}$ & 0.877614000 & 2.637771000 & -2.208844000 \\
\hline $\mathrm{C}$ & 0.115839000 & 4.359444000 & -3.246237000 \\
\hline $\mathrm{H}$ & 0.713682000 & 5.120138000 & -2.717566000 \\
\hline $\mathrm{H}$ & -0.836223000 & 4.832775000 & -3.539125000 \\
\hline $\mathrm{H}$ & 0.648162000 & 4.098955000 & -4.176453000 \\
\hline $\mathrm{C}$ & -0.967960000 & 2.064730000 & -3.147821000 \\
\hline $\mathrm{H}$ & -0.489907000 & 1.790323000 & -4.103837000 \\
\hline $\mathrm{H}$ & -1.966681000 & 2.473589000 & -3.376791000 \\
\hline $\mathrm{H}$ & -1.113733000 & 1.147138000 & -2.556821000 \\
\hline $\mathrm{C}$ & 2.660845000 & -1.726682000 & -0.169643000 \\
\hline $\mathrm{C}$ & 2.586738000 & -2.408022000 & -1.419819000 \\
\hline $\mathrm{C}$ & 2.707083000 & -3.806250000 & -1.422473000 \\
\hline
\end{tabular}




$\begin{array}{llll}\mathrm{H} & 2.657305000 & -4.347363000 & -2.373033000 \\ \mathrm{C} & 2.880533000 & -4.523373000 & -0.234932000 \\ \mathrm{H} & 2.966385000 & -5.614459000 & -0.259995000 \\ \mathrm{C} & 2.931296000 & -3.844524000 & 0.987185000 \\ \mathrm{H} & 3.050996000 & -4.417320000 & 1.912347000 \\ \mathrm{C} & 2.820775000 & -2.447445000 & 1.051196000 \\ \mathrm{C} & 2.366052000 & -1.664068000 & -2.734622000 \\ \mathrm{H} & 2.354968000 & -0.585217000 & -2.507433000 \\ \mathrm{C} & 3.505470000 & -1.915055000 & -3.744631000 \\ \mathrm{H} & 4.489940000 & -1.645180000 & -3.328272000 \\ \mathrm{H} & 3.349723000 & -1.315082000 & -4.657036000 \\ \mathrm{H} & 3.554427000 & -2.973256000 & -4.052094000 \\ \mathrm{C} & 0.995176000 & -2.023519000 & -3.347281000 \\ \mathrm{H} & 0.940397000 & -3.093734000 & -3.609768000 \\ \mathrm{H} & 0.820234000 & -1.445327000 & -4.270326000 \\ \mathrm{H} & 0.172680000 & -1.807238000 & -2.647621000 \\ \mathrm{C} & 2.811604000 & -1.746463000 & 2.407896000 \\ \mathrm{H} & 2.898269000 & -0.662098000 & 2.227293000 \\ \mathrm{C} & 3.993535000 & -2.163248000 & 3.306634000 \\ \mathrm{H} & 4.964410000 & -2.018712000 & 2.804679000 \\ \mathrm{H} & 3.930720000 & -3.222457000 & 3.607156000 \\ \mathrm{H} & 3.999679000 & -1.563682000 & 4.232232000 \\ \mathrm{C} & 1.465146000 & -1.985762000 & 3.127295000 \\ \mathrm{H} & 1.318914000 & -3.056834000 & 3.347439000 \\ \mathrm{H} & 0.612757000 & -1.654370000 & 2.512782000 \\ \mathrm{H} & 1.432754000 & -1.437971000 & 4.084454000 \\ \mathrm{C} & -1.074284000 & -0.532933000 & -0.022413000 \\ \mathrm{C} & -2.205129000 & -1.047253000 & -0.024151000 \\ \mathrm{Cl} & -3.684104000 & -1.048018000 & -2.694744000 \\ \mathrm{Cl} & -3.121423000 & -2.219785000 & 2.640807000 \\ \mathrm{C} & -3.477227000 & -1.660412000 & -0.025190000 \\ \mathrm{C} & -4.285173000 & -1.735975000 & -1.198456000 \\ \mathrm{C} & -5.544501000 & -2.337730000 & -1.206084000 \\ \mathrm{H} & -6.122289000 & -2.365844000 & -2.132600000 \\ \mathrm{H} & -7.033247000 & -3.372335000 & -0.025876000 \\ \mathrm{H} & -5.038706000 & -2.249019000 & 1.146832000\end{array}$

Table S13. Geometry optimized structure of $\left\{\left[{ }^{i} \mathrm{Pr}_{2} \mathrm{NN}\right] \mathrm{Cu}^{\mathrm{I}}\left(\mathrm{C} \equiv \mathrm{CAr}^{\mathrm{Cl}}\right)\right\}^{-}$(4) at $\mathrm{BP} 86 / 6$ $311+\mathrm{G}(\mathrm{d})$ level of theory.

$\begin{array}{lrrr}\mathrm{Cu} & 0.594456000 & 0.000062000 & 0.000005000 \\ \mathrm{~N} & 1.941613000 & -1.475669000 & 0.000011000 \\ \mathrm{~N} & 1.941221000 & 1.476146000 & 0.000011000 \\ \mathrm{C} & 3.266124000 & -1.279557000 & 0.000021000\end{array}$




\begin{tabular}{|c|c|c|c|}
\hline $\mathrm{C}$ & 3.872160000 & 0.000497000 & 0.000026000 \\
\hline $\mathrm{H}$ & 4.965230000 & 0.000643000 & 0.000035000 \\
\hline $\mathrm{C}$ & 3.265784000 & 1.280391000 & 0.000020000 \\
\hline $\mathrm{C}$ & 4.205059000 & -2.479028000 & 0.000028000 \\
\hline $\mathrm{H}$ & 5.258437000 & -2.161828000 & 0.000042000 \\
\hline $\mathrm{H}$ & 4.033690000 & -3.119559000 & -0.881235000 \\
\hline $\mathrm{H}$ & 4.033668000 & -3.119566000 & 0.881280000 \\
\hline $\mathrm{C}$ & 4.204398000 & 2.480113000 & 0.000024000 \\
\hline $\mathrm{H}$ & 5.257860000 & 2.163194000 & 0.000031000 \\
\hline $\mathrm{H}$ & 4.032841000 & 3.120601000 & 0.881280000 \\
\hline $\mathrm{H}$ & 4.032852000 & 3.120599000 & -0.881235000 \\
\hline $\mathrm{C}$ & 1.427429000 & -2.807594000 & 0.000005000 \\
\hline $\mathrm{C}$ & 1.134730000 & -3.457191000 & -1.233657000 \\
\hline $\mathrm{C}$ & 0.575065000 & -4.744922000 & -1.206826000 \\
\hline $\mathrm{H}$ & 0.338805000 & -5.246586000 & -2.152421000 \\
\hline $\mathrm{C}$ & 0.300183000 & -5.396661000 & -0.000008000 \\
\hline $\mathrm{H}$ & -0.139667000 & -6.400218000 & -0.000013000 \\
\hline $\mathrm{C}$ & 0.575034000 & -4.744921000 & 1.206817000 \\
\hline $\mathrm{H}$ & 0.338748000 & -5.246583000 & 2.152406000 \\
\hline $\mathrm{C}$ & 1.134698000 & -3.457190000 & 1.233661000 \\
\hline $\mathrm{C}$ & 1.357089000 & -2.751194000 & -2.569996000 \\
\hline $\mathrm{H}$ & 1.937042000 & -1.836658000 & -2.364225000 \\
\hline $\mathrm{C}$ & 2.164093000 & -3.600959000 & -3.572434000 \\
\hline $\mathrm{H}$ & 3.132163000 & -3.921078000 & -3.151226000 \\
\hline $\mathrm{H}$ & 2.369329000 & -3.022794000 & -4.490555000 \\
\hline $\mathrm{H}$ & 1.617698000 & -4.510976000 & -3.876279000 \\
\hline $\mathrm{C}$ & 0.007961000 & -2.310427000 & -3.177611000 \\
\hline $\mathrm{H}$ & -0.548034000 & -1.663634000 & -2.480856000 \\
\hline $\mathrm{H}$ & -0.628153000 & -3.183477000 & -3.407312000 \\
\hline $\mathrm{H}$ & 0.164521000 & -1.750409000 & -4.116833000 \\
\hline $\mathrm{C}$ & 1.357024000 & -2.751192000 & 2.570005000 \\
\hline $\mathrm{H}$ & 1.936972000 & -1.836650000 & 2.364247000 \\
\hline $\mathrm{C}$ & 2.164019000 & -3.600951000 & 3.572456000 \\
\hline $\mathrm{H}$ & 3.132101000 & -3.921057000 & 3.151266000 \\
\hline $\mathrm{H}$ & 1.617628000 & -4.510975000 & 3.876287000 \\
\hline $\mathrm{H}$ & 2.369230000 & -3.022787000 & 4.490583000 \\
\hline $\mathrm{C}$ & 0.007880000 & -2.310440000 & 3.177596000 \\
\hline $\mathrm{H}$ & 0.164418000 & -1.750422000 & 4.116822000 \\
\hline $\mathrm{H}$ & -0.628228000 & -3.183498000 & 3.407284000 \\
\hline $\mathrm{H}$ & -0.548109000 & -1.663653000 & 2.480832000 \\
\hline $\mathrm{C}$ & 1.426675000 & 2.807930000 & 0.000002000 \\
\hline $\mathrm{C}$ & 1.133778000 & 3.457455000 & 1.233656000 \\
\hline $\mathrm{C}$ & 0.573755000 & 4.745029000 & 1.206809000 \\
\hline $\mathrm{H}$ & 0.337344000 & 5.246634000 & 2.152398000 \\
\hline $\mathrm{C}$ & 0.298711000 & 5.396686000 & -0.000017000 \\
\hline $\mathrm{H}$ & -0.141419000 & 6.400121000 & -0.000024000 \\
\hline
\end{tabular}




$\begin{array}{lrrr}\mathrm{C} & 0.573765000 & 4.745017000 & -1.206834000 \\ \mathrm{H} & 0.337363000 & 5.246611000 & -2.152429000 \\ \mathrm{C} & 1.133789000 & 3.457442000 & -1.233662000 \\ \mathrm{C} & 1.356330000 & 2.751534000 & 2.570003000 \\ \mathrm{H} & 1.936503000 & 1.837137000 & 2.364235000 \\ \mathrm{C} & 2.163147000 & 3.601516000 & 3.572409000 \\ \mathrm{H} & 3.131124000 & 3.921875000 & 3.151171000 \\ \mathrm{H} & 2.368553000 & 3.023416000 & 4.490532000 \\ \mathrm{H} & 1.616528000 & 4.511398000 & 3.876254000 \\ \mathrm{C} & 0.007324000 & 2.310447000 & 3.177657000 \\ \mathrm{H} & -0.628999000 & 3.183345000 & 3.407358000 \\ \mathrm{H} & 0.164045000 & 1.750485000 & 4.116885000 \\ \mathrm{H} & -0.548530000 & 1.663506000 & 2.480928000 \\ \mathrm{C} & 1.356351000 & 2.751506000 & -2.569999000 \\ \mathrm{H} & 1.936530000 & 1.837116000 & -2.364219000 \\ \mathrm{C} & 2.163165000 & 3.601481000 & -3.572413000 \\ \mathrm{H} & 3.131138000 & 3.921854000 & -3.151176000 \\ \mathrm{H} & 1.616540000 & 4.511354000 & -3.876274000 \\ \mathrm{H} & 2.368580000 & 3.023370000 & -4.490528000 \\ \mathrm{C} & 0.007349000 & 2.310403000 & -3.177650000 \\ \mathrm{H} & -0.628977000 & 3.183294000 & -3.407366000 \\ \mathrm{H} & -0.548502000 & 1.663469000 & -2.480913000 \\ \mathrm{H} & 0.164076000 & 1.750429000 & -4.116871000 \\ \mathrm{C} & -1.259612000 & -0.000173000 & -0.000003000 \\ \mathrm{C} & -2.507904000 & -0.000316000 & -0.000007000 \\ \mathrm{Cl} & -3.858000000 & -0.000492000 & 2.751241000 \\ \mathrm{Cl} & -3.857988000 & -0.000467000 & -2.751263000 \\ \mathrm{C} & -3.912682000 & -0.000475000 & -0.000011000 \\ \mathrm{C} & -4.694275000 & -0.000568000 & 1.199926000 \\ \mathrm{C} & -6.089110000 & -0.000715000 & 1.211029000 \\ \mathrm{H} & -6.616142000 & -0.000776000 & 2.168839000 \\ \mathrm{C} & -6.795600000 & -0.000784000 & -0.000017000 \\ \mathrm{H} & -7.889793000 & -0.000898000 & -0.000020000 \\ \mathrm{C} & -6.089105000 & -0.000705000 & -1.211061000 \\ \mathrm{C} & -6.616132000 & -0.000757000 & -2.168873000 \\ & -4.694270000 & -0.000557000 & -1.199951000\end{array}$

Table S14. Geometry optimized structure of $\left\{\left[{ }^{i} \operatorname{Pr}_{2} \mathrm{NN}\right] \mathrm{Cu}{ }^{\mathrm{III}}\left(\mathrm{C} \equiv \mathrm{CAr}^{\mathrm{Cl} 2}\right)\right\}^{+}(9)$ at $\mathrm{BP} 86 / 6$ $311+\mathrm{G}(\mathrm{d})$ level of theory with charge $=+1$, multiplicity $=1$.
$\begin{array}{llll}\mathrm{Cu} & -0.700960000 & 0.017164000 & 0.004788000\end{array}$
$\mathrm{N} \quad-2.046049000 \quad-1.313677000 \quad 0.332118000$
$\begin{array}{llll}\mathrm{N} & -1.943380000 & 1.446894000 & -0.317600000\end{array}$
C $\quad-3.366600000 \quad-1.130175000 \quad 0.195312000$
$\begin{array}{llll}\mathrm{C} & -3.946257000 & 0.141528000 & 0.018013000\end{array}$
$\mathrm{H} \quad-5.036601000 \quad 0.183449000 \quad 0.023832000$
C $\quad-3.272569000 \quad 1.365039000 \quad-0.166905000$ 


\begin{tabular}{|c|c|c|c|}
\hline $\mathrm{C}$ & -4.295555000 & -2.324866000 & 0.256797000 \\
\hline $\mathrm{H}$ & -5.282499000 & -2.026512000 & 0.637666000 \\
\hline $\mathrm{H}$ & -3.886492000 & -3.122180000 & 0.893197000 \\
\hline $\mathrm{H}$ & -4.444998000 & -2.751399000 & -0.748822000 \\
\hline $\mathrm{C}$ & -4.108363000 & 2.627108000 & -0.220255000 \\
\hline $\mathrm{H}$ & -5.118340000 & 2.405130000 & -0.592843000 \\
\hline $\mathrm{H}$ & -3.645296000 & 3.391962000 & -0.859463000 \\
\hline $\mathrm{H}$ & -4.216319000 & 3.062244000 & 0.786948000 \\
\hline $\mathrm{C}$ & -1.426526000 & -2.585287000 & 0.269303000 \\
\hline $\mathrm{C}$ & -0.801618000 & -3.076565000 & 1.464795000 \\
\hline $\mathrm{C}$ & -0.218172000 & -4.346995000 & 1.416382000 \\
\hline $\mathrm{H}$ & 0.237758000 & -4.765561000 & 2.317490000 \\
\hline $\mathrm{C}$ & -0.202236000 & -5.094349000 & 0.231721000 \\
\hline $\mathrm{H}$ & 0.270597000 & -6.081037000 & 0.218055000 \\
\hline $\mathrm{C}$ & -0.772683000 & -4.581187000 & -0.940735000 \\
\hline $\mathrm{H}$ & -0.722480000 & -5.171352000 & -1.859896000 \\
\hline $\mathrm{C}$ & -1.390677000 & -3.326179000 & -0.959686000 \\
\hline $\mathrm{C}$ & -0.833166000 & -2.289966000 & 2.769607000 \\
\hline $\mathrm{H}$ & -1.062470000 & -1.239963000 & 2.518511000 \\
\hline $\mathrm{C}$ & -1.965653000 & -2.801308000 & 3.690626000 \\
\hline $\mathrm{H}$ & -2.954880000 & -2.728839000 & 3.210463000 \\
\hline $\mathrm{H}$ & -2.000103000 & -2.210721000 & 4.620613000 \\
\hline $\mathrm{H}$ & -1.805444000 & -3.856018000 & 3.969107000 \\
\hline $\mathrm{C}$ & 0.521846000 & -2.305635000 & 3.503546000 \\
\hline $\mathrm{H}$ & 1.339661000 & -1.960526000 & 2.852814000 \\
\hline $\mathrm{H}$ & 0.781220000 & -3.308252000 & 3.880547000 \\
\hline $\mathrm{H}$ & 0.480408000 & -1.636886000 & 4.378198000 \\
\hline $\mathrm{C}$ & -1.895350000 & -2.750417000 & -2.278982000 \\
\hline $\mathrm{H}$ & -2.519159000 & -1.867515000 & -2.059449000 \\
\hline $\mathrm{C}$ & -2.760345000 & -3.744030000 & -3.081013000 \\
\hline $\mathrm{H}$ & -3.591700000 & -4.151398000 & -2.483435000 \\
\hline $\mathrm{H}$ & -2.171314000 & -4.597998000 & -3.452169000 \\
\hline $\mathrm{H}$ & -3.190871000 & -3.244079000 & -3.963372000 \\
\hline $\mathrm{C}$ & -0.699173000 & -2.259001000 & -3.129210000 \\
\hline $\mathrm{H}$ & -1.057946000 & -1.784978000 & -4.057463000 \\
\hline $\mathrm{H}$ & -0.045001000 & -3.099854000 & -3.411844000 \\
\hline $\mathrm{H}$ & -0.080050000 & -1.528447000 & -2.583229000 \\
\hline $\mathrm{C}$ & -1.227830000 & 2.667525000 & -0.266274000 \\
\hline $\mathrm{C}$ & -0.576969000 & 3.102758000 & -1.469716000 \\
\hline $\mathrm{C}$ & 0.106429000 & 4.322501000 & -1.432454000 \\
\hline $\mathrm{H}$ & 0.587127000 & 4.699545000 & -2.338912000 \\
\hline $\mathrm{C}$ & 0.192396000 & 5.070935000 & -0.251259000 \\
\hline $\mathrm{H}$ & 0.742888000 & 6.016583000 & -0.246229000 \\
\hline $\mathrm{C}$ & -0.407050000 & 4.610105000 & 0.928273000 \\
\hline $\mathrm{H}$ & -0.301581000 & 5.198341000 & 1.844005000 \\
\hline $\mathrm{C}$ & -1.122831000 & 3.408261000 & 0.958649000 \\
\hline
\end{tabular}




$\begin{array}{lrrr}\mathrm{C} & -0.685017000 & 2.314291000 & -2.769229000 \\ \mathrm{H} & -0.964733000 & 1.279394000 & -2.505969000 \\ \mathrm{C} & -1.812535000 & 2.882976000 & -3.662518000 \\ \mathrm{H} & -2.791390000 & 2.864676000 & -3.156797000 \\ \mathrm{H} & -1.902725000 & 2.292841000 & -4.589062000 \\ \mathrm{H} & -1.603025000 & 3.926896000 & -3.948826000 \\ \mathrm{C} & 0.647194000 & 2.252088000 & -3.540887000 \\ \mathrm{H} & 0.946954000 & 3.235744000 & -3.937428000 \\ \mathrm{H} & 0.545773000 & 1.577647000 & -4.406175000 \\ \mathrm{H} & 1.464770000 & 1.871405000 & -2.909964000 \\ \mathrm{C} & -1.660325000 & 2.880449000 & 2.285064000 \\ \mathrm{H} & -2.351260000 & 2.046440000 & 2.075560000 \\ \mathrm{C} & -2.440958000 & 3.942410000 & 3.086067000 \\ \mathrm{H} & -3.242177000 & 4.409344000 & 2.490890000 \\ \mathrm{H} & -1.785294000 & 4.750483000 & 3.448109000 \\ \mathrm{H} & -2.902982000 & 3.482371000 & 3.974188000 \\ \mathrm{C} & -0.499404000 & 2.303869000 & 3.130643000 \\ \mathrm{H} & 0.219970000 & 3.093499000 & 3.402344000 \\ \mathrm{H} & 0.057071000 & 1.523683000 & 2.585988000 \\ \mathrm{H} & -0.886923000 & 1.865644000 & 4.064923000 \\ \mathrm{C} & 1.159802000 & -0.044048000 & 0.001083000 \\ \mathrm{C} & 2.406027000 & -0.079940000 & -0.001589000 \\ \mathrm{Cl} & 3.694900000 & -0.818266000 & -2.658713000 \\ \mathrm{Cl} & 3.748910000 & 0.584250000 & 2.648744000 \\ \mathrm{C} & 3.809322000 & -0.120133000 & -0.005091000 \\ \mathrm{C} & 4.552419000 & -0.449172000 & -1.185177000 \\ \mathrm{C} & 5.944974000 & -0.490890000 & -1.191069000 \\ \mathrm{H} & 6.475735000 & -0.748793000 & -2.109764000 \\ \mathrm{C} & 6.646708000 & -0.200124000 & -0.012742000 \\ \mathrm{H} & 7.739737000 & -0.230948000 & -0.015666000 \\ \mathrm{C} & 5.968824000 & 0.129687000 & 1.169257000 \\ \mathrm{H} & 6.518198000 & 0.357284000 & 2.085017000 \\ \mathrm{C} & 4.576128000 & 0.166513000 & 1.170871000\end{array}$

Table S15. Geometry optimized structure of $\left\{\left[{ }^{i} \mathrm{Pr}_{2} \mathrm{NN}\right] \mathrm{Cu}^{\mathrm{III}}\left(\mathrm{C} \equiv \mathrm{CAr}^{\mathrm{Cl}}\right)\right\}^{+}(9)$ at $\mathrm{BP} 86 / 6$ $311+\mathrm{G}(\mathrm{d})$ level of theory with charge $=+1$, multiplicity $=3$.

$\begin{array}{llrc}\mathrm{Cu} & 0.649438000 & -0.000053000 & 0.000124000 \\ \mathrm{~N} & 1.967328000 & -1.398510000 & -0.328689000 \\ \mathrm{~N} & 1.967480000 & 1.398283000 & 0.328873000 \\ \mathrm{C} & 3.291622000 & -1.256276000 & -0.265823000 \\ \mathrm{C} & 3.900777000 & -0.000232000 & 0.000165000 \\ \mathrm{H} & 4.993225000 & -0.000296000 & 0.000215000 \\ \mathrm{C} & 3.291755000 & 1.255897000 & 0.266078000 \\ \mathrm{C} & 4.211432000 & -2.438477000 & -0.481203000 \\ \mathrm{H} & 5.170034000 & -2.115215000 & -0.911322000 \\ \mathrm{H} & 3.755035000 & -3.192537000 & -1.137329000\end{array}$




\begin{tabular}{|c|c|c|c|}
\hline $\mathrm{H}$ & 4.432900000 & -2.933741000 & 0.479368000 \\
\hline $\mathrm{C}$ & 4.211738000 & 2.437970000 & 0.481447000 \\
\hline $\mathrm{H}$ & 5.169927000 & 2.114659000 & 0.912464000 \\
\hline $\mathrm{H}$ & 3.755104000 & 3.192567000 & 1.136779000 \\
\hline $\mathrm{H}$ & 4.434099000 & 2.932555000 & -0.479267000 \\
\hline $\mathrm{C}$ & 1.346499000 & -2.672489000 & -0.495519000 \\
\hline $\mathrm{C}$ & 0.721110000 & -2.958005000 & -1.748017000 \\
\hline $\mathrm{C}$ & 0.114956000 & -4.211815000 & -1.901833000 \\
\hline $\mathrm{H}$ & -0.346851000 & -4.471853000 & -2.858348000 \\
\hline $\mathrm{C}$ & 0.089205000 & -5.139765000 & -0.855019000 \\
\hline $\mathrm{H}$ & -0.395008000 & -6.110400000 & -0.997421000 \\
\hline $\mathrm{C}$ & 0.669780000 & -4.824416000 & 0.379222000 \\
\hline $\mathrm{H}$ & 0.614926000 & -5.550569000 & 1.195212000 \\
\hline $\mathrm{C}$ & 1.308164000 & -3.595916000 & 0.595151000 \\
\hline $\mathrm{C}$ & 0.767686000 & -1.976438000 & -2.915425000 \\
\hline $\mathrm{H}$ & 1.071166000 & -0.992644000 & -2.513002000 \\
\hline $\mathrm{C}$ & 1.837674000 & -2.396219000 & -3.949160000 \\
\hline $\mathrm{H}$ & 2.840886000 & -2.470094000 & -3.498780000 \\
\hline $\mathrm{H}$ & 1.890215000 & -1.663412000 & -4.770899000 \\
\hline $\mathrm{H}$ & 1.598709000 & -3.377740000 & -4.390782000 \\
\hline $\mathrm{C}$ & -0.607119000 & -1.792092000 & -3.586174000 \\
\hline $\mathrm{H}$ & -1.374018000 & -1.490623000 & -2.855991000 \\
\hline $\mathrm{H}$ & -0.953426000 & -2.712246000 & -4.083982000 \\
\hline $\mathrm{H}$ & -0.547848000 & -1.010625000 & -4.360822000 \\
\hline $\mathrm{C}$ & 1.832992000 & -3.246133000 & 1.986050000 \\
\hline $\mathrm{H}$ & 2.479510000 & -2.356183000 & 1.902273000 \\
\hline $\mathrm{C}$ & 2.676909000 & -4.372936000 & 2.615231000 \\
\hline $\mathrm{H}$ & 3.494163000 & -4.700292000 & 1.952488000 \\
\hline $\mathrm{H}$ & 2.069164000 & -5.260712000 & 2.852799000 \\
\hline $\mathrm{H}$ & 3.124606000 & -4.029066000 & 3.561524000 \\
\hline $\mathrm{C}$ & 0.656156000 & -2.860984000 & 2.913039000 \\
\hline $\mathrm{H}$ & 1.029081000 & -2.550947000 & 3.903079000 \\
\hline $\mathrm{H}$ & -0.022583000 & -3.716575000 & 3.062848000 \\
\hline $\mathrm{H}$ & 0.059769000 & -2.034429000 & 2.493490000 \\
\hline $\mathrm{C}$ & 1.346734000 & 2.672305000 & 0.495569000 \\
\hline $\mathrm{C}$ & 0.721082000 & 2.957840000 & 1.747945000 \\
\hline $\mathrm{C}$ & 0.115000000 & 4.211691000 & 1.901661000 \\
\hline $\mathrm{H}$ & -0.347020000 & 4.471740000 & 2.858068000 \\
\hline $\mathrm{C}$ & 0.089587000 & 5.139682000 & 0.854870000 \\
\hline $\mathrm{H}$ & -0.394569000 & 6.110357000 & 0.997196000 \\
\hline $\mathrm{C}$ & 0.670430000 & 4.824333000 & -0.379243000 \\
\hline $\mathrm{H}$ & 0.615839000 & 5.550525000 & -1.195215000 \\
\hline $\mathrm{C}$ & 1.308736000 & 3.595775000 & -0.595078000 \\
\hline $\mathrm{C}$ & 0.767458000 & 1.976231000 & 2.915320000 \\
\hline $\mathrm{H}$ & 1.070221000 & 0.992267000 & 2.512753000 \\
\hline $\mathrm{C}$ & 1.838096000 & 2.395373000 & 3.948652000 \\
\hline
\end{tabular}




$\begin{array}{lrrr}\mathrm{H} & 2.841184000 & 2.468646000 & 3.497899000 \\ \mathrm{H} & 1.890506000 & 1.662545000 & 4.770380000 \\ \mathrm{H} & 1.599868000 & 3.377045000 & 4.390335000 \\ \mathrm{C} & -0.607165000 & 1.792620000 & 3.586621000 \\ \mathrm{H} & -0.952699000 & 2.712877000 & 4.084775000 \\ \mathrm{H} & -0.548024000 & 1.010976000 & 4.361099000 \\ \mathrm{H} & -1.374573000 & 1.491734000 & 2.856731000 \\ \mathrm{C} & 1.833823000 & 3.245989000 & -1.985880000 \\ \mathrm{H} & 2.480219000 & 2.355961000 & -1.902010000 \\ \mathrm{C} & 2.678004000 & 4.372713000 & -2.614837000 \\ \mathrm{H} & 3.495137000 & 4.699960000 & -1.951893000 \\ \mathrm{H} & 2.070411000 & 5.260558000 & -2.852534000 \\ \mathrm{H} & 3.125883000 & 4.028810000 & -3.561031000 \\ \mathrm{C} & 0.657137000 & 2.861007000 & -2.913129000 \\ \mathrm{H} & -0.021469000 & 3.716681000 & -3.063071000 \\ \mathrm{H} & 0.060562000 & 2.034516000 & -2.493723000 \\ \mathrm{H} & 1.030239000 & 2.550939000 & -3.903093000 \\ \mathrm{C} & -1.209211000 & 0.000001000 & 0.000131000 \\ \mathrm{C} & -2.459544000 & 0.000100000 & 0.000058000 \\ \mathrm{Cl} & -3.768538000 & -2.008180000 & 1.867966000 \\ \mathrm{Cl} & -3.767756000 & 2.008643000 & -1.868099000 \\ \mathrm{C} & -3.860425000 & 0.000240000 & -0.000072000 \\ \mathrm{C} & -4.614425000 & -0.894849000 & 0.830449000 \\ \mathrm{C} & -6.006871000 & -0.894568000 & 0.831480000 \\ \mathrm{H} & -6.548880000 & -1.588341000 & 1.477306000 \\ \mathrm{C} & -6.695623000 & 0.000526000 & -0.000362000 \\ \mathrm{H} & -7.789102000 & 0.000636000 & -0.000473000 \\ \mathrm{C} & -6.006520000 & 0.895481000 & -0.832064000 \\ \mathrm{H} & -6.548257000 & 1.589363000 & -1.478002000 \\ \mathrm{C} & -4.614075000 & 0.895482000 & -0.830749000\end{array}$

Table S16. Geometry optimized structure of $\left\{\left[^{i} \mathrm{Pr}_{2} \mathrm{NN}\right] \mathrm{Cu}{ }^{\mathrm{II}}\left(\mathrm{C} \equiv \mathrm{CAr}^{\mathrm{Cl} 2}\right)_{2}\right\}^{-}$at $\mathrm{BP} 86 / 6-$ $311+\mathrm{G}(\mathrm{d})$ level of theory.

$\begin{array}{lrrr}\mathrm{Cu} & -0.043923000 & -0.843019000 & -0.208372000 \\ \mathrm{~N} & 1.177926000 & -1.873290000 & -1.511061000 \\ \mathrm{~N} & -1.117052000 & -2.525807000 & 0.267259000 \\ \mathrm{C} & -0.923350000 & -3.730929000 & -0.277408000 \\ \mathrm{C} & -0.013476000 & -3.984769000 & -1.330535000 \\ \mathrm{C} & 0.964491000 & -3.136310000 & -1.896872000 \\ \mathrm{H} & -0.032687000 & -5.003242000 & -1.726914000 \\ \mathrm{C} & 2.330665000 & -1.193727000 & -2.023451000 \\ \mathrm{C} & 2.167791000 & -0.218031000 & -3.045857000 \\ \mathrm{C} & 3.622549000 & -1.459822000 & -1.483274000 \\ \mathrm{C} & 3.306762000 & 0.441346000 & -3.537654000 \\ \mathrm{C} & 4.725483000 & -0.760468000 & -2.000861000 \\ \mathrm{H} & 3.190625000 & 1.188136000 & -4.330856000\end{array}$




\begin{tabular}{|c|c|c|c|}
\hline $\mathrm{H}$ & 5.721809000 & -0.948777000 & -1.584918000 \\
\hline $\mathrm{C}$ & 4.580252000 & 0.176671000 & -3.027664000 \\
\hline $\mathrm{H}$ & 5.454315000 & 0.710871000 & -3.416461000 \\
\hline $\mathrm{C}$ & 3.842562000 & -2.447446000 & -0.335204000 \\
\hline $\mathrm{H}$ & 2.890150000 & -2.972120000 & -0.155834000 \\
\hline $\mathrm{C}$ & 0.795782000 & 0.114662000 & -3.630031000 \\
\hline $\mathrm{H}$ & 0.041131000 & -0.401036000 & -3.014150000 \\
\hline $\mathrm{C}$ & -2.118472000 & -2.392291000 & 1.285807000 \\
\hline $\mathrm{C}$ & -1.757639000 & -2.563204000 & 2.650251000 \\
\hline $\mathrm{C}$ & -3.458406000 & -2.063482000 & 0.926727000 \\
\hline $\mathrm{C}$ & -2.753439000 & -2.435301000 & 3.634088000 \\
\hline $\mathrm{C}$ & -4.415025000 & -1.956401000 & 1.950144000 \\
\hline $\mathrm{H}$ & -2.483690000 & -2.561103000 & 4.688857000 \\
\hline $\mathrm{H}$ & -5.447877000 & -1.702397000 & 1.692097000 \\
\hline $\mathrm{C}$ & -4.076396000 & -2.145333000 & 3.294142000 \\
\hline $\mathrm{H}$ & -4.839891000 & -2.048412000 & 4.074148000 \\
\hline $\mathrm{C}$ & -3.863358000 & -1.827826000 & -0.530121000 \\
\hline $\mathrm{H}$ & -2.960750000 & -1.470369000 & -1.052780000 \\
\hline $\mathrm{C}$ & -0.320301000 & -2.859472000 & 3.074819000 \\
\hline $\mathrm{H}$ & 0.272851000 & -3.009533000 & 2.158125000 \\
\hline $\mathrm{C}$ & 2.660222000 & 2.382067000 & 1.589402000 \\
\hline $\mathrm{C}$ & 3.024458000 & 3.521040000 & 0.807412000 \\
\hline $\mathrm{C}$ & 3.157615000 & 2.401939000 & 2.926844000 \\
\hline $\mathrm{C}$ & 3.804764000 & 4.568445000 & 1.301879000 \\
\hline $\mathrm{C}$ & 3.936518000 & 3.440667000 & 3.440785000 \\
\hline $\mathrm{C}$ & 4.263292000 & 4.530189000 & 2.624325000 \\
\hline $\mathrm{C}$ & 1.199943000 & 0.399275000 & 0.599334000 \\
\hline $\mathrm{C}$ & 1.870649000 & 1.330716000 & 1.073436000 \\
\hline $\mathrm{Cl}$ & 2.777731000 & 1.056813000 & 3.996118000 \\
\hline $\mathrm{Cl}$ & 2.470891000 & 3.622537000 & -0.854715000 \\
\hline $\mathrm{H}$ & 4.281325000 & 3.389244000 & 4.476589000 \\
\hline $\mathrm{H}$ & 4.872970000 & 5.348855000 & 3.018683000 \\
\hline $\mathrm{H}$ & 4.044342000 & 5.410224000 & 0.647310000 \\
\hline $\mathrm{C}$ & -1.653077000 & -4.955104000 & 0.266417000 \\
\hline $\mathrm{H}$ & -1.099804000 & -5.359995000 & 1.132101000 \\
\hline $\mathrm{H}$ & -2.668257000 & -4.727226000 & 0.618198000 \\
\hline $\mathrm{H}$ & -1.705338000 & -5.751286000 & -0.491765000 \\
\hline $\mathrm{C}$ & -0.209558000 & -4.144092000 & 3.923393000 \\
\hline $\mathrm{H}$ & -0.724871000 & -4.038647000 & 4.894118000 \\
\hline $\mathrm{H}$ & -0.648903000 & -5.015879000 & 3.410385000 \\
\hline $\mathrm{H}$ & 0.848640000 & -4.373986000 & 4.138051000 \\
\hline $\mathrm{C}$ & 0.291934000 & -1.657624000 & 3.822207000 \\
\hline $\mathrm{H}$ & -0.271433000 & -1.429313000 & 4.744243000 \\
\hline $\mathrm{H}$ & 1.336321000 & -1.870802000 & 4.108559000 \\
\hline $\mathrm{H}$ & 0.297342000 & -0.756762000 & 3.190994000 \\
\hline $\mathrm{C}$ & -4.341017000 & -3.119473000 & -1.232304000 \\
\hline
\end{tabular}




$\begin{array}{lrrr}\mathrm{H} & -3.547708000 & -3.878550000 & -1.297405000 \\ \mathrm{H} & -5.199861000 & -3.567263000 & -0.700145000 \\ \mathrm{H} & -4.666515000 & -2.893921000 & -2.263218000 \\ \mathrm{C} & -4.938201000 & -0.735406000 & -0.680363000 \\ \mathrm{H} & -4.658287000 & 0.178394000 & -0.135994000 \\ \mathrm{H} & -5.055273000 & -0.468105000 & -1.743313000 \\ \mathrm{C} & 0.653087000 & -0.401595000 & -5.078582000 \\ \mathrm{H} & -0.355249000 & -0.181632000 & -5.470620000 \\ \mathrm{H} & 1.385938000 & 0.079250000 & -5.750960000 \\ \mathrm{H} & 0.807821000 & -1.491566000 & -5.143329000 \\ \mathrm{C} & 1.821650000 & -3.755828000 & -2.994782000 \\ \mathrm{H} & 1.988030000 & -3.048223000 & -3.821019000 \\ \mathrm{H} & 2.818936000 & -4.033736000 & -2.617297000 \\ \mathrm{H} & 1.346114000 & -4.664180000 & -3.394039000 \\ \mathrm{C} & 4.913334000 & -3.511463000 & -0.661824000 \\ \mathrm{H} & 5.916655000 & -3.061875000 & -0.760356000 \\ \mathrm{H} & 4.970100000 & -4.260675000 & 0.147103000 \\ \mathrm{H} & 4.700256000 & -4.046507000 & -1.602217000 \\ \mathrm{C} & 4.211409000 & -1.710378000 & 0.970035000 \\ \mathrm{H} & 5.167847000 & -1.168877000 & 0.862555000 \\ \mathrm{H} & 3.438394000 & -0.977933000 & 1.244108000 \\ \mathrm{H} & 4.324467000 & -2.427668000 & 1.802651000 \\ \mathrm{H} & -5.927250000 & -1.069145000 & -0.317540000 \\ \mathrm{C} & 0.487591000 & 1.622284000 & -3.554558000 \\ \mathrm{H} & 1.184632000 & 2.216431000 & -4.172241000 \\ \mathrm{H} & -0.534577000 & 1.822847000 & -3.915101000 \\ \mathrm{H} & 0.549954000 & 1.982153000 & -2.517386000 \\ \mathrm{C} & -1.404263000 & 0.519351000 & -0.375529000 \\ \mathrm{C} & -2.136741000 & 1.517693000 & -0.456437000 \\ \mathrm{C} & -2.977232000 & 2.649571000 & -0.521417000 \\ \mathrm{C} & -3.179857000 & 3.511515000 & 0.600455000 \\ \mathrm{C} & -3.689817000 & 3.029071000 & -1.697489000 \\ \mathrm{Cl} & -3.534615000 & 2.038139000 & -3.146327000 \\ \mathrm{C} 1 & -2.358821000 & 3.144318000 & 2.106690000 \\ \mathrm{H} & -4.004179000 & 4.637865000 & 0.554564000 \\ \mathrm{H} & -4.516269000 & 4.152633000 & -1.763818000 \\ \mathrm{H} & -4.67439631000 & 4.385912000 & -2.699427000 \\ \mathrm{H} & -113178000 & 5.256415000 & 1.448983000\end{array}$

Table S17. Geometry optimized structure of $\left[{ }^{i} \mathrm{Pr}_{2} \mathrm{NN}\right] \mathrm{Cu}{ }^{\mathrm{III}}\left(\mathrm{C} \equiv \mathrm{CAr}{ }^{\mathrm{Cl} 2}\right)_{2}$ constrained at $\mathrm{BP} 86 / 6-311+\mathrm{G}(\mathrm{d})$ level of theory with charge $=0$, multiplicity $=1$.
$\begin{array}{llll}\mathrm{Cu} & -0.000013000 & -0.887954000 & -0.000238000\end{array}$
$\mathrm{N} \quad 1.329233000 \quad-2.228205000 \quad-0.627404000$
$\mathrm{N} \quad-1.329472000 \quad-2.228357000 \quad 0.626503000$ 


\begin{tabular}{|c|c|c|c|}
\hline $\mathrm{C}$ & -1.132381000 & -3.554156000 & 0.571511000 \\
\hline $\mathrm{C}$ & -0.000314000 & -4.161039000 & -0.001111000 \\
\hline $\mathrm{C}$ & 1.131899000 & -3.554042000 & -0.573230000 \\
\hline $\mathrm{H}$ & -0.000460000 & -5.252616000 & -0.001464000 \\
\hline $\mathrm{C}$ & 2.618853000 & -1.760242000 & -1.070609000 \\
\hline $\mathrm{C}$ & 2.708794000 & -1.190497000 & -2.370325000 \\
\hline $\mathrm{C}$ & 3.763538000 & -1.876060000 & -0.231841000 \\
\hline $\mathrm{C}$ & 3.969560000 & -0.771218000 & -2.823673000 \\
\hline $\mathrm{C}$ & 4.992757000 & -1.414680000 & -0.731190000 \\
\hline $\mathrm{H}$ & 4.063977000 & -0.347253000 & -3.828308000 \\
\hline $\mathrm{H}$ & 5.885109000 & -1.481185000 & -0.100620000 \\
\hline $\mathrm{C}$ & 5.103633000 & -0.874102000 & -2.014685000 \\
\hline $\mathrm{H}$ & 6.074495000 & -0.526580000 & -2.382238000 \\
\hline $\mathrm{C}$ & 3.712484000 & -2.452277000 & 1.185453000 \\
\hline $\mathrm{H}$ & 2.715524000 & -2.897156000 & 1.341238000 \\
\hline $\mathrm{C}$ & 1.498038000 & -1.069516000 & -3.294489000 \\
\hline $\mathrm{H}$ & 0.597467000 & -1.293151000 & -2.698584000 \\
\hline $\mathrm{C}$ & -2.618874000 & -1.760345000 & 1.070270000 \\
\hline $\mathrm{C}$ & -2.708234000 & -1.190872000 & 2.370156000 \\
\hline $\mathrm{C}$ & -3.763889000 & -1.875859000 & 0.231933000 \\
\hline $\mathrm{C}$ & -3.968802000 & -0.771730000 & 2.824159000 \\
\hline $\mathrm{C}$ & -4.992896000 & -1.414581000 & 0.731927000 \\
\hline $\mathrm{H}$ & -4.062799000 & -0.348010000 & 3.828931000 \\
\hline $\mathrm{H}$ & -5.885503000 & -1.480889000 & 0.101696000 \\
\hline $\mathrm{C}$ & -5.103228000 & -0.874400000 & 2.015623000 \\
\hline $\mathrm{H}$ & -6.073932000 & -0.526992000 & 2.383700000 \\
\hline $\mathrm{C}$ & -3.713478000 & -2.451583000 & -1.185574000 \\
\hline $\mathrm{H}$ & -2.716613000 & -2.896447000 & -1.341987000 \\
\hline $\mathrm{C}$ & -1.497038000 & -1.070237000 & 3.293786000 \\
\hline $\mathrm{H}$ & -0.596614000 & -1.292004000 & 2.696947000 \\
\hline $\mathrm{C}$ & 2.864508000 & 2.522275000 & 0.951099000 \\
\hline $\mathrm{C}$ & 3.569828000 & 3.277038000 & -0.029894000 \\
\hline $\mathrm{C}$ & 3.060889000 & 2.931691000 & 2.301585000 \\
\hline $\mathrm{C}$ & 4.406661000 & 4.344158000 & 0.303019000 \\
\hline $\mathrm{C}$ & 3.895760000 & 3.994999000 & 2.650468000 \\
\hline $\mathrm{C}$ & 4.570219000 & 4.699651000 & 1.646679000 \\
\hline $\mathrm{C}$ & 1.346731000 & 0.436887000 & 0.350527000 \\
\hline $\mathrm{C}$ & 2.005225000 & 1.450667000 & 0.605406000 \\
\hline $\mathrm{Cl}$ & 2.224489000 & 2.081795000 & 3.587915000 \\
\hline $\mathrm{Cl}$ & 3.374982000 & 2.871258000 & -1.722106000 \\
\hline $\mathrm{H}$ & 4.010188000 & 4.265983000 & 3.702325000 \\
\hline $\mathrm{H}$ & 5.224169000 & 5.534759000 & 1.913459000 \\
\hline $\mathrm{H}$ & 4.920863000 & 4.890904000 & -0.490522000 \\
\hline $\mathrm{C}$ & -2.167018000 & -4.502001000 & 1.156174000 \\
\hline $\mathrm{H}$ & -2.627407000 & -4.089170000 & 2.064352000 \\
\hline $\mathrm{H}$ & -2.983401000 & -4.690379000 & 0.441962000 \\
\hline
\end{tabular}




\begin{tabular}{|c|c|c|c|}
\hline $\mathrm{H}$ & -1.700966000 & -5.468593000 & 1.395784000 \\
\hline $\mathrm{C}$ & -1.559463000 & -2.107114000 & 4.437360000 \\
\hline $\mathrm{H}$ & -2.444724000 & -1.945121000 & 5.075914000 \\
\hline $\mathrm{H}$ & -1.609759000 & -3.139946000 & 4.055462000 \\
\hline $\mathrm{H}$ & -0.665549000 & -2.030105000 & 5.079258000 \\
\hline $\mathrm{C}$ & -1.329222000 & 0.353860000 & 3.857290000 \\
\hline $\mathrm{H}$ & -2.169535000 & 0.640699000 & 4.512075000 \\
\hline $\mathrm{H}$ & -0.409304000 & 0.415343000 & 4.461501000 \\
\hline $\mathrm{H}$ & -1.253701000 & 1.097150000 & 3.050277000 \\
\hline $\mathrm{C}$ & -4.766120000 & -3.560637000 & -1.409725000 \\
\hline $\mathrm{H}$ & -4.729666000 & -4.346106000 & -0.637415000 \\
\hline $\mathrm{H}$ & -5.790579000 & -3.152699000 & -1.409689000 \\
\hline $\mathrm{H}$ & -4.609962000 & -4.042023000 & -2.389575000 \\
\hline $\mathrm{C}$ & -3.890883000 & -1.349324000 & -2.252447000 \\
\hline $\mathrm{H}$ & -3.119658000 & -0.572722000 & -2.161267000 \\
\hline $\mathrm{H}$ & -3.830952000 & -1.782198000 & -3.265827000 \\
\hline $\mathrm{C}$ & 1.562111000 & -2.104314000 & -4.439853000 \\
\hline $\mathrm{H}$ & 0.668408000 & -2.027107000 & -5.082020000 \\
\hline $\mathrm{H}$ & 2.447472000 & -1.940286000 & -5.077746000 \\
\hline $\mathrm{H}$ & 1.613315000 & -3.137775000 & -4.059775000 \\
\hline $\mathrm{C}$ & 2.166476000 & -4.501516000 & -1.158546000 \\
\hline $\mathrm{H}$ & 2.624739000 & -4.089104000 & -2.068023000 \\
\hline $\mathrm{H}$ & 2.984460000 & -4.688131000 & -0.445736000 \\
\hline $\mathrm{H}$ & 1.701085000 & -5.468862000 & -1.396351000 \\
\hline $\mathrm{C}$ & 4.764926000 & -3.561497000 & 1.409695000 \\
\hline $\mathrm{H}$ & 5.789451000 & -3.153729000 & 1.409921000 \\
\hline $\mathrm{H}$ & 4.608468000 & -4.042972000 & 2.389455000 \\
\hline $\mathrm{H}$ & 4.728513000 & -4.346907000 & 0.637319000 \\
\hline $\mathrm{C}$ & 3.889472000 & -1.350431000 & 2.252797000 \\
\hline $\mathrm{H}$ & 4.874516000 & -0.862746000 & 2.156982000 \\
\hline $\mathrm{H}$ & 3.118446000 & -0.573651000 & 2.161463000 \\
\hline $\mathrm{H}$ & 3.828925000 & -1.783642000 & 3.265996000 \\
\hline $\mathrm{H}$ & -4.875798000 & -0.861530000 & -2.155915000 \\
\hline $\mathrm{C}$ & 1.329304000 & 0.355419000 & -3.855635000 \\
\hline $\mathrm{H}$ & 2.169957000 & 0.644171000 & -4.509144000 \\
\hline $\mathrm{H}$ & 0.409857000 & 0.417095000 & -4.460549000 \\
\hline $\mathrm{H}$ & 1.252397000 & 1.097230000 & -3.047396000 \\
\hline $\mathrm{C}$ & -1.346798000 & 0.436961000 & -0.350590000 \\
\hline $\mathrm{C}$ & -2.005251000 & 1.450842000 & -0.605161000 \\
\hline $\mathrm{C}$ & -2.864492000 & 2.522568000 & -0.950572000 \\
\hline $\mathrm{C}$ & -3.570085000 & 3.276858000 & 0.030579000 \\
\hline $\mathrm{C}$ & -3.060542000 & 2.932587000 & -2.300927000 \\
\hline $\mathrm{Cl}$ & -2.223803000 & 2.083265000 & -3.587419000 \\
\hline $\mathrm{Cl}$ & -3.375690000 & 2.870289000 & 1.722661000 \\
\hline $\mathrm{C}$ & -4.406863000 & 4.344111000 & -0.302060000 \\
\hline $\mathrm{C}$ & -3.895341000 & 3.996036000 & -2.649539000 \\
\hline
\end{tabular}




$\begin{array}{llll}\mathrm{H} & -4.009506000 & 4.267500000 & -3.701300000 \\ \mathrm{C} & -4.570076000 & 4.700214000 & -1.645599000 \\ \mathrm{H} & -5.223978000 & 5.535427000 & -1.912169000 \\ \mathrm{H} & -4.921288000 & 4.890472000 & 0.491602000\end{array}$

Table S18. Geometry optimized structure of $\left[{ }^{i} \mathrm{Pr}_{2} \mathrm{NN}\right] \mathrm{Cu}{ }^{\mathrm{III}}\left(\mathrm{C} \equiv \mathrm{CAr}^{\mathrm{Cl}}\right)_{2}$ at $\mathrm{BP} 86 / 6-$ $311+\mathrm{G}(\mathrm{d})$ level of theory with charge $=0$, multiplicity $=3$.

$\begin{array}{lrrr}\mathrm{Cu} & -0.380792000 & -0.991469000 & 0.035912000 \\ \mathrm{~N} & -2.231730000 & -1.793453000 & 0.397561000 \\ \mathrm{~N} & 0.476992000 & -2.800907000 & -0.299571000 \\ \mathrm{C} & -0.177837000 & -3.959297000 & -0.356931000 \\ \mathrm{C} & -1.556267000 & -4.080258000 & -0.030438000 \\ \mathrm{C} & -2.513652000 & -3.095147000 & 0.335126000 \\ \mathrm{H} & -1.944989000 & -5.101558000 & -0.061099000 \\ \mathrm{C} & -3.297762000 & -0.874723000 & 0.691374000 \\ \mathrm{C} & -3.323571000 & -0.266858000 & 1.977653000 \\ \mathrm{C} & -4.275596000 & -0.561202000 & -0.292658000 \\ \mathrm{C} & -4.379449000 & 0.609718000 & 2.275569000 \\ \mathrm{C} & -5.296078000 & 0.339043000 & 0.056110000 \\ \mathrm{H} & -4.427445000 & 1.074573000 & 3.264542000 \\ \mathrm{H} & -6.052333000 & 0.604547000 & -0.689512000 \\ \mathrm{C} & -5.363964000 & 0.909544000 & 1.329669000 \\ \mathrm{H} & -6.175729000 & 1.600088000 & 1.580145000 \\ \mathrm{C} & -4.225012000 & -1.112031000 & -1.719351000 \\ \mathrm{H} & -3.456639000 & -1.902027000 & -1.756509000 \\ \mathrm{C} & -2.266992000 & -0.595152000 & 3.030581000 \\ \mathrm{H} & -1.350244000 & -0.892221000 & 2.489443000 \\ \mathrm{C} & 1.893148000 & -2.805195000 & -0.570280000 \\ \mathrm{C} & 2.348842000 & -2.687187000 & -1.909099000 \\ \mathrm{C} & 2.802137000 & -2.903053000 & 0.518006000 \\ \mathrm{C} & 3.735376000 & -2.699773000 & -2.137777000 \\ \mathrm{C} & 4.176551000 & -2.924423000 & 0.227417000 \\ \mathrm{H} & 4.109649000 & -2.604500000 & -3.162390000 \\ \mathrm{H} & 4.896031000 & -3.007425000 & 1.047749000 \\ \mathrm{C} & 4.645497000 & -2.829553000 & -1.085514000 \\ \mathrm{H} & 5.721374000 & -2.842916000 & -1.287232000 \\ \mathrm{C} & 2.318054000 & -3.029198000 & 1.963186000 \\ \mathrm{H} & 1.268890000 & -2.690012000 & 1.984968000 \\ \mathrm{C} & 1.387304000 & -2.506106000 & -3.083731000 \\ \mathrm{H} & 0.363226000 & -2.682619000 & -2.713612000 \\ \mathrm{C} & -0.918952000 & 3.279640000 & -1.413127000 \\ \mathrm{C} & -1.891260000 & 4.160796000 & -0.841927000 \\ \mathrm{C} & -0.029462000 & 3.869909000 & -2.365377000 \\ \mathrm{C} & -1.972335000 & 5.508818000 & -1.190848000 \\ \mathrm{C} & -0.104815000 & 5.215882000 & -2.722951000 \\ & -1.078047000 & 6.033357000 & -2.133213000\end{array}$




\begin{tabular}{|c|c|c|c|}
\hline $\mathrm{C}$ & -0.792180000 & 0.716571000 & -0.736068000 \\
\hline $\mathrm{C}$ & -0.837952000 & 1.924116000 & -1.051194000 \\
\hline $\mathrm{Cl}$ & 1.201984000 & 2.875424000 & -3.108561000 \\
\hline $\mathrm{Cl}$ & -3.018609000 & 3.527202000 & 0.333416000 \\
\hline $\mathrm{H}$ & 0.597516000 & 5.618296000 & -3.456298000 \\
\hline $\mathrm{H}$ & -1.139034000 & 7.089830000 & -2.409827000 \\
\hline $\mathrm{H}$ & -2.731718000 & 6.140688000 & -0.724851000 \\
\hline $\mathrm{C}$ & 0.512279000 & -5.238920000 & -0.798851000 \\
\hline $\mathrm{H}$ & 0.478437000 & -5.317358000 & -1.899434000 \\
\hline $\mathrm{H}$ & 1.571797000 & -5.262331000 & -0.512036000 \\
\hline $\mathrm{H}$ & 0.005996000 & -6.125354000 & -0.390024000 \\
\hline $\mathrm{C}$ & 1.647673000 & -3.509744000 & -4.226233000 \\
\hline $\mathrm{H}$ & 0.872868000 & -3.413692000 & -5.005424000 \\
\hline $\mathrm{H}$ & 2.620930000 & -3.332575000 & -4.713738000 \\
\hline $\mathrm{H}$ & 1.643589000 & -4.553194000 & -3.870177000 \\
\hline $\mathrm{C}$ & 1.434774000 & -1.055646000 & -3.610171000 \\
\hline $\mathrm{H}$ & 1.190669000 & -0.331152000 & -2.818547000 \\
\hline $\mathrm{H}$ & 2.437740000 & -0.809203000 & -3.998749000 \\
\hline $\mathrm{H}$ & 0.712465000 & -0.916463000 & -4.432547000 \\
\hline $\mathrm{C}$ & 2.349676000 & -4.496569000 & 2.448009000 \\
\hline $\mathrm{H}$ & 1.718678000 & -5.153975000 & 1.829183000 \\
\hline $\mathrm{H}$ & 3.376696000 & -4.899795000 & 2.422477000 \\
\hline $\mathrm{H}$ & 1.987304000 & -4.569086000 & 3.487733000 \\
\hline $\mathrm{C}$ & 3.105900000 & -2.131574000 & 2.936221000 \\
\hline $\mathrm{H}$ & 3.100193000 & -1.083610000 & 2.602016000 \\
\hline $\mathrm{H}$ & 2.651377000 & -2.172019000 & 3.940390000 \\
\hline $\mathrm{C}$ & -2.697088000 & -1.794713000 & 3.904289000 \\
\hline $\mathrm{H}$ & -2.878269000 & -2.700044000 & 3.303191000 \\
\hline $\mathrm{H}$ & -1.916063000 & -2.035884000 & 4.645319000 \\
\hline $\mathrm{H}$ & -3.625647000 & -1.566796000 & 4.455682000 \\
\hline $\mathrm{C}$ & -3.901584000 & -3.628768000 & 0.653328000 \\
\hline $\mathrm{H}$ & -4.451873000 & -2.966496000 & 1.334569000 \\
\hline $\mathrm{H}$ & -4.502907000 & -3.722747000 & -0.266197000 \\
\hline $\mathrm{H}$ & -3.833723000 & -4.631109000 & 1.102261000 \\
\hline $\mathrm{C}$ & -5.564806000 & -1.736690000 & -2.164545000 \\
\hline $\mathrm{H}$ & -6.353437000 & -0.973542000 & -2.273006000 \\
\hline $\mathrm{H}$ & -5.452129000 & -2.226232000 & -3.146520000 \\
\hline $\mathrm{H}$ & -5.935388000 & -2.489538000 & -1.449929000 \\
\hline $\mathrm{C}$ & -3.791982000 & -0.015535000 & -2.717062000 \\
\hline $\mathrm{H}$ & -4.521171000 & 0.812227000 & -2.735619000 \\
\hline $\mathrm{H}$ & -2.812052000 & 0.405231000 & -2.446674000 \\
\hline $\mathrm{H}$ & -3.724414000 & -0.427125000 & -3.738975000 \\
\hline $\mathrm{H}$ & 4.155283000 & -2.454302000 & 3.044473000 \\
\hline $\mathrm{C}$ & -1.897014000 & 0.609597000 & 3.913739000 \\
\hline $\mathrm{H}$ & -1.636445000 & 1.488521000 & 3.304816000 \\
\hline $\mathrm{H}$ & -2.714532000 & 0.892784000 & 4.598683000 \\
\hline
\end{tabular}




$\begin{array}{lrrr}\mathrm{H} & -1.024055000 & 0.361813000 & 4.539270000 \\ \mathrm{C} & 1.074887000 & 0.057838000 & 0.739319000 \\ \mathrm{C} & 1.918795000 & 0.942078000 & 0.989702000 \\ \mathrm{C} & 2.896559000 & 1.909771000 & 1.281890000 \\ \mathrm{C} & 4.068063000 & 2.069293000 & 0.476205000 \\ \mathrm{C} & 2.782247000 & 2.803781000 & 2.391575000 \\ \mathrm{Cl} & 1.385196000 & 2.685412000 & 3.439860000 \\ \mathrm{Cl} & 4.298300000 & 1.019687000 & -0.902011000 \\ \mathrm{C} & 5.037447000 & 3.034062000 & 0.751321000 \\ \mathrm{C} & 3.746442000 & 3.770360000 & 2.676660000 \\ \mathrm{H} & 3.611042000 & 4.428771000 & 3.537580000 \\ \mathrm{C} & 4.874101000 & 3.883764000 & 1.852997000 \\ \mathrm{H} & 5.632223000 & 4.641135000 & 2.072014000 \\ \mathrm{H} & 5.912997000 & 3.116442000 & 0.103461000\end{array}$

Table S19. Geometry optimized structure of $\left[{ }^{i} \mathrm{Pr}_{2} \mathrm{NN}\right] \mathrm{Cu}^{\mathrm{I}}\left({ }^{\mathrm{Cl} 2} \mathrm{ArC} \equiv \mathrm{C}-\mathrm{C} \equiv \mathrm{CAr}{ }^{\mathrm{Cl} 2}\right)$ at BP86/6-311+G(d) level of theory.

$\begin{array}{lrrr}\mathrm{Cu} & -0.752871000 & 0.576428000 & -0.168696000 \\ \mathrm{~N} & 0.209618000 & 2.240785000 & -0.520802000 \\ \mathrm{~N} & -2.578666000 & 1.291989000 & -0.127827000 \\ \mathrm{C} & -2.829073000 & 2.583056000 & -0.424502000 \\ \mathrm{C} & -1.837656000 & 3.530086000 & -0.757278000 \\ \mathrm{C} & -0.434353000 & 3.392922000 & -0.768760000 \\ \mathrm{H} & -2.209223000 & 4.529427000 & -0.990379000 \\ \mathrm{C} & 1.650660000 & 2.250428000 & -0.528992000 \\ \mathrm{C} & 2.354886000 & 2.516599000 & 0.676354000 \\ \mathrm{C} & 2.346894000 & 1.969031000 & -1.735588000 \\ \mathrm{C} & 3.758773000 & 2.458066000 & 0.656654000 \\ \mathrm{C} & 3.751282000 & 1.925519000 & -1.700595000 \\ \mathrm{H} & 4.317959000 & 2.654107000 & 1.577675000 \\ \mathrm{H} & 4.304345000 & 1.703809000 & -2.619390000 \\ \mathrm{C} & 4.457095000 & 2.161348000 & -0.517542000 \\ \mathrm{H} & 5.551228000 & 2.122975000 & -0.512281000 \\ \mathrm{C} & 1.613809000 & 1.732089000 & -3.057455000 \\ \mathrm{H} & 0.540026000 & 1.903422000 & -2.876552000 \\ \mathrm{C} & 1.624231000 & 2.868311000 & 1.973347000 \\ \mathrm{H} & 0.563152000 & 3.029680000 & 1.722098000 \\ \mathrm{C} & -3.715114000 & 0.507517000 & 0.280773000 \\ \mathrm{C} & -4.482489000 & -0.181753000 & -0.700030000 \\ \mathrm{C} & -4.071572000 & 0.468332000 & 1.659672000 \\ \mathrm{C} & -5.674564000 & -0.804220000 & -0.287800000 \\ \mathrm{C} & -5.274664000 & -0.169731000 & 2.010580000 \\ \mathrm{H} & -6.292780000 & -1.326371000 & -1.023404000 \\ \mathrm{H} & -5.581859000 & -0.202097000 & 3.059601000 \\ \mathrm{C} & -6.085798000 & -0.775791000 & 1.047259000 \\ \mathrm{H} & -7.025058000 & -1.254803000 & 1.343028000\end{array}$




\begin{tabular}{|c|c|c|c|}
\hline $\mathrm{C}$ & -3.163803000 & 1.056902000 & 2.750103000 \\
\hline $\mathrm{H}$ & -2.124134000 & 0.864547000 & 2.423780000 \\
\hline $\mathrm{C}$ & -4.024457000 & -0.279691000 & -2.161404000 \\
\hline $\mathrm{H}$ & -2.920273000 & -0.332837000 & -2.139612000 \\
\hline $\mathrm{C}$ & 4.488002000 & -1.540388000 & 0.476070000 \\
\hline $\mathrm{C}$ & 5.097641000 & -1.556745000 & 1.763021000 \\
\hline $\mathrm{C}$ & 5.338639000 & -1.845801000 & -0.624337000 \\
\hline $\mathrm{C}$ & 6.456231000 & -1.824188000 & 1.939281000 \\
\hline $\mathrm{C}$ & 6.698571000 & -2.114812000 & -0.460916000 \\
\hline $\mathrm{C}$ & 7.255487000 & -2.097653000 & 0.823157000 \\
\hline $\mathrm{C}$ & 1.906995000 & -1.073361000 & 0.166850000 \\
\hline $\mathrm{C}$ & 3.114184000 & -1.262401000 & 0.305096000 \\
\hline $\mathrm{Cl}$ & 4.659208000 & -1.911843000 & -2.235743000 \\
\hline $\mathrm{Cl}$ & 4.113431000 & -1.253366000 & 3.178724000 \\
\hline $\mathrm{H}$ & 7.309956000 & -2.342593000 & -1.336791000 \\
\hline $\mathrm{H}$ & 8.320399000 & -2.308052000 & 0.956177000 \\
\hline $\mathrm{H}$ & 6.877442000 & -1.823457000 & 2.946952000 \\
\hline $\mathrm{C}$ & -4.256003000 & 3.116459000 & -0.373328000 \\
\hline $\mathrm{H}$ & -4.310953000 & 4.111625000 & -0.836647000 \\
\hline $\mathrm{H}$ & -4.971097000 & 2.453439000 & -0.877888000 \\
\hline $\mathrm{H}$ & -4.605996000 & 3.207320000 & 0.666806000 \\
\hline $\mathrm{C}$ & -4.394984000 & 0.942171000 & -3.034198000 \\
\hline $\mathrm{H}$ & -4.120490000 & 0.747108000 & -4.085054000 \\
\hline $\mathrm{H}$ & -5.481502000 & 1.135694000 & -3.007187000 \\
\hline $\mathrm{H}$ & -3.871478000 & 1.857537000 & -2.726367000 \\
\hline $\mathrm{C}$ & -4.542327000 & -1.552562000 & -2.859821000 \\
\hline $\mathrm{H}$ & -4.387803000 & -2.455038000 & -2.248814000 \\
\hline $\mathrm{H}$ & -5.618521000 & -1.482343000 & -3.094813000 \\
\hline $\mathrm{H}$ & -4.014365000 & -1.695458000 & -3.816328000 \\
\hline $\mathrm{C}$ & -3.300685000 & 2.584858000 & 2.944843000 \\
\hline $\mathrm{H}$ & -2.947003000 & 3.157124000 & 2.076493000 \\
\hline $\mathrm{H}$ & -4.348866000 & 2.865283000 & 3.148041000 \\
\hline $\mathrm{H}$ & -2.700629000 & 2.906749000 & 3.813200000 \\
\hline $\mathrm{C}$ & -3.355899000 & 0.376431000 & 4.120640000 \\
\hline $\mathrm{H}$ & -3.362349000 & -0.720916000 & 4.047248000 \\
\hline $\mathrm{H}$ & -2.537137000 & 0.667266000 & 4.799304000 \\
\hline $\mathrm{C}$ & 2.152959000 & 4.170583000 & 2.610496000 \\
\hline $\mathrm{H}$ & 2.132801000 & 5.014028000 & 1.901025000 \\
\hline $\mathrm{H}$ & 1.537726000 & 4.449089000 & 3.482792000 \\
\hline $\mathrm{H}$ & 3.191769000 & 4.062728000 & 2.965352000 \\
\hline $\mathrm{C}$ & 0.367403000 & 4.648963000 & -1.062622000 \\
\hline $\mathrm{H}$ & 1.065157000 & 4.497097000 & -1.900322000 \\
\hline $\mathrm{H}$ & -0.294932000 & 5.490927000 & -1.306013000 \\
\hline $\mathrm{H}$ & 0.985342000 & 4.934574000 & -0.195839000 \\
\hline $\mathrm{C}$ & 2.064995000 & 2.718384000 & -4.156496000 \\
\hline $\mathrm{H}$ & 1.461261000 & 2.580264000 & -5.069519000 \\
\hline
\end{tabular}




$\begin{array}{lrrr}\mathrm{H} & 1.958693000 & 3.768723000 & -3.839999000 \\ \mathrm{H} & 3.121175000 & 2.563369000 & -4.434622000 \\ \mathrm{C} & 1.771806000 & 0.279394000 & -3.551293000 \\ \mathrm{H} & 1.420285000 & -0.445795000 & -2.801740000 \\ \mathrm{H} & 1.193176000 & 0.121126000 & -4.477643000 \\ \mathrm{H} & 2.826554000 & 0.045706000 & -3.771829000 \\ \mathrm{H} & -4.296747000 & 0.690424000 & 4.605091000 \\ \mathrm{C} & 1.683089000 & 1.710585000 & 2.991005000 \\ \mathrm{H} & 1.264695000 & 0.781559000 & 2.573178000 \\ \mathrm{H} & 2.722079000 & 1.497685000 & 3.291723000 \\ \mathrm{H} & 1.113573000 & 1.963490000 & 3.901955000 \\ \mathrm{C} & 0.554006000 & -0.931248000 & 0.039376000 \\ \mathrm{C} & -0.637659000 & -1.394311000 & -0.012447000 \\ \mathrm{C} & -1.483574000 & -2.560501000 & -0.048453000 \\ \mathrm{C} & -1.758850000 & -3.241153000 & -1.262035000 \\ \mathrm{C} & -2.017674000 & -3.138219000 & 1.130423000 \\ \mathrm{Cl} & -1.605751000 & -2.434447000 & 2.681275000 \\ \mathrm{Cl} & -1.057844000 & -2.647824000 & -2.757903000 \\ \mathrm{C} & -2.541681000 & -4.397526000 & -1.305566000 \\ \mathrm{C} & -2.810573000 & -4.287471000 & 1.105370000 \\ \mathrm{H} & -3.198819000 & -4.693956000 & 2.041671000 \\ \mathrm{C} & -3.077273000 & -4.910292000 & -0.118743000 \\ \mathrm{H} & -3.695849000 & -5.811682000 & -0.146769000 \\ \mathrm{H} & -2.719572000 & -4.890460000 & -2.263798000\end{array}$

Table S20. Geometry optimized structure of $\left[{ }^{i} \mathrm{Pr}_{2} \mathrm{NN}\right] \mathrm{Cu}^{\mathrm{II}}\left(\mathrm{C} \equiv \mathrm{CAr}^{\mathrm{Cl}}\right)(\mathrm{NCMe})$ at $\mathrm{BP} 86 / 6$ $311+\mathrm{G}(\mathrm{d})$ level of theory.

$\begin{array}{lrrr}\mathrm{Cu} & 0.629535000 & -0.276946000 & 0.028960000 \\ \mathrm{~N} & 0.265040000 & -2.251634000 & -0.070186000 \\ \mathrm{~N} & 2.480745000 & -0.376625000 & -0.618910000 \\ \mathrm{C} & 3.070255000 & -1.490936000 & -1.058114000 \\ \mathrm{C} & 2.457922000 & -2.763376000 & -0.996948000 \\ \mathrm{C} & 1.167085000 & -3.119618000 & -0.551458000 \\ \mathrm{H} & 3.068899000 & -3.589638000 & -1.369900000 \\ \mathrm{C} & -1.086905000 & -2.679187000 & 0.113389000 \\ \mathrm{C} & -1.578611000 & -2.825718000 & 1.441369000 \\ \mathrm{C} & -1.930478000 & -2.927717000 & -1.009868000 \\ \mathrm{C} & -2.891436000 & -3.288321000 & 1.621516000 \\ \mathrm{C} & -3.240172000 & -3.371690000 & -0.769126000 \\ \mathrm{H} & -3.277268000 & -3.429309000 & 2.636351000 \\ \mathrm{H} & -3.904718000 & -3.565177000 & -1.617290000 \\ \mathrm{C} & -3.718848000 & -3.566914000 & 0.530233000 \\ \mathrm{H} & -4.741565000 & -3.923043000 & 0.691569000 \\ \mathrm{C} & -1.482566000 & -2.665214000 & -2.448505000 \\ \mathrm{H} & -0.389598000 & -2.521854000 & -2.446334000 \\ \mathrm{C} & -0.703086000 & -2.530642000 & 2.656698000\end{array}$




\begin{tabular}{|c|c|c|c|}
\hline & 0.194744000 & -2.004363000 & 0 \\
\hline & 3.183819000 & 0.862541000 & -0.497784000 \\
\hline & 2.813632000 & 1.934376000 & -1.367568000 \\
\hline & 4.178937000 & 1.051494000 & 0.510096000 \\
\hline & 3.436163000 & 3.180363000 & \\
\hline & 4.758289000 & 2.324847000 & 0.6 \\
\hline & 3.169497000 & 4.00837 & -1.8 \\
\hline & 5.518847000 & 2.490246000 & 1.408058000 \\
\hline & 4.393030000 & 3.384409000 & -0.19 \\
\hline & 4.861379000 & 4.366744000 & -0.07 \\
\hline & 4.655 & -0.06 & \\
\hline & 735000 & -0.98 & 1. \\
\hline & 1.842438000 & 1.722036000 & -2.52 \\
\hline & 1.2 & 0.8 & -2.3 \\
\hline & 7000 & 2.2 & 0.0 \\
\hline & -4.5 & & -0.0 \\
\hline & -3.12 & 3.6 & 0.1 \\
\hline & -5.6 & 2.4 & -0.0 \\
\hline & 56000 & 4.4 & 0.1 \\
\hline & -5.5 & $3.8^{\prime}$ & \\
\hline r & 1.0 & 0.6 & 2.0 \\
\hline & 0.6 & 1.5 & \\
\hline & 0.0 & 2.6 & \\
\hline & -0.6 & 3.2 & 2.7 \\
\hline $\mathrm{H}$ & -0. & 2.3 & 4.2 \\
\hline & 0.8 & 3.3 & 3.7 \\
\hline & -1.0 & 0.6 & -0.0 \\
\hline & -2.02 & 1.4 & -0.0 \\
\hline C & -1.5 & 4.4 & 0.2 \\
\hline & -4.69 & -0.0 & -0.1 \\
\hline $\mathrm{H}$ & & & \\
\hline H & -6.41 & & 0.0 \\
\hline $\mathrm{H}$ & -6.630 & 000 & -0.1 \\
\hline & 4.4 & -1.4 & -1.6 \\
\hline & 4.41 & -1.9 & -2.6 \\
\hline $\mathrm{H}$ & 4.8 & -0.4 & -1.7 \\
\hline $\mathrm{H}$ & 5.173957000 & -2.03 & -1.08428000 \\
\hline$C$ & 2.62 & 1.4 & -3.8 \\
\hline $\mathrm{H}$ & 1.926947000 & 6000 & -4.6 \\
\hline $\mathrm{H}$ & 3.24 & 2.30 & -4.11561000 \\
\hline $\mathrm{H}$ & 3.287101000 & 78000 & -3.73161900 \\
\hline - & 0.85 & 2.88 & -2.724 \\
\hline $\mathrm{H}$ & 0.287 & 3.089629000 & -1.80462700 \\
\hline & 1.358459000 & 3.815957000 & -3.03829900 \\
\hline 11 & 0.128229000 & 2.631404000 & -3.51379900 \\
\hline$C$ & 4.366521000 & 0.243577000 & 2.92773200 \\
\hline
\end{tabular}




$\begin{array}{rrrr}\mathrm{H} & 4.894367000 & 1.153736000 & 3.260574000 \\ \mathrm{H} & 3.291603000 & 0.385960000 & 3.102638000 \\ \mathrm{H} & 4.711824000 & -0.588648000 & 3.564832000 \\ \mathrm{C} & 6.165432000 & -0.345387000 & 1.259347000 \\ \mathrm{H} & 6.431825000 & -0.534448000 & 0.208350000 \\ \mathrm{H} & 6.775146000 & 0.506986000 & 1.603728000 \\ \mathrm{C} & -0.233661000 & -3.829969000 & 3.346673000 \\ \mathrm{H} & 0.334128000 & -4.475288000 & 2.656820000 \\ \mathrm{H} & 0.420067000 & -3.601398000 & 4.205973000 \\ \mathrm{H} & -1.089662000 & -4.416307000 & 3.722986000 \\ \mathrm{C} & 0.814769000 & -4.597300000 & -0.645419000 \\ \mathrm{H} & 0.179336000 & -4.914154000 & 0.194161000 \\ \mathrm{H} & 0.254506000 & -4.815656000 & -1.568779000 \\ \mathrm{H} & 1.727686000 & -5.210351000 & -0.655750000 \\ \mathrm{C} & -1.797926000 & -3.837188000 & -3.401732000 \\ \mathrm{H} & -1.351575000 & -3.652802000 & -4.393334000 \\ \mathrm{H} & -1.407861000 & -4.798868000 & -3.029329000 \\ \mathrm{H} & -2.883110000 & -3.960480000 & -3.555022000 \\ \mathrm{C} & -2.109316000 & -1.358059000 & -2.983209000 \\ \mathrm{H} & -1.867091000 & -0.501898000 & -2.336086000 \\ \mathrm{H} & -1.742335000 & -1.144246000 & -4.002223000 \\ \mathrm{H} & -3.208544000 & -1.438424000 & -3.030436000 \\ \mathrm{H} & 6.468672000 & -1.225195000 & 1.851945000 \\ \mathrm{C} & -1.408281000 & -1.599687000 & 3.662937000 \\ \mathrm{H} & -1.760239000 & -0.680175000 & 3.170083000 \\ \mathrm{H} & -2.279377000 & -2.083116000 & 4.136946000 \\ \mathrm{H} & -0.713000000 & -1.316831000 & 4.471740000\end{array}$

Table S21. Geometry optimized structure of $\left\{\left[{ }^{i} \mathrm{Pr}_{2} \mathrm{NN}\right] \mathrm{Cu}^{\mathrm{III}}\left(\mathrm{C} \equiv \mathrm{CAr}^{\mathrm{Cl} 2}\right)(\mathrm{NCMe})\right\}^{+}$at BP86/6-311+G(d) level of theory (multiplicity $=1$ ).

$\begin{array}{lccc}\mathrm{Cu} & -0.638546000 & 0.142974000 & -0.077277000 \\ \mathrm{~N} & -0.525171000 & 2.107628000 & -0.386402000 \\ \mathrm{~N} & -2.402477000 & -0.122409000 & -0.865792000 \\ \mathrm{C} & -3.052834000 & 0.811827000 & -1.568502000 \\ \mathrm{C} & -2.565010000 & 2.126852000 & -1.718124000 \\ \mathrm{C} & -1.411289000 & 2.735126000 & -1.175587000 \\ \mathrm{H} & -3.190424000 & 2.785916000 & -2.325793000 \\ \mathrm{C} & 0.723062000 & 2.710928000 & -0.074729000 \\ \mathrm{C} & 0.982296000 & 2.983649000 & 1.307891000 \\ \mathrm{C} & 1.691344000 & 3.000623000 & -1.090064000 \\ \mathrm{C} & 2.202892000 & 3.581021000 & 1.641007000 \\ \mathrm{C} & 2.903363000 & 3.572786000 & -0.682996000 \\ \mathrm{H} & 2.416609000 & 3.828258000 & 2.684047000 \\ \mathrm{H} & 3.669751000 & 3.792446000 & -1.431644000 \\ \mathrm{C} & 3.161546000 & 3.865150000 & 0.661196000 \\ \mathrm{H} & 4.117026000 & 4.315367000 & 0.947038000\end{array}$




\begin{tabular}{|c|c|c|c|}
\hline $\mathrm{C}$ & 1.503732000 & 00 & \\
\hline $\mathrm{H}$ & 0.460512000 & 2.331634000 & \\
\hline & .071845000 & .735799000 & 政 \\
\hline & 7000 & 89000 & \\
\hline & -3.032253000 & 1.321386000 & \\
\hline & 228000 & 2.561643000 & \\
\hline & -4.138577000 & -1.293050000 & \\
\hline 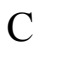 & 29000 & 086000 & \\
\hline $\mathrm{C}$ & -4.670936000 & -2.52 & \\
\hline $\mathrm{H}$ & 1000 & -4.7 & \\
\hline 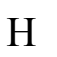 & -5.50 & -2.5 & \\
\hline & 26000 & 5000 & \\
\hline $\mathrm{H}$ & -4.6 & -4.6 & \\
\hline & 2000 & -0.0 & 1.0 \\
\hline $\mathrm{H}$ & -4.2 & & \\
\hline $\mathrm{C}$ & $-1.3 \xi$ & -2.6 & \\
\hline $\mathrm{H}$ & -0.8 & -1.6 & \\
\hline O & 3.5 & -1.6 & \\
\hline$y$ & 4.12 & -1.7 & \\
\hline $\mathrm{C}$ & 4.2 & -2.2 & \\
\hline & $5.3^{\prime}$ & -2.4 & \\
\hline $\mathrm{C}$ & 5.4 & -2.8 & \\
\hline & 6.0 & -2.9 & \\
\hline iv & -1.0 & -0.7 & \\
\hline $\mathrm{C}$ & -0.7 & -1.4 & 2. \\
\hline $\mathrm{C}$ & -0.3 & -2.28 & \\
\hline $\mathrm{H}$ & 0.7 & -2.4 & \\
\hline 11 & -0.5 & -1.8 & \\
\hline $\mathrm{H}$ & -0.8 & -3.2 & 3. \\
\hline & 1.12 & -0.5 & -0. \\
\hline $\mathrm{C}$ & 2.27 & -1.0 & \\
\hline $\mathrm{Cl}$ & 3.51 & -2.1 & -2 \\
\hline $\mathrm{Cl}$ & 3.36 & -1.0 & \\
\hline $\mathrm{H}$ & 5.94 & -3.3 & -1. \\
\hline $\mathrm{H}$ & 6.9 & -3.4 & \\
\hline $\mathrm{H}$ & 5.8 & -2.4 & 2.6 \\
\hline $\mathrm{C}$ & -4.3 & 0.4 & -2.2 \\
\hline $\mathrm{H}$ & -4.2 & 0.8 & -3 \\
\hline $\mathrm{H}$ & $-4.5 \mathrm{c}$ & -0.5 & -2. \\
\hline $\mathrm{H}$ & 00000 & 1.0 & -1.8 \\
\hline $\mathrm{C}$ & 510000 & 70000 & -3.2 \\
\hline $\mathrm{H}$ & & -2.7 & -4 \\
\hline $\mathrm{H}$ & 211000 & 938000 & -3.4672290 \\
\hline $\mathrm{H}$ & -2.69 & -1.95 & -3.6249 \\
\hline $\mathrm{C}$ & -0.345058000 & -3.721133000 & -1.69606100 \\
\hline $\mathrm{H}$ & 0.078681000 & -3.635652000 & -0.68374400 \\
\hline
\end{tabular}




$\begin{array}{lrrr}\mathrm{H} & -0.767601000 & -4.732797000 & -1.811778000 \\ \mathrm{H} & 0.487943000 & -3.635331000 & -2.410673000 \\ \mathrm{C} & -6.250659000 & 0.089007000 & 0.812955000 \\ \mathrm{H} & -6.520249000 & -0.059528000 & -0.244085000 \\ \mathrm{H} & -6.802055000 & -0.663337000 & 1.399772000 \\ \mathrm{H} & -6.623401000 & 1.078103000 & 1.124873000 \\ \mathrm{C} & -4.422772000 & 0.124081000 & 2.563231000 \\ \mathrm{H} & -3.340801000 & 0.122520000 & 2.758927000 \\ \mathrm{H} & -4.842460000 & 1.064949000 & 2.955301000 \\ \mathrm{C} & -0.926015000 & 4.005948000 & 2.605350000 \\ \mathrm{H} & -1.426524000 & 4.334433000 & 1.680326000 \\ \mathrm{H} & -1.706021000 & 3.819097000 & 3.361837000 \\ \mathrm{H} & -0.303701000 & 4.842641000 & 2.963678000 \\ \mathrm{C} & -1.248073000 & 4.211630000 & -1.496056000 \\ \mathrm{H} & -0.539180000 & 4.706559000 & -0.820858000 \\ \mathrm{H} & -0.888924000 & 4.354619000 & -2.527155000 \\ \mathrm{H} & -2.225832000 & 4.711876000 & -1.421884000 \\ \mathrm{C} & 1.775044000 & 3.857182000 & -3.492862000 \\ \mathrm{H} & 1.500037000 & 3.606076000 & -4.530114000 \\ \mathrm{H} & 1.212000000 & 4.757083000 & -3.198690000 \\ \mathrm{H} & 2.842855000 & 4.129078000 & -3.501822000 \\ \mathrm{C} & 2.400906000 & 1.459822000 & -2.966019000 \\ \mathrm{H} & 2.202699000 & 0.573901000 & -2.346358000 \\ \mathrm{H} & 2.226612000 & 1.191599000 & -4.021261000 \\ \mathrm{H} & 3.468333000 & 1.714189000 & -2.858968000 \\ \mathrm{H} & -4.874811000 & -0.703298000 & 3.134916000 \\ \mathrm{C} & 0.524034000 & 2.241352000 & 3.711977000 \\ \mathrm{H} & 1.189366000 & 1.376747000 & 3.561599000 \\ \mathrm{H} & 1.099710000 & 3.024986000 & 4.230930000 \\ \mathrm{H} & -0.286354000 & 1.940808000 & 4.396125000\end{array}$

Table S22. Geometry optimized structure of $\left\{\left[^{i} \operatorname{Pr}_{2} \mathrm{NN}\right] \mathrm{Cu}{ }^{\mathrm{III}}\left(\mathrm{C} \equiv \mathrm{CAr}^{\mathrm{Cl}}\right)(\mathrm{NCMe})\right\}^{+}$at BP86/6-311+G(d) level of theory (multiplicity = 3).
$\begin{array}{llll}\mathrm{Cu} & -0.590497000 & -0.126100000 & -0.082009000\end{array}$
$\mathrm{N} \quad-0.544171000 \quad-2.027760000 \quad 0.540705000$
$\mathrm{N} \quad-2.337843000 \quad 0.281407000 \quad 0.754079000$
C $\quad-2.906766000 \quad-0.466389000 \quad 1.694552000$
$\begin{array}{llll}\text { C } & -2.475026000 & -1.794249000 & 1.967706000\end{array}$
$\begin{array}{llll}\text { C } & -1.407744000 & -2.549266000 & 1.404471000\end{array}$
$\mathrm{H} \quad-3.069729000 \quad-2.327088000 \quad 2.715482000$
C $\quad 0.580150000 \quad-2.767966000 \quad 0.045104000$
C $\quad 0.489333000 \quad-3.265768000 \quad-1.292539000$
C $\quad 1.768591000 \quad-2.920835000 \quad 0.821030000$
$\begin{array}{llll}\text { C } & 1.594583000 & -3.934035000 & -1.832995000\end{array}$
$\begin{array}{llll}\text { C } & 2.848069000 & -3.575761000 & 0.197307000\end{array}$ 


\begin{tabular}{|c|c|c|c|}
\hline $\mathrm{H}$ & 706000 & -4.340708000 & \\
\hline $\mathrm{H}$ & 3.781388000 & -3.686211000 & O 750703000 \\
\hline & 2.776339000 & -4.079343000 & \\
\hline & 3.641782000 & -4.581079000 & \\
\hline & 2.054092000 & -2.474428000 & \\
\hline & 3.068115000 & -2.035320000 & 2.2 \\
\hline & -0.796490000 & -3.15 & \\
\hline & -1.418342000 & -2.363721000 & -1.6 \\
\hline & 973000 & 1.570 & \\
\hline & -2.251114000 & 2.726325000 & 0.9 \\
\hline & 957000 & 1.683 & -0.5 \\
\hline & -2.710960000 & 3.989 & \\
\hline & -4.30 & 2.985 & \\
\hline & -2.26 & 4.88 & \\
\hline & 098000 & 3.09 & -1 \\
\hline & -3.72 & 4.12 & \\
\hline & -4.059975000 & 5.12 & -0.6 \\
\hline & -4.73 & 0.55 & -1.2 \\
\hline & -4.939758000 & 0.9 & -2.2 \\
\hline & -1.172407000 & 2.6 & \\
\hline & -0.8 & 1.5 & \\
\hline & 3.6217480 & & \\
\hline & 4.4 & 1.75 & \\
\hline & 871000 & 1.96 & -1.5 \\
\hline C & 5.76 & 2.28 & \\
\hline & 5.456051 & 2.48 & -1. \\
\hline & 6.246 & 2.64 & -0.5 \\
\hline & -0.995 & 0.54 & -2.0 \\
\hline & -0.888333000 & 1.22 & -3.0 \\
\hline & -0.748673000 & 2.07 & \\
\hline $\mathrm{H}$ & -0.6598050 & & \\
\hline $\mathrm{H}$ & -1.6251390 & 2.72 & -4.2 \\
\hline $\mathrm{H}$ & 0.153985000 & 2.6948 & -4.0 \\
\hline & 1.170 & 0.58 & $-0 .($ \\
\hline 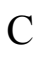 & 2.3213040 & 1.05 & -0.1 \\
\hline $\mathrm{Cl}$ & 3.212931 & 1.75 & -2.9 \\
\hline $\mathrm{Cl}_{1}$ & 3.886583000 & 1.316076000 & \\
\hline $\mathrm{H}$ & 5.837024 & 2.76 & -2 \\
\hline $\mathrm{H}$ & 7.254922000 & 3.056505000 & -0.6 \\
\hline $\mathrm{H}$ & 6.375359000 & 2.40 & \\
\hline 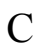 & -4.068396000 & 0.03 & \\
\hline & -3.76 & 0.07 & 3.5 \\
\hline$\Pi$ & -4.410643000 & 1.026740000 & 2.22518200 \\
\hline & -4.916505000 & -0.669528000 & $2.4755270 \mathrm{C}$ \\
\hline 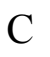 & -1.750859000 & 3.007700000 & 3.43826700 \\
\hline $\mathrm{H}$ & -0.980860000 & 2.894830000 & 4.21900300 \\
\hline
\end{tabular}




$\begin{array}{lrrr}\mathrm{H} & -2.095771000 & 4.054744000 & 3.459927000 \\ \mathrm{H} & -2.607968000 & 2.373172000 & 3.714015000 \\ \mathrm{C} & 0.053747000 & 3.515313000 & 1.730632000 \\ \mathrm{H} & 0.478203000 & 3.269883000 & 0.745497000 \\ \mathrm{H} & -0.193239000 & 4.589627000 & 1.740963000 \\ \mathrm{H} & 0.841075000 & 3.355401000 & 2.484442000 \\ \mathrm{C} & -6.109553000 & 0.417845000 & -0.542399000 \\ \mathrm{H} & -6.011913000 & 0.006157000 & 0.474590000 \\ \mathrm{H} & -6.631625000 & 1.383827000 & -0.462636000 \\ \mathrm{H} & -6.753980000 & -0.268445000 & -1.116159000 \\ \mathrm{C} & -4.095729000 & -0.825365000 & -1.436320000 \\ \mathrm{H} & -3.090714000 & -0.760138000 & -1.875092000 \\ \mathrm{H} & -4.030400000 & -1.408286000 & -0.505525000 \\ \mathrm{C} & -1.603258000 & -4.469326000 & -2.049361000 \\ \mathrm{H} & -1.854546000 & -4.753591000 & -1.015178000 \\ \mathrm{H} & -2.546586000 & -4.374160000 & -2.612182000 \\ \mathrm{H} & -1.031540000 & -5.302257000 & -2.491142000 \\ \mathrm{C} & -1.321527000 & -3.994477000 & 1.854350000 \\ \mathrm{H} & -0.535009000 & -4.549677000 & 1.330150000 \\ \mathrm{H} & -1.122580000 & -4.046568000 & 2.937258000 \\ \mathrm{H} & -2.287823000 & -4.496372000 & 1.684858000 \\ \mathrm{C} & 1.178106000 & -1.397615000 & 2.930474000 \\ \mathrm{H} & 1.679434000 & -1.057219000 & 3.851386000 \\ \mathrm{H} & 1.040322000 & -0.519210000 & 2.284805000 \\ \mathrm{H} & 0.189441000 & -1.774772000 & 3.235501000 \\ \mathrm{C} & 2.165317000 & -3.706180000 & 3.202691000 \\ \mathrm{H} & 2.874204000 & -4.456530000 & 2.821029000 \\ \mathrm{H} & 2.511363000 & -3.395015000 & 4.202065000 \\ \mathrm{H} & 1.192005000 & -4.206830000 & 3.330312000 \\ \mathrm{H} & -4.726739000 & -1.405217000 & -2.130066000 \\ \mathrm{H} & -0.542071000 & -2.744507000 & -3.573991000 \\ \mathrm{H} & 0.085268000 & -1.842206000 & -3.640246000 \\ \mathrm{H} & -0.044292000 & -3.543542000 & -4.146927000 \\ & -1.499164000 & -2.538556000 & -4.080749000\end{array}$

Table S23. Geometry optimized structure of $\left\{\left[^{i} \mathrm{Pr}_{2} \mathrm{NN}\right] \mathrm{Cu}{ }^{\mathrm{II}}\left(\mathrm{C} \equiv \mathrm{CAr}^{\mathrm{Cl}}\right)(\mathrm{Ph})\right\}^{-}$at $\mathrm{BP} 86 / 6-$ $311+\mathrm{G}(\mathrm{d})$ level of theory.

$\begin{array}{lrrr}\mathrm{Cu} & -0.460798000 & 0.345410000 & 0.201976000 \\ \mathrm{~N} & -2.282917000 & 0.324406000 & -0.757074000 \\ \mathrm{~N} & 0.039714000 & 2.248491000 & -0.565686000 \\ \mathrm{C} & -0.893783000 & 3.078409000 & -1.036937000 \\ \mathrm{C} & -2.242681000 & 2.704522000 & -1.276758000 \\ \mathrm{C} & -2.857236000 & 1.426268000 & -1.253203000 \\ \mathrm{H} & -2.876477000 & 3.507011000 & -1.663231000 \\ \mathrm{C} & -2.949538000 & -0.935581000 & -0.906217000 \\ \mathrm{C} & -2.535421000 & -1.803051000 & -1.961817000\end{array}$




\begin{tabular}{|c|c|c|c|}
\hline $\mathrm{C}$ & -3.977390000 & -1.356039000 & -0.011106000 \\
\hline $\mathrm{C}$ & -3.192098000 & -3.032423000 & -2.133137000 \\
\hline $\mathrm{C}$ & -4.593609000 & -2.601872000 & -0.223049000 \\
\hline $\mathrm{H}$ & -2.882813000 & -3.693263000 & -2.950302000 \\
\hline $\mathrm{H}$ & -5.382460000 & -2.928010000 & 0.464478000 \\
\hline $\mathrm{C}$ & -4.222487000 & -3.436196000 & -1.279745000 \\
\hline $\mathrm{H}$ & -4.720415000 & -4.401096000 & -1.427325000 \\
\hline $\mathrm{C}$ & -4.406572000 & -0.531710000 & 1.201936000 \\
\hline $\mathrm{H}$ & -3.853897000 & 0.420785000 & 1.175475000 \\
\hline $\mathrm{C}$ & -1.412790000 & -1.418267000 & -2.924347000 \\
\hline $\mathrm{H}$ & -0.898116000 & -0.546506000 & -2.488489000 \\
\hline $\mathrm{C}$ & 1.405148000 & 2.677247000 & -0.584024000 \\
\hline $\mathrm{C}$ & 2.001017000 & 3.288588000 & 0.556337000 \\
\hline $\mathrm{C}$ & 2.193668000 & 2.437266000 & -1.748218000 \\
\hline $\mathrm{C}$ & 3.350244000 & 3.676961000 & 0.493457000 \\
\hline $\mathrm{C}$ & 3.538191000 & 2.842913000 & -1.758425000 \\
\hline $\mathrm{H}$ & 3.809521000 & 4.147609000 & 1.370488000 \\
\hline $\mathrm{H}$ & 4.145758000 & 2.656469000 & -2.651640000 \\
\hline $\mathrm{C}$ & 4.121299000 & 3.469068000 & -0.653117000 \\
\hline $\mathrm{H}$ & 5.172304000 & 3.777922000 & -0.679925000 \\
\hline $\mathrm{C}$ & 1.626040000 & 1.723925000 & -2.975219000 \\
\hline $\mathrm{H}$ & 0.564213000 & 1.511368000 & -2.771983000 \\
\hline $\mathrm{C}$ & 1.227923000 & 3.506199000 & 1.854193000 \\
\hline $\mathrm{H}$ & 0.201693000 & 3.142085000 & 1.694405000 \\
\hline $\mathrm{C}$ & -0.440112000 & -0.310819000 & 3.161948000 \\
\hline $\mathrm{C}$ & -1.778504000 & 1.593561000 & 2.515996000 \\
\hline $\mathrm{C}$ & -0.780680000 & -0.093143000 & 4.508832000 \\
\hline $\mathrm{C}$ & -2.118635000 & 1.818651000 & 3.862937000 \\
\hline $\mathrm{C}$ & -1.619611000 & 0.973366000 & 4.865350000 \\
\hline $\mathrm{C}$ & -0.932759000 & 0.529625000 & 2.146657000 \\
\hline $\mathrm{H}$ & -2.776735000 & 2.656854000 & 4.126925000 \\
\hline $\mathrm{H}$ & -1.883445000 & 1.144641000 & 5.915609000 \\
\hline $\mathrm{H}$ & -0.384146000 & -0.761327000 & 5.283973000 \\
\hline $\mathrm{C}$ & -0.527795000 & 4.511656000 & -1.408792000 \\
\hline $\mathrm{H}$ & -0.003072000 & 5.017191000 & -0.583151000 \\
\hline $\mathrm{H}$ & 0.162067000 & 4.533314000 & -2.268688000 \\
\hline $\mathrm{H}$ & -1.420975000 & 5.098163000 & -1.670445000 \\
\hline $\mathrm{C}$ & 1.146282000 & 4.994346000 & 2.255720000 \\
\hline $\mathrm{H}$ & 2.144956000 & 5.415349000 & 2.468396000 \\
\hline $\mathrm{H}$ & 0.690089000 & 5.612372000 & 1.464133000 \\
\hline $\mathrm{H}$ & 0.536358000 & 5.113085000 & 3.168241000 \\
\hline $\mathrm{C}$ & 1.830123000 & 2.670591000 & 3.002502000 \\
\hline $\mathrm{H}$ & 2.851233000 & 3.009339000 & 3.254582000 \\
\hline $\mathrm{H}$ & 1.208201000 & 2.754603000 & 3.909633000 \\
\hline $\mathrm{H}$ & 1.880658000 & 1.606630000 & 2.729693000 \\
\hline $\mathrm{C}$ & 1.694839000 & 2.595659000 & -4.247384000 \\
\hline
\end{tabular}




\begin{tabular}{|c|c|c|c|}
\hline $\mathrm{H}$ & 1.172834000 & 3.557860000 & -4.114914000 \\
\hline $\mathrm{H}$ & 2.738117000 & 2.821237000 & -4.530213000 \\
\hline $\mathrm{H}$ & 1.226872000 & 2.074451000 & -5.100975000 \\
\hline $\mathrm{C}$ & 2.333561000 & 0.371808000 & -3.202070000 \\
\hline $\mathrm{H}$ & 2.239942000 & -0.275083000 & -2.316552000 \\
\hline $\mathrm{H}$ & 1.894399000 & -0.156295000 & -4.066960000 \\
\hline $\mathrm{C}$ & -1.970258000 & -0.996017000 & -4.301260000 \\
\hline $\mathrm{H}$ & -1.151775000 & -0.696668000 & -4.979495000 \\
\hline $\mathrm{H}$ & -2.517658000 & -1.826488000 & -4.782179000 \\
\hline $\mathrm{H}$ & -2.663977000 & -0.143564000 & -4.216377000 \\
\hline $\mathrm{C}$ & -4.251000000 & 1.354749000 & -1.868027000 \\
\hline $\mathrm{H}$ & -4.343480000 & 0.496191000 & -2.551483000 \\
\hline $\mathrm{H}$ & -5.022141000 & 1.217793000 & -1.092988000 \\
\hline $\mathrm{H}$ & -4.487425000 & 2.273855000 & -2.423954000 \\
\hline $\mathrm{C}$ & -5.918380000 & -0.215024000 & 1.209736000 \\
\hline $\mathrm{H}$ & -6.523897000 & -1.130673000 & 1.328412000 \\
\hline $\mathrm{H}$ & -6.165840000 & 0.452162000 & 2.053745000 \\
\hline $\mathrm{H}$ & -6.251140000 & 0.277608000 & 0.281377000 \\
\hline $\mathrm{C}$ & -4.019144000 & -1.247409000 & 2.513224000 \\
\hline $\mathrm{H}$ & -4.561672000 & -2.203973000 & 2.618833000 \\
\hline $\mathrm{H}$ & -2.941196000 & -1.458096000 & 2.547884000 \\
\hline $\mathrm{H}$ & -4.263268000 & -0.618554000 & 3.385913000 \\
\hline $\mathrm{H}$ & 3.409459000 & 0.510034000 & -3.408232000 \\
\hline $\mathrm{C}$ & -0.366117000 & -2.539003000 & -3.074994000 \\
\hline $\mathrm{H}$ & -0.789843000 & -3.444236000 & -3.544712000 \\
\hline $\mathrm{H}$ & 0.467709000 & -2.199525000 & -3.712305000 \\
\hline $\mathrm{H}$ & 0.052060000 & -2.819333000 & -2.096608000 \\
\hline $\mathrm{C}$ & 0.915814000 & -0.973989000 & 0.256080000 \\
\hline $\mathrm{C}$ & 1.799438000 & -1.839734000 & 0.389796000 \\
\hline $\mathrm{C}$ & 2.795794000 & -2.826930000 & 0.537173000 \\
\hline $\mathrm{C}$ & 4.193057000 & -2.527012000 & 0.511288000 \\
\hline $\mathrm{C}$ & 2.490770000 & -4.209568000 & 0.730048000 \\
\hline $\mathrm{Cl}$ & 0.802871000 & -4.703027000 & 0.785557000 \\
\hline $\mathrm{Cl}$ & 4.693197000 & -0.857512000 & 0.285875000 \\
\hline $\mathrm{C}$ & 5.185223000 & -3.498436000 & 0.659390000 \\
\hline $\mathrm{C}$ & 3.468313000 & -5.195165000 & 0.880016000 \\
\hline $\mathrm{H}$ & 3.163292000 & -6.234755000 & 1.024549000 \\
\hline $\mathrm{C}$ & 4.822868000 & -4.839088000 & 0.844342000 \\
\hline $\mathrm{H}$ & 5.595164000 & -5.605584000 & 0.961064000 \\
\hline $\mathrm{H}$ & 6.235955000 & -3.198675000 & 0.630291000 \\
\hline $\mathrm{H}$ & 0.221012000 & -1.143195000 & 2.899031000 \\
\hline $\mathrm{H}$ & -2.183685000 & 2.267487000 & 1.749015000 \\
\hline
\end{tabular}

Table S24. Geometry optimized structure of $\left[{ }^{i} \mathrm{Pr}_{2} \mathrm{NN}\right] \mathrm{Cu}^{\mathrm{III}}\left(\mathrm{C} \equiv \mathrm{CAr}^{\mathrm{Cl}}\right)(\mathrm{Ph})$ at $\mathrm{BP} 86 / 6$ $311+\mathrm{G}(\mathrm{d})$ level of theory with charge $=0$, multiplicity $=1$.

$\mathrm{Cu} \quad 0.578465000 \quad-0.313721000 \quad 0.217495000$ 


\begin{tabular}{|c|c|c|c|}
\hline $\mathrm{N}$ & 2.274442000 & -0.608677000 & -0.692567000 \\
\hline $\mathrm{N}$ & -0.195367000 & -2.173258000 & -0.116208000 \\
\hline $\mathrm{C}$ & 0.621981000 & -3.185828000 & -0.426084000 \\
\hline $\mathrm{C}$ & 1.984612000 & -3.017239000 & -0.773523000 \\
\hline $\mathrm{C}$ & 2.712660000 & -1.833318000 & -1.022849000 \\
\hline $\mathrm{H}$ & 2.514515000 & -3.939117000 & -1.026988000 \\
\hline $\mathrm{C}$ & 3.013717000 & 0.578853000 & -1.053551000 \\
\hline $\mathrm{C}$ & 2.463985000 & 1.388300000 & -2.094885000 \\
\hline $\mathrm{C}$ & 4.235430000 & 0.951278000 & -0.417534000 \\
\hline $\mathrm{C}$ & 3.147413000 & 2.556144000 & -2.470216000 \\
\hline $\mathrm{C}$ & 4.865121000 & 2.137993000 & -0.832626000 \\
\hline $\mathrm{H}$ & 2.739801000 & 3.178604000 & -3.271831000 \\
\hline $\mathrm{H}$ & 5.800007000 & 2.439129000 & -0.349595000 \\
\hline $\mathrm{C}$ & 4.333522000 & 2.940244000 & -1.842677000 \\
\hline $\mathrm{H}$ & 4.844484000 & 3.860840000 & -2.142715000 \\
\hline $\mathrm{C}$ & 4.921000000 & 0.140478000 & 0.684006000 \\
\hline $\mathrm{H}$ & 4.299713000 & -0.744204000 & 0.900104000 \\
\hline $\mathrm{C}$ & 1.219321000 & 0.982334000 & -2.884180000 \\
\hline $\mathrm{H}$ & 0.659237000 & 0.246687000 & -2.283265000 \\
\hline $\mathrm{C}$ & -1.603585000 & -2.455196000 & -0.017364000 \\
\hline $\mathrm{C}$ & -2.196333000 & -2.589707000 & 1.267824000 \\
\hline $\mathrm{C}$ & -2.387456000 & -2.586662000 & -1.197209000 \\
\hline $\mathrm{C}$ & -3.560634000 & -2.917034000 & 1.342344000 \\
\hline $\mathrm{C}$ & -3.749636000 & -2.901185000 & -1.063562000 \\
\hline $\mathrm{H}$ & -4.026826000 & -3.039461000 & 2.325546000 \\
\hline $\mathrm{H}$ & -4.367401000 & -3.001445000 & -1.961980000 \\
\hline $\mathrm{C}$ & -4.335590000 & -3.082772000 & 0.192264000 \\
\hline $\mathrm{H}$ & -5.397055000 & -3.338482000 & 0.273935000 \\
\hline $\mathrm{C}$ & -1.814448000 & -2.328956000 & -2.590774000 \\
\hline $\mathrm{H}$ & -0.718409000 & -2.250288000 & -2.499641000 \\
\hline $\mathrm{C}$ & -1.402974000 & -2.405608000 & 2.559419000 \\
\hline $\mathrm{H}$ & -0.378550000 & -2.111700000 & 2.282094000 \\
\hline $\mathrm{C}$ & 1.342680000 & 1.582196000 & 2.454451000 \\
\hline $\mathrm{C}$ & 2.038902000 & -0.754339000 & 2.621669000 \\
\hline $\mathrm{C}$ & 1.774041000 & 1.794845000 & 3.777426000 \\
\hline $\mathrm{C}$ & 2.458329000 & -0.527530000 & 3.947754000 \\
\hline $\mathrm{C}$ & 2.322629000 & 0.742091000 & 4.523225000 \\
\hline $\mathrm{C}$ & 1.482101000 & 0.307282000 & 1.906848000 \\
\hline $\mathrm{H}$ & 2.897365000 & -1.352662000 & 4.519258000 \\
\hline $\mathrm{H}$ & 2.647434000 & 0.912220000 & 5.554588000 \\
\hline $\mathrm{H}$ & 1.657309000 & 2.787633000 & 4.225145000 \\
\hline $\mathrm{C}$ & 0.098944000 & -4.613298000 & -0.466840000 \\
\hline $\mathrm{H}$ & -0.601384000 & -4.807832000 & 0.358278000 \\
\hline $\mathrm{H}$ & -0.456110000 & -4.802782000 & -1.399980000 \\
\hline $\mathrm{H}$ & 0.927229000 & -5.334551000 & -0.414491000 \\
\hline $\mathrm{C}$ & -1.306312000 & -3.714533000 & 3.372094000 \\
\hline
\end{tabular}




\begin{tabular}{|c|c|c|c|}
\hline $\mathrm{H}$ & -2.301213000 & -4.065180000 & 3.695510000 \\
\hline $\mathrm{H}$ & -0.842661000 & -4.527930000 & 2.789502000 \\
\hline $\mathrm{H}$ & -0.698328000 & -3.562162000 & 4.280155000 \\
\hline $\mathrm{C}$ & -1.986411000 & -1.265576000 & 3.419336000 \\
\hline $\mathrm{H}$ & -3.000680000 & -1.506402000 & 3.781073000 \\
\hline $\mathrm{H}$ & -1.351097000 & -1.087853000 & 4.303273000 \\
\hline $\mathrm{H}$ & -2.044815000 & -0.328570000 & 2.845282000 \\
\hline $\mathrm{C}$ & -2.117487000 & -3.464249000 & -3.590358000 \\
\hline $\mathrm{H}$ & -1.780616000 & -4.446239000 & -3.219351000 \\
\hline $\mathrm{H}$ & -3.196236000 & -3.546111000 & -3.804210000 \\
\hline $\mathrm{H}$ & -1.609811000 & -3.275547000 & -4.551340000 \\
\hline $\mathrm{C}$ & -2.329093000 & -0.977898000 & -3.134603000 \\
\hline $\mathrm{H}$ & -2.096915000 & -0.155364000 & -2.441159000 \\
\hline $\mathrm{H}$ & -1.874400000 & -0.752989000 & -4.114996000 \\
\hline $\mathrm{C}$ & 1.629539000 & 0.286276000 & -4.201706000 \\
\hline $\mathrm{H}$ & 0.737673000 & -0.038044000 & -4.764000000 \\
\hline $\mathrm{H}$ & 2.204335000 & 0.971170000 & -4.848598000 \\
\hline $\mathrm{H}$ & 2.255817000 & -0.602131000 & -4.018296000 \\
\hline $\mathrm{C}$ & 4.029469000 & -2.029317000 & -1.756699000 \\
\hline $\mathrm{H}$ & 4.416760000 & -1.095115000 & -2.181316000 \\
\hline $\mathrm{H}$ & 4.794826000 & -2.439087000 & -1.078033000 \\
\hline $\mathrm{H}$ & 3.895774000 & -2.757811000 & -2.570987000 \\
\hline $\mathrm{C}$ & 6.328761000 & -0.339773000 & 0.257340000 \\
\hline $\mathrm{H}$ & 7.022789000 & 0.511109000 & 0.154311000 \\
\hline $\mathrm{H}$ & 6.751115000 & -1.012343000 & 1.023181000 \\
\hline $\mathrm{H}$ & 6.327316000 & -0.876132000 & -0.702754000 \\
\hline $\mathrm{C}$ & 5.048105000 & 0.952001000 & 1.990699000 \\
\hline $\mathrm{H}$ & 5.723382000 & 1.814182000 & 1.857245000 \\
\hline $\mathrm{H}$ & 4.077787000 & 1.333793000 & 2.333352000 \\
\hline $\mathrm{H}$ & 5.471144000 & 0.324434000 & 2.792558000 \\
\hline $\mathrm{H}$ & -3.423762000 & -0.998132000 & -3.269800000 \\
\hline $\mathrm{C}$ & 0.263233000 & 2.157353000 & -3.159312000 \\
\hline $\mathrm{H}$ & 0.700821000 & 2.902411000 & -3.845099000 \\
\hline $\mathrm{H}$ & -0.659269000 & 1.786258000 & -3.634213000 \\
\hline $\mathrm{H}$ & -0.022123000 & 2.669285000 & -2.228063000 \\
\hline $\mathrm{C}$ & -1.010262000 & 0.759282000 & 0.222752000 \\
\hline $\mathrm{C}$ & -1.937643000 & 1.576263000 & 0.241940000 \\
\hline $\mathrm{C}$ & -2.981246000 & 2.533340000 & 0.196972000 \\
\hline $\mathrm{C}$ & -4.354969000 & 2.165345000 & 0.112626000 \\
\hline $\mathrm{C}$ & -2.722585000 & 3.933441000 & 0.242887000 \\
\hline $\mathrm{Cl}$ & -1.060730000 & 4.497486000 & 0.346858000 \\
\hline $\mathrm{Cl}$ & -4.771998000 & 0.465577000 & 0.050010000 \\
\hline $\mathrm{C}$ & -5.384403000 & 3.108877000 & 0.078358000 \\
\hline $\mathrm{C}$ & -3.739040000 & 4.889728000 & 0.209021000 \\
\hline $\mathrm{H}$ & -3.482520000 & 5.950733000 & 0.248179000 \\
\hline $\mathrm{C}$ & -5.073006000 & 4.472540000 & 0.126475000 \\
\hline
\end{tabular}




$\begin{array}{rrrr}\mathrm{H} & -5.874822000 & 5.216002000 & 0.099974000 \\ \mathrm{H} & -6.421069000 & 2.770493000 & 0.014398000 \\ \mathrm{H} & 0.876966000 & 2.391138000 & 1.887470000 \\ \mathrm{H} & 2.155523000 & -1.749891000 & 2.178518000\end{array}$

Table S25. Geometry optimized structure of $\left[{ }^{i} \mathrm{Pr}_{2} \mathrm{NN}\right] \mathrm{Cu}^{\mathrm{I}}\left({ }^{\mathrm{Cl}} \mathrm{ArC} \equiv \mathrm{C}-\mathrm{Ph}\right)$ at $\mathrm{BP} 86 / 6$ $311+\mathrm{G}(\mathrm{d})$ level of theory.

$\begin{array}{lrrr}\mathrm{Cu} & -0.157377000 & 0.320241000 & -0.121892000 \\ \mathrm{~N} & -1.779094000 & 1.353811000 & -0.575316000 \\ \mathrm{~N} & 1.158149000 & 1.791187000 & -0.246463000 \\ \mathrm{C} & 0.785249000 & 3.047484000 & -0.543709000 \\ \mathrm{C} & -0.527539000 & 3.420119000 & -0.901290000 \\ \mathrm{C} & -1.712292000 & 2.657305000 & -0.905687000 \\ \mathrm{H} & -0.653385000 & 4.471257000 & -1.169980000 \\ \mathrm{C} & -3.073250000 & 0.726692000 & -0.626300000 \\ \mathrm{C} & -3.444243000 & 0.008154000 & -1.800017000 \\ \mathrm{C} & -3.967898000 & 0.829315000 & 0.474401000 \\ \mathrm{C} & -4.728194000 & -0.561777000 & -1.858818000 \\ \mathrm{C} & -5.232129000 & 0.222271000 & 0.369479000 \\ \mathrm{H} & -5.036842000 & -1.101056000 & -2.760039000 \\ \mathrm{H} & -5.930655000 & 0.291148000 & 1.210139000 \\ \mathrm{C} & -5.621369000 & -0.456742000 & -0.787349000 \\ \mathrm{H} & -6.617653000 & -0.906094000 & -0.854887000 \\ \mathrm{C} & -3.601429000 & 1.575885000 & 1.758440000 \\ \mathrm{H} & -2.639521000 & 2.085280000 & 1.584373000 \\ \mathrm{C} & -2.493188000 & -0.127940000 & -2.991231000 \\ \mathrm{H} & -1.473672000 & 0.065300000 & -2.614329000 \\ \mathrm{C} & 2.513381000 & 1.586290000 & 0.192344000 \\ \mathrm{C} & 2.859074000 & 1.861346000 & 1.545089000 \\ \mathrm{C} & 3.487471000 & 1.109822000 & -0.730971000 \\ \mathrm{C} & 4.184119000 & 1.634925000 & 1.955622000 \\ \mathrm{C} & 4.799597000 & 0.911682000 & -0.266826000 \\ \mathrm{H} & 4.467414000 & 1.834946000 & 2.994206000 \\ \mathrm{H} & 5.566217000 & 0.548708000 & -0.956828000 \\ \mathrm{C} & 5.150374000 & 1.166937000 & 1.062897000 \\ \mathrm{H} & 6.178882000 & 1.002366000 & 1.400788000 \\ \mathrm{C} & 3.128080000 & 0.836780000 & -2.194998000 \\ \mathrm{H} & 2.126433000 & 0.367520000 & -2.195005000 \\ \mathrm{C} & 1.837780000 & 2.397121000 & 2.553005000 \\ \mathrm{H} & 0.960856000 & 2.750709000 & 1.987189000 \\ \mathrm{C} & -2.855718000 & -2.511247000 & 0.662258000 \\ \mathrm{C} & -1.306417000 & -2.783007000 & 2.522964000 \\ \mathrm{C} & -3.800346000 & -3.279606000 & 1.350583000 \\ \mathrm{C} & -2.261603000 & -3.540297000 & 3.205737000 \\ \mathrm{C} & -3.512010000 & -3.794500000 & 2.621952000 \\ & -1.590953000 & -2.261374000 & 1.235254000\end{array}$




\begin{tabular}{|c|c|c|c|}
\hline $\mathrm{H}$ & -2.025558000 & -3.939042000 & 4.197507000 \\
\hline $\mathrm{H}$ & -4.257834000 & -4.390350000 & 3.157380000 \\
\hline $\mathrm{H}$ & -4.773511000 & -3.469262000 & 0.887881000 \\
\hline $\mathrm{C}$ & 1.789982000 & 4.188879000 & -0.465938000 \\
\hline $\mathrm{H}$ & 1.843202000 & 4.579498000 & 0.564661000 \\
\hline $\mathrm{H}$ & 2.807211000 & 3.881127000 & -0.742050000 \\
\hline $\mathrm{H}$ & 1.480156000 & 5.020912000 & -1.115001000 \\
\hline $\mathrm{C}$ & 2.377394000 & 3.593709000 & 3.364415000 \\
\hline $\mathrm{H}$ & 3.177407000 & 3.293103000 & 4.061723000 \\
\hline $\mathrm{H}$ & 2.787859000 & 4.382722000 & 2.713453000 \\
\hline $\mathrm{H}$ & 1.571118000 & 4.039239000 & 3.971227000 \\
\hline $\mathrm{C}$ & 1.336508000 & 1.291700000 & 3.505932000 \\
\hline $\mathrm{H}$ & 2.166589000 & 0.871161000 & 4.098921000 \\
\hline $\mathrm{H}$ & 0.590381000 & 1.696655000 & 4.211129000 \\
\hline $\mathrm{H}$ & 0.868306000 & 0.464702000 & 2.950612000 \\
\hline $\mathrm{C}$ & 3.019263000 & 2.128304000 & -3.039082000 \\
\hline $\mathrm{H}$ & 2.203399000 & 2.782829000 & -2.704218000 \\
\hline $\mathrm{H}$ & 3.961571000 & 2.701965000 & -3.002993000 \\
\hline $\mathrm{H}$ & 2.824024000 & 1.874309000 & -4.095129000 \\
\hline $\mathrm{C}$ & 4.103042000 & -0.125544000 & -2.897609000 \\
\hline $\mathrm{H}$ & 4.290607000 & -1.035982000 & -2.309321000 \\
\hline $\mathrm{H}$ & 3.687829000 & -0.432347000 & -3.871584000 \\
\hline $\mathrm{C}$ & -2.786341000 & 0.924839000 & -4.083345000 \\
\hline $\mathrm{H}$ & -2.093740000 & 0.803986000 & -4.933798000 \\
\hline $\mathrm{H}$ & -3.814895000 & 0.818064000 & -4.469541000 \\
\hline $\mathrm{H}$ & -2.672472000 & 1.952491000 & -3.706733000 \\
\hline $\mathrm{C}$ & -2.977367000 & 3.422095000 & -1.269209000 \\
\hline $\mathrm{H}$ & -3.694939000 & 2.812242000 & -1.834893000 \\
\hline $\mathrm{H}$ & -3.498872000 & 3.757360000 & -0.357036000 \\
\hline $\mathrm{H}$ & -2.732010000 & 4.318539000 & -1.856458000 \\
\hline $\mathrm{C}$ & -4.644890000 & 2.650582000 & 2.133498000 \\
\hline $\mathrm{H}$ & -5.609271000 & 2.199378000 & 2.422117000 \\
\hline $\mathrm{H}$ & -4.292757000 & 3.243536000 & 2.994441000 \\
\hline $\mathrm{H}$ & -4.844446000 & 3.346600000 & 1.303117000 \\
\hline $\mathrm{C}$ & -3.403520000 & 0.603319000 & 2.940662000 \\
\hline $\mathrm{H}$ & -4.336063000 & 0.060518000 & 3.170306000 \\
\hline $\mathrm{H}$ & -2.628950000 & -0.147662000 & 2.726753000 \\
\hline $\mathrm{H}$ & -3.105870000 & 1.154423000 & 3.849240000 \\
\hline $\mathrm{H}$ & 5.076014000 & 0.354622000 & -3.101439000 \\
\hline $\mathrm{C}$ & -2.502031000 & -1.541040000 & -3.606600000 \\
\hline $\mathrm{H}$ & -3.460480000 & -1.775680000 & -4.099886000 \\
\hline $\mathrm{H}$ & -1.719440000 & -1.621826000 & -4.379492000 \\
\hline $\mathrm{H}$ & -2.310676000 & -2.320308000 & -2.852040000 \\
\hline $\mathrm{C}$ & -0.538938000 & -1.539766000 & 0.552602000 \\
\hline $\mathrm{C}$ & 0.675487000 & -1.475467000 & 0.185812000 \\
\hline $\mathrm{C}$ & 1.899608000 & -2.173478000 & -0.127964000 \\
\hline
\end{tabular}




$\begin{array}{lrrr}\mathrm{C} & 2.886006000 & -2.462542000 & 0.849617000 \\ \mathrm{C} & 2.131498000 & -2.712533000 & -1.420183000 \\ \mathrm{Cl} & 0.957428000 & -2.431979000 & -2.694911000 \\ \mathrm{Cl} & 2.662759000 & -1.898852000 & 2.494440000 \\ \mathrm{C} & 4.021970000 & -3.224832000 & 0.565709000 \\ \mathrm{C} & 3.253950000 & -3.488610000 & -1.719595000 \\ \mathrm{H} & 3.376815000 & -3.888266000 & -2.728489000 \\ \mathrm{C} & 4.202701000 & -3.738587000 & -0.722434000 \\ \mathrm{H} & 5.086869000 & -4.340470000 & -0.950403000 \\ \mathrm{H} & 4.748555000 & -3.419048000 & 1.357545000 \\ \mathrm{H} & -3.094877000 & -2.106155000 & -0.322431000 \\ \mathrm{H} & -0.327134000 & -2.591513000 & 2.969936000\end{array}$

Table S26. Geometry optimized structure of $\left[{ }^{i} \mathrm{Pr}_{2} \mathrm{NN}\right] \mathrm{Cu}^{\mathrm{I}}$ at $\mathrm{BP} 86 / 6-311+\mathrm{G}(\mathrm{d})$ level of theory.

$\begin{array}{lrrr}\mathrm{Cu} & -0.000004000 & 0.000033000 & -0.526583000 \\ \mathrm{~N} & -1.605824000 & 0.041882000 & 0.433907000 \\ \mathrm{~N} & 1.605829000 & -0.041935000 & 0.433880000 \\ \mathrm{C} & 1.313692000 & -0.050486000 & 1.755139000 \\ \mathrm{C} & 0.000015000 & -0.000142000 & 2.307307000 \\ \mathrm{C} & -1.313669000 & 0.050270000 & 1.755164000 \\ \mathrm{H} & 0.000023000 & -0.000209000 & 3.399895000 \\ \mathrm{C} & -2.960772000 & 0.083233000 & -0.033905000 \\ \mathrm{C} & -3.536937000 & 1.337718000 & -0.377879000 \\ \mathrm{C} & -3.680153000 & -1.126497000 & -0.227094000 \\ \mathrm{C} & -4.838031000 & 1.354387000 & -0.906372000 \\ \mathrm{C} & -4.978174000 & -1.054574000 & -0.762295000 \\ \mathrm{H} & -5.299721000 & 2.310091000 & -1.173146000 \\ \mathrm{H} & -5.546187000 & -1.977364000 & -0.92056900 \\ \mathrm{C} & -5.559565000 & 0.171089000 & -1.097226000 \\ \mathrm{C} & 2.960771000 & -0.083228000 & -0.033956000 \\ \mathrm{C} & 3.536933000 & -1.337672000 & -0.378087000 \\ \mathrm{C} & 3.680147000 & 1.126526000 & -0.227012000 \\ \mathrm{C} & 4.838019000 & -1.354276000 & -0.906600000 \\ \mathrm{C} & 4.978161000 & 1.054668000 & -0.762239000 \\ \mathrm{H} & 5.299708000 & -2.309948000 & -1.173493000 \\ \mathrm{H} & 5.546170000 & 1.977477000 & -0.920411000 \\ \mathrm{C} & 5.559550000 & -0.170955000 & -1.097323000 \\ \mathrm{C} & -2.466066000 & 0.117089000 & 2.743595000 \\ \mathrm{H} & -3.124954000 & -0.761545000 & 2.646485000 \\ \mathrm{H} & -2.104361000 & 0.164705000 & 3.779516000 \\ \mathrm{H} & -3.099263000 & 0.999494000 & 2.553891000 \\ \mathrm{C} & 2.466103000 & -0.117427000 & 2.743546000 \\ \mathrm{H} & 3.124993000 & 0.761217000 & 2.646531000 \\ \mathrm{H} & 2.104412000 & -0.165163000 & 3.779466000 \\ \mathrm{H} & 3.099293000 & -0.999812000 & 2.553729000\end{array}$




$\begin{array}{lrrr}\mathrm{C} & -3.053241000 & -2.486585000 & 0.076473000 \\ \mathrm{H} & -2.139658000 & -2.308930000 & 0.667292000 \\ \mathrm{C} & -2.733582000 & 2.629861000 & -0.235929000 \\ \mathrm{H} & -1.978496000 & 2.465924000 & 0.551675000 \\ \mathrm{C} & 2.733582000 & -2.629833000 & -0.236280000 \\ \mathrm{H} & 1.978503000 & -2.465990000 & 0.551351000 \\ \mathrm{C} & 3.053238000 & 2.486577000 & 0.076725000 \\ \mathrm{H} & 2.139657000 & 2.308850000 & 0.667527000 \\ \mathrm{H} & -6.573600000 & 0.205594000 & -1.508497000 \\ \mathrm{H} & 6.573579000 & -0.205409000 & -1.508612000 \\ \mathrm{C} & 2.622440000 & 3.185042000 & -1.231811000 \\ \mathrm{H} & 1.918126000 & 2.561042000 & -1.806090000 \\ \mathrm{H} & 2.127890000 & 4.148596000 & -1.020571000 \\ \mathrm{H} & 3.492459000 & 3.387102000 & -1.879601000 \\ \mathrm{C} & 3.972814000 & 3.403456000 & 0.907733000 \\ \mathrm{H} & 4.306075000 & 2.913816000 & 1.837404000 \\ \mathrm{H} & 4.875634000 & 3.702401000 & 0.349108000 \\ \mathrm{H} & 3.442144000 & 4.329648000 & 1.185829000 \\ \mathrm{C} & -3.580007000 & 3.845858000 & 0.183270000 \\ \mathrm{H} & -4.278744000 & 4.163111000 & -0.609040000 \\ \mathrm{H} & -4.172868000 & 3.638088000 & 1.089010000 \\ \mathrm{H} & -2.926594000 & 4.708087000 & 0.397345000 \\ \mathrm{C} & -1.967974000 & 2.924620000 & -1.545011000 \\ \mathrm{H} & -1.299994000 & 2.086470000 & -1.814101000 \\ \mathrm{H} & -2.666727000 & 3.076969000 & -2.385047000 \\ \mathrm{H} & -1.346458000 & 3.830847000 & -1.447461000 \\ \mathrm{C} & -3.972811000 & -3.403567000 & 0.907373000 \\ \mathrm{H} & -4.875633000 & -3.702448000 & 0.348715000 \\ \mathrm{H} & -3.442138000 & -4.329791000 & 1.185355000 \\ \mathrm{H} & -4.306071000 & -2.914041000 & 1.837105000 \\ \mathrm{C} & -2.622449000 & -3.184889000 & -1.232151000 \\ \mathrm{H} & -2.127897000 & -4.148469000 & -1.021032000 \\ \mathrm{H} & -3.492472000 & -3.386870000 & -1.879961000 \\ \mathrm{H} & -1.918140000 & -2.560818000 & -1.806357000 \\ \mathrm{H} & 1.967961000 & -2.924441000 & -1.545389000 \\ \mathrm{H} & 1.3466705000 & -3.076689000 & -2.385450000 \\ \mathrm{H} & 3.580014000 & -3.845878000 & 0.182768000 \\ \mathrm{H} & -172883000 & -3.638212000 & 1.088527000 \\ \mathrm{H} & -3.8305000 & -4.708133000 & 0.396749000 \\ \mathrm{H} & -4.163035000 & -0.609586000\end{array}$

Table S27. Geometry optimized structure of $\left[{ }^{i} \operatorname{Pr}_{2} \mathrm{NN}\right] \mathrm{Cu}^{\mathrm{I}}\left(\mathrm{C}_{6} \mathrm{H}_{6}\right)$ at $\mathrm{BP} 86 / 6-311+\mathrm{G}(\mathrm{d})$ level of theory with charge $=0$, multiplicity $=1$.

$\begin{array}{llll}\mathrm{Cu} & 0.000336000 & 0.213694000 & 0.381140000\end{array}$ 


\begin{tabular}{|c|c|c|c|}
\hline $\mathrm{N}$ & -1.511453000 & 0.136786000 & -0.868666000 \\
\hline $\mathrm{N}$ & 1.512695000 & 0.136720000 & -0.867987000 \\
\hline $\mathrm{C}$ & 1.287714000 & 0.110344000 & -2.192542000 \\
\hline $\mathrm{C}$ & 0.001079000 & 0.126193000 & -2.780113000 \\
\hline $\mathrm{C}$ & -1.285819000 & 0.110184000 & -2.193101000 \\
\hline $\mathrm{H}$ & 0.001311000 & 0.116544000 & -3.873383000 \\
\hline $\mathrm{C}$ & -2.848687000 & 0.082077000 & -0.356259000 \\
\hline $\mathrm{C}$ & -3.517794000 & -1.167053000 & -0.222436000 \\
\hline $\mathrm{C}$ & -3.466895000 & 1.284870000 & 0.098255000 \\
\hline $\mathrm{C}$ & -4.791655000 & -1.187477000 & 0.372946000 \\
\hline $\mathrm{C}$ & -4.743446000 & 1.207625000 & 0.679882000 \\
\hline $\mathrm{H}$ & -5.315497000 & -2.143009000 & 0.484977000 \\
\hline $\mathrm{H}$ & -5.232480000 & 2.121910000 & 1.030285000 \\
\hline $\mathrm{C}$ & -5.406412000 & -0.016213000 & 0.822258000 \\
\hline $\mathrm{H}$ & -6.400276000 & -0.054430000 & 1.279973000 \\
\hline $\mathrm{C}$ & 2.849665000 & 0.082606000 & -0.354883000 \\
\hline $\mathrm{C}$ & 3.466886000 & 1.285645000 & 0.100374000 \\
\hline $\mathrm{C}$ & 3.519514000 & -1.166140000 & -0.221094000 \\
\hline $\mathrm{C}$ & 4.743238000 & 1.209045000 & 0.682515000 \\
\hline $\mathrm{C}$ & 4.793126000 & -1.185920000 & 0.374842000 \\
\hline $\mathrm{H}$ & 5.231508000 & 2.123528000 & 1.033463000 \\
\hline $\mathrm{H}$ & 5.317533000 & -2.141150000 & 0.486802000 \\
\hline $\mathrm{C}$ & 5.406952000 & -0.014397000 & 0.824756000 \\
\hline $\mathrm{H}$ & 6.400647000 & -0.052108000 & 1.282878000 \\
\hline $\mathrm{C}$ & -0.715419000 & 0.595064000 & 2.331995000 \\
\hline $\mathrm{C}$ & -1.416155000 & -0.498941000 & 2.918817000 \\
\hline $\mathrm{C}$ & 0.716581000 & 0.590532000 & 2.332624000 \\
\hline $\mathrm{C}$ & -0.715898000 & -1.557745000 & 3.482880000 \\
\hline $\mathrm{C}$ & 1.409680000 & -0.508117000 & 2.919867000 \\
\hline $\mathrm{C}$ & 0.702120000 & -1.562382000 & 3.483339000 \\
\hline $\mathrm{H}$ & 1.241098000 & -2.395105000 & 3.946066000 \\
\hline $\mathrm{H}$ & 2.503365000 & -0.499864000 & 2.935733000 \\
\hline $\mathrm{H}$ & 1.273437000 & 1.506883000 & 2.113133000 \\
\hline $\mathrm{H}$ & -1.266019000 & 1.515032000 & 2.111779000 \\
\hline $\mathrm{H}$ & -2.509780000 & -0.483718000 & 2.933653000 \\
\hline $\mathrm{H}$ & -1.260625000 & -2.386906000 & 3.945255000 \\
\hline $\mathrm{C}$ & -2.451444000 & 0.022346000 & -3.168128000 \\
\hline $\mathrm{H}$ & -2.209373000 & 0.531465000 & -4.112951000 \\
\hline $\mathrm{H}$ & -2.667079000 & -1.031784000 & -3.413646000 \\
\hline $\mathrm{H}$ & -3.375844000 & 0.452178000 & -2.758419000 \\
\hline $\mathrm{C}$ & 2.453821000 & 0.022842000 & -3.166999000 \\
\hline $\mathrm{H}$ & 2.670198000 & -1.031258000 & -3.412004000 \\
\hline $\mathrm{H}$ & 2.211924000 & 0.531470000 & -4.112122000 \\
\hline $\mathrm{H}$ & 3.377788000 & 0.453348000 & -2.757002000 \\
\hline $\mathrm{C}$ & 2.776094000 & 2.639867000 & -0.070916000 \\
\hline $\mathrm{H}$ & 1.688705000 & 2.442734000 & -0.101108000 \\
\hline
\end{tabular}




$\begin{array}{lrrr}\mathrm{C} & 3.148515000 & 3.299747000 & -1.417926000 \\ \mathrm{H} & 2.860926000 & 2.672788000 & -2.274859000 \\ \mathrm{H} & 2.638913000 & 4.272284000 & -1.529708000 \\ \mathrm{H} & 4.235716000 & 3.479233000 & -1.479419000 \\ \mathrm{C} & 3.049449000 & 3.624085000 & 1.083240000 \\ \mathrm{H} & 4.089836000 & 3.990770000 & 1.078452000 \\ \mathrm{H} & 2.399764000 & 4.510145000 & 0.989190000 \\ \mathrm{H} & 2.864482000 & 3.172766000 & 2.072091000 \\ \mathrm{C} & 2.892983000 & -2.480704000 & -0.689661000 \\ \mathrm{H} & 1.964475000 & -2.235874000 & -1.230274000 \\ \mathrm{C} & 3.813059000 & -3.252848000 & -1.659393000 \\ \mathrm{H} & 3.290853000 & -4.135293000 & -2.066460000 \\ \mathrm{H} & 4.135194000 & -2.627469000 & -2.507385000 \\ \mathrm{H} & 4.724149000 & -3.617536000 & -1.155522000 \\ \mathrm{C} & 2.500502000 & -3.378265000 & 0.503121000 \\ \mathrm{H} & 2.026428000 & -4.310235000 & 0.150070000 \\ \mathrm{H} & 3.385477000 & -3.659801000 & 1.099647000 \\ \mathrm{H} & 1.792222000 & -2.867889000 & 1.173719000 \\ \mathrm{C} & -2.776941000 & 2.639481000 & -0.073208000 \\ \mathrm{H} & -1.689433000 & 2.443025000 & -0.103197000 \\ \mathrm{C} & -3.149537000 & 3.298914000 & -1.420386000 \\ \mathrm{H} & -4.236839000 & 3.477691000 & -1.482164000 \\ \mathrm{H} & -2.640557000 & 4.271777000 & -1.532180000 \\ \mathrm{H} & -2.861341000 & 2.672027000 & -2.277165000 \\ \mathrm{C} & -3.051181000 & 3.623705000 & 1.080744000 \\ \mathrm{H} & -2.866386000 & 3.172552000 & 2.069695000 \\ \mathrm{H} & -2.401930000 & 4.510097000 & 0.986835000 \\ \mathrm{H} & -4.091761000 & 3.989846000 & 1.075514000 \\ \mathrm{C} & -2.890214000 & -2.481341000 & -0.690371000 \\ \mathrm{H} & -1.961493000 & -2.236089000 & -1.230417000 \\ \mathrm{C} & -2.498026000 & -3.378438000 & 0.502850000 \\ \mathrm{H} & -2.023023000 & -4.310136000 & 0.150329000 \\ \mathrm{H} & -1.790665000 & -2.867412000 & 1.173926000 \\ & -3.383258000 & -3.660481000 & 1.098752000 \\ \mathrm{H} & -3.809250000 & -3.254124000 & -1.660571000 \\ \mathrm{H} & -4.131059000 & -2.629082000 & -2.508936000\end{array}$

Table S28. Geometry optimized structure of $\left[{ }^{i} \operatorname{Pr}_{2} \mathrm{NN}\right] \mathrm{Cu}^{\mathrm{I}}(\mathrm{NCMe})$ at $\mathrm{BP} 86 / 6-311+\mathrm{G}(\mathrm{d})$ level of theory with charge $=0$, multiplicity $=1$.

$\begin{array}{lrrr}\mathrm{Cu} & -0.002181000 & 0.000138000 & 0.450015000 \\ \mathrm{~N} & -1.497143000 & 0.000163000 & -0.802929000 \\ \mathrm{~N} & 1.490197000 & 0.000207000 & -0.807493000 \\ \mathrm{C} & 1.279044000 & 0.000441000 & -2.134411000 \\ \mathrm{C} & -0.006297000 & 0.000592000 & -2.727948000\end{array}$




\begin{tabular}{|c|c|c|c|}
\hline $\mathrm{C}$ & -1.289809000 & 0.000418000 & -2.130536000 \\
\hline $\mathrm{H}$ & -0.007957000 & 0.000805000 & -3.820210000 \\
\hline $\mathrm{C}$ & -2.833950000 & -0.000051000 & -0.284198000 \\
\hline $\mathrm{C}$ & -3.477588000 & -1.234870000 & 0.011335000 \\
\hline $\mathrm{C}$ & -3.478029000 & 1.234566000 & 0.011252000 \\
\hline $\mathrm{C}$ & -4.749704000 & -1.207539000 & 0.607350000 \\
\hline $\mathrm{C}$ & -4.750139000 & 1.206823000 & 0.607255000 \\
\hline $\mathrm{H}$ & -5.253168000 & -2.151891000 & 0.841870000 \\
\hline $\mathrm{H}$ & -5.253944000 & 2.151007000 & 0.841701000 \\
\hline $\mathrm{C}$ & -5.389007000 & -0.000464000 & 0.906321000 \\
\hline $\mathrm{H}$ & -6.382096000 & -0.000623000 & 1.367580000 \\
\hline $\mathrm{C}$ & 2.828180000 & -0.000011000 & -0.292108000 \\
\hline $\mathrm{C}$ & 3.472861000 & 1.234591000 & 0.002339000 \\
\hline $\mathrm{C}$ & 3.472507000 & -1.234836000 & 0.002164000 \\
\hline $\mathrm{C}$ & 4.746156000 & 1.206820000 & 0.595817000 \\
\hline $\mathrm{C}$ & 4.745809000 & -1.207515000 & 0.595649000 \\
\hline $\mathrm{H}$ & 5.250394000 & 2.151047000 & 0.829240000 \\
\hline $\mathrm{H}$ & 5.249774000 & -2.151922000 & 0.828940000 \\
\hline $\mathrm{C}$ & 5.385707000 & -0.000461000 & 0.893517000 \\
\hline $\mathrm{H}$ & 6.379797000 & -0.000636000 & 1.352631000 \\
\hline $\mathrm{C}$ & -2.485132000 & 0.000521000 & -3.068996000 \\
\hline $\mathrm{H}$ & -3.125310000 & 0.881753000 & -2.898638000 \\
\hline $\mathrm{H}$ & -2.168319000 & 0.000804000 & -4.121160000 \\
\hline $\mathrm{H}$ & -3.125133000 & -0.880923000 & -2.899059000 \\
\hline $\mathrm{C}$ & 2.471515000 & 0.000539000 & -3.076509000 \\
\hline $\mathrm{H}$ & 3.112013000 & -0.880902000 & -2.908510000 \\
\hline $\mathrm{H}$ & 2.151540000 & 0.000800000 & -4.127718000 \\
\hline $\mathrm{H}$ & 3.112181000 & 0.881783000 & -2.908120000 \\
\hline $\mathrm{C}$ & 2.793710000 & 2.575396000 & -0.275124000 \\
\hline $\mathrm{H}$ & 1.892255000 & 2.371425000 & -0.875786000 \\
\hline $\mathrm{C}$ & 3.684305000 & 3.540529000 & -1.083583000 \\
\hline $\mathrm{H}$ & 4.030403000 & 3.083657000 & -2.025072000 \\
\hline $\mathrm{H}$ & 3.126774000 & 4.457723000 & -1.339053000 \\
\hline $\mathrm{H}$ & 4.579427000 & 3.850006000 & -0.517832000 \\
\hline $\mathrm{C}$ & 2.327130000 & 3.235617000 & 1.040450000 \\
\hline $\mathrm{H}$ & 3.183734000 & 3.460629000 & 1.699142000 \\
\hline $\mathrm{H}$ & 1.798061000 & 4.183376000 & 0.840749000 \\
\hline $\mathrm{H}$ & 1.642071000 & 2.573655000 & 1.594412000 \\
\hline $\mathrm{C}$ & 2.793019000 & -2.575424000 & -0.275519000 \\
\hline $\mathrm{H}$ & 1.891385000 & -2.371083000 & -0.875782000 \\
\hline $\mathrm{C}$ & 3.683181000 & -3.540391000 & -1.084657000 \\
\hline $\mathrm{H}$ & 3.125459000 & -4.457453000 & -1.340190000 \\
\hline $\mathrm{H}$ & 4.028928000 & -3.083250000 & -2.026144000 \\
\hline $\mathrm{H}$ & 4.578513000 & -3.850118000 & -0.519374000 \\
\hline $\mathrm{C}$ & 2.326807000 & -3.236052000 & 1.039979000 \\
\hline $\mathrm{H}$ & 1.797494000 & -4.183640000 & 0.840115000 \\
\hline
\end{tabular}




$\begin{array}{lrrr}\mathrm{H} & 3.183603000 & -3.461475000 & 1.698280000 \\ \mathrm{H} & 1.642068000 & -2.574176000 & 1.594439000 \\ \mathrm{C} & -2.798846000 & 2.575145000 & -0.267223000 \\ \mathrm{H} & -1.901622000 & 2.371533000 & -0.874329000 \\ \mathrm{C} & -2.322492000 & 3.230543000 & 1.047273000 \\ \mathrm{H} & -1.634240000 & 2.565845000 & 1.594033000 \\ \mathrm{H} & -1.793819000 & 4.178450000 & 0.847196000 \\ \mathrm{H} & -3.174403000 & 3.453921000 & 1.712584000 \\ \mathrm{C} & -3.692951000 & 3.544119000 & -1.067063000 \\ \mathrm{H} & -4.046633000 & 3.090217000 & -2.007176000 \\ \mathrm{H} & -4.583368000 & 3.854451000 & -0.494408000 \\ \mathrm{H} & -3.135098000 & 4.460632000 & -1.324249000 \\ \mathrm{C} & -2.798016000 & -2.575258000 & -0.267102000 \\ \mathrm{H} & -1.900504000 & -2.371334000 & -0.873668000 \\ \mathrm{C} & -3.691568000 & -3.544131000 & -1.067691000 \\ \mathrm{H} & -4.582318000 & -3.854617000 & -0.495637000 \\ \mathrm{H} & -4.044706000 & -3.090048000 & -2.007920000 \\ \mathrm{H} & -3.133506000 & -4.460558000 & -1.324737000 \\ \mathrm{C} & -2.322272000 & -3.230943000 & 1.047469000 \\ \mathrm{H} & -1.793361000 & -4.178722000 & 0.847413000 \\ \mathrm{H} & -1.634399000 & -2.566298000 & 1.594767000 \\ \mathrm{H} & -3.174490000 & -3.454631000 & 1.712281000 \\ \mathrm{C} & 0.025881000 & 0.000085000 & 3.469060000 \\ \mathrm{~N} & 0.010081000 & 0.000093000 & 2.298837000 \\ \mathrm{C} & 0.047450000 & 0.000078000 & 4.921069000 \\ \mathrm{H} & 1.082885000 & -0.001939000 & 5.298960000 \\ \mathrm{H} & -0.468681000 & -0.891247000 & 5.312699000 \\ \mathrm{H} & -0.465145000 & 0.893480000 & 5.312607000\end{array}$

Table S29. Geometry optimized structure of ${ }^{\mathrm{C} 12} \mathrm{ArC} \equiv \mathrm{C} \cdot$ radical at $\mathrm{BP} 86 / 6-311+\mathrm{G}(\mathrm{d})$ level of theory with charge $=0$, multiplicity $=2$.
C $\quad-0.000053000$
3.035889000
0.195456000
C $\quad-0.000021000$
1.798486000
$-0.105889000$
$\mathrm{Cl} 2.743600000$
0.486065000
$-0.030443000$
$\mathrm{Cl}-2.743598000$
0.486019000
$-0.030451000$
C $\quad-0.000026000$
0.403294000
$-0.036926000$
C $\quad 1.223789000$
$-0.355292000$
$-0.009836000$
C $\quad 1.222601000$
$-1.747329000$
0.030055000
$\mathrm{H} \quad 2.169919000$
$-2.290086000$
0.046661000
C $\quad 0.000045000$
$-2.435140000$
0.050185000
$\mathrm{H} \quad 0.000047000$
$-3.528455000$
0.082062000
C $\quad-1.222534000$
$-1.747362000$
0.030075000
$\mathrm{H} \quad-2.169819000$
$-2.290180000$
0.046729000
C $\quad-1.223832000$
$-0.355331000$
$-0.009826000$ 
Table S30. Geometry optimized structure of ${ }^{\mathrm{Cl} 2} \mathrm{ArC} \equiv \mathrm{C}^{-}$at $\mathrm{BP} 86 / 6-311+\mathrm{G}(\mathrm{d})$ level of theory with charge $=-1$, multiplicity $=1$.

$\begin{array}{lrrr}\mathrm{C} & 0.000097000 & 3.101645000 & 0.000415000 \\ \mathrm{C} & 0.000024000 & 1.838835000 & 0.000165000 \\ \mathrm{Cl} & 2.755395000 & 0.471327000 & -0.000152000 \\ \mathrm{Cl} & -2.755401000 & 0.471385000 & -0.000150000 \\ \mathrm{C} & 0.000005000 & 0.434466000 & -0.000113000 \\ \mathrm{C} & 1.196278000 & -0.359398000 & -0.000063000 \\ \mathrm{C} & 1.209683000 & -1.754599000 & 0.000109000 \\ \mathrm{H} & 2.168653000 & -2.280743000 & 0.000178000 \\ \mathrm{C} & -0.000039000 & -2.464598000 & 0.000168000 \\ \mathrm{H} & -0.000056000 & -3.559252000 & 0.000451000 \\ \mathrm{C} & -1.209723000 & -1.754568000 & 0.000106000 \\ \mathrm{H} & -2.168716000 & -2.280673000 & 0.000143000 \\ \mathrm{C} & -1.196288000 & -0.359356000 & -0.000060000\end{array}$

Table S31. Geometry optimized structure of ${ }^{\mathrm{C} 12} \mathrm{ArC} \equiv \mathrm{C}-\mathrm{C} \equiv \mathrm{Ar}^{\mathrm{Cl} 2}$ at $\mathrm{BP} 86 / 6-311+\mathrm{G}(\mathrm{d})$ level of theory with charge $=0$, multiplicity $=1$.

$\begin{array}{lrrr}\mathrm{C} & -0.674195000 & -0.000234000 & -0.000012000 \\ \mathrm{C} & -1.904843000 & -0.000161000 & -0.000008000 \\ \mathrm{Cl} & -3.212040000 & 2.739987000 & 0.000378000 \\ \mathrm{Cl} & -3.212503000 & -2.740089000 & -0.000388000 \\ \mathrm{C} & -3.314195000 & -0.000042000 & 0.000002000 \\ \mathrm{C} & -4.063910000 & 1.211588000 & 0.000171000 \\ \mathrm{C} & -5.459457000 & 1.216786000 & 0.000178000 \\ \mathrm{H} & -5.994123000 & 2.169056000 & 0.000312000 \\ \mathrm{C} & -6.152655000 & 0.000197000 & 0.000013000 \\ \mathrm{H} & -7.246254000 & 0.000290000 & 0.000016000 \\ \mathrm{C} & -5.459662000 & -1.216509000 & -0.000160000 \\ \mathrm{H} & -5.994489000 & -2.168688000 & -0.000293000 \\ \mathrm{C} & -4.064114000 & -1.211546000 & -0.000163000 \\ \mathrm{C} & 1.904843000 & -0.000207000 & -0.000008000 \\ \mathrm{Cl} & 3.212589000 & -2.740093000 & 0.000377000 \\ \mathrm{Cl} & 3.211954000 & 2.739982000 & -0.000388000 \\ \mathrm{C} & 3.314195000 & -0.000043000 & 0.000006000 \\ \mathrm{C} & 4.064152000 & -1.211523000 & 0.000173000 \\ \mathrm{C} & 5.459700000 & -1.216442000 & 0.000177000 \\ \mathrm{H} & 5.994557000 & -2.168605000 & 0.000309000 \\ \mathrm{C} & 6.152655000 & 0.000286000 & 0.000012000 \\ \mathrm{H} & 7.246254000 & 0.000412000 & 0.000013000 \\ \mathrm{C} & 5.459419000 & 1.216853000 & -0.000160000 \\ \mathrm{H} & 5.994056000 & 2.169139000 & -0.000295000 \\ \mathrm{C} & 4.063872000 & 1.211611000 & -0.000161000 \\ \mathrm{C} & 0.674195000 & -0.000277000 & -0.000013000\end{array}$


Table S32. Geometry optimized structure of $\mathrm{Ph}^{-}$at BP86/6-311+G(d) level of theory with charge $=-1$, multiplicity $=1$.

$\begin{array}{rrrr}\mathrm{C} & 0.000000000 & 0.000000000 & -1.373780000 \\ \mathrm{C} & 0.000000000 & 1.206592000 & -0.652665000 \\ \mathrm{C} & 0.000000000 & -1.206592000 & -0.652665000 \\ \mathrm{H} & 0.000000000 & 2.166941000 & -1.198324000 \\ \mathrm{H} & 0.000000000 & -2.166941000 & -1.198324000 \\ \mathrm{C} & 0.000000000 & 1.182058000 & 0.758112000 \\ \mathrm{C} & 0.000000000 & -1.182058000 & 0.758112000 \\ \mathrm{H} & 0.000000000 & 2.168269000 & 1.262052000 \\ \mathrm{H} & 0.00000000 & -2.168269000 & 1.262052000 \\ \mathrm{C} & 0.000000000 & 0.000000000 & 1.553725000 \\ \mathrm{H} & 0.000000000 & 0.000000000 & -2.472492000\end{array}$

Table S33. Geometry optimized structure of ${ }^{\mathrm{C} 12} \mathrm{ArC} \equiv \mathrm{C}-\mathrm{Ph}$ at $\mathrm{BP} 86 / 6-311+\mathrm{G}(\mathrm{d})$ level of theory with charge $=0$, multiplicity $=1$.

$\begin{array}{lrrr}\mathrm{C} & -1.103692000 & 0.000285000 & -0.000004000 \\ \mathrm{C} & 0.119929000 & 0.000261000 & -0.000002000 \\ \mathrm{Cl} & 1.439673000 & -2.743409000 & 0.000001000 \\ \mathrm{Cl} & 1.440568000 & 2.743506000 & 0.000005000 \\ \mathrm{C} & 1.533318000 & 0.000032000 & 0.000002000 \\ \mathrm{C} & 2.285981000 & -1.208267000 & 0.000004000 \\ \mathrm{C} & 3.681874000 & -1.215631000 & 0.000009000 \\ \mathrm{H} & 4.215056000 & -2.168762000 & 0.000010000 \\ \mathrm{C} & 4.376856000 & -0.000430000 & 0.000012000 \\ \mathrm{H} & 5.470440000 & -0.000607000 & 0.000016000 \\ \mathrm{C} & 3.682269000 & 1.214997000 & 0.000010000 \\ \mathrm{H} & 4.215760000 & 2.167955000 & 0.000013000 \\ \mathrm{C} & 2.286373000 & 1.208087000 & 0.000006000 \\ \mathrm{C} & -3.244524000 & 1.220485000 & -0.000049000 \\ \mathrm{C} & -4.641527000 & 1.214122000 & -0.000051000 \\ \mathrm{H} & -5.186275000 & 2.163317000 & -0.000084000 \\ \mathrm{C} & -5.344994000 & 0.000003000 & -0.000011000 \\ \mathrm{H} & -6.439277000 & -0.000070000 & -0.000012000 \\ \mathrm{C} & -4.641367000 & -1.214024000 & 0.000032000 \\ \mathrm{H} & -5.185990000 & -2.163291000 & 0.000063000 \\ \mathrm{C} & -3.244364000 & -1.220202000 & 0.000034000 \\ \mathrm{C} & -2.524378000 & 0.000190000 & -0.000006000 \\ \mathrm{H} & -2.692022000 & -2.163718000 & 0.000067000 \\ \mathrm{H} & -2.692308000 & 2.164074000 & -0.000080000\end{array}$

Table S34. Geometry optimized structure of $\mathrm{C}_{6} \mathrm{H}_{6}$ at $\mathrm{BP} 86 / 6-311+\mathrm{G}(\mathrm{d})$ level of theory with charge $=0$, multiplicity $=1$.
C $\quad 0.000000000$
1.402376000
0.000000000
C 1.214494000
0.701188000
0.000000000 


$\begin{array}{rrrr}\mathrm{C} & -1.214494000 & 0.701188000 & 0.000000000 \\ \mathrm{H} & 2.162584000 & 1.248568000 & 0.000000000 \\ \mathrm{H} & -2.162584000 & 1.248568000 & 0.000000000 \\ \mathrm{C} & 1.214493000 & -0.701188000 & 0.000000000 \\ \mathrm{C} & -1.214493000 & -0.701188000 & 0.000000000 \\ \mathrm{H} & 2.162583000 & -1.248568000 & 0.000000000 \\ \mathrm{H} & -2.162583000 & -1.248568000 & 0.000000000 \\ \mathrm{C} & 0.000000000 & -1.402376000 & 0.000000000 \\ \mathrm{H} & 0.000000000 & -2.497137000 & 0.000000000 \\ \mathrm{H} & 0.000000000 & 2.497136000 & 0.000000000\end{array}$

Table S35. Geometry optimized structure of $\mathrm{MeCN}$ at BP86/6-311+G(d) level of theory with charge $=0$, multiplicity $=1$.

$\begin{array}{lrrr}\mathrm{C} & 0.000000000 & 0.000000000 & 0.277991000 \\ \mathrm{~N} & 0.000000000 & 0.000000000 & 1.443854000 \\ \mathrm{C} & 0.000000000 & 0.000000000 & -1.180009000 \\ \mathrm{H} & 0.000000000 & 1.031424000 & -1.564957000 \\ \mathrm{H} & -0.893240000 & -0.515712000 & -1.564957000 \\ \mathrm{H} & 0.893240000 & -0.515712000 & -1.564957000\end{array}$

\section{References for Computational Details}

(22) M. J. Frisch, G. W. Trucks, H. B. Schlegel, G. E. Scuseria, M. A. Robb, J. R. Cheeseman, G. Scalmani, V. Barone, G. A. Petersson, H. Nakatsuji, X. Li, M. Caricato, A. V. Marenich, J. Bloino, B. G. Janesko, R. Gomperts, B. Mennucci, H. P. Hratchian, J. V. Ortiz, A. F. Izmaylov, J. L. Sonnenberg, D. Williams-Young, F. Ding, F. Lipparini, F. Egidi, J. Goings, B. Peng, A. Petrone, T. Henderson, D. Ranasinghe, V. G. Zakrzewski, J. Gao, N. Rega, G. Zheng, W. Liang, M. Hada, M. Ehara, K. Toyota, R. Fukuda, J. Hasegawa, M. Ishida, T. Nakajima, Y. Honda, O. Kitao, H. Nakai, T. Vreven, K. Throssell, J. A. Montgomery, Jr., J. E. Peralta, F. Ogliaro, M. J. Bearpark, J. J. Heyd, E. N. Brothers, K. N. Kudin, V. N. Staroverov, T. A. Keith, R. Kobayashi, J. Normand, K. Raghavachari, A. P. Rendell, J. C. Burant, S. S. Iyengar, J. Tomasi, M. Cossi, J. M. Millam, M. Klene, C. Adamo, R. Cammi, J. W. Ochterski, R. L. Martin, K. Morokuma, O. Farkas, J. B. Foresman, D. J. Fox, (Gaussian, Inc., Wallingford, CT, Revision B.01, 2016).

(23) ChemCraft 1.6 [http://www.chemcraftprog.com]

(24) E. F. Pettersen, T. D. Goddard, C. C. Huang, G. S. Couch, D. M. Greenblatt, E. C. Meng, T. E. J. Ferrin, Comput. Chem. 2004, 25, 1605-1612.

(25) C. F. Macrae, P. R. Edgington, P. McCabe, E. Pidcock, G. P. Shields, R. Taylor, M. Towler, J. van de Streek, J. Appl. Cryst. 2006, 39, 453-457.

(26) (a) A.D. Becke, Phys. Rev. A 1988, 38, 3098-3100. (b) J. P. Perdew, Phys. Rev. B 1986, 33, 8822-8824. 
(27) A. D. McLean, G. S. Chandler, J. Chem. Phys. 1980, 72, 5639-5648.

(28) K. Raghavachari, J. S. Binkley, R. Seeger, J. A. Pople, J. Chem. Phys. 1980, 72, 650-654.

(29) A. J. H. Wachters, J. Chem. Phys. 1970, 52, 1033-1036.

(30) P. J. Hay, J. Chem. Phys. 1977, 66, 4377-4384.

(31) K. Raghavachari, G. W. Trucks, J. Chem. Phys. 1989, 91, 1062-1065.

(32) R. C. Binning Jr., L. A. Curtiss, J. Comp. Chem. 1990, 11, 1206-1216.

(33) M. P. McGrath, L. Radom, J. Chem. Phys. 1991, 94, 511-516.

(34) L. A. Curtiss, M. P. McGrath, J.-P. Blaudeau, N. E. Davis, R. C. Binning Jr., L. Radom, J. Chem. Phys. 1995, 103, 6104-6113.

(35) A. V. Marenich, C. J. Cramer, D. G. Truhlar, J. Phys. Chem. B 2009, 113, 63786396.

(36) S. Grimme, S. Ehrlich, L. Goerigk, J. Comp. Chem. 2011, 32, 1456-1465. 


\section{Spectral Data}

${ }^{1} \mathrm{H}$ NMR of 1-ethynyl-2-bromobenzene $\left(\mathrm{CDCl}_{3}, 400 \mathrm{MHz}\right)$
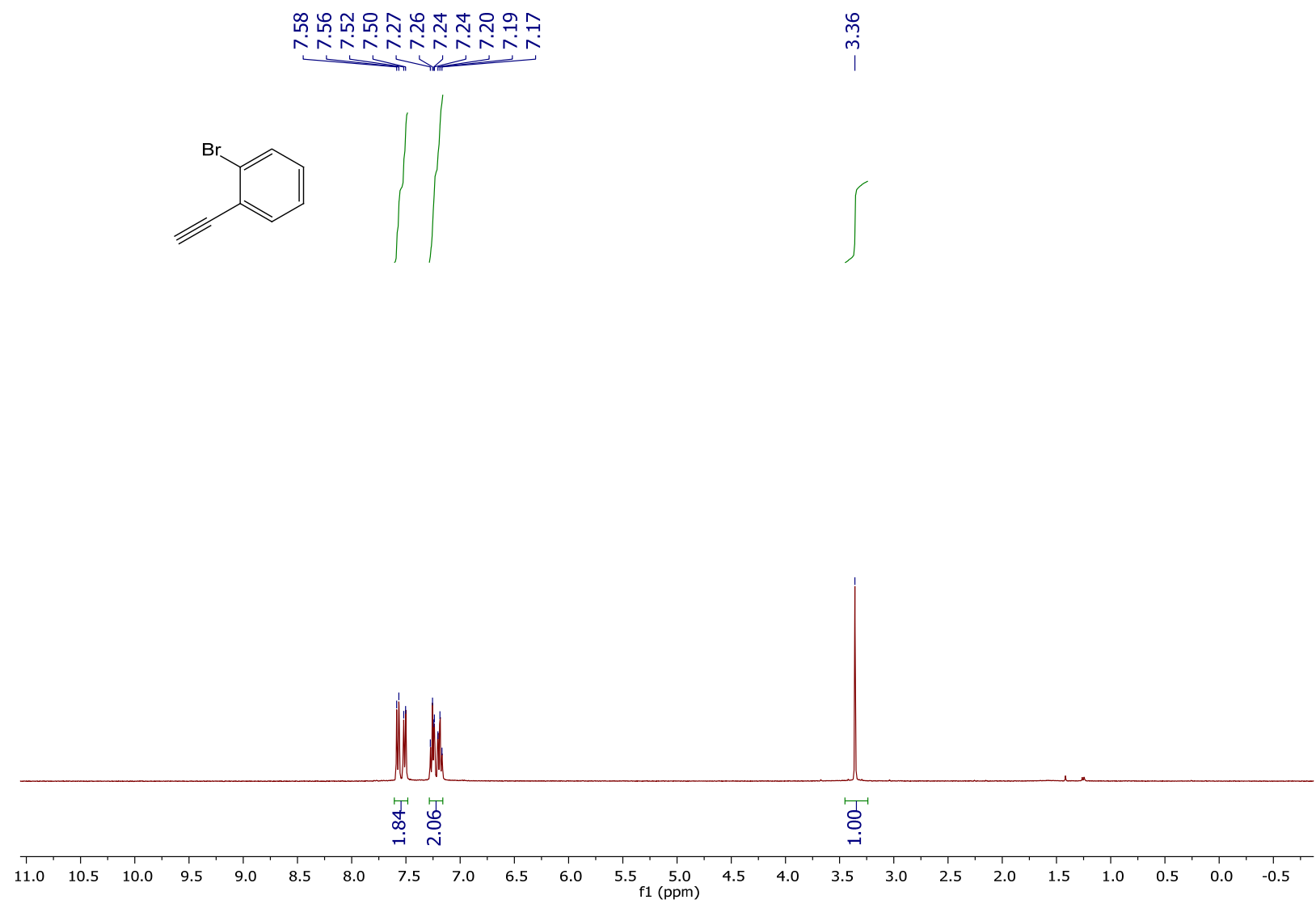
${ }^{13} \mathrm{C}\left\{{ }^{1} \mathrm{H}\right\}$ NMR of 1-ethynyl-2-bromobenzene $\left(\mathrm{CDCl}_{3}, 100 \mathrm{MHz}\right)$
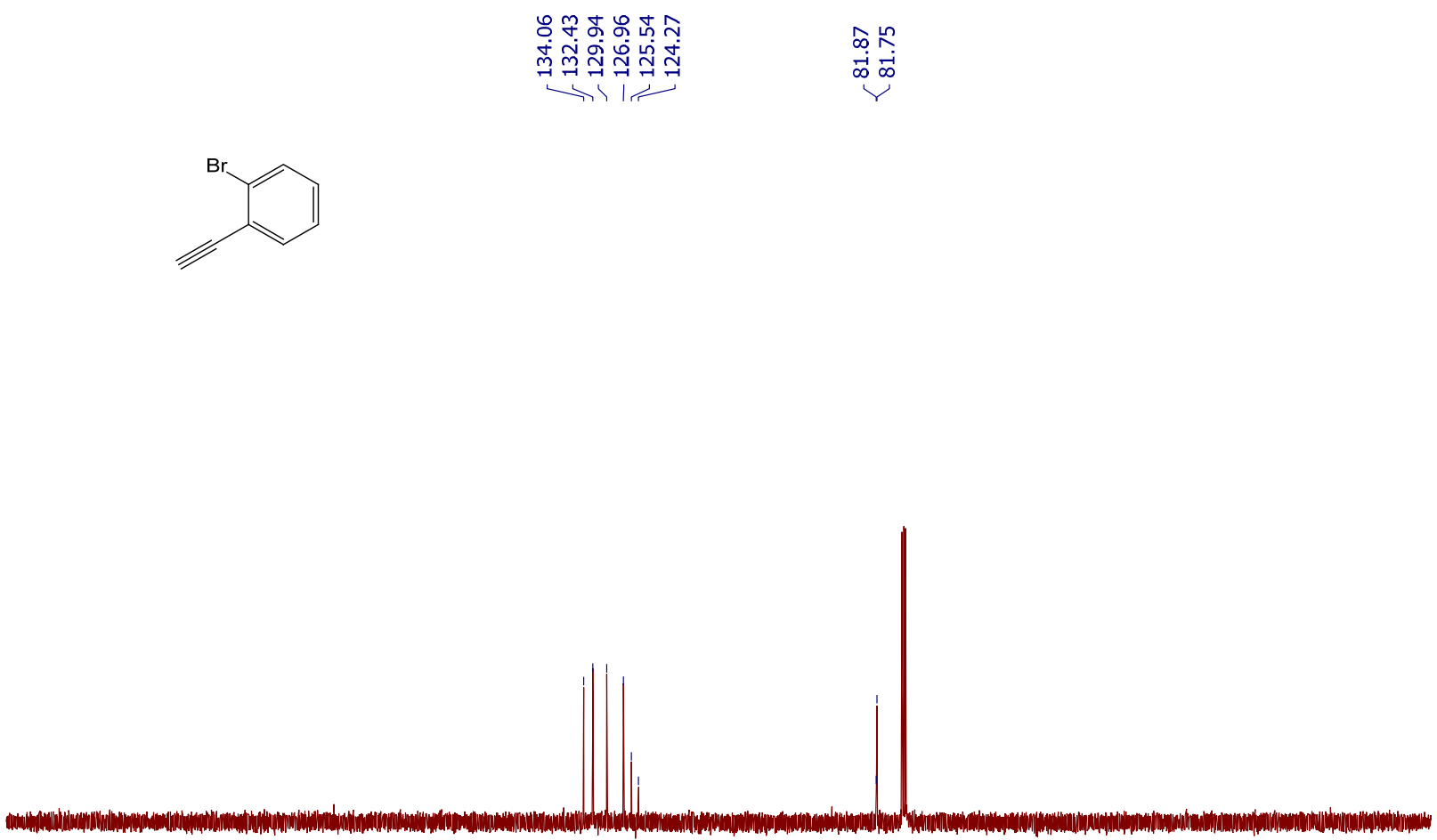

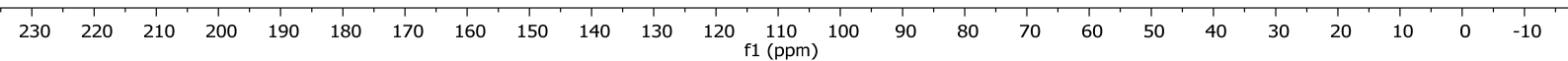


${ }^{1} \mathrm{H}$ NMR of 1-ethynyl-2,6-dichlorobenzene ( $\left.\mathrm{CDCl}_{3}, 400 \mathrm{MHz}\right)$
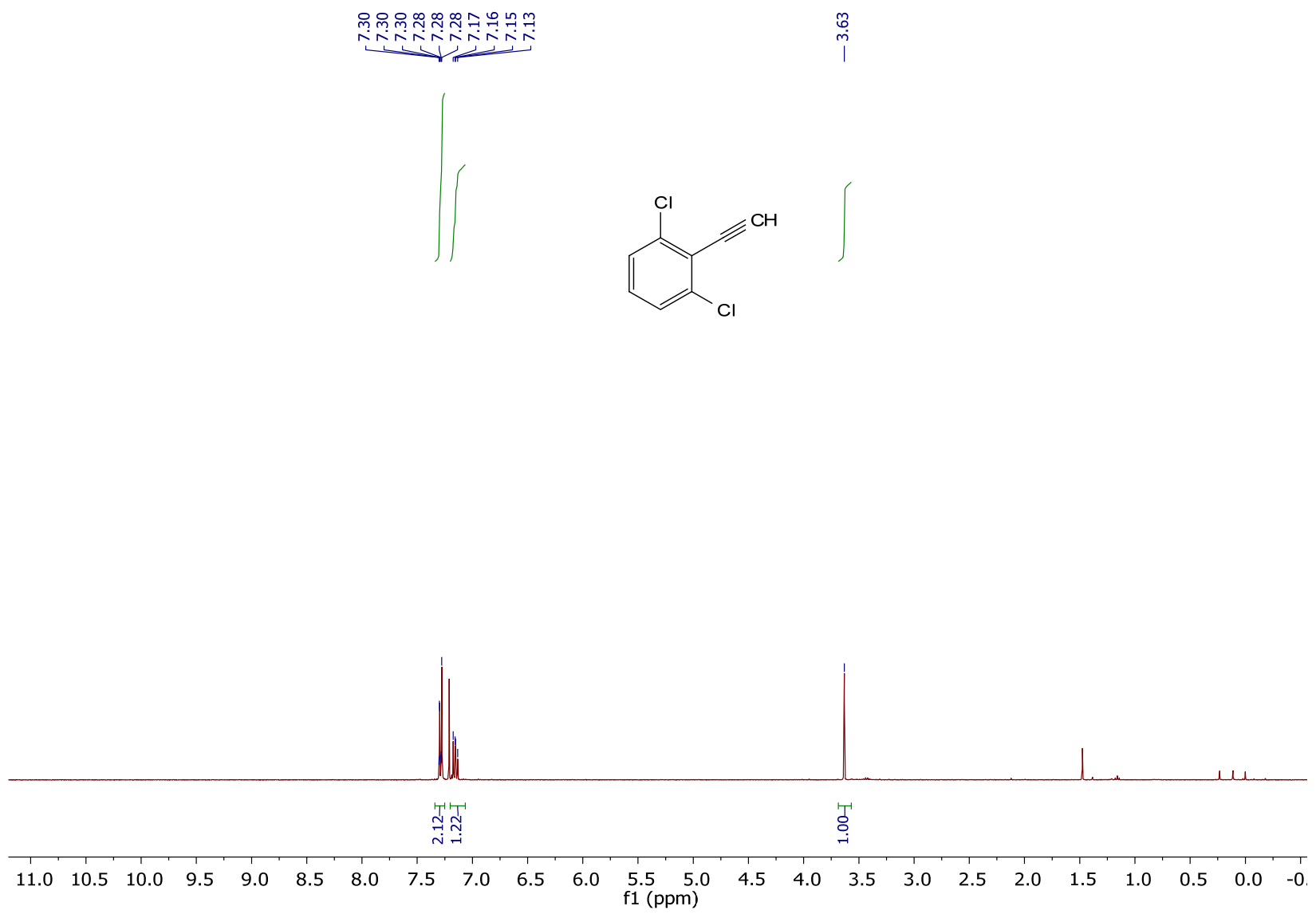
${ }^{13} \mathrm{C}\left\{{ }^{1} \mathrm{H}\right\}$ NMR of 1-ethynyl-2,6-dichlorobenzene $\left(\mathrm{CDCl}_{3}, 100 \mathrm{MHz}\right)$

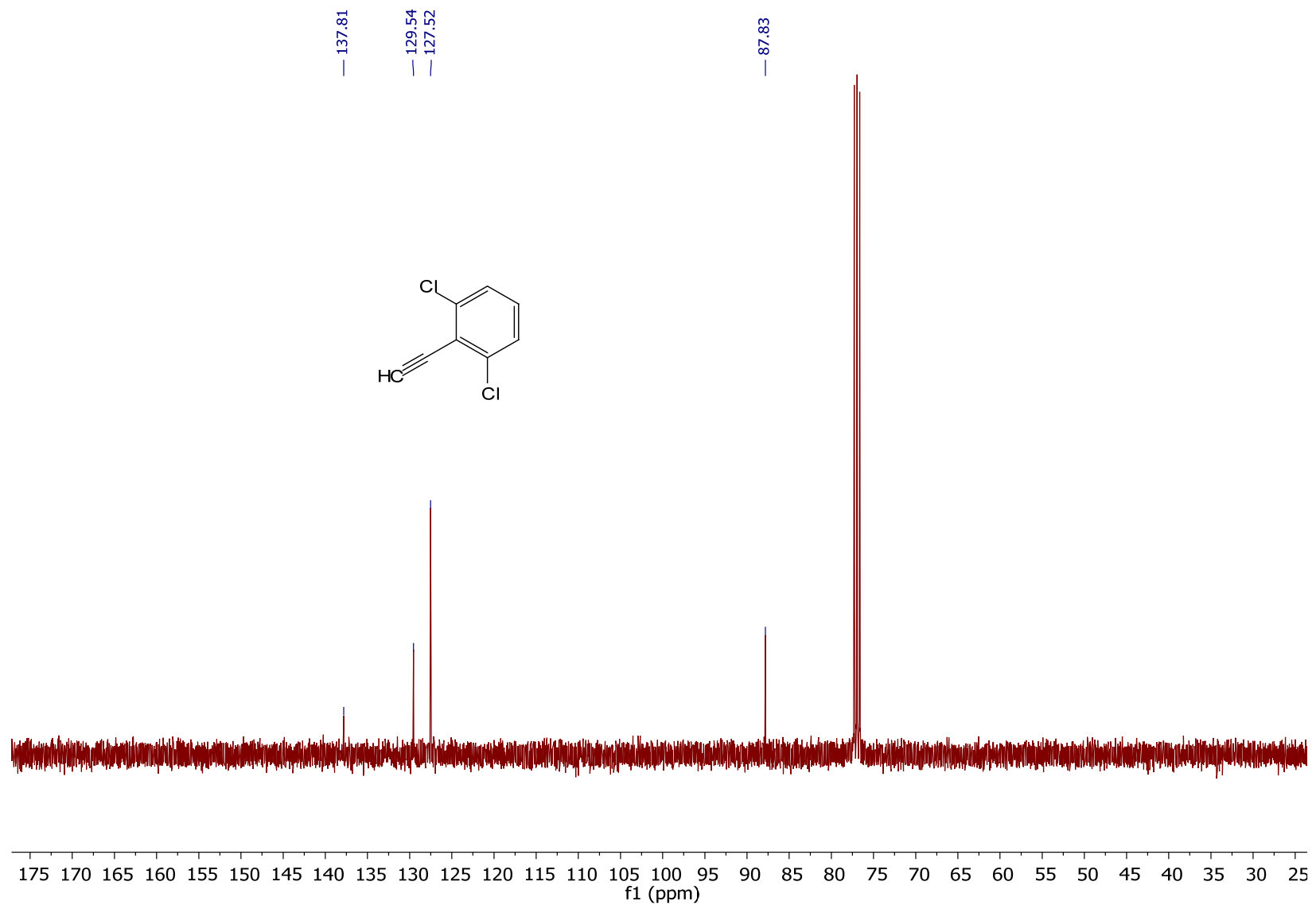


${ }^{1} \mathrm{H}$ NMR of ethynyl(methyl)diphenylsilane ( $\left.\mathrm{CDCl}_{3}, 400 \mathrm{MHz}\right)$

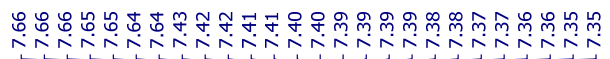

$$
\text { ํํำ }
$$
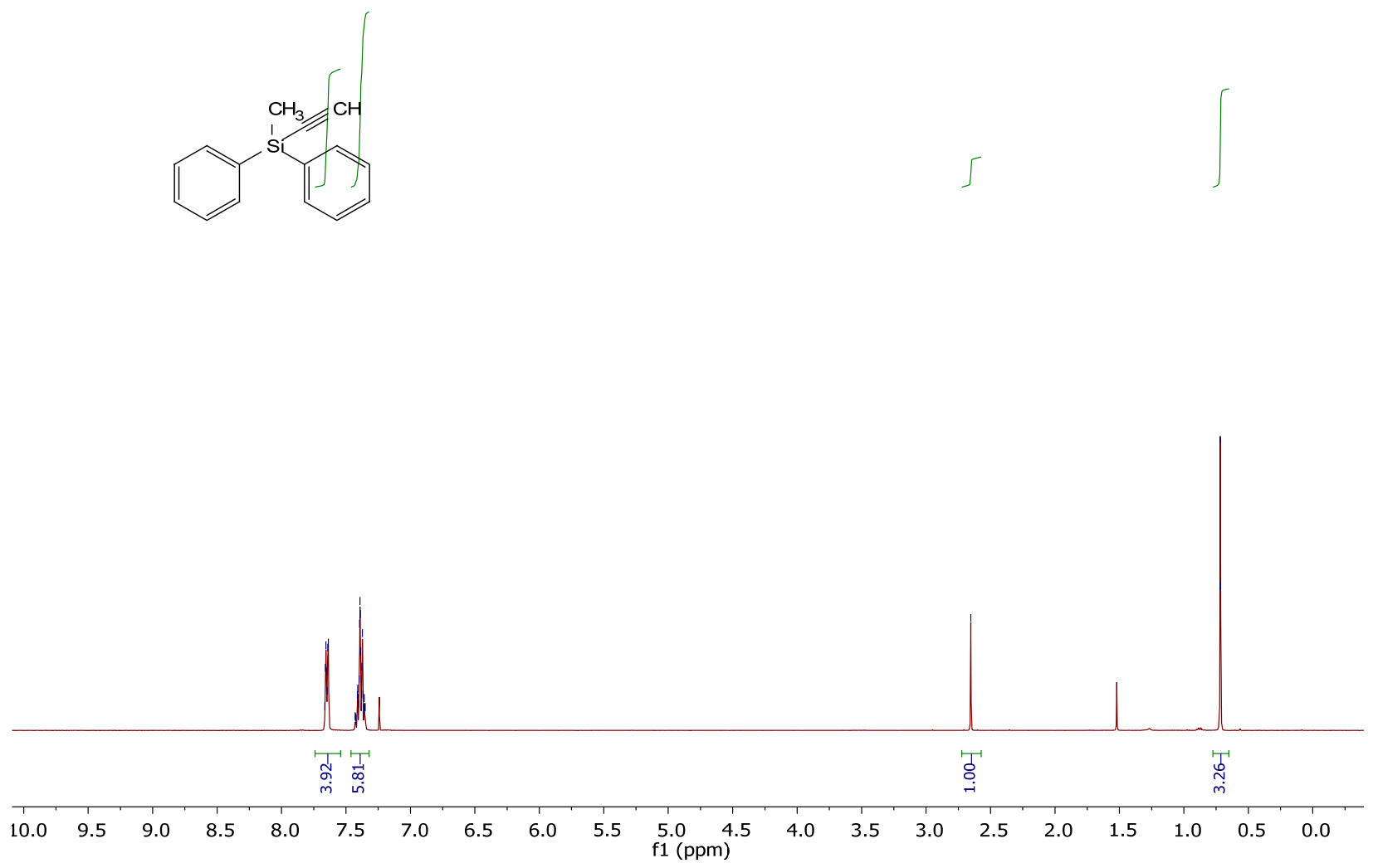


\section{${ }^{13} \mathrm{C}\left\{{ }^{1} \mathrm{H}\right\}$ NMR of ethynyl(methyl)diphenylsilane $\left(\mathrm{CDCl}_{3}, 100 \mathrm{MHz}\right)$}
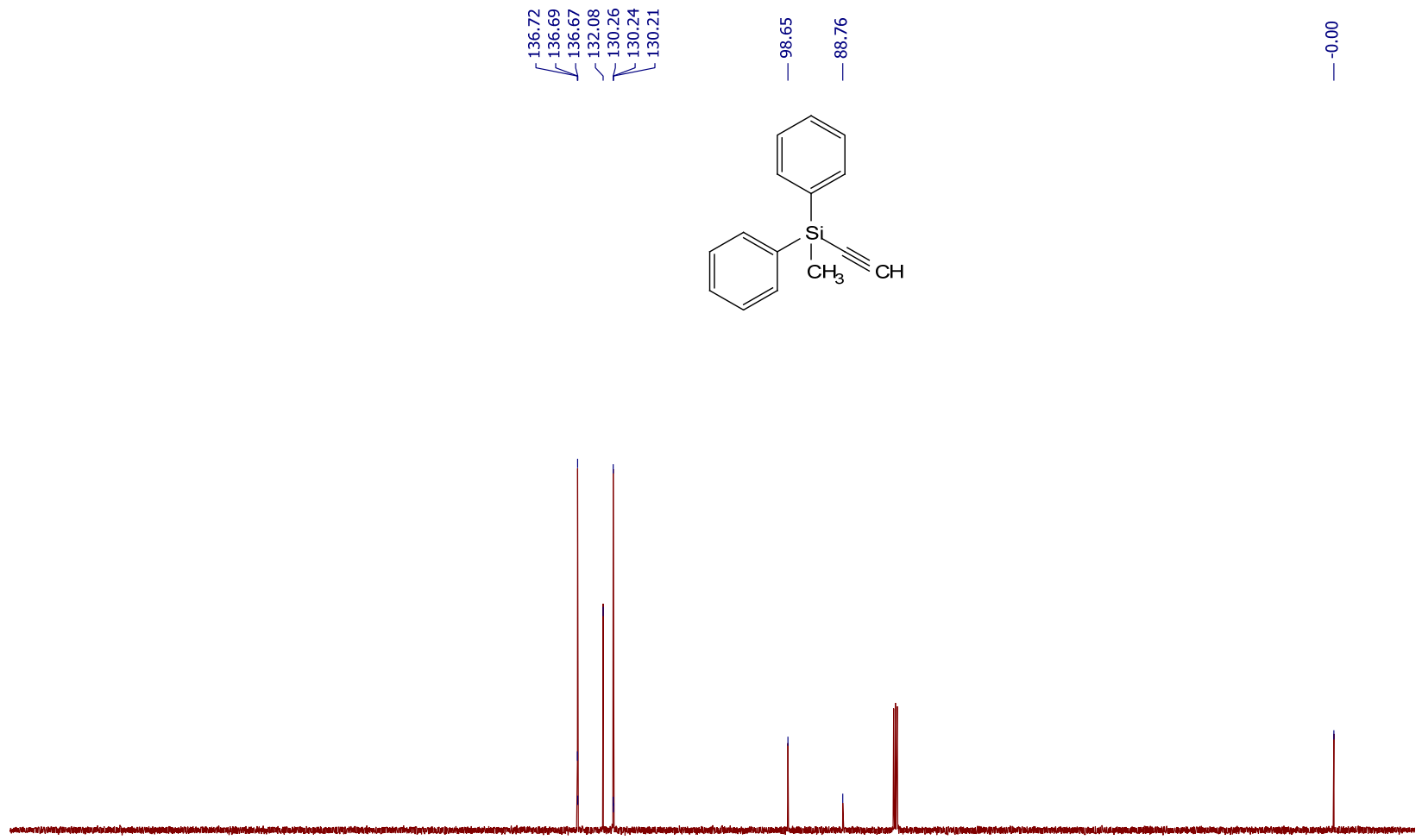

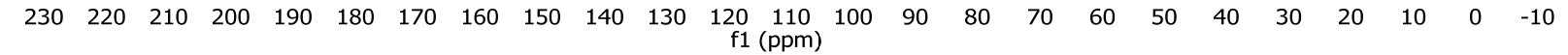


${ }^{1} \mathrm{H}$ NMR of ethynyldimethyl(phenyl)silane ( $\left.\mathrm{CDCl}_{3}, 400 \mathrm{MHz}\right)$

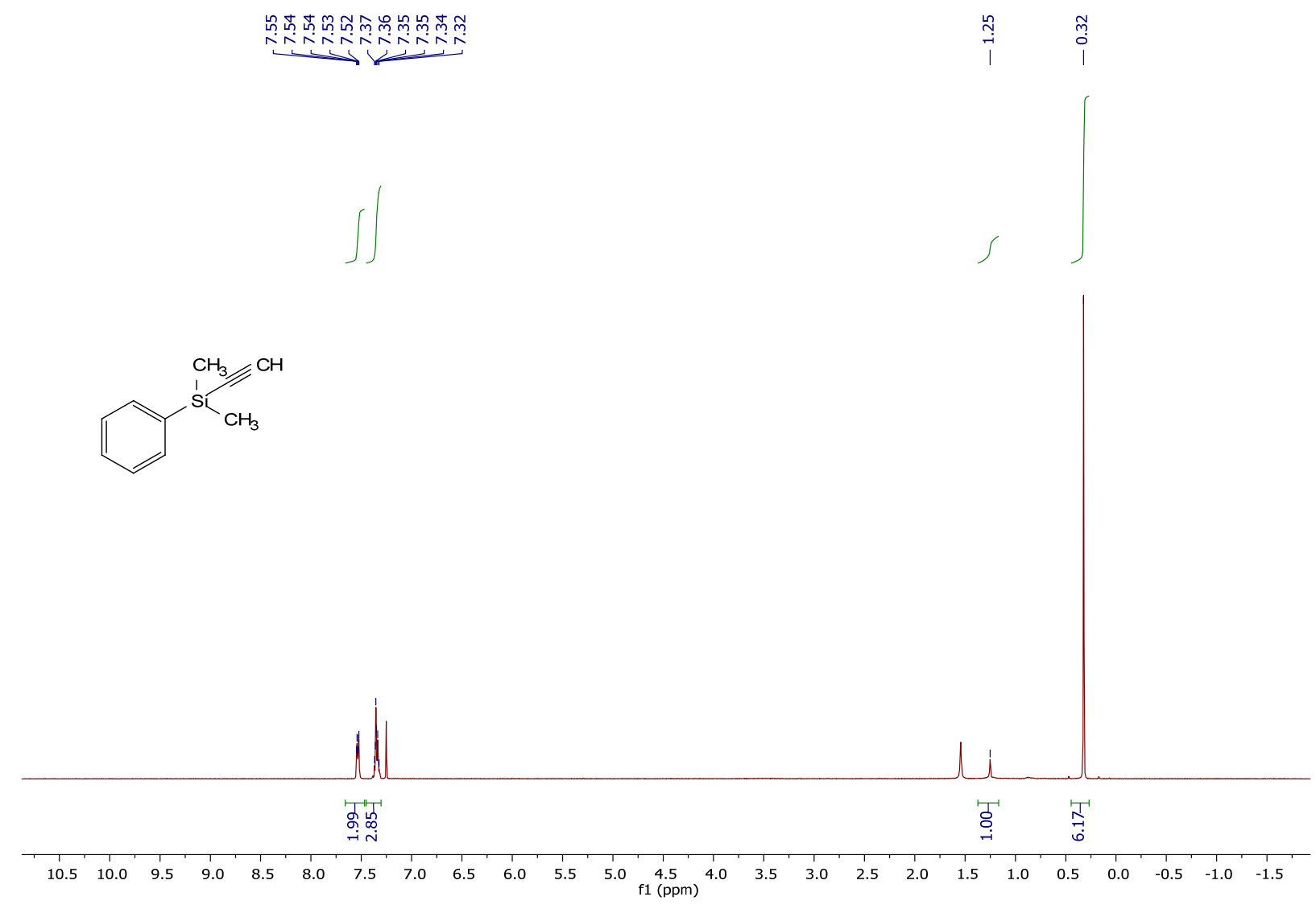


${ }^{13} \mathrm{C}\left\{{ }^{1} \mathrm{H}\right\}$ NMR of ethynyldimethyl(phenyl)silane $\left(\mathrm{CDCl}_{3}, 100 \mathrm{MHz}\right)$
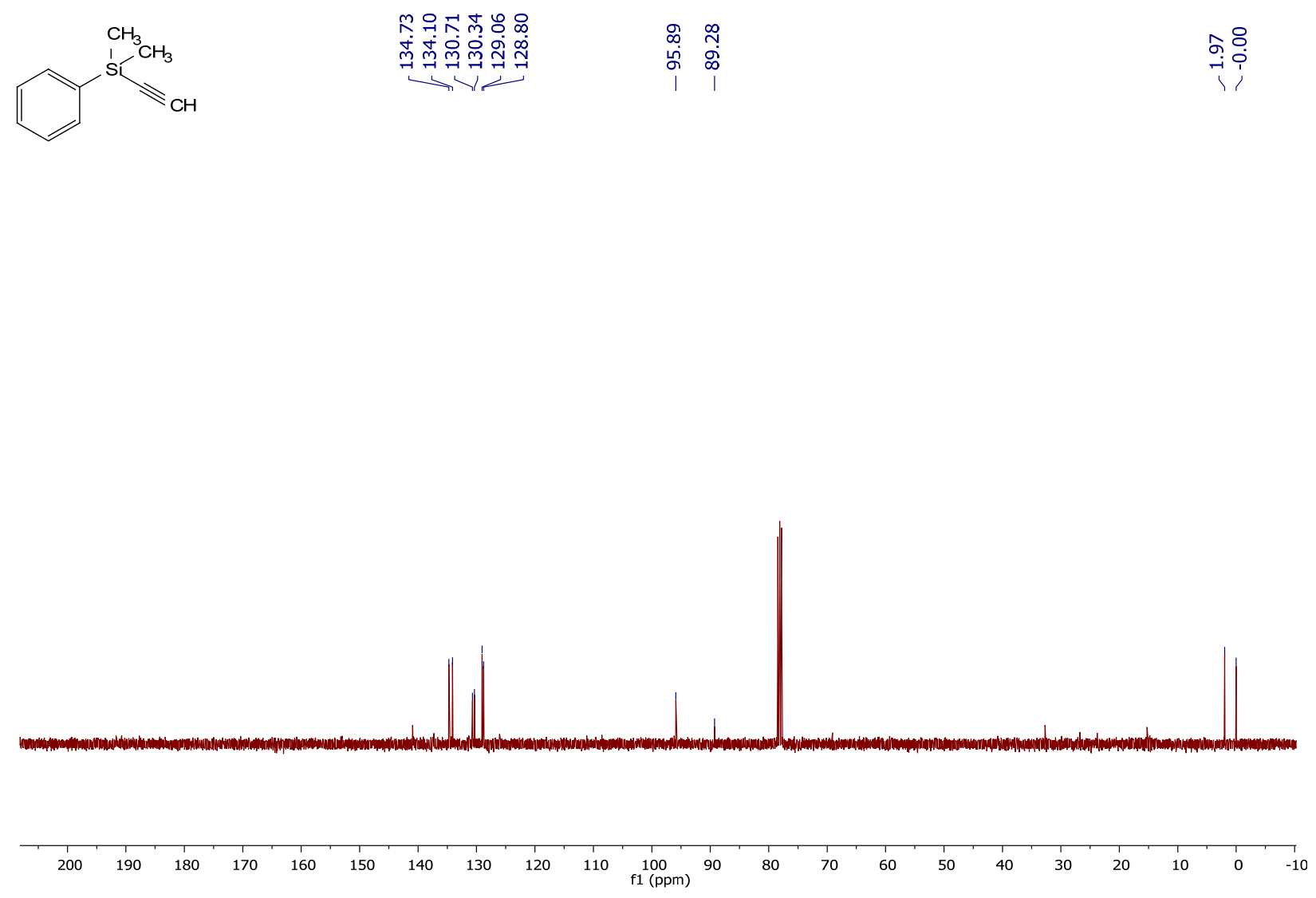


\section{${ }^{1} \mathrm{H}$ NMR of 12a $\left(\mathrm{CDCl}_{3}, 400 \mathrm{MHz}\right)$}

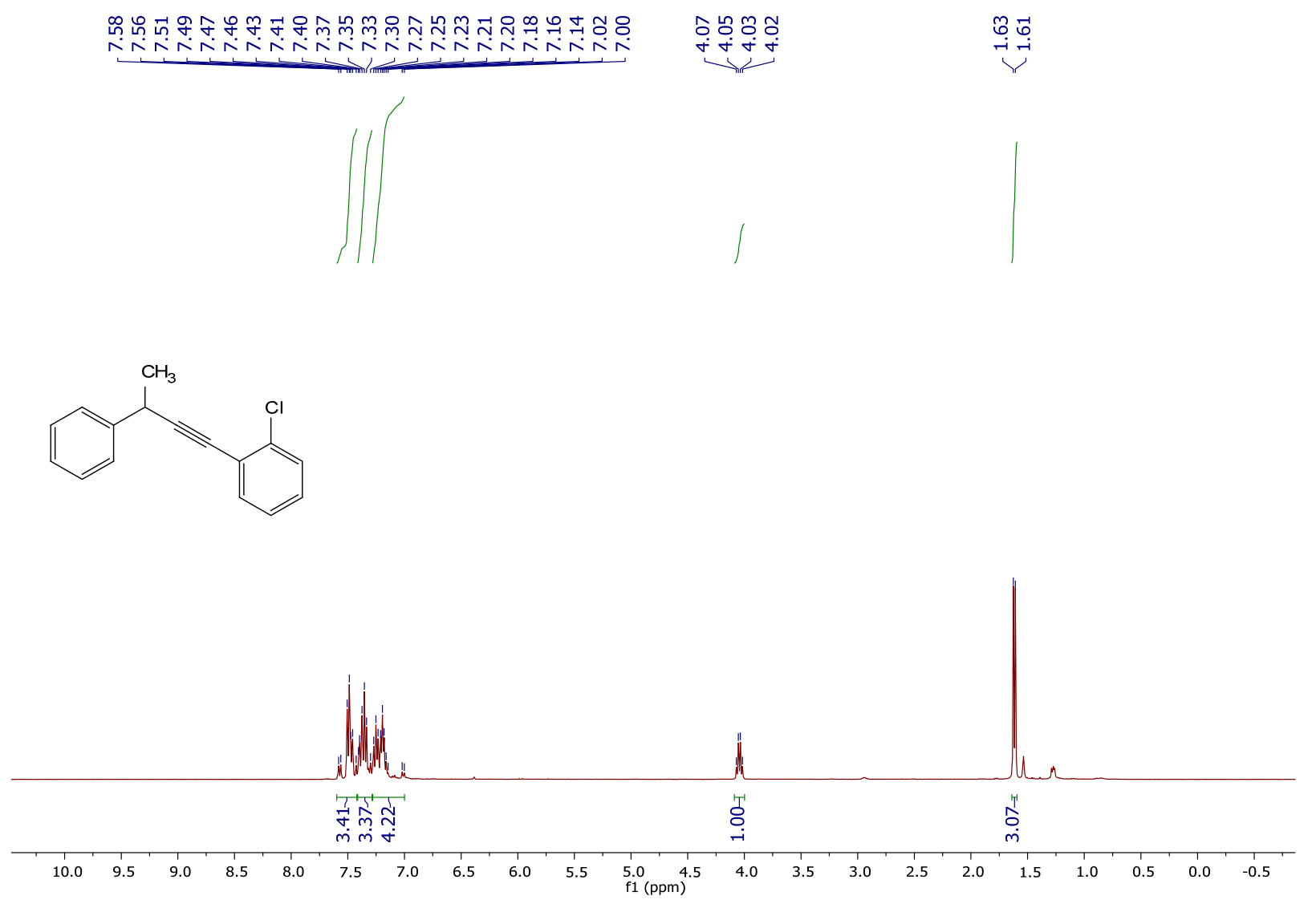




\section{${ }^{13} \mathrm{C}\left\{{ }^{1} \mathrm{H}\right\}$ NMR of 12a $\left(\mathrm{CDCl}_{3}, 100 \mathrm{MHz}\right)$}

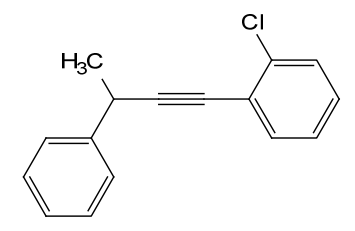

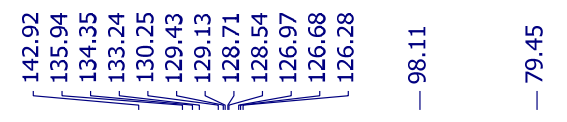

$\begin{array}{cc}-1 & 0 \\ & \stackrel{+}{~} \\ 1 & 1\end{array}$

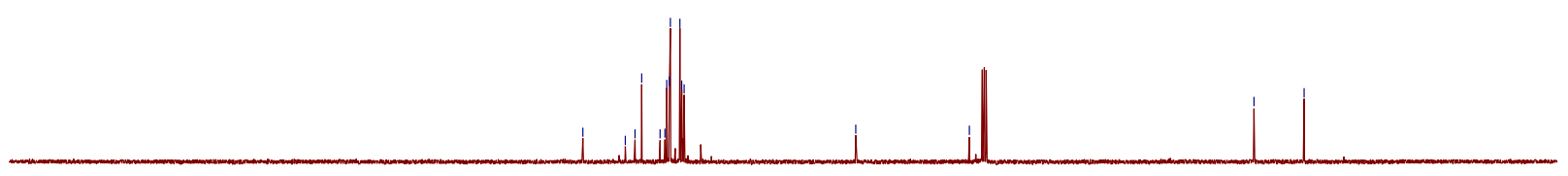

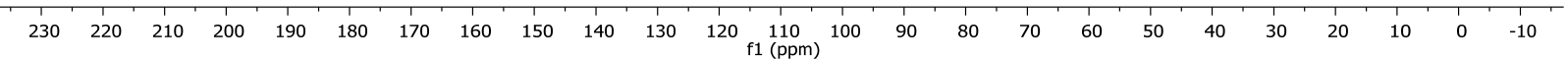


${ }^{1} \mathrm{H}$ NMR of $12 \mathrm{~b}\left(\mathrm{CDCl}_{3}, 400 \mathrm{MHz}\right)$

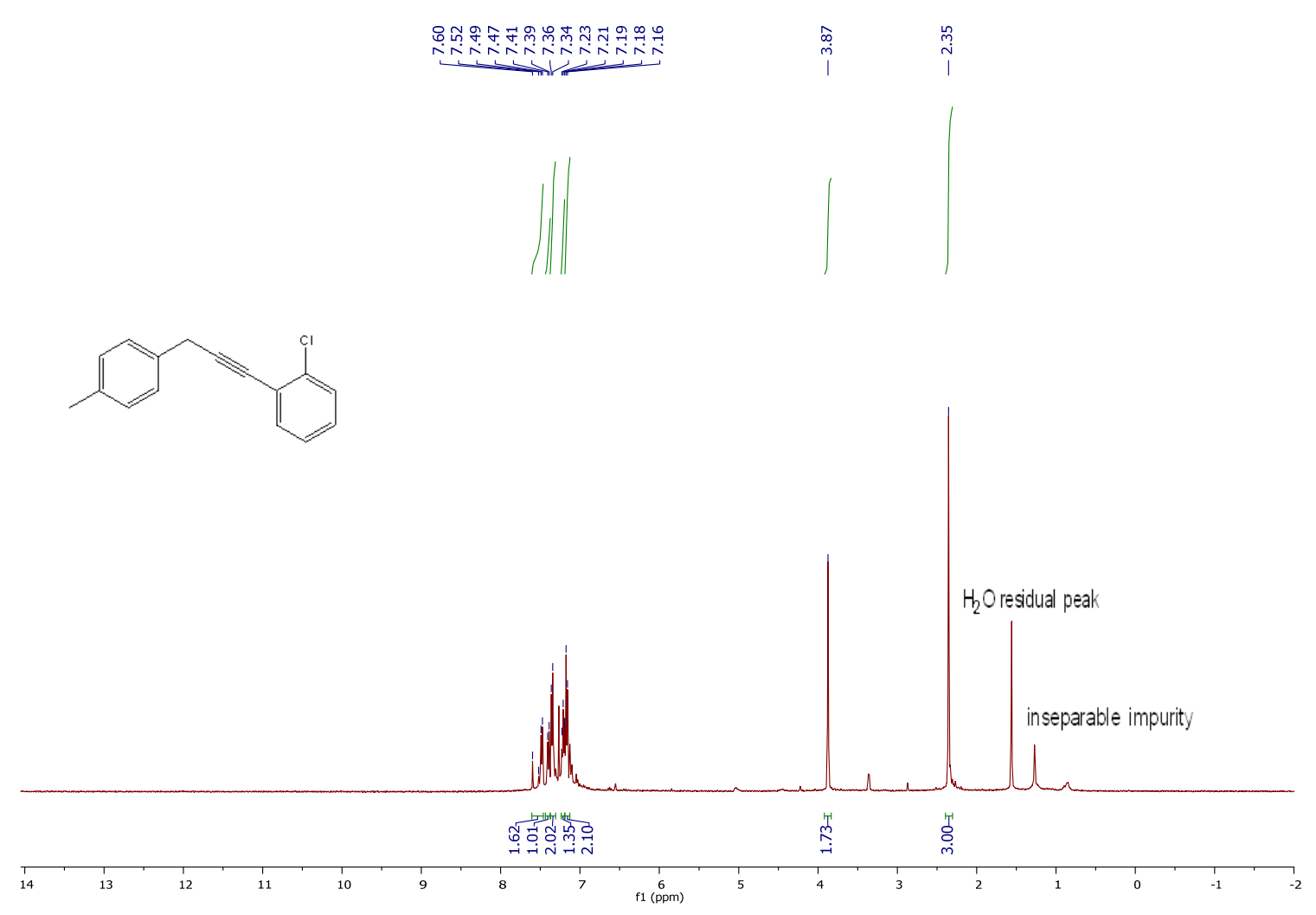


${ }^{13} \mathrm{C}\left\{{ }^{1} \mathrm{H}\right\}$ NMR of $12 \mathrm{~b}\left(\mathrm{CDCl}_{3}, 100 \mathrm{MHz}\right)$
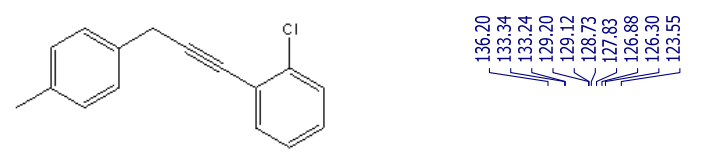

ભn

总

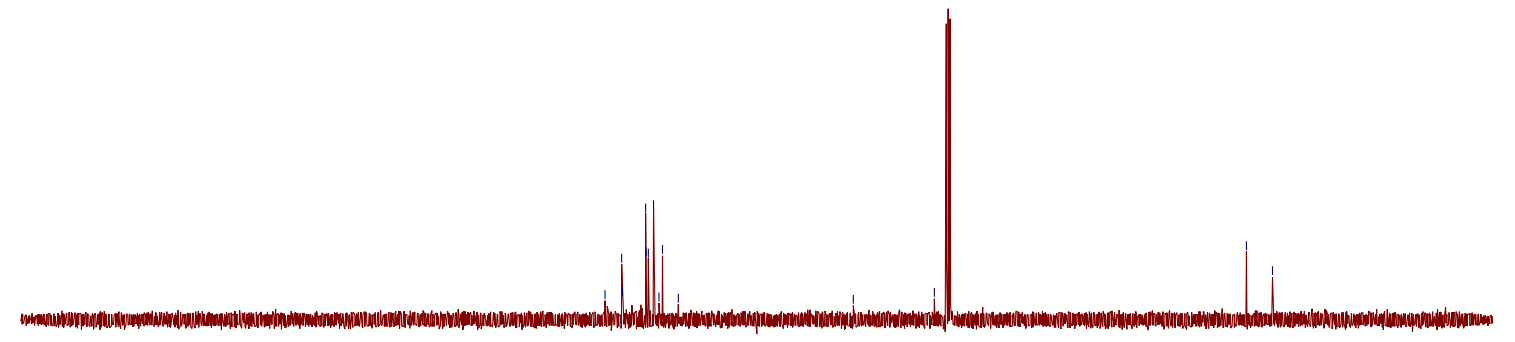

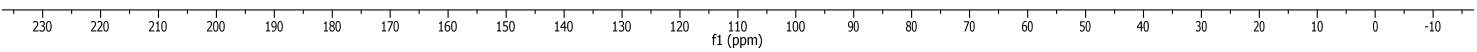


${ }^{1} \mathrm{H}$ NMR of $12 \mathrm{c}\left(\mathrm{CDCl}_{3}, 400 \mathrm{MHz}\right)$
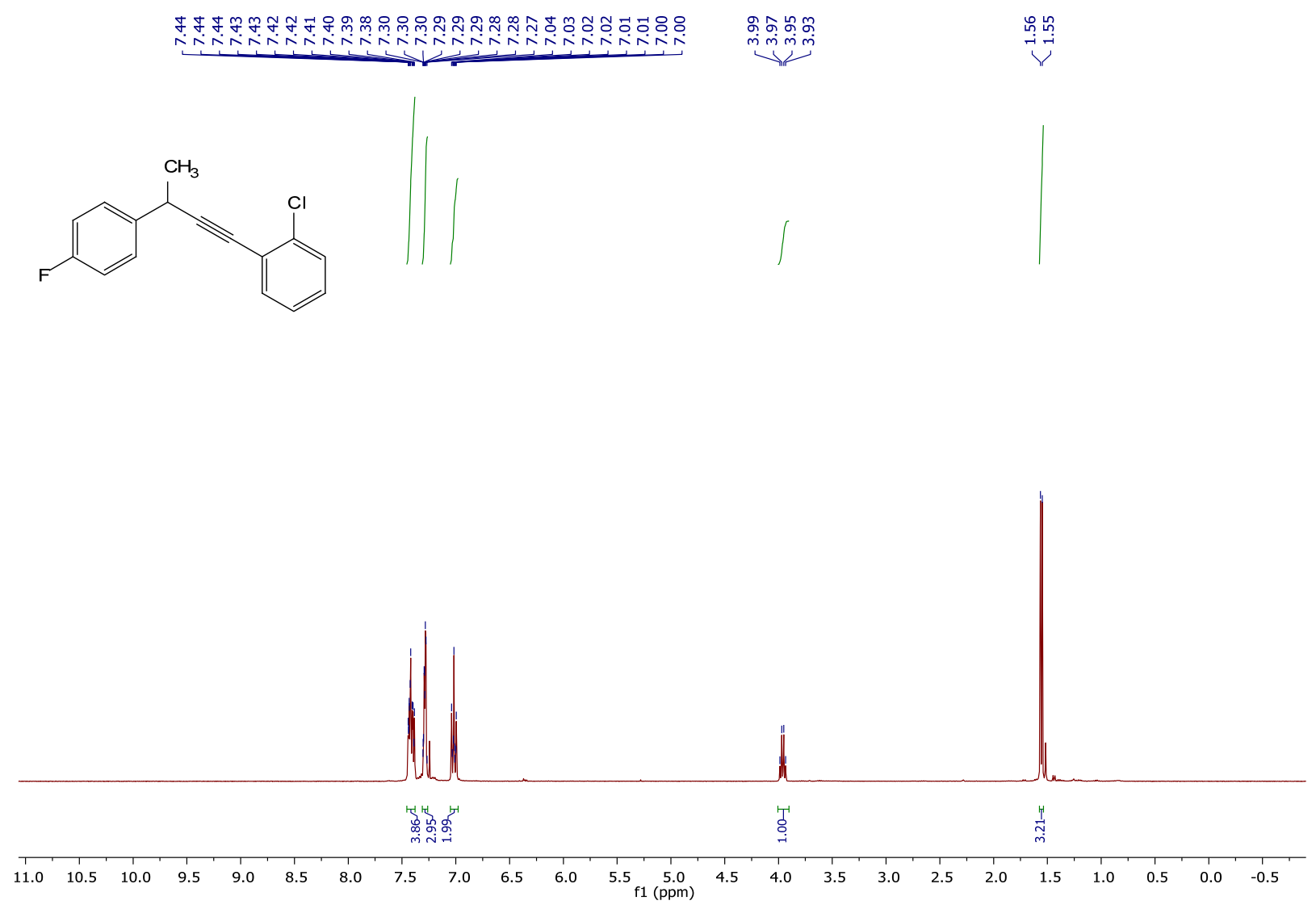


\section{${ }^{13} \mathrm{C}\left\{{ }^{1} \mathrm{H}\right\}$ NMR of $12 \mathrm{c}\left(\mathrm{CDCl}_{3}, 100 \mathrm{MHz}\right)$}

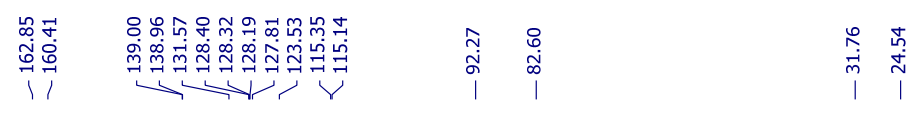<smiles>CC(C#Cc1ccccc1Cl)c1ccc(F)cc1</smiles>

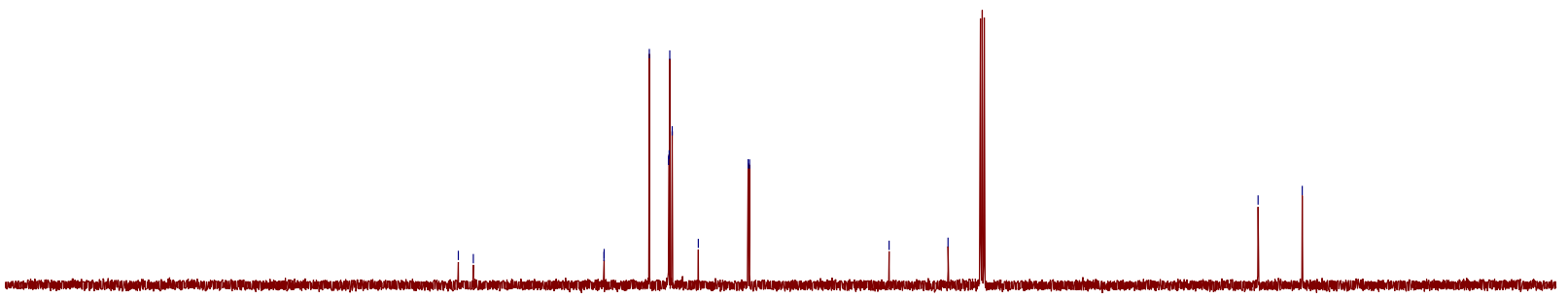

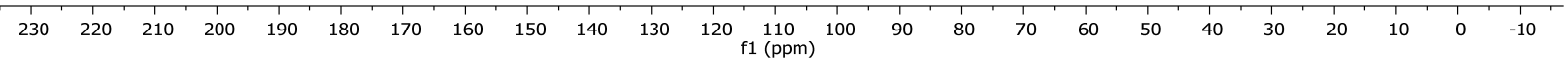


${ }^{19}$ F NMR of $12 \mathrm{c}\left(\mathrm{CDCl}_{3}, 387 \mathrm{MHz}\right)$<smiles>CC(C#Cc1ccccc1O)c1ccc(F)cc1</smiles>

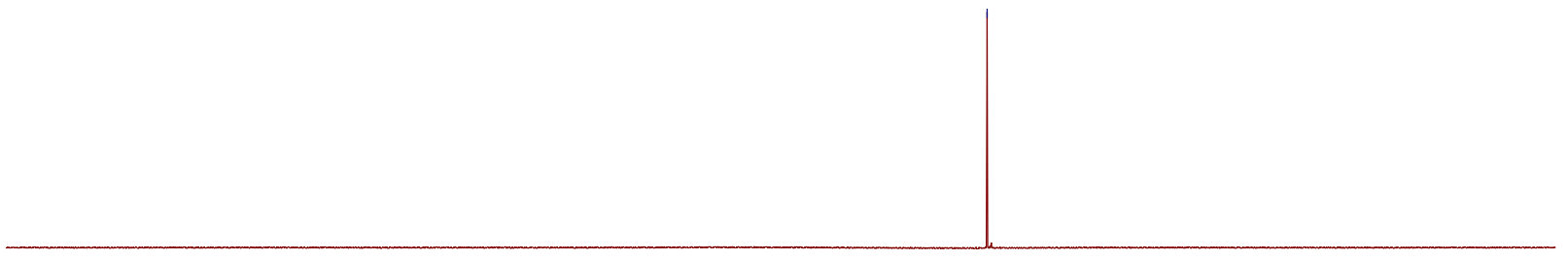

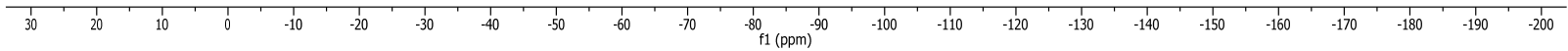


${ }^{1} \mathrm{H}$ NMR of $12 \mathrm{~d}\left(\mathrm{CDCl}_{3}, 400 \mathrm{MHz}\right)$

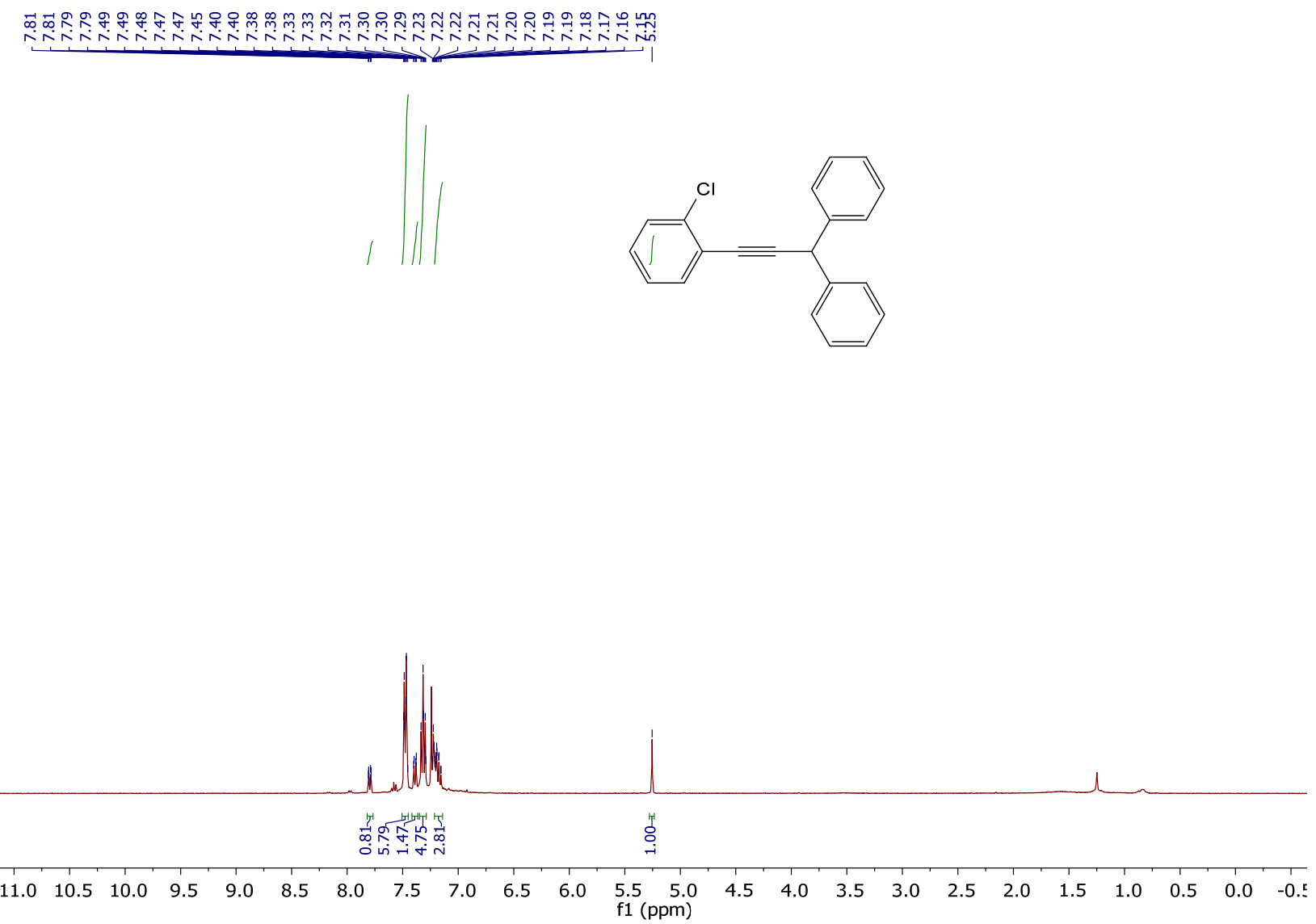


${ }^{13} \mathrm{C}\left\{{ }^{1} \mathrm{H}\right\}$ NMR of $12 \mathrm{~d}\left(\mathrm{CDCl}_{3}, 100 \mathrm{MHz}\right)$
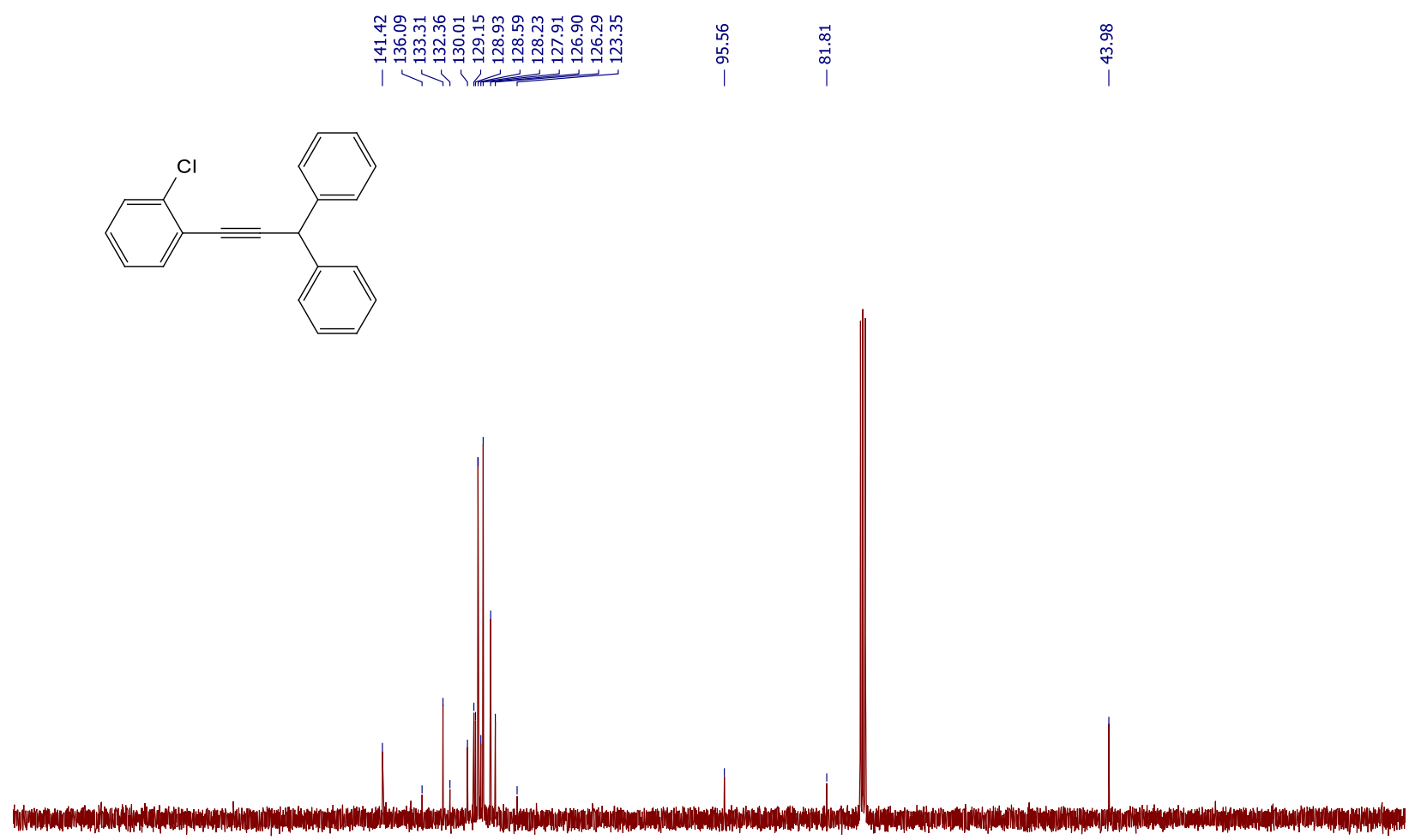

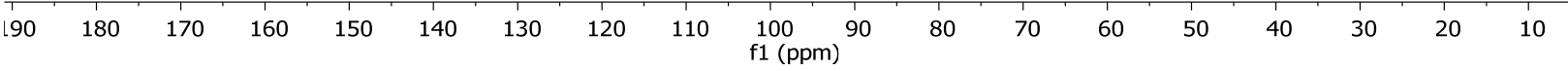


${ }^{1} \mathrm{H}$ NMR of $12 \mathrm{e}\left(\mathrm{CDCl}_{3}, 400 \mathrm{MHz}\right)$

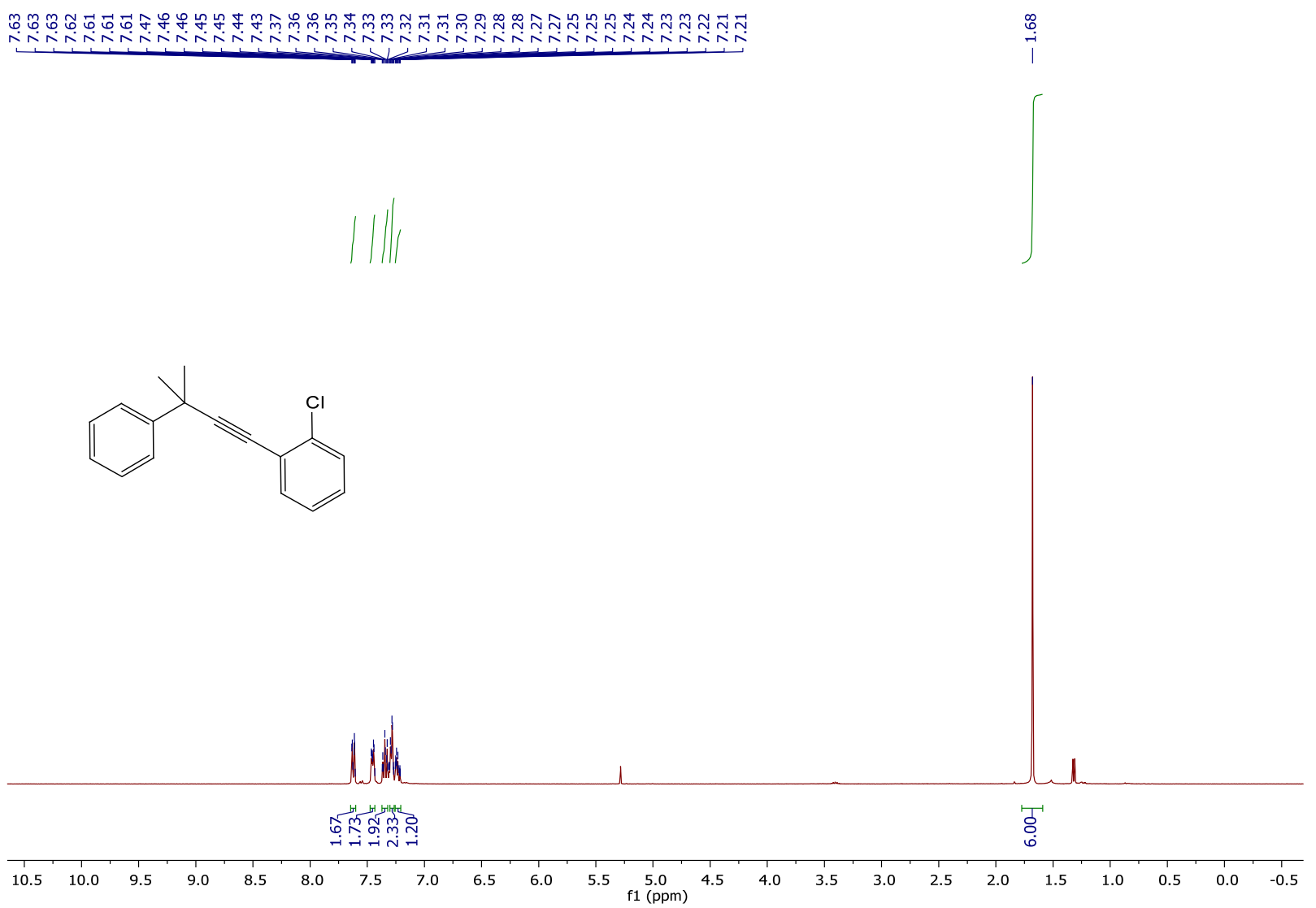




\section{${ }^{13} \mathrm{C}\left\{{ }^{1} \mathrm{H}\right\}$ NMR of $12 \mathrm{e}\left(\mathrm{CDCl}_{3}, 100 \mathrm{MHz}\right)$}

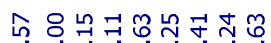

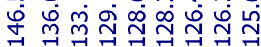<smiles>CC(C)(C#Cc1ccccc1Cl)c1ccccc1</smiles>

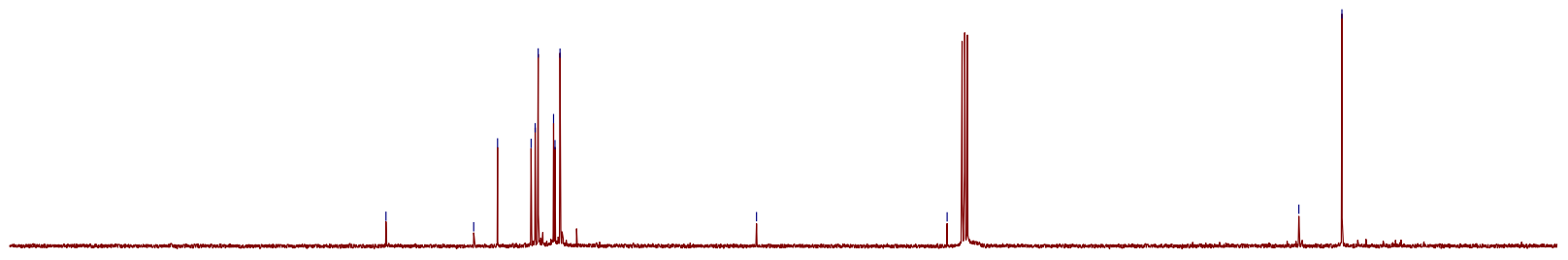

$190 \quad 180 \quad 170$
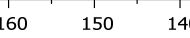


\section{${ }^{1} \mathrm{H}$ NMR of $12 \mathrm{f}\left(\mathrm{CDCl}_{3}, 400 \mathrm{MHz}\right)$}

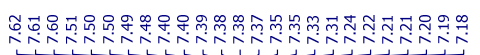
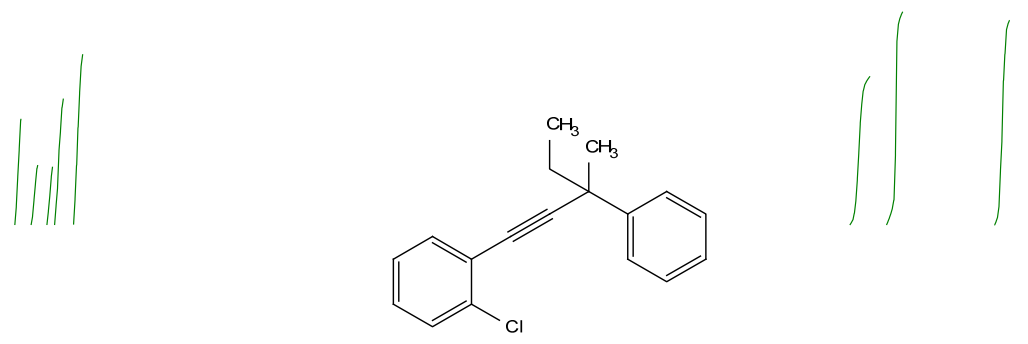

ن|

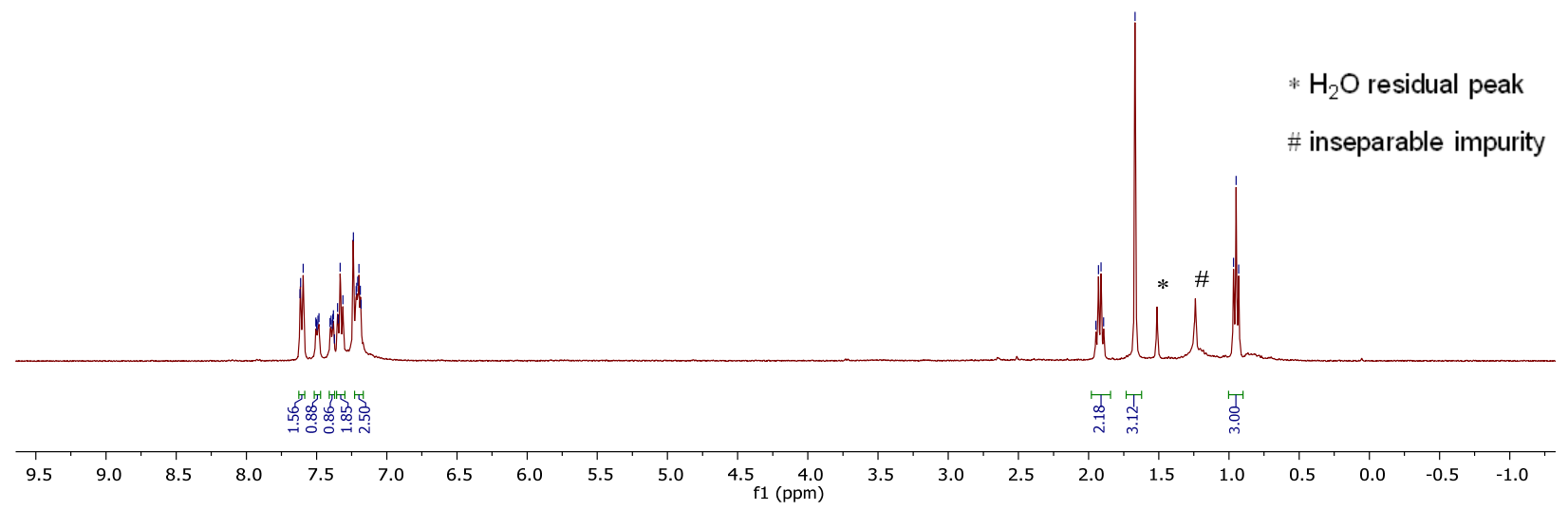


${ }^{13} \mathrm{C}\left\{{ }^{1} \mathrm{H}\right\}$ NMR of $12 \mathrm{f}\left(\mathrm{CDCl}_{3}, 100 \mathrm{MHz}\right)$

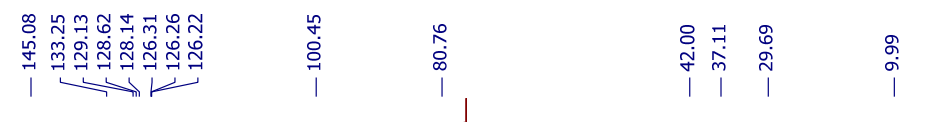<smiles>CCC(C)(C#Cc1ccccc1Cl)c1ccccc1</smiles>

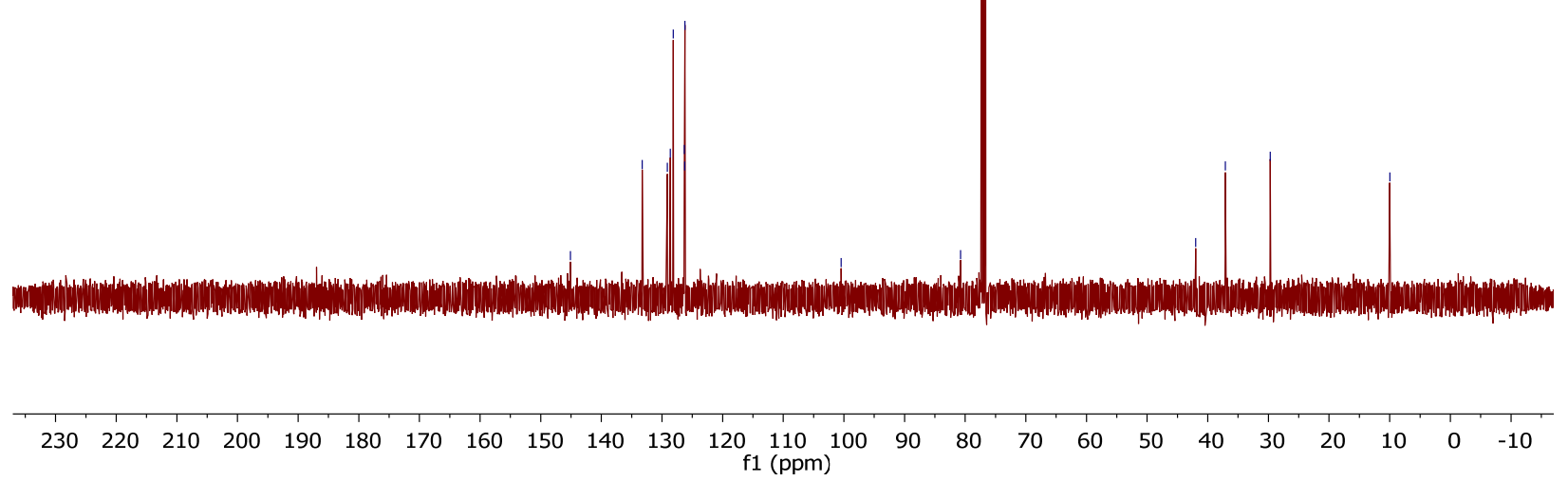


${ }^{1} \mathrm{H}$ NMR of $12 \mathrm{~g}\left(\mathrm{CDCl}_{3}, 400 \mathrm{MHz}\right)$

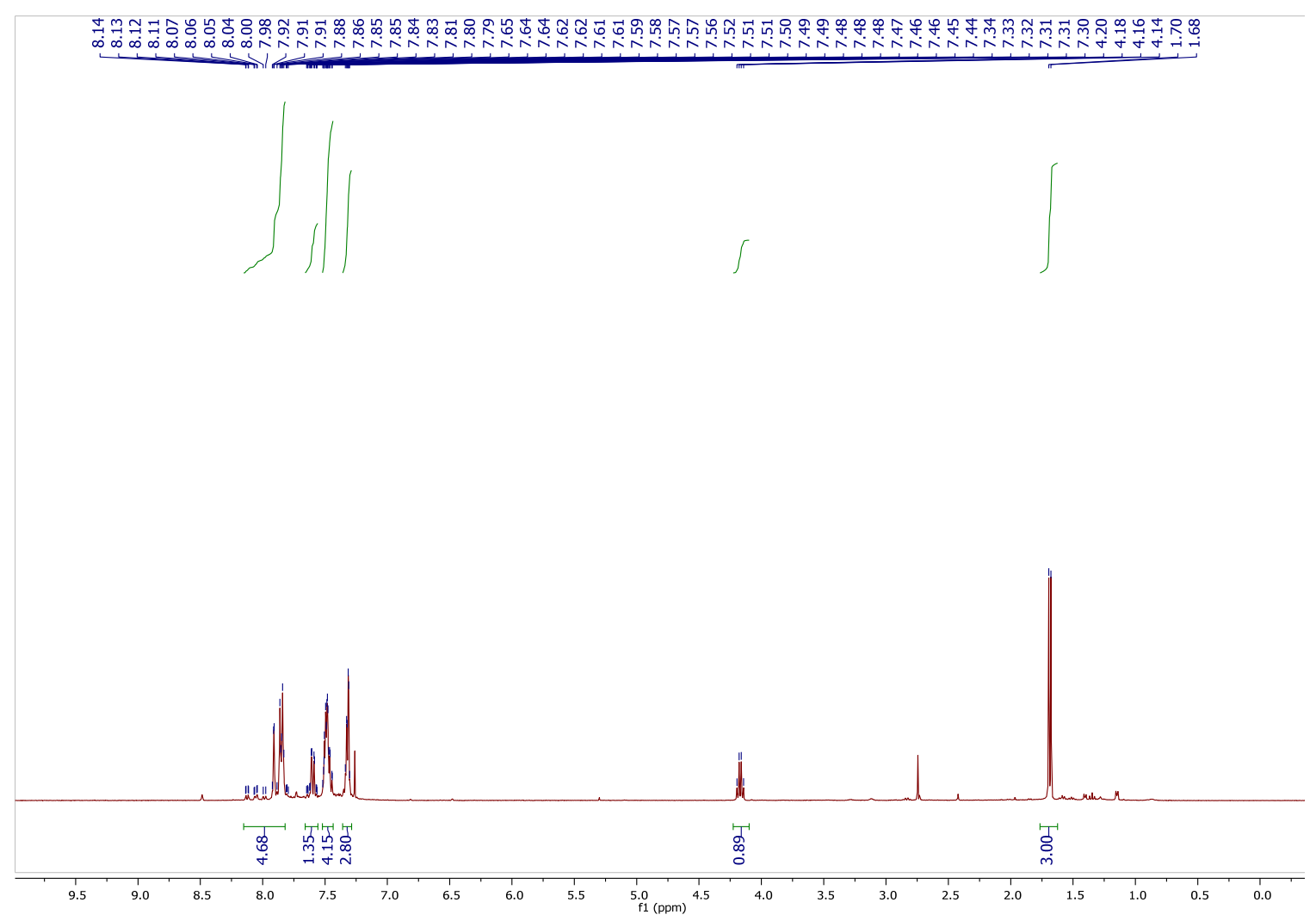




\section{${ }^{13} \mathrm{C}\left\{{ }^{1} \mathrm{H}\right\}$ NMR of $12 \mathrm{~g}\left(\mathrm{CDCl}_{3}, 100 \mathrm{MHz}\right)$}

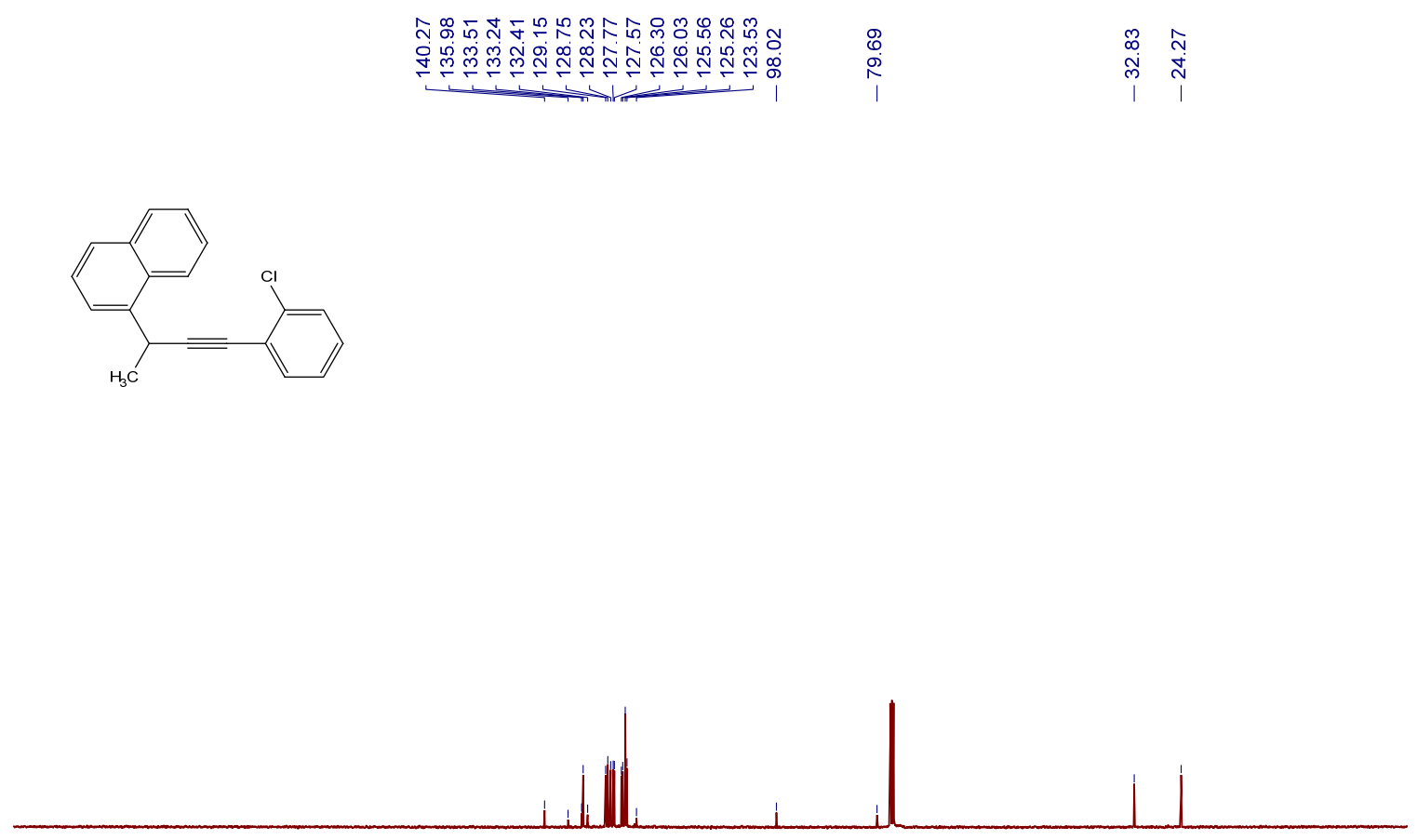

$\begin{array}{lllllllllllllllllllllllll}230 & 220 & 210 & 200 & 190 & 180 & 170 & 160 & 150 & 140 & 130 & 120 & 110 & 100 & 90 & 80 & 70 & 60 & 50 & 40 & 30 & 20 & 10 & 0 & -10\end{array}$ $\mathrm{f} 1(\mathrm{ppm})$ 
${ }^{1} \mathrm{H}$ NMR of $12 \mathrm{~h}\left(\mathrm{CDCl}_{3}, 400 \mathrm{MHz}\right)$

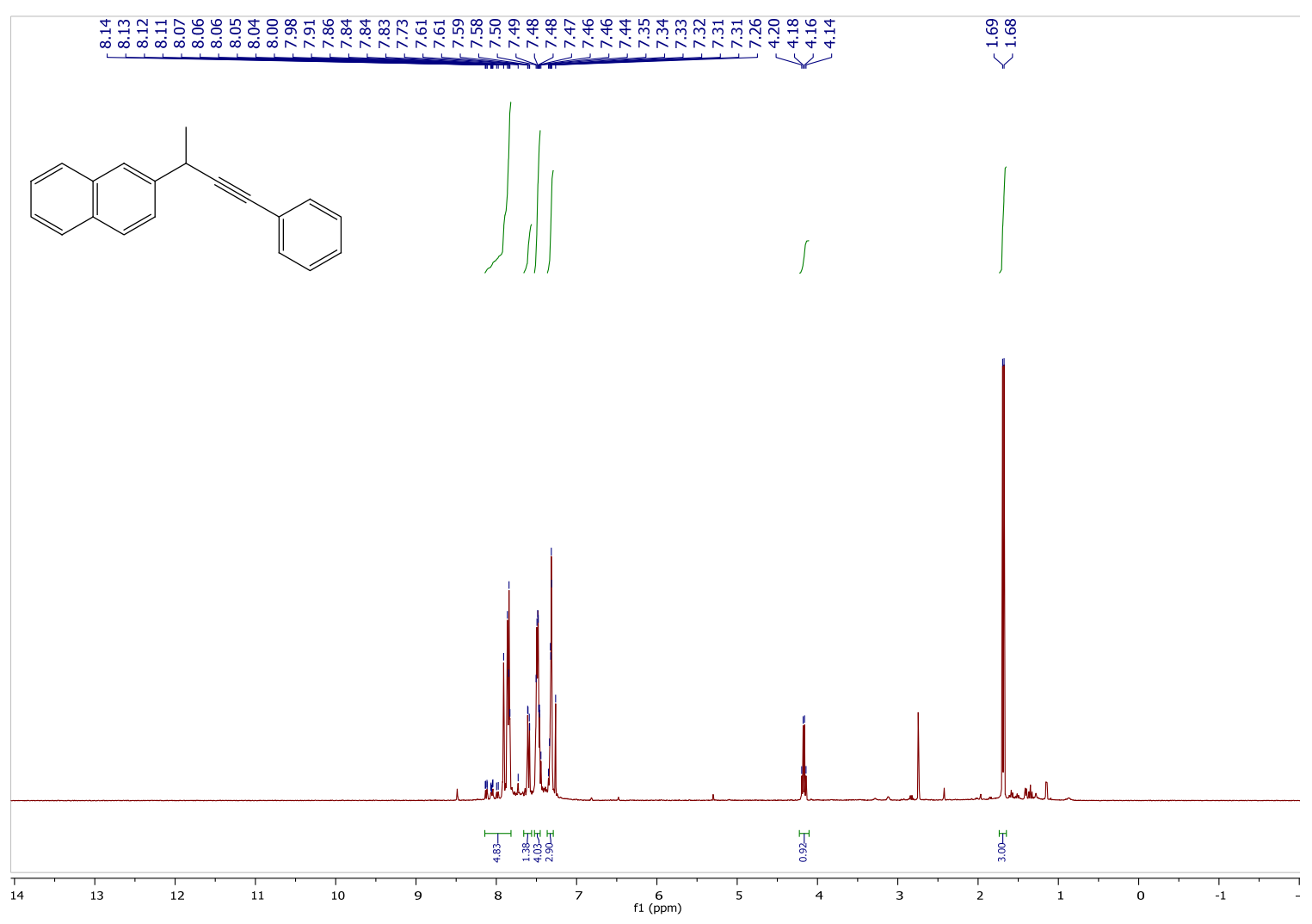


${ }^{13} \mathrm{C}\left\{{ }^{1} \mathrm{H}\right\}$ NMR of $12 \mathrm{~h}\left(\mathrm{CDCl}_{3}, 100 \mathrm{MHz}\right)$

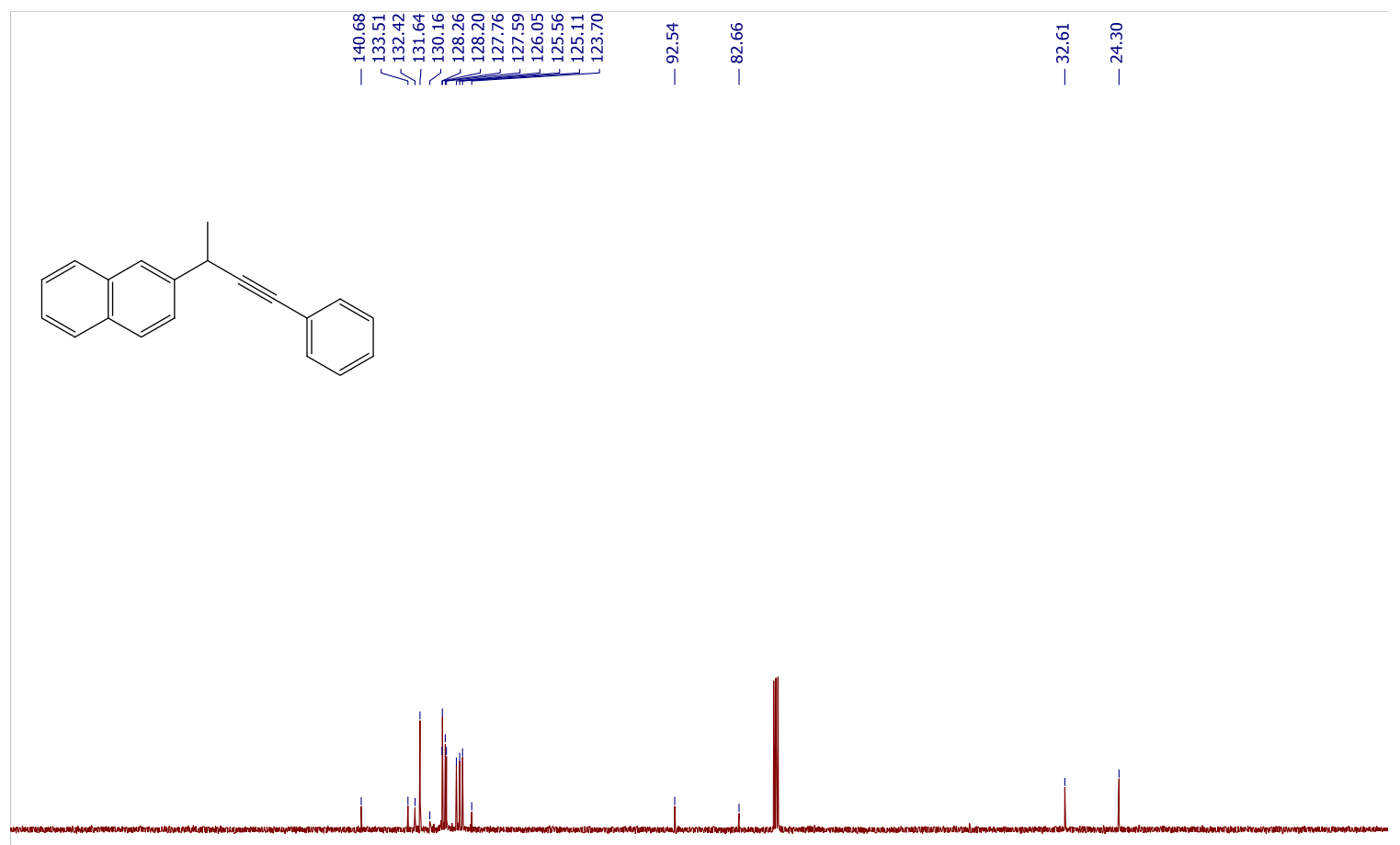

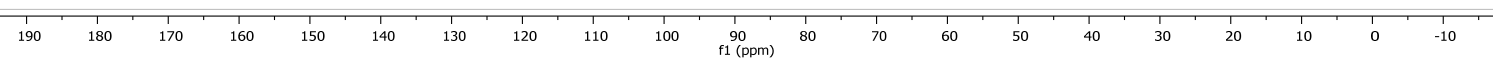


${ }^{1} \mathrm{H}$ NMR of 13a $\left(\mathrm{CDCl}_{3}, 400 \mathrm{MHz}\right)$
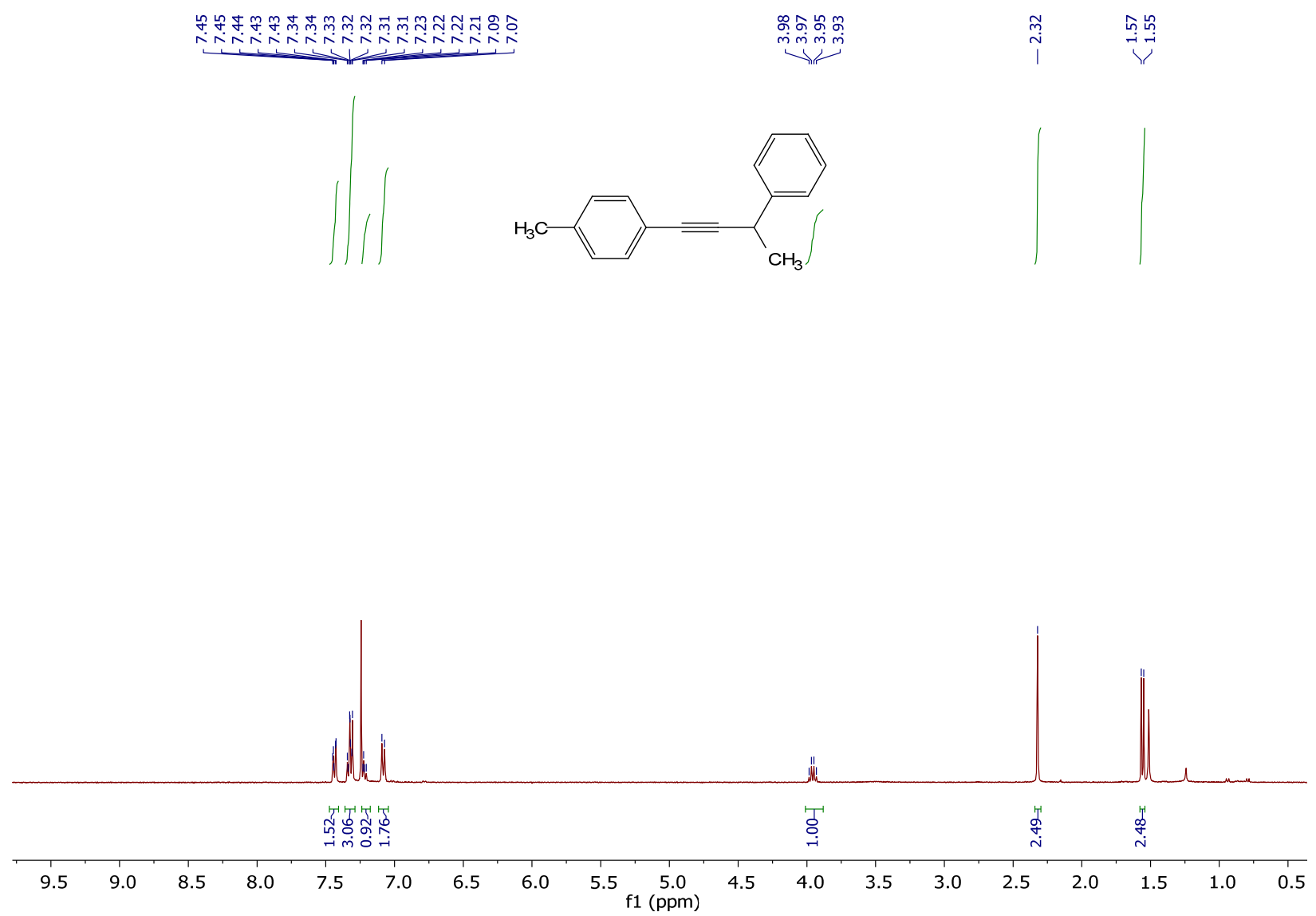
${ }^{13} \mathrm{C}\left\{{ }^{1} \mathrm{H}\right\}$ NMR of 13a $\left(\mathrm{CDCl}_{3}, 100 \mathrm{MHz}\right)$
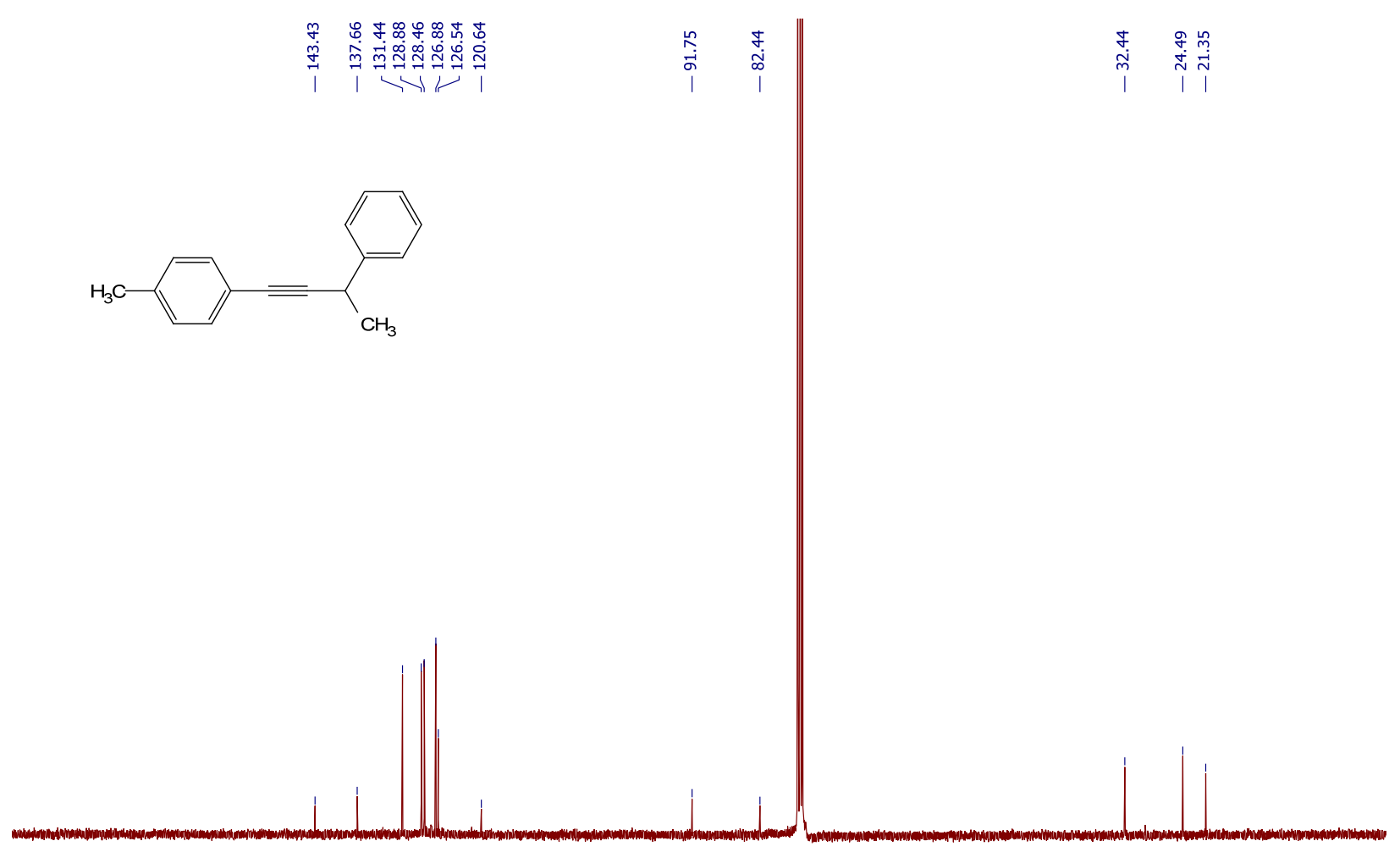

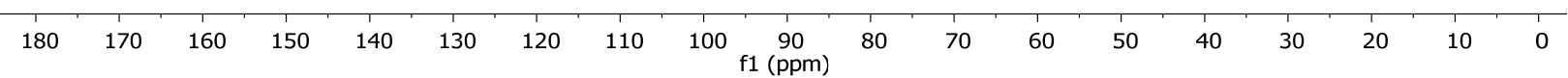


${ }^{1} \mathrm{H}$ NMR of $13 \mathrm{~b}\left(\mathrm{CDCl}_{3}, 400 \mathrm{MHz}\right)$

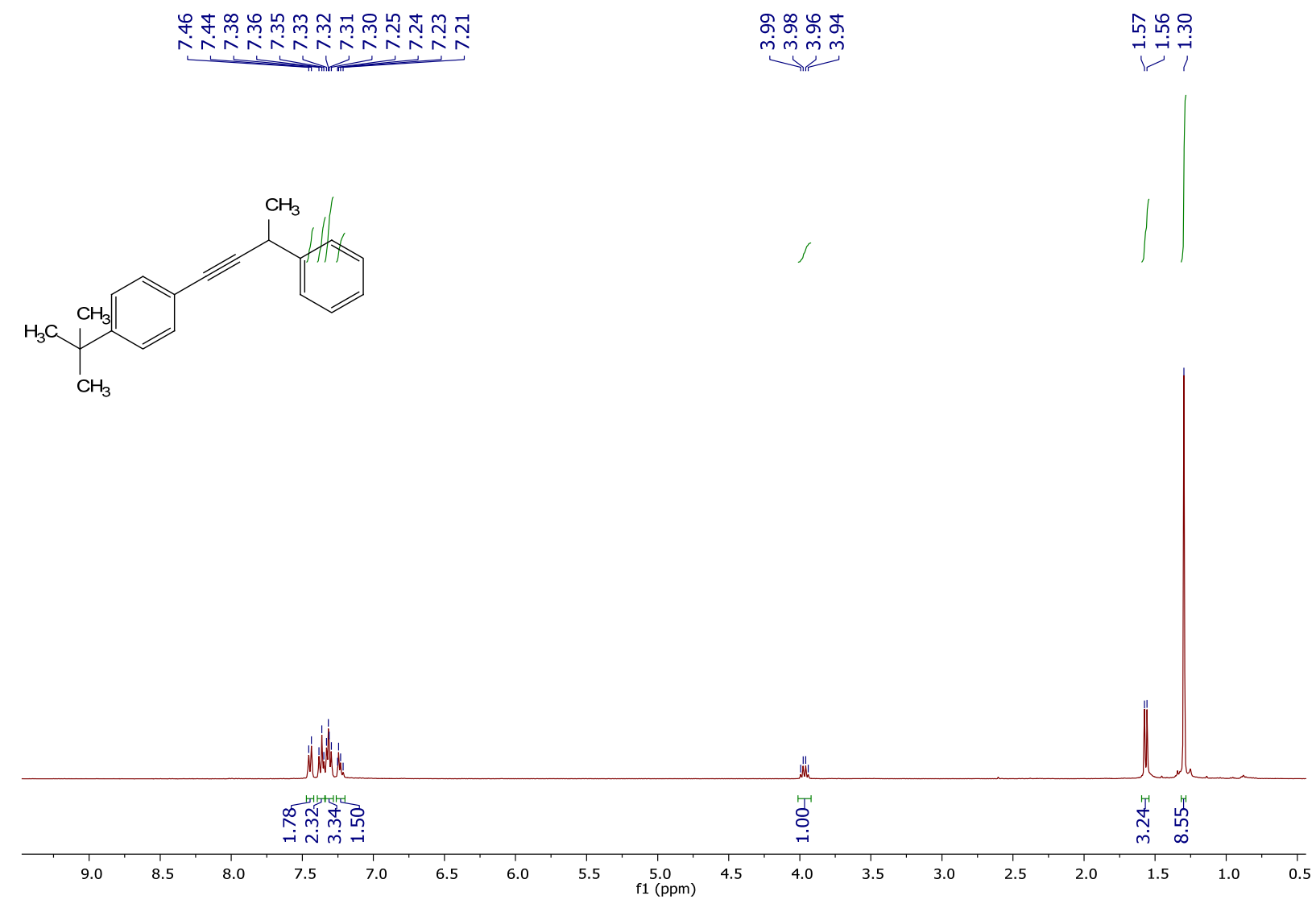


${ }^{13} \mathrm{C}\left\{{ }^{1} \mathrm{H}\right\}$ NMR of $13 \mathrm{~b}\left(\mathrm{CDCl}_{3}, 100 \mathrm{MHz}\right)$

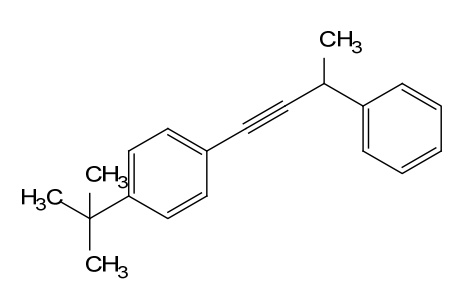

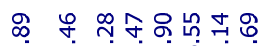

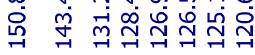

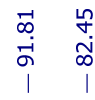

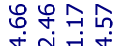

लिखल

11,

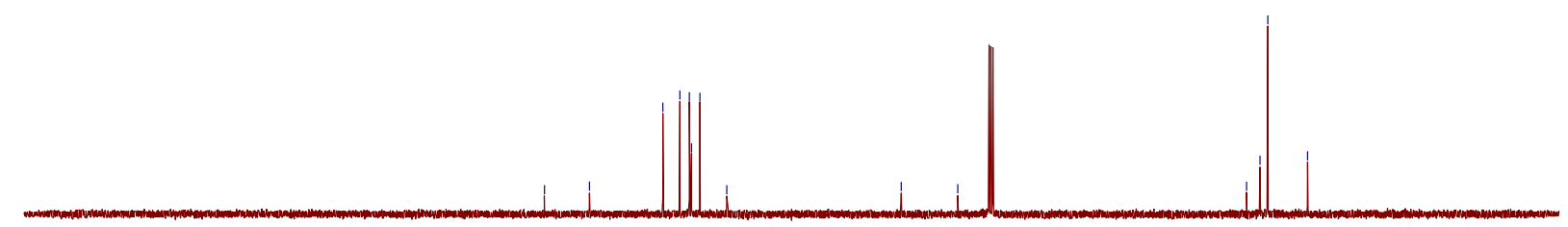

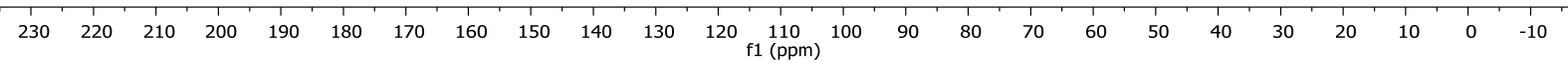


${ }^{1} \mathrm{H}$ NMR of $13 \mathrm{c}\left(\mathrm{CDCl}_{3}, 400 \mathrm{MHz}\right)$

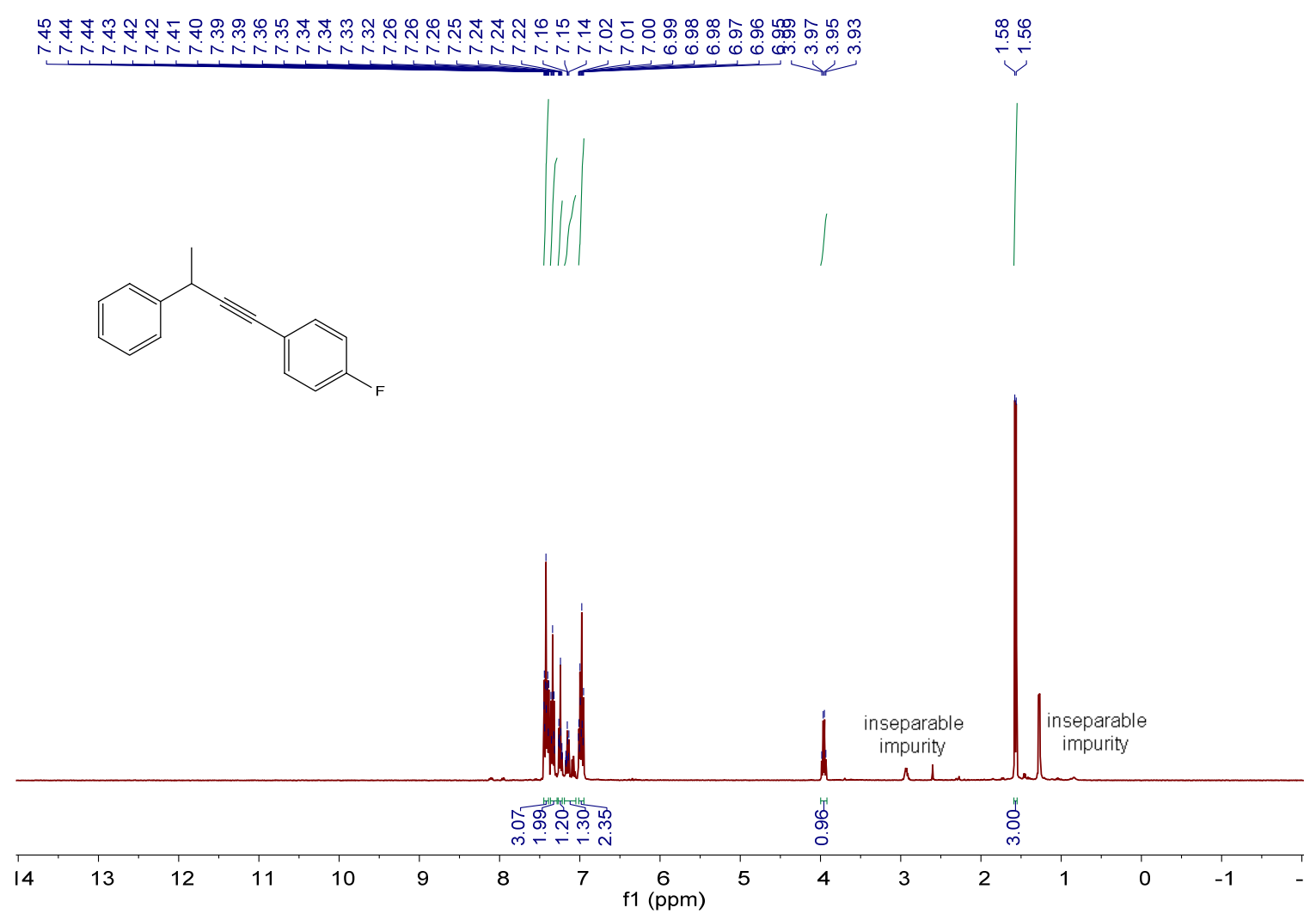


${ }^{13} \mathrm{C}\left\{{ }^{1} \mathrm{H}\right\}$ NMR of $13 \mathrm{c}\left(\mathrm{CDCl}_{3}, 100 \mathrm{MHz}\right)$
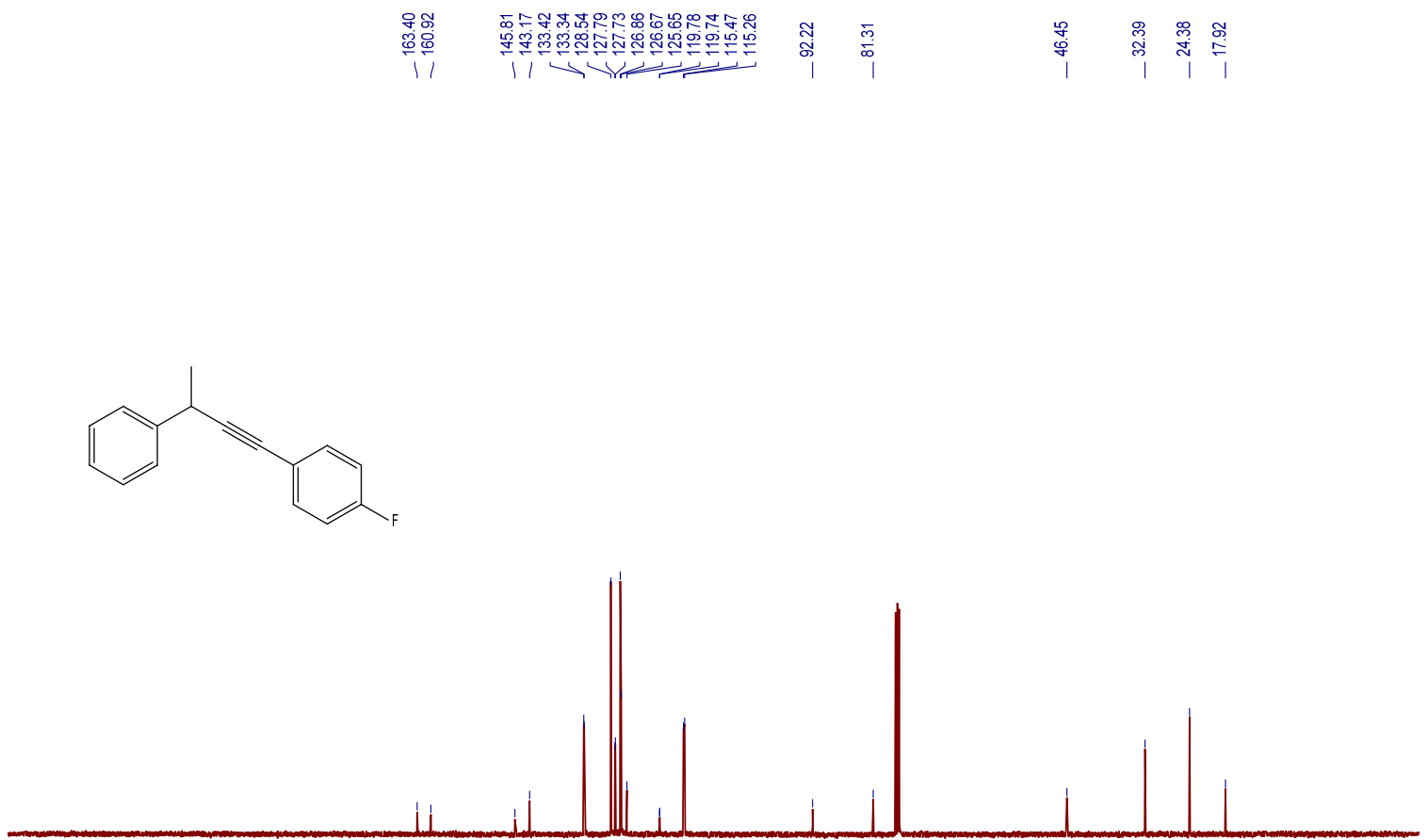

\begin{tabular}{llllllllllllllllllllllllll}
\hline & 230 & 220 & 210 & 200 & 190 & 180 & 170 & 160 & 150 & 140 & 130 & 120 & 110 & 100 & 90 & 80 & 70 & 60 & 50 & 40 & 30 & 20 & 10 & 0 & -10
\end{tabular} 
${ }^{19} \mathrm{~F}$ NMR of $13 \mathrm{c}\left(\mathrm{CDCl}_{3}, 387 \mathrm{MHz}\right)$

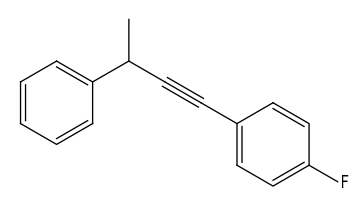


${ }^{1} \mathrm{H}$ NMR of $13 \mathrm{~d}\left(\mathrm{CDCl}_{3}, 400 \mathrm{MHz}\right)$

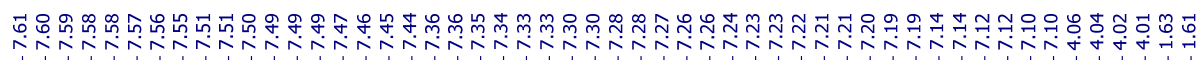
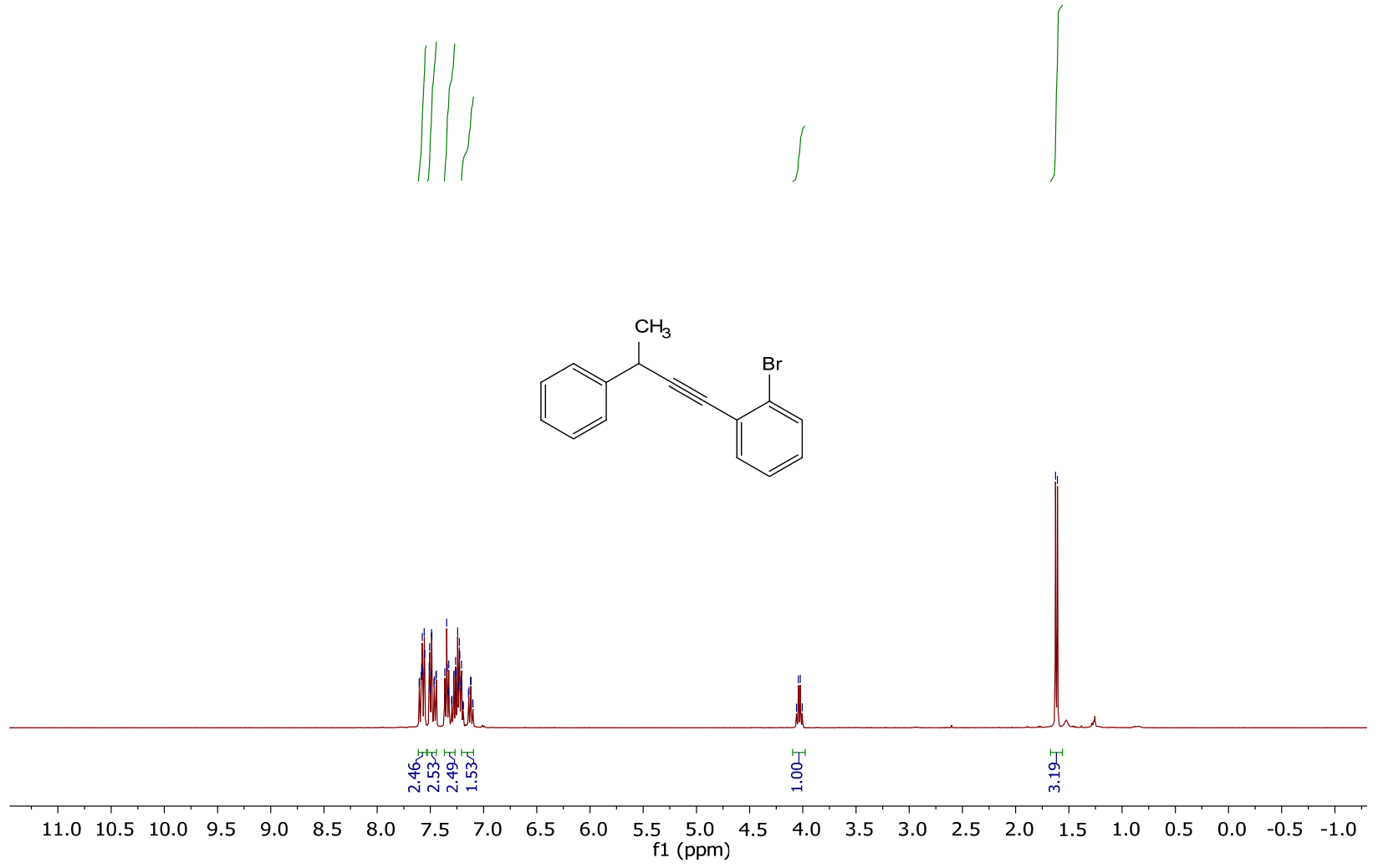


\section{${ }^{13} \mathrm{C}\left\{{ }^{1} \mathrm{H}\right\}$ NMR of $13 \mathrm{~d}\left(\mathrm{CDCl}_{3}, 100 \mathrm{MHz}\right)$}

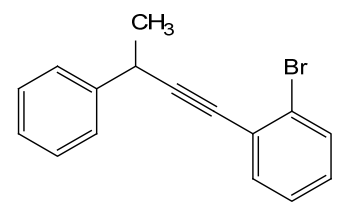

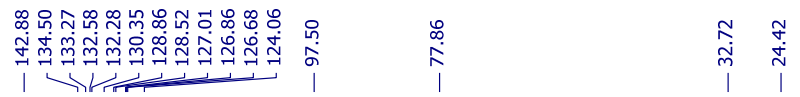

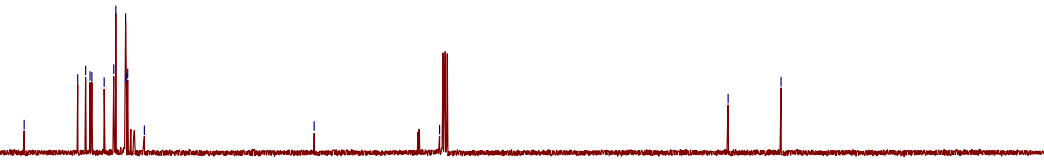

$\begin{array}{llllllllllllllllllllllllllllllllll}230 & 220 & 210 & 200 & 190 & 180 & 170 & 160 & 150 & 140 & 130 & 120 & 110 & 100 & 90 & 80 & 70 & 60 & 50 & 40 & 30 & 20 & 10 & 0 & -10\end{array}$ 
${ }^{1} \mathrm{H}$ NMR of $13 \mathrm{e}\left(\mathrm{CDCl}_{3}, 400 \mathrm{MHz}\right)$

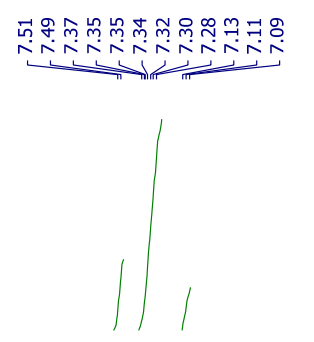

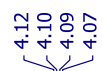

প্ণ
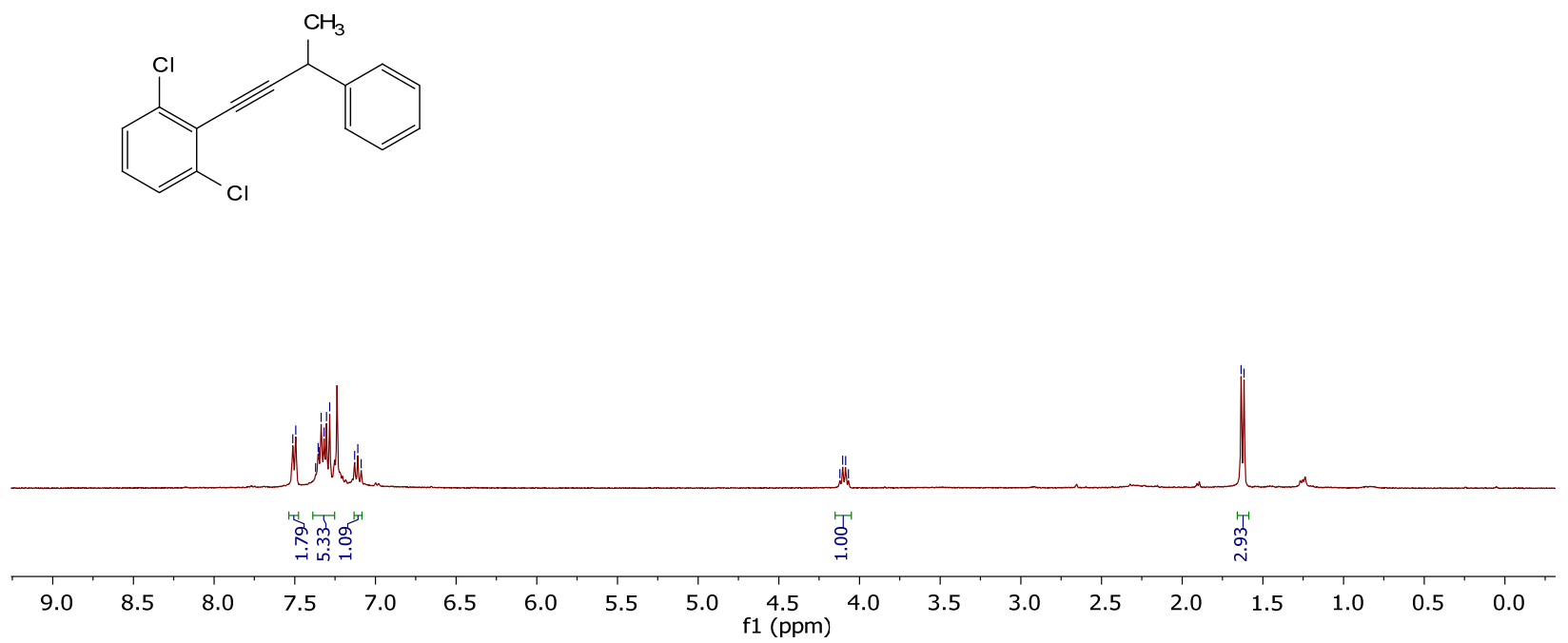
${ }^{13} \mathrm{C}\left\{{ }^{1} \mathrm{H}\right\}$ NMR of $13 \mathrm{e}\left(\mathrm{CDCl}_{3}, 100 \mathrm{MHz}\right)$

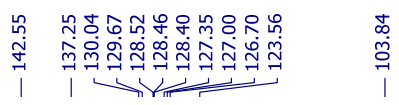

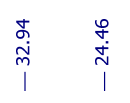
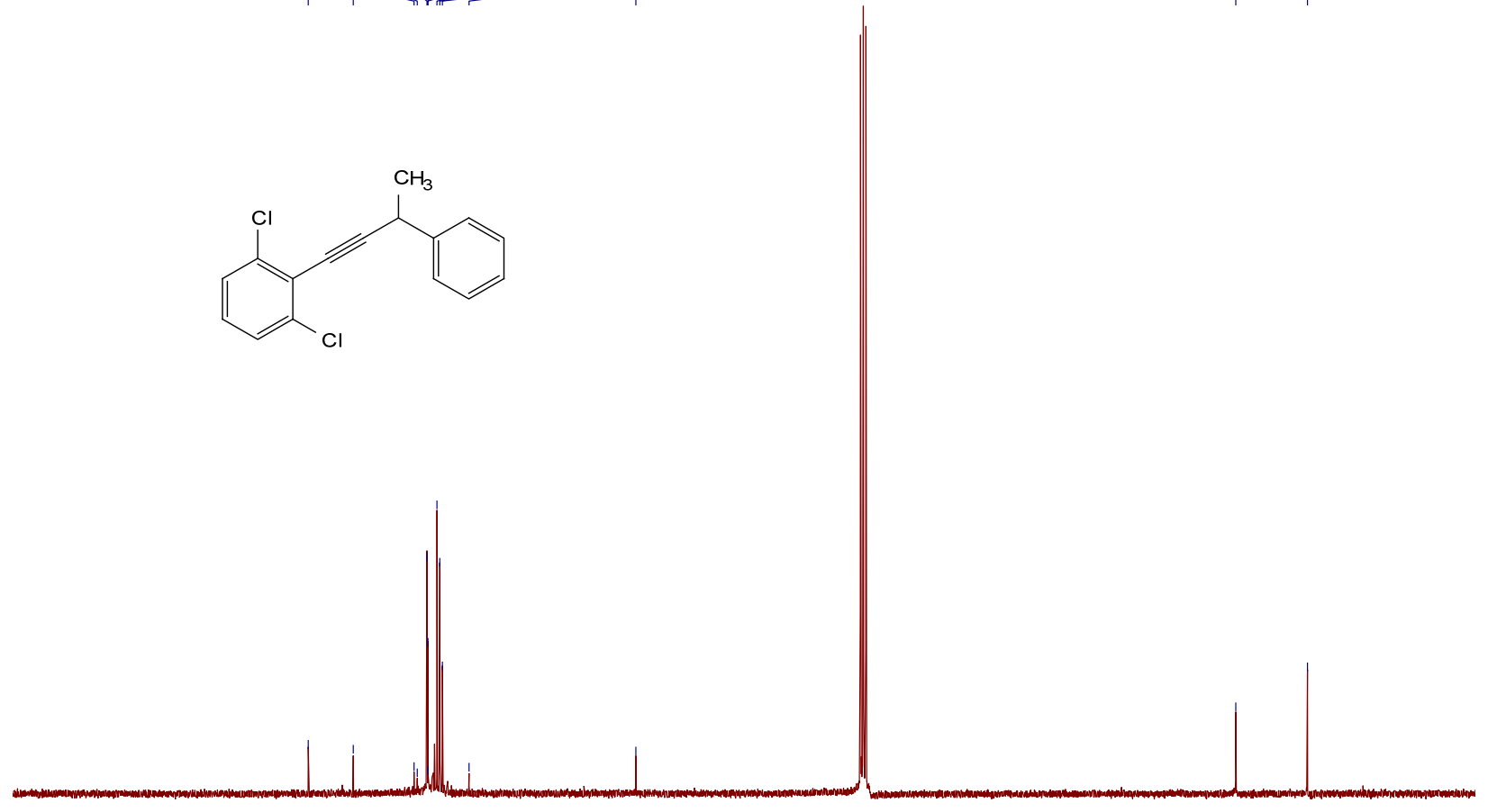
${ }^{1} \mathrm{H}$ NMR of $13 f\left(\mathrm{CDCl}_{3}, 400 \mathrm{MHz}\right)$
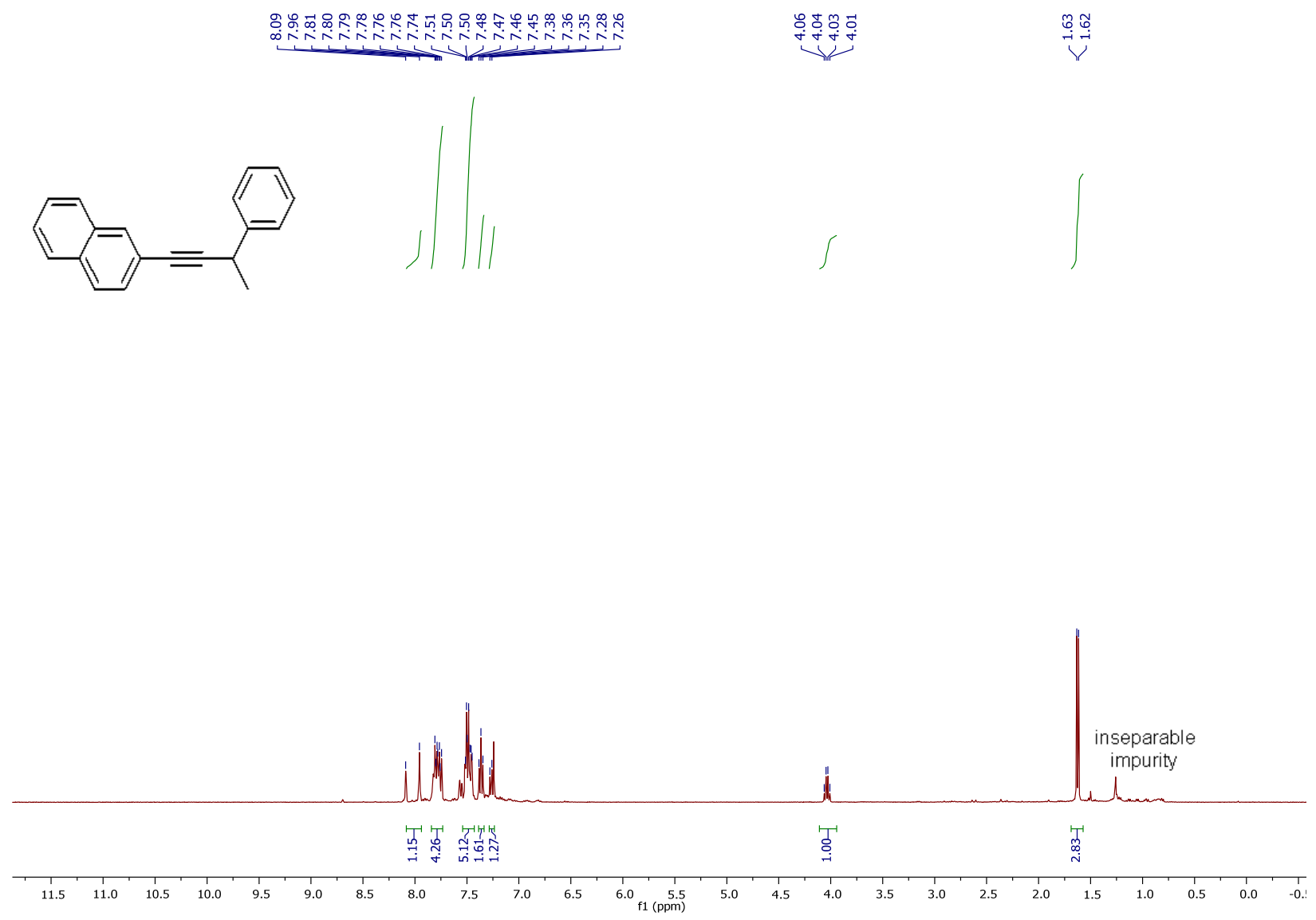


\section{${ }^{13} \mathrm{C}\left\{{ }^{1} \mathrm{H}\right\}$ NMR of $13 \mathrm{f}\left(\mathrm{CDCl}_{3}, 100 \mathrm{MHz}\right)$}

શิ ஜํำ

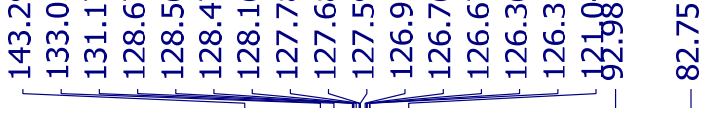

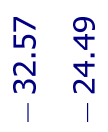<smiles>CC(C#Cc1ccc2ccccc2c1)c1ccccc1</smiles>
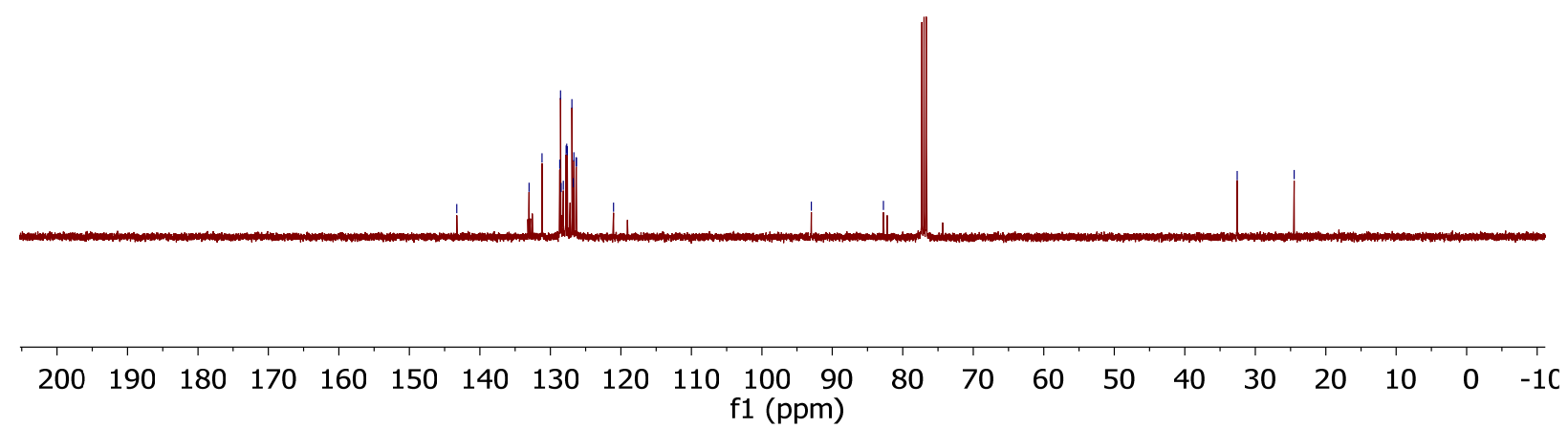
${ }^{1} \mathrm{H}$ NMR of $13 \mathrm{~g}\left(\mathrm{CDCl}_{3}, 400 \mathrm{MHz}\right)$
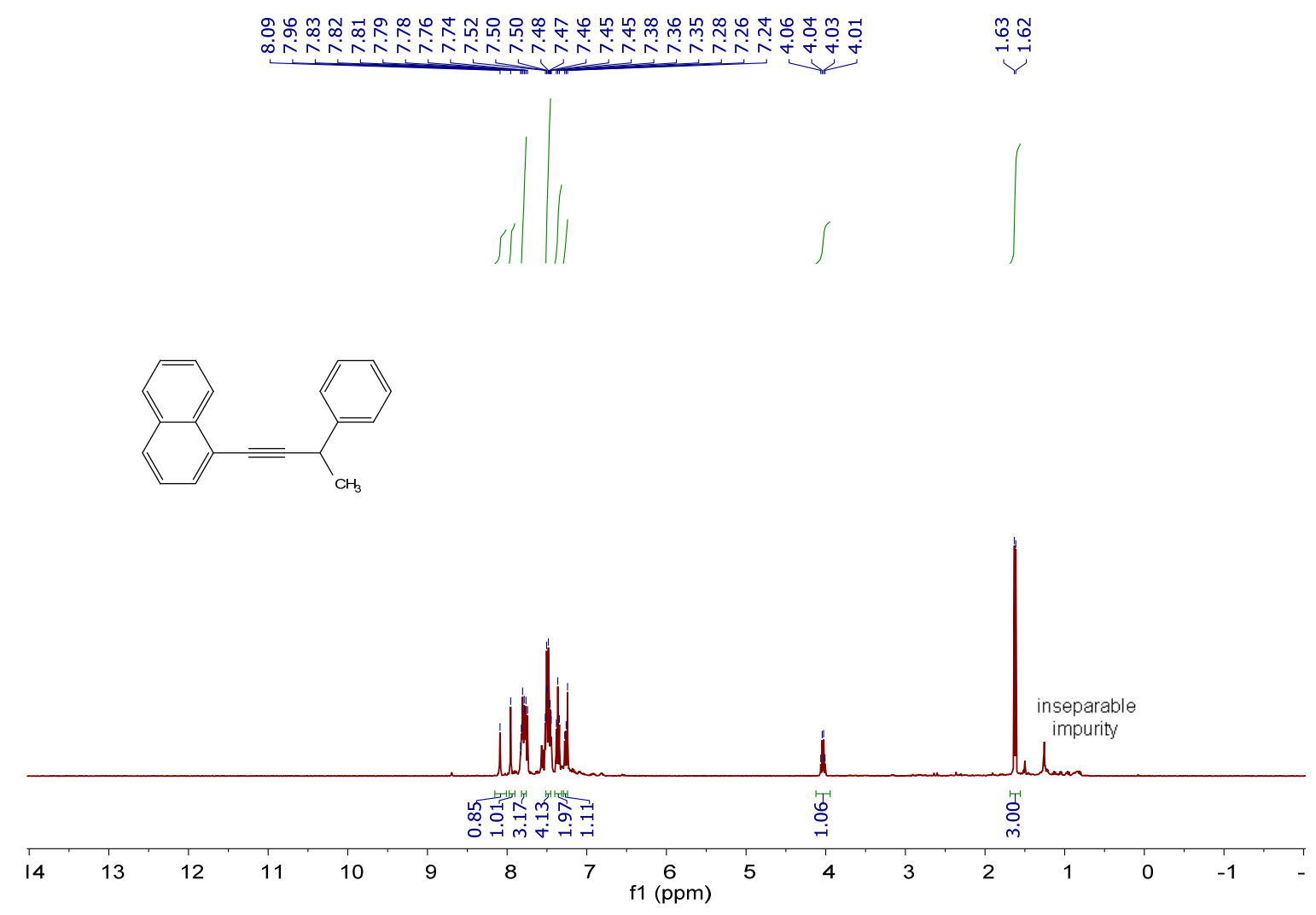
${ }^{13} \mathrm{C}\left\{{ }^{1} \mathrm{H}\right\}$ NMR of $13 \mathrm{~g}\left(\mathrm{CDCl}_{3}, 100 \mathrm{MHz}\right)$
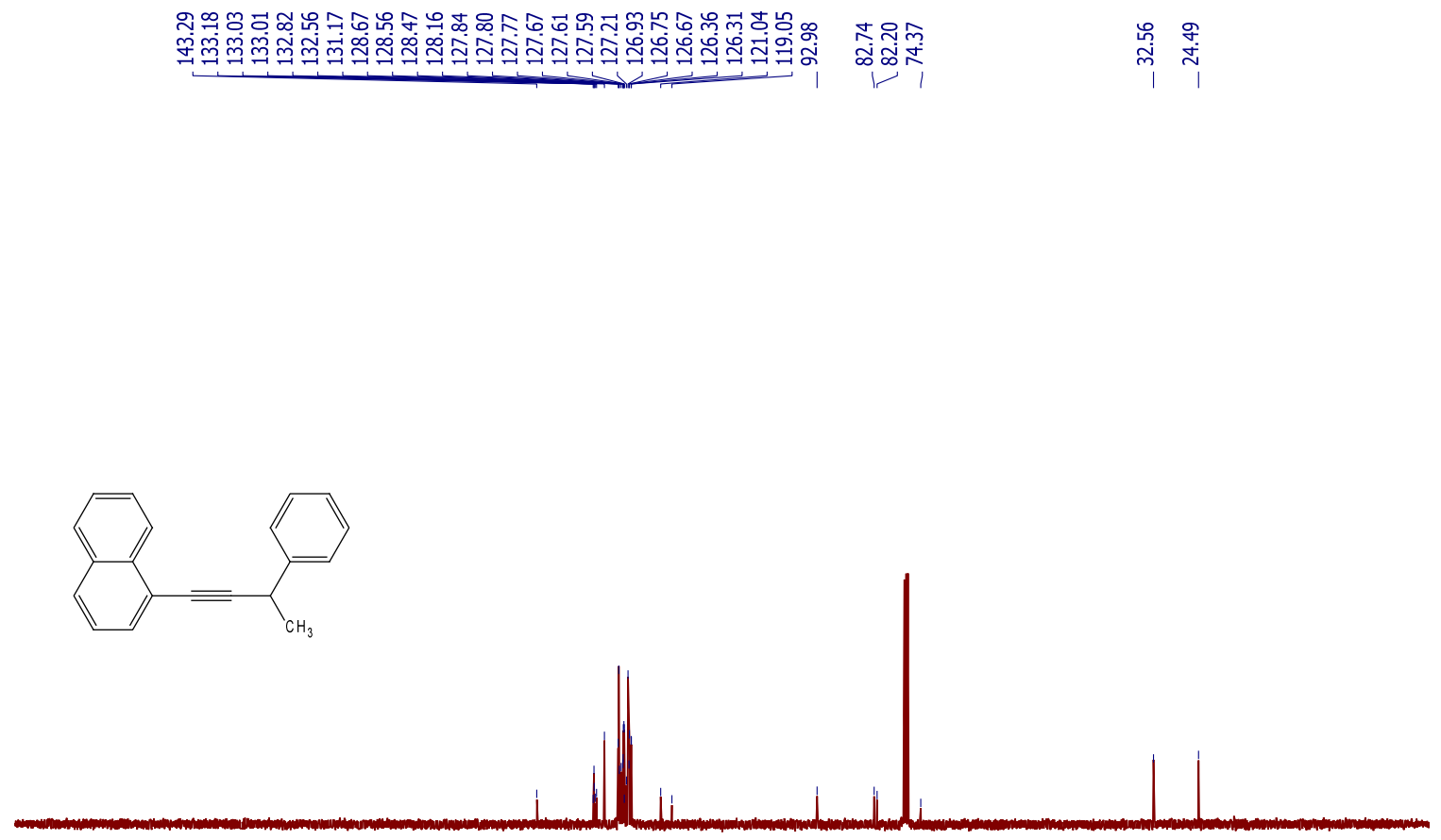

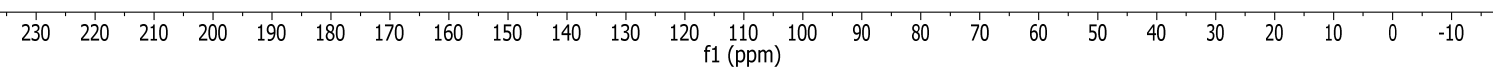


${ }^{1} \mathrm{H}$ NMR of $13 \mathrm{~h}\left(\mathrm{CDCl}_{3}, 400 \mathrm{MHz}\right)$

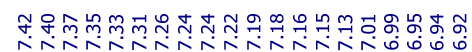

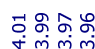

ำ
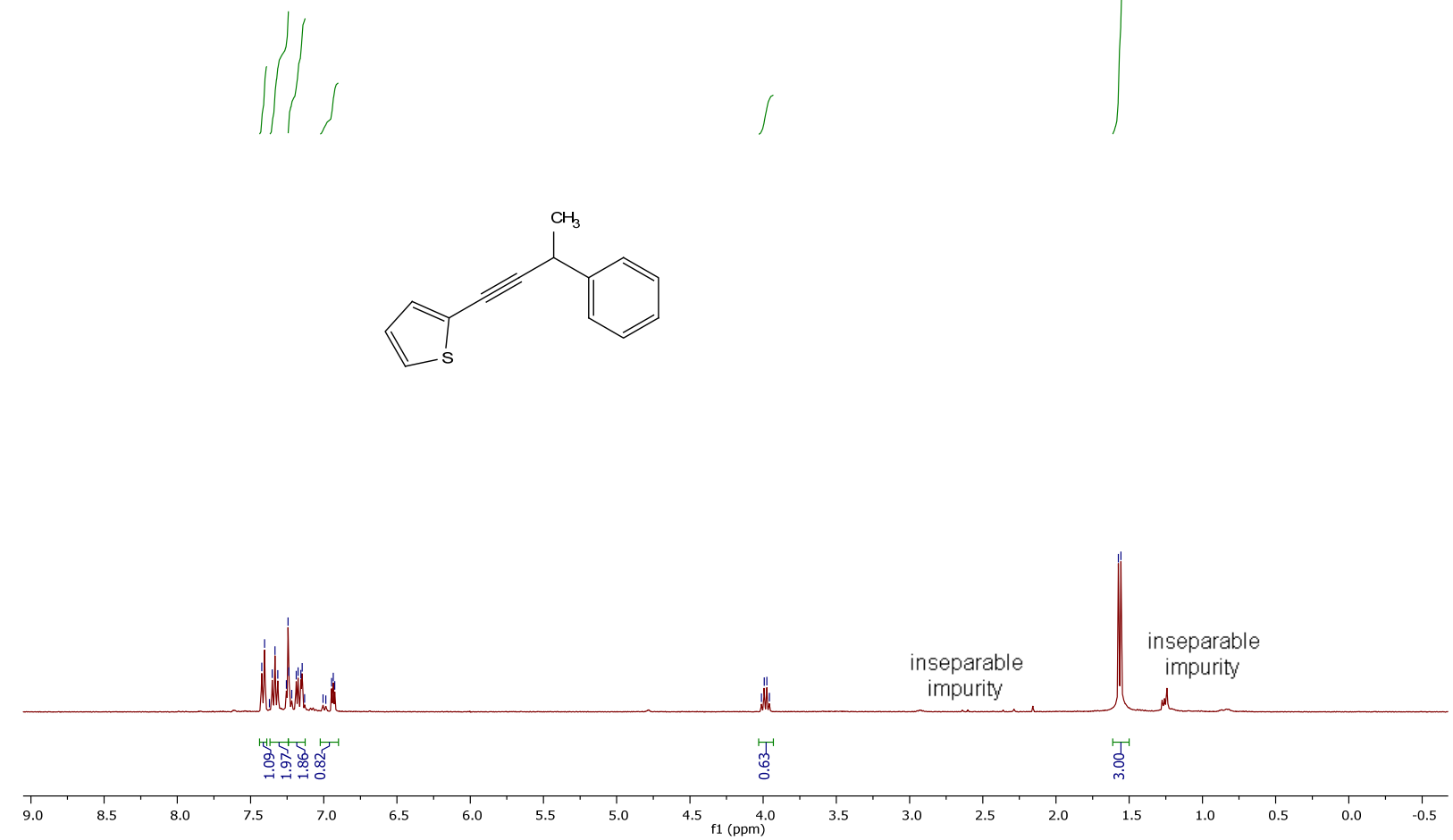

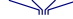

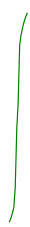


${ }^{13} \mathrm{C}\left\{{ }^{1} \mathrm{H}\right\}$ NMR of $13 \mathrm{~h}\left(\mathrm{CDCl}_{3}, 100 \mathrm{MHz}\right)$

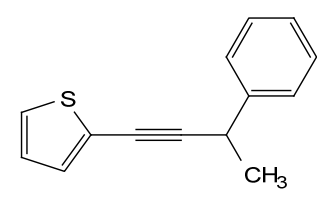

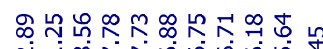

守

守

$\stackrel{\substack{i \\ i}}{\stackrel{N}{\sim}}$

$\mathrm{CH}_{3}$

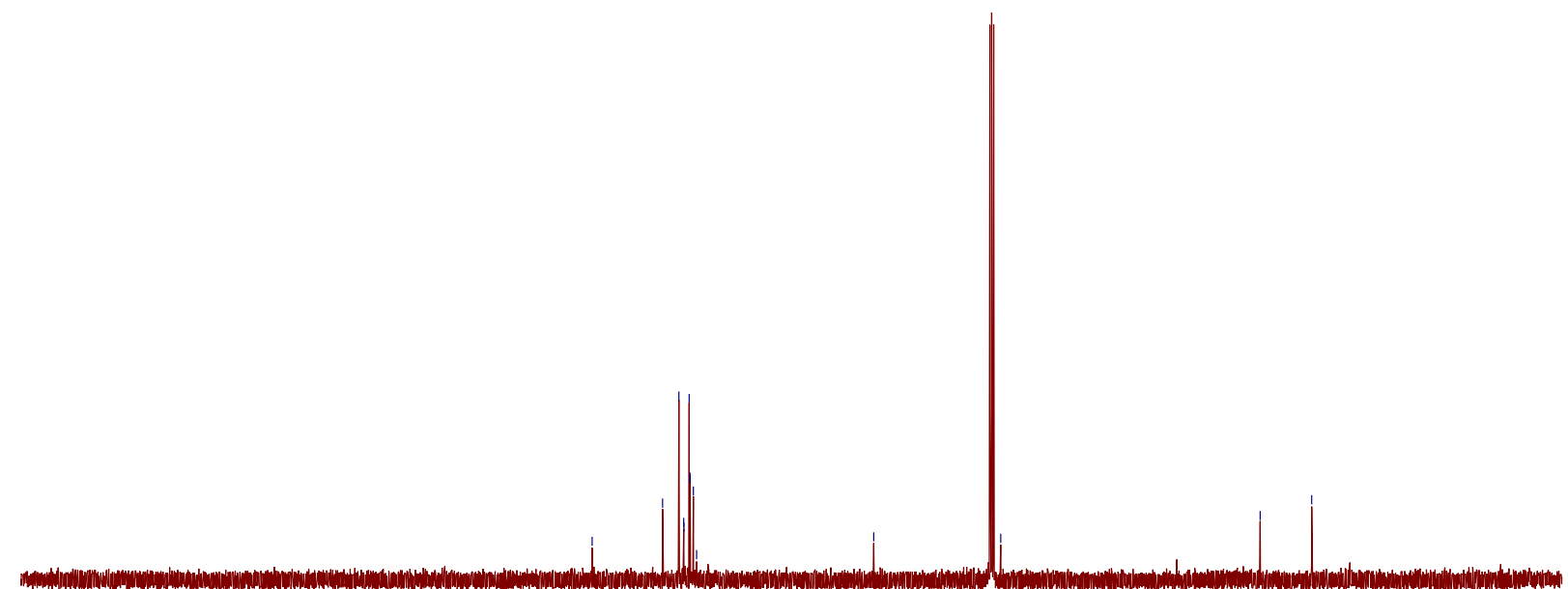

$\begin{array}{llllllllllllllllllllllllll}230 & 220 & 210 & 200 & 190 & 180 & 170 & 160 & 150 & 140 & 130 & 120 & 110 & 100 & 90 & 80 & 70 & 60 & 50 & 40 & 30 & 20 & 10 & 0 & -10\end{array}$ 
${ }^{1} \mathrm{H}$ NMR of 13i (CDCl $\left.3,400 \mathrm{MHz}\right)$

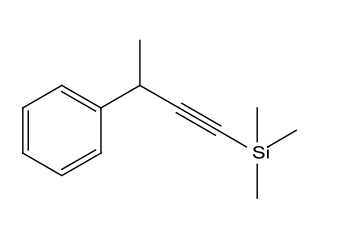

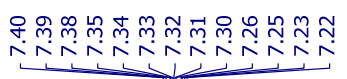

مim

mim

ํำ

$\stackrel{9}{\circ}$
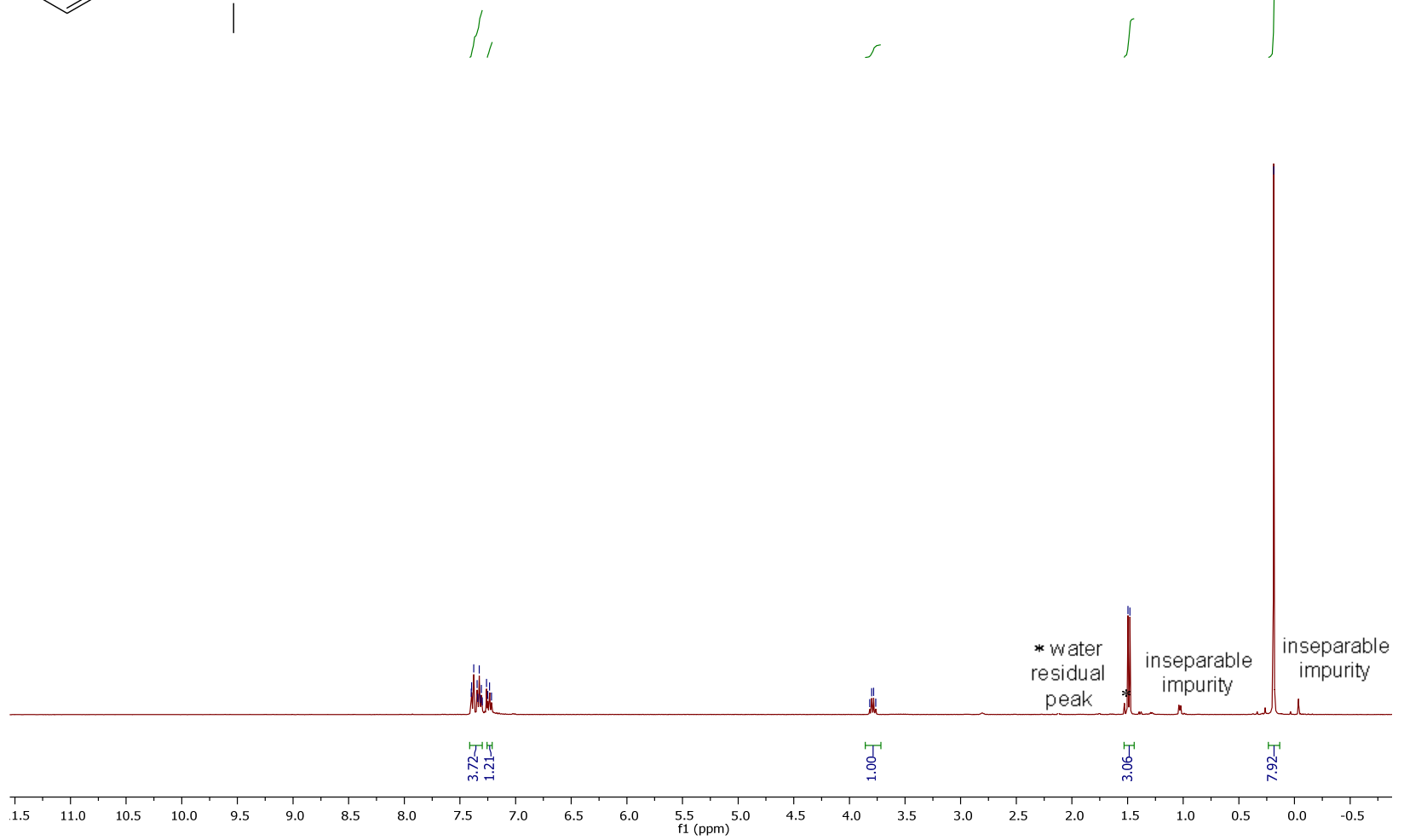
${ }^{13} \mathrm{C}\left\{{ }^{1} \mathrm{H}\right\}$ NMR of $13 \mathrm{i}\left(\mathrm{CDCl}_{3}, 100 \mathrm{MHz}\right)$<smiles>CC(C#C[Si](C)(C)C)c1ccccc1</smiles>

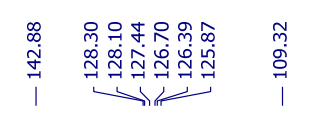

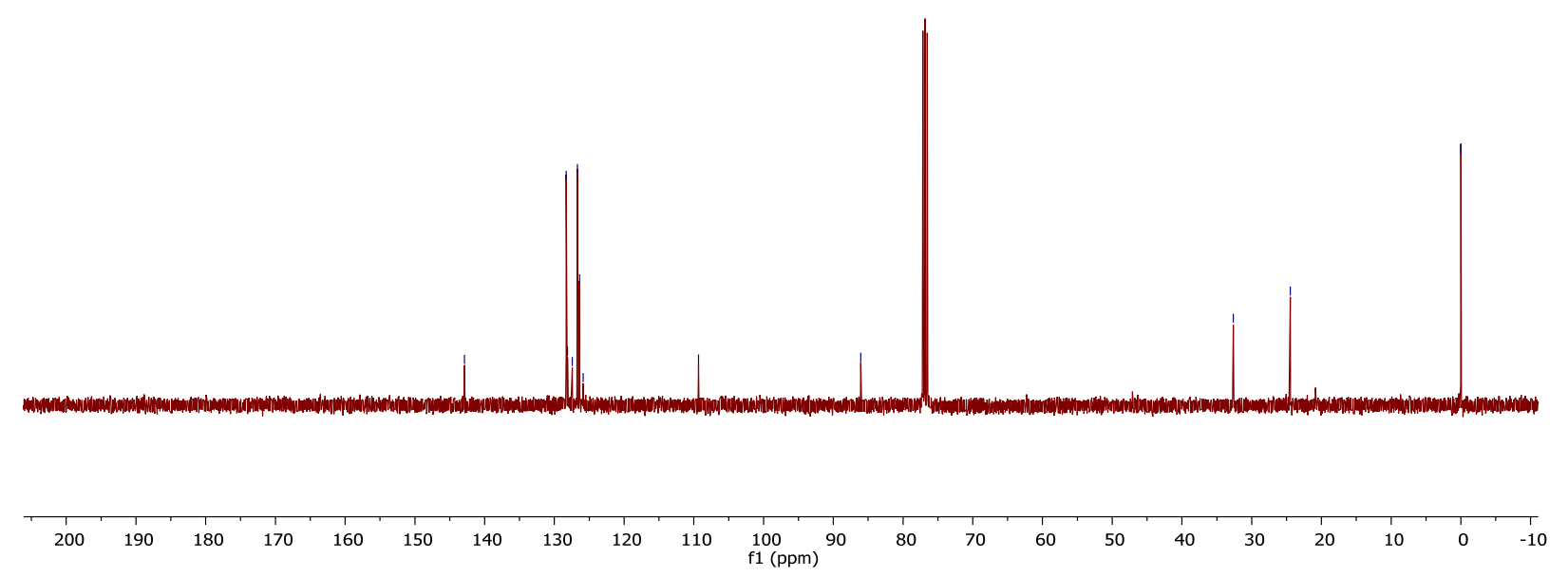


${ }^{1} \mathrm{H}$ NMR of $13 \mathrm{j}\left(\mathrm{CDCl}_{3}, 400 \mathrm{MHz}\right)$

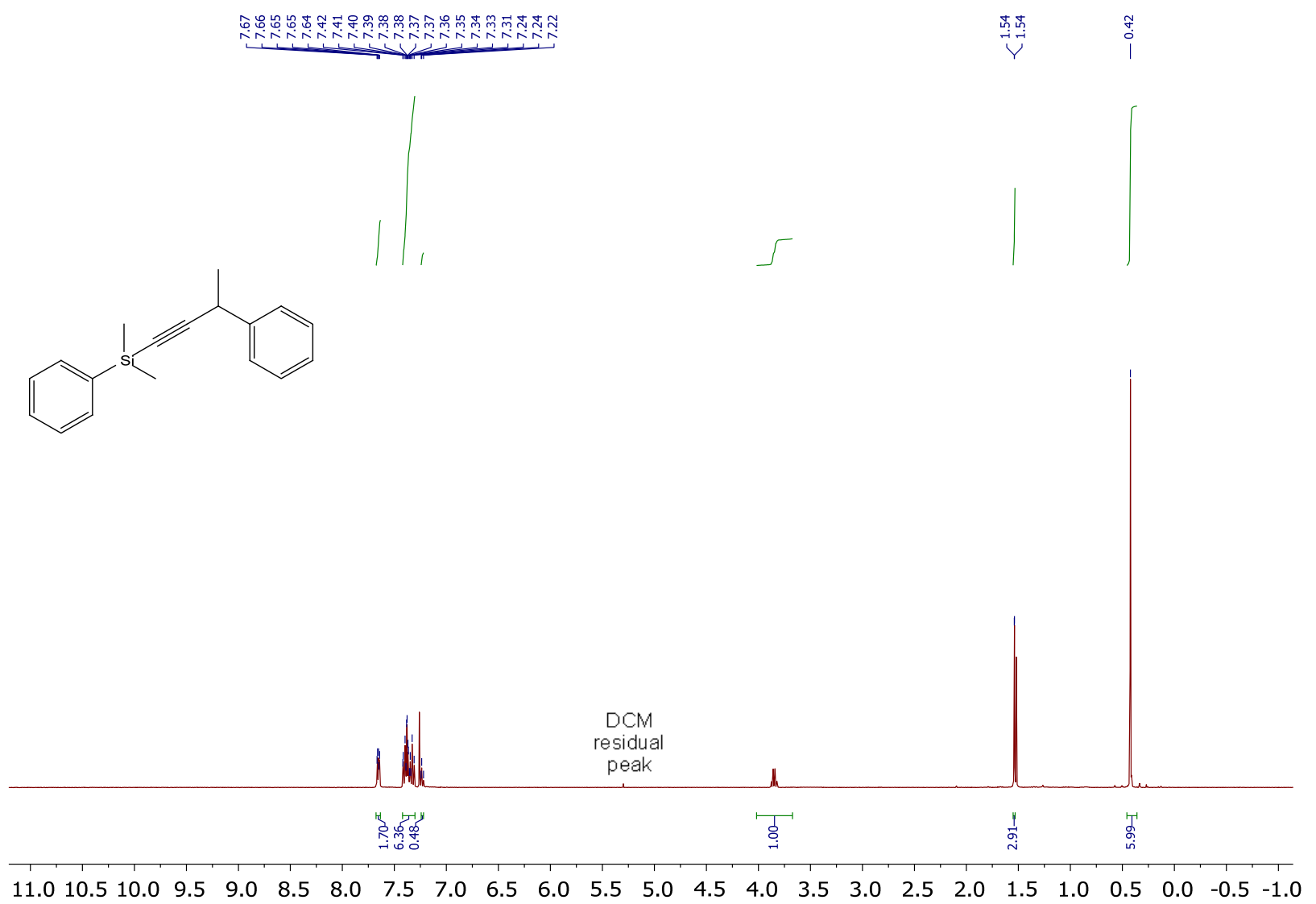


${ }^{13} \mathrm{C}\left\{{ }^{1} \mathrm{H}\right\}$ NMR of $13 \mathrm{j}\left(\mathrm{CDCl}_{3}, 100 \mathrm{MHz}\right)$

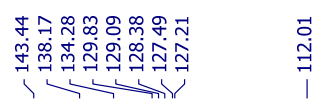
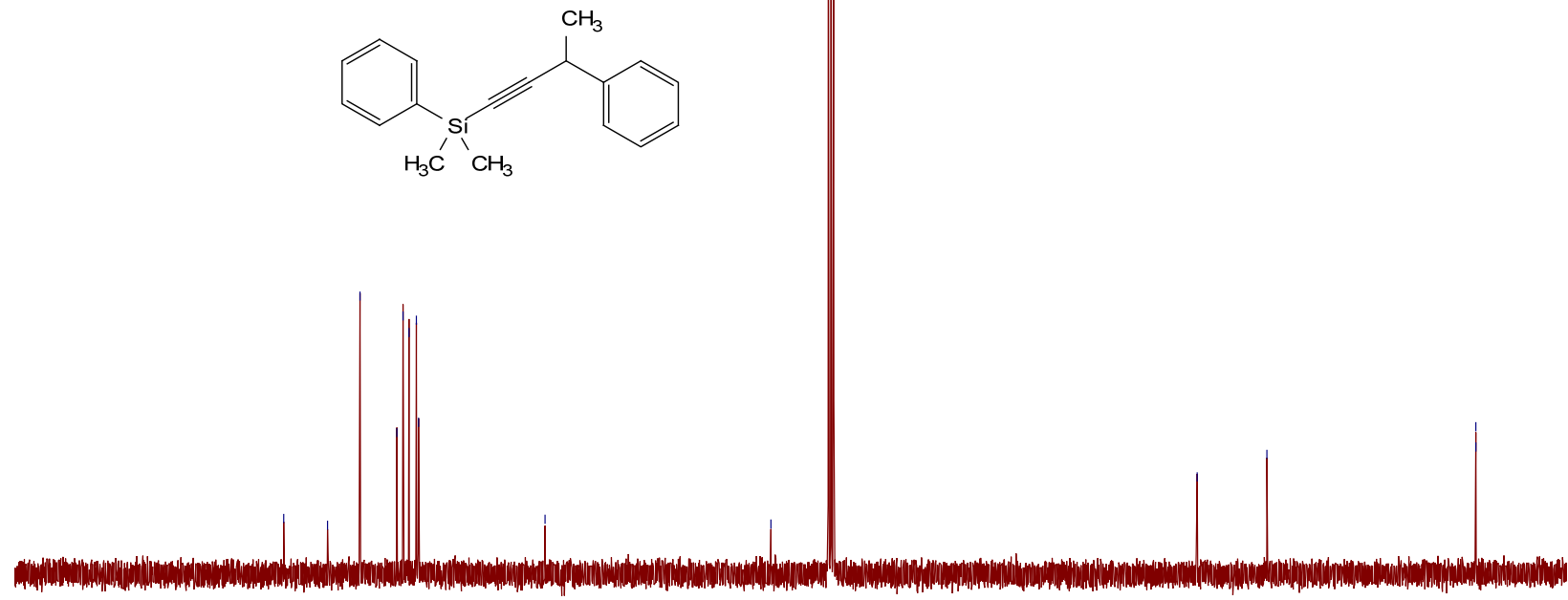

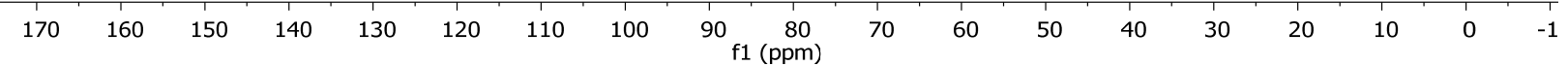




\section{${ }^{1} \mathrm{H}$ NMR of 13k $\left(\mathrm{CDCl}_{3}, 400 \mathrm{MHz}\right)$}

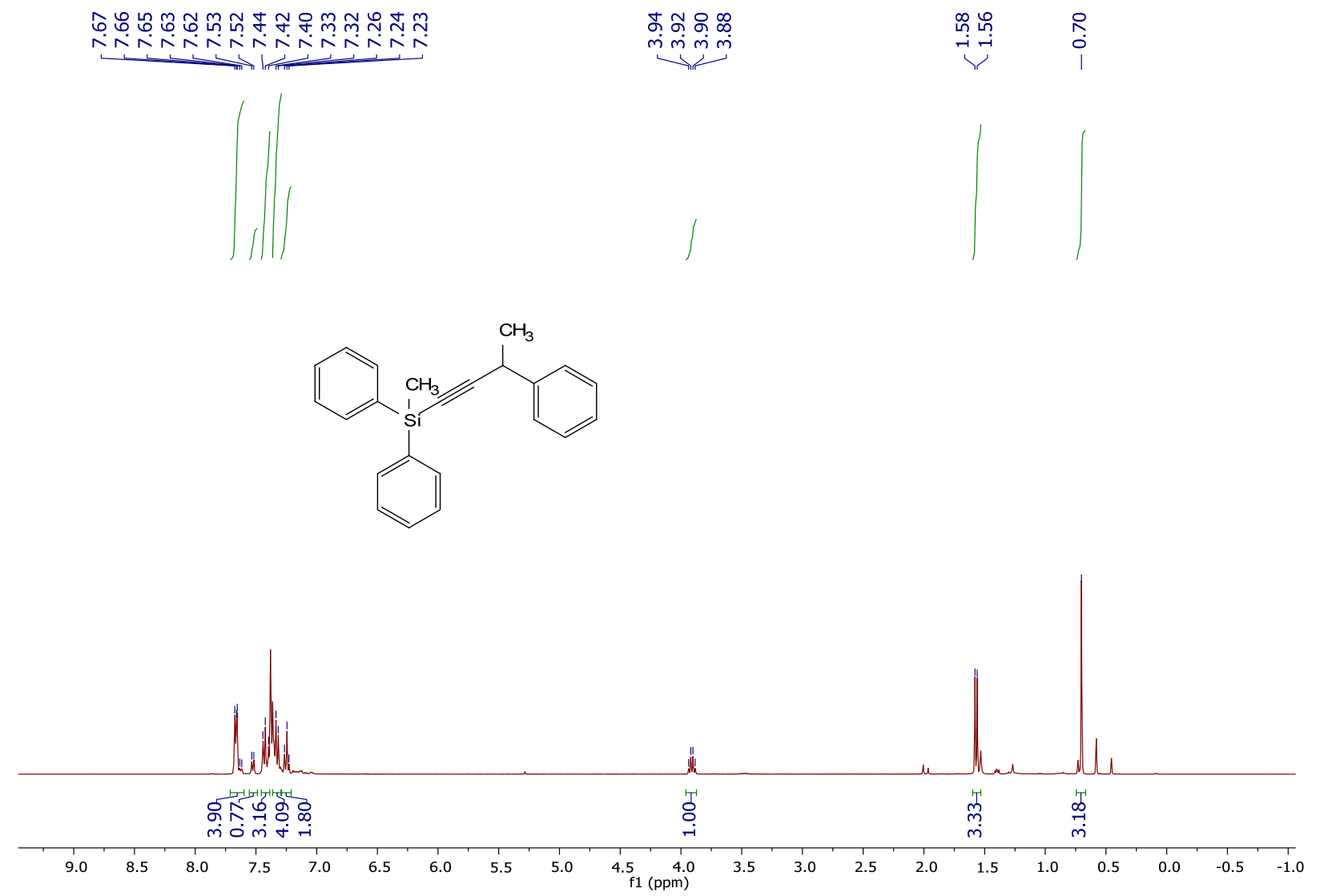




\section{${ }^{13} \mathrm{C}\left\{{ }^{1} \mathrm{H}\right\}$ NMR of $13 \mathrm{k}\left(\mathrm{CDCl}_{3}, 100 \mathrm{MHz}\right)$}

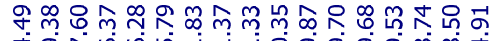

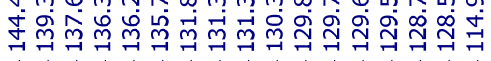

年<smiles>CC(C#C[Si](C)(c1ccccc1)c1ccccc1)c1ccccc1</smiles>

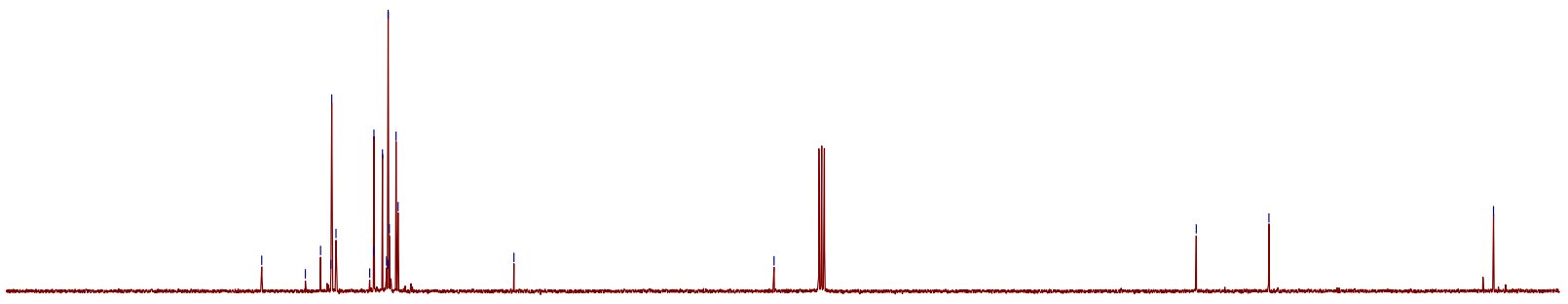

